Università degli Studi di Udine

Dipartimento di Fisica

Dottorato di Ricerca in Matematica e Fisica

Ciclo XVIII

\title{
Search for \\ Chargino and Neutralino production in the trilepton channel with the CDF Run II detector
}

Coordinatore: Prof. Pietro Corvaja

Supervisore: Prof. Giovanni Pauletta

Dottoranda: Melisa Rossi 
à mon grandpère,

à toute ma famille. 
“... non, rien de rien, non, je ne regrette rien ..." Edith Piaf. 



\section{Contents}

$\begin{array}{ll}\text { Introduction } & 7\end{array}$

1 Beyond the Standard Model $\quad 3$

1.1 The Standard Model . . . . . . . . . . . . . . . . . . 3

1.2 Motivation to go beyond the Standard Model . . . . . . . . . . . . 4

1.3 Supersymmetry . . . . . . . . . . . . . . . . 5 5

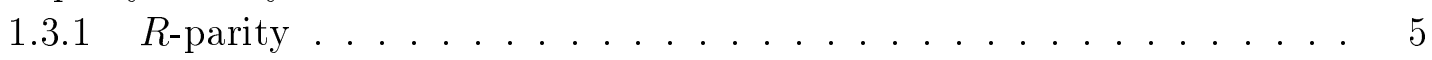

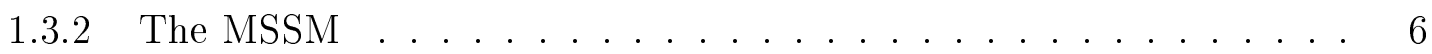

1.3.3 Constraints on SUSY models . . . . . . . . . . . . . 6

1.3.4 Neutralinos and Charginos .............. 7

2 Tevatron and CDF for Run II $\quad 19$

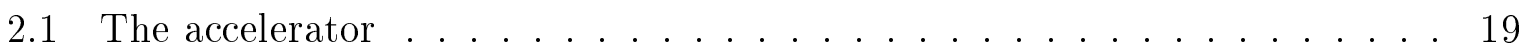

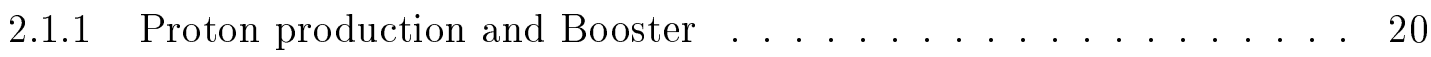

2.1 .2 The Main Injector . . . . . . . . . . . . . . . 22

2.1.3 Antiproton production and cooling . . . . . . . . . . . 22

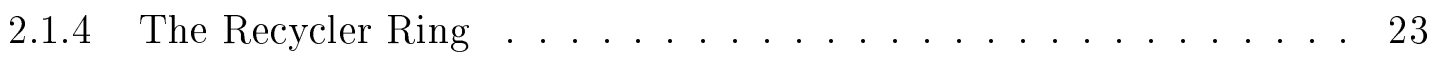

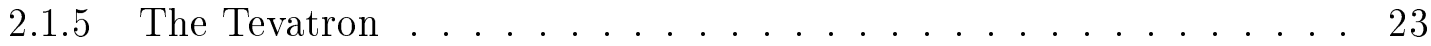

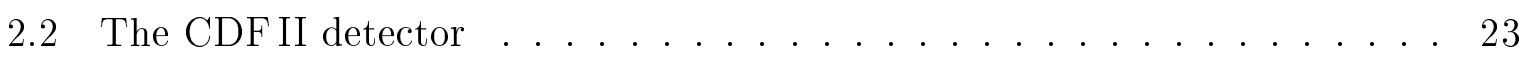

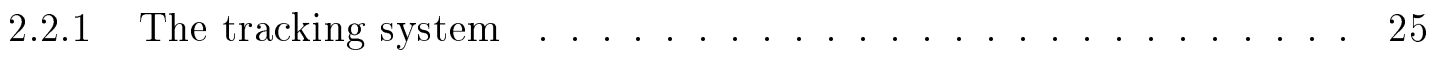

2.2 .2 The time of flight . . . . . . . . . . . . . . . 28

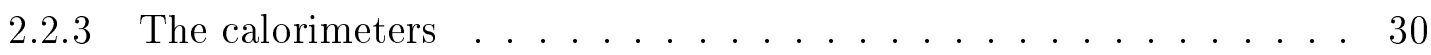

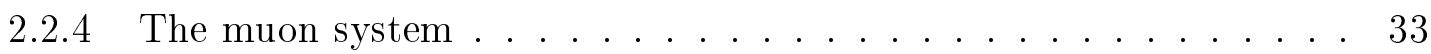

2.2.5 The Cherenkov luminosity counters . . . . . . . . . . . . 35

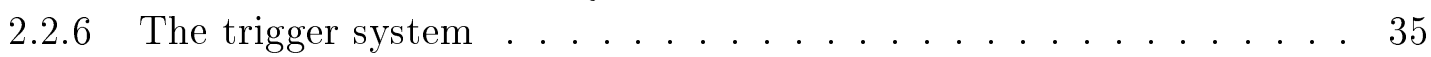

3 The CDF trigger $\quad 39$

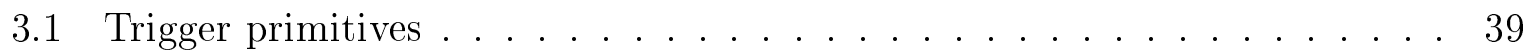

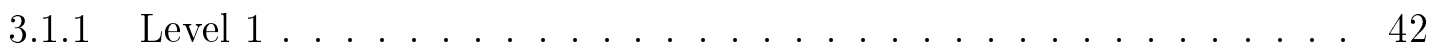

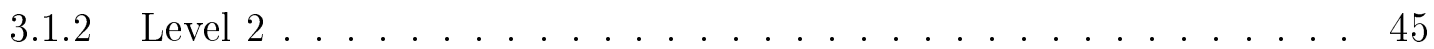

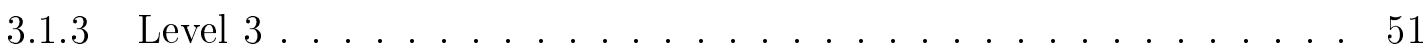

3.2 The SUSY DILEPTON triggers . . . . . . . . . . . . . . 51

3.2.1 The SUSY DILEPTON trigger strategy . . . . . . . . . . 52

4 Event reconstruction $\quad \mathbf{5 7}$

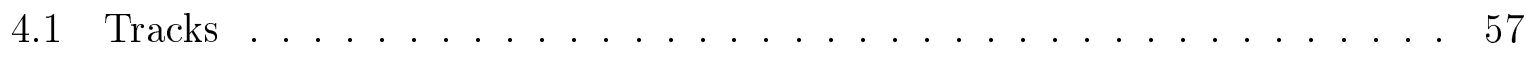

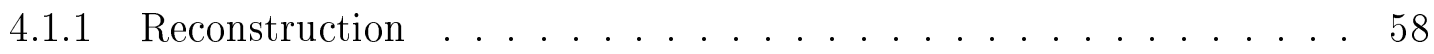


4.1 .2 Resolutions . . . . . . . . . . . . . . . . . . . . . 59

4.2 Primary vertex reconstruction . . . . . . . . . . . . . . 59

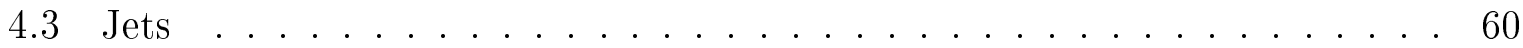

4.3 .1 Jet Clustering . . . . . . . . . . . . . . . . . . . . 61

4.3 .2 Jet corrections . . . . . . . . . . . . . . . . . . . . 62

4.4 Leptons . . . . . . . . . . . . . . . . . . . . . . 64

4.4 .1 Electrons . . . . . . . . . . . . . . . . 65

4.4 .2 Muons . . . . . . . . . . . . . . . . . 69

4.5 Missing energy . . . . . . . . . . . . . . . . 70

5 Analysis signature and data samples $\quad 75$

5.1 The analysis signature . . . . . . . . . . . . . . 75

5.2 A blind analysis . . . . . . . . . . . . . . 77

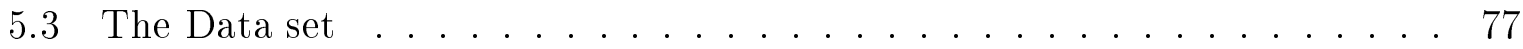

5.3 .1 Luminosity . . . . . . . . . . . . . . . 78

5.4 Monte Carlo samples . . . . . . . . . . . . . . . . . . 78

5.4 .1 The signal sample . . . . . . . . . . . . . . . 78

5.4 .2 Backgrounds . . . . . . . . . . . . . . . . . . 79

5.5 Fake lepton contribution . . . . . . . . . . . . . . 82

6 The Data analysis $\quad 87$

6.1 Data and $\mathrm{MC}$ comparison $\ldots \ldots \ldots \ldots \ldots$. . . . . . . 87

6.2 Event selection . . . . . . . . . . . . . . . . 88

6.3 The dielectron and dimuon events . . . . . . . . . . . . . 90

6.4 The $e \mu$ channel: effect of the analysis cuts . . . . . . . . . . . . . . 91

6.5 Control regions for $e \mu$ events . . . . . . . . . . . . . . . . . 98

6.5.1 Dilepton control regions . . . . . . . . . . . . . . 99

6.5.2 Trilepton control regions . . . . . . . . . . . . . . . . 112

6.6 Summary and discussion . . . . . . . . . . . . . . . . . 112

6.7 Future plans . . . . . . . . . . . . . . . . . . . . . 113

$\begin{array}{ll}\text { Conclusions } & 117\end{array}$

A SUSY DILEPTON Trigger Monitoring 119

A.1 Motivation . . . . . . . . . . . . . . . . . . . . . . . . . . . 119

A.2 Monitoring Variable Definition . . . . . . . . . . . . . . . . 120

A.3 Suspect Run Definition . . . . . . . . . . . . . . . . . . . . . . . . 127

A.4 Good Run list on the basis of R . . . . . . . . . . . . . 127

A.5 Good run list on the basis of cross section stability . . . . . . . . 130

A.6 Conclusions . . . . . . . . . . . . . . . . . . . . . 130

B Electron Trigger Efficiencies $\quad 139$

B.1 Introduction . . . . . . . . . . . . . . . . . . . . . . . 139

B.2 Sample . . . . . . . . . . . . . . . . . . . . . . . . 140

B.3 Efficiency Calculation and Fits . . . . . . . . . . . . . . . 141

B.4 Matching Offline Electrons to Online Objects . . . . . . . . . . . . 146

B.4.1 Matching Offline EM Cluster to L1 Trigger Tower . . . . . . . . . 146

B.4.2 Matching Offline Track to XFT Track . . . . . . . . . . . 146 
B.4.3 Matching Offline EM Cluster to L2 Cluster . . . . . . . . . . . . . 146

B.5 Efficiency of Plug Electrons . . . . . . . . . . . . . . . 147

B.5.1 L1_EM8 EfFICIENCY . . . . . . . . . . . . . . 147

B.5.2 L2_PEM8 EfFICIENCY . . . . . . . . . . . 150

B.6 Efficiency of Central Electrons . . . . . . . . . . . . . . . . 151

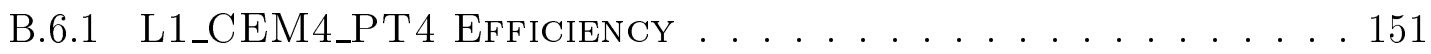

B.6.2 L1_CEM8_PT8 EffiCIENCY . . . . . . . . . . . 153

B.6.3 2-Dimensional Fit for L1 Central Electron Primitives . . . . . . . 155

B.6.4 L2_CEM4_PT4_CES3 EFFICIENCY . . . . . . . . . . . . 156

B.6.5 L2_CEM8_PT8_CES3 EFFICIENCY . . . . . . . . . . . 158

B.7 Systematics . . . . . . . . . . . . . . . . . 158

$\begin{array}{ll}\text { List of figures } & 165\end{array}$

$\begin{array}{lr}\text { List of tables } & 167\end{array}$ 



\section{Introduction}

The Standard Model provides a successful description of presently known phenomena. However, some important questions still remain unanswered and it is certain that at the Plank scale, where quantum gravitational effects become relevant, a new framework will be needed. Supersymmetry is one of the most appealing extensions of the Standard Model and it could naturally resolve many issues.

This thesis is part of a search for supersymmetry being conduced by the CDF Collaboration at the TEVATRON collider. The work focuses on the search for the superpartners of gauge and Higgs bosons. More specifically, the search is concentrated on the associated production of chargino and neutralino decaying into leptons. Multilepton final states are particularly clean signatures when dealing with the abundance of QCD events produced at hadron colliders. The expected signal has a production cross section of about $0.5 \mathrm{pb}$ which has to be extracted from a $p \bar{p}$ cross section of about $50 \mathrm{mb}$. In these circumstances the process signature and the choice of the associated trigger become crucial. This analysis focuses on final states with one electron and one muon plus an additional lepton. The analysis was performed as a blind analysis, in which kinematic regions where the supersymmetric signal is not expected to be significant are investigated first. Only when the ability to describe standard model backgrounds in these regions is proven, is the signal region studied.

In Chapter 1 a brief introduction to supersymmetry is given with particular emphasis on the leptonic decays of chargino and neutralino. The last section of the first chapter is dedicated to the description of prior searches at colliders. In Chapter 2 the TEVATRON collider and the CDF detector are illustrated. Chapter 3 and Chapter 4 are dedicated to a more detailed description of the CDF trigger system and the event recontruction respectively. The approach used in the analysis is outlined in Chapter 5. In this chapter the data and Monte Carlo samples are also described. Chapter 6 focuses on the analysis itself concluding with the description of the current status of the search. 



\section{Chapter 1}

\section{Beyond the Standard Model}

In this chapter a brief introduction of the Standard Model and the open issues that this theory leaves unanswered is presented. Several possible roads for going beyond the Standard Model have been explored by theoretitians. Since this thesis focuses on an experimental search based on the predictions of the supersymmetric extension of the Standard Model, a simple introduction of the supersymmetric theory is also given in this chapter.

\subsection{The Standard Model}

The Standard Model (SM) is a gauge theory ([1][2][3][4]), based on the symmetry group

$$
S U(3)_{C} \otimes S U(2)_{L} \otimes U(1)_{Y}
$$

which describes strong, weak and electromagnetic interactions, via the exchange of the corresponding spin-1 gauge fields: 8 massless gluons and 1 massless photon for the strong and electromagnetic interactions, respectively, and 3 massive bosons $W^{ \pm}$and $Z$ for the weak interaction. The fermionic matter content is given by the known leptons and quarks, which are organized in a 3-fold family structure:

$$
\left[\begin{array}{ll}
\nu_{e} & u \\
e^{-} & d
\end{array}\right] \quad, \quad\left[\begin{array}{ll}
\nu_{\mu} & c \\
\mu^{-} & s
\end{array}\right] \quad, \quad\left[\begin{array}{cc}
\nu_{\tau} & t \\
\tau^{-} & b
\end{array}\right]
$$

where (each quark appears in 3 different "colours")

$$
\left[\begin{array}{cc}
\nu_{l} & q_{u} \\
l^{-} & q_{d}
\end{array}\right] \equiv\left(\begin{array}{c}
\nu_{l} \\
l^{-}
\end{array}\right)_{L},\left(\begin{array}{c}
q_{u} \\
q_{d}
\end{array}\right)_{L}, l_{R}^{-}, q_{u R}, q_{d R}
$$

plus the corresponding antiparticles. Thus, the left-handed fields are $S U(2)_{L}$ doublets, while their right-handed partners transform as $S U(2)_{L}$ singlets. The three fermionic families in Eq. 1.2 appear to have identical properties (gauge interactions); they only differ by their mass and their flavour quantum number.

The gauge symmetry is broken by the vacuum which triggers the Spontaneous Symmetry Breaking (SSB) of the electroweak group to the electromagnetic subgroup: 


$$
S U(3)_{C} \otimes S U(2)_{L} \otimes U(1)_{Y} \quad \rightarrow \quad S U(3)_{C} \otimes U(1)_{Q E D}
$$

The SSB mechanism generates the masses of the weak gauge bosons, and gives rise to the appearence of a physical scalar particle in the model, the Higgs boson. The fermion masses and mixings are also generated through the SSB.

The Standard Model is a remarkably successful theory from the phenomenological point of view. It provides a very elegant theoretical framework able to describe the known experimental facts (with the possible caveat of the Higgs boson discovery) with high precision.

\subsection{Motivation to go beyond the Standard Model}

Albeit its many successes, the SM has been regarded as only a low-energy effective theory ${ }^{1}$ of a more fundamental theory underlying the SM itself. In fact, there are a number of theoretical and phenomenological issues that the SM fails to address adequately:

- Hierarchy problem. Phenomenologically the mass of the Higgs boson associated with the electroweak symmetry breaking must be in the electroweak range. However, radiative corrections to the Higgs mass are quadratically dependent on the UV cutoff $\Lambda^{2}$, since the masses of fundamental scalar fields are not protected by chiral or gauge symmetries. The "natural" value of the Higgs mass is therefore of $O(\Lambda)$ rather than $O(\sim 100 \mathrm{GeV})$, leading to a destabilization of the hierarchy of the mass scales in the SM.

- Electroweak symmetry breaking (EWSB). In the SM, EWSB is parameterized by the Higgs boson $h$ and its potential $V(h)$. However, the Higgs sector is not constrained by any symmetry principles and it must be put into the theory by hand.

- Gauge coupling unification. The idea that the gauge couplings undergo renormalization group evolution in such a way that they meet at a point at a high energy scale lends credence to the picture of grand unified theories (GUTs). However, precise measurements of the low energy values of the gauge couplings demonstrated that the SM can not describe gauge coupling unification.

- Family structure and fermion masses. The SM does not explain the existence of three families and can only parameterize the strongly hierarchiral values of the fermion masses. Massive neutrinos would imply that the theory should be extended, as in the SM the neutrinos are strictly left-handed and massless, and recent evidence for neutrino oscillations suggest the mass of the third-generation neutrino $\nu_{\tau}$ of about $0.05 \mathrm{eV}$.

\footnotetext{
${ }^{1}$ A theory whose validity is restricted to a certain energy scale.

${ }^{2}$ The higgs mass receives quadratically divergent contributions from its self-energy corrections. For instance, the process where the Higgs doublets splits into a pair of top quarks and come back to the Higgs boson gives a self-energy correction.
} 
- Cosmological challenges. Several difficulties are encountered when trying to build cosmological models based only on the SM particle content. The SM cannot explain baryon asymmetry of the universe; it also does not have a viable candidate for the cold dark matter of the universe. The most serious problem the SM has is its inability to account for the gravitational interactions which are not at all included in the model.

\subsection{Supersymmetry}

Any field theory which aims to play the role of an extension of the SM must first of all reproduce the correct and accurate SM predictions and encompass the observed SM particles and field content as well as its gauge group structure. Moreover new physics contributions and corrections to SM precision measurements must naturally be compatible with experimental results. Supersymmetry (SUSY) is such a theory.

Supersymmetry is a symmetry between bosons and fermions, hence it relates particles with different spins. Since supersymmetry relates the scalar and fermionic sectors, the chiral symmetries which protect the masses of the fermions also protect the masses of the scalars from quadratic divergences, leading to an elegant resolution of the hierarchy problem. The immediate consequence is that quadratic divergences in the Higgs self-energy corrections become logarithmic. All particles in supersymmetric theories fall into supermultiplets, which have both bosonic and fermionic components. In the supersymmetric Lagrangians the fermionic and the bosonic components in the same supermultiplets are always degenerate in mass. This possibility is ruled out by the fact that there is no experimental evidence of SUSY. Therefore supersymmetry must be broken. The mechanism of supersymmerty breaking is not yet understood. In the low-energy effective theories a possible way to induce the SUSY breaking is to add terms to the supersymmetric Lagrangians which break supersymmetry explicitely. In principle, any terms with couplings with positive mass dimensions are candidates. The important constraint is that such explicit supersymmetry-breaking terms should not spoil the motivation discussed earlier, namely to keep the Higgs mass-squared only logarithmically divergent. Such explicit breaking terms of supersymmetry are called "soft" breaking terms. For a detailed theoretical derivation of the supersymmetric Lagrangians (which is beyond the goal of this dissertation) the reader can refer to [5][6][7][8][9].

\subsubsection{R-parity}

In principle the $S U(3)_{C} \otimes S U(2)_{L} \otimes U(1)_{Y}$ gauge invariance allows terms in the superpotential which violate lepton or baryon number. Combinations of such couplings, which would lead to rapid proton decay, must be somehow suppressed. A common assumption is to impose a discrete symmetry called $R$-parity (or matter parity), acting as a multiplicative quantum number, defined as

$$
R_{p}=(-1)^{2 s+3 B+L}
$$


where $\mathrm{s}$ is the spin, $\mathrm{B}$ is the barion quantum number and $\mathrm{L}$ is the leptonic quantum number of the particle. From this definition follows that all SM particles carry even Rparity while all supersymmetric partners are odd due to the $\left(-1^{2 \mathrm{~s}}\right)$ factor. One immediate consequence of a supersymmetric scenario, in which $R$-parity is conserved, is that the lightest particle with odd $R$-parity, the Lightest Supersymmetric Particle (LSP), is stable and superparticles can be produced only pairwise. These two points have quite important implications on the collider phenomenology and cosmology. Since the LSP is stable, its cosmological relic is a good candidate for the Cold Dark Matter particles. In this case, LSP can not be electrically charged and/or strongly interacting; otherwise it should have been detected already. On the other hand, the superparticles can be produced only in pairs and they decay eventually in LSP, which escapes detection. This is why the typical signature of supersymmetry at collider experiments is the missing energy/momentum.

In this dissertation $R$-parity conserving models will always be considered.

\subsubsection{The MSSM}

Once the supersymmetry is explicitely broken while retaining the absence of power divergences, it is possible to promote the SM to a supersymmetric theory. The Minimal Supersymmetric Standard Model (MSSM) is a supersymmetric version of the Standard Model with the minimal particle content as well as with the most general set of soft susy breaking terms. The field content of the MSSM consists of the superfields associated with each field of the SM, plus an additional Higgs doublet.

All scalar superpartners of quarks and leptons are named in the same way with an "s" at the beginning (squarks and sleptons). The superpartners of the Higgs doublets are called "higgsinos". In general, fermionic superpartner fields are named after their bosonic counterpart plus a suffix "ino". Hence spin1/2 superpartners of the gauge bosons are "gauginos". As a result of the electroweak symmetry breaking all neutral higgsinos and gauginos mix with each other to form the Majorana fermions, called neutralinos. Similarly the charged higgsinos and gauginos mix and form two massive Dirac fermions, "charginos".

The reader can refer to Table B.1 for a more complete legenda of SM particle and corresponding superparticles.

\subsubsection{Constraints on SUSY models}

The most general MSSM lagrangian contains 124 entries to be determined by the experiment, including the SM parameters. Such a large number of free physical parameters are quite a challenge to handle in a theory expecially when this theory has to be used to make any kind of experimental predictions. In fact, these variables can in principle take values in wide ranges. One common approach is to make a sufficient number of assumptions on the parameter space, typically motivated by a theorethical framework, or by particular physics scenarios (more often regarding the SUSY breaking mechanism), in order to end up with a reduced set of parameters; this reduced set of variables may be defined at some specific energy scale, for instance at high energy scale where enhanced symmetries are supposed to exactly hold. This thesis will refers to such a specific high energy scenario, namely the minimal supergravity (mSUGRA). In mSUGRA, the boundary conditions are 


\begin{tabular}{|c|c|c|c|c|c|}
\hline \multirow{2}{*}{\multicolumn{2}{|c|}{$\begin{array}{l}\text { SM } \\
\text { particles/fields }\end{array}$}} & \multicolumn{4}{|l|}{$\begin{array}{l}\text { SUSY } \\
\text { partners }\end{array}$} \\
\hline & & $\begin{array}{l}\text { Interaction } \\
\text { eigenstates }\end{array}$ & & $\begin{array}{l}\text { Mass } \\
\text { eigenstates }\end{array}$ & \\
\hline$q$ & "quark & $\tilde{q}_{L, R}$ & squark & $\tilde{q}_{1,2}$ & squark \\
\hline$l$ & lepton & $\tilde{l}_{L, R}$ & slepton & $\tilde{l}_{1,2}$ & slepton \\
\hline$\nu$ & neutrino & $\tilde{\nu}$ & sneutrino & $\tilde{\nu}$ & sneutrino \\
\hline$g$ & gluon & $\tilde{g}$ & gluino & $\tilde{g}$ & gluino \\
\hline$W^{ \pm}$ & $W$-boson & $\tilde{W}^{ \pm}$ & wino & & \\
\hline$H^{-}$ & Higgs boson & $\tilde{H}_{1}^{-}$ & higgsino & $\tilde{\chi}_{1,2}^{ \pm}$ & charginos \\
\hline$H^{+}$ & Higgs boson & $\tilde{H}_{2}^{+}$ & higgsino & & \\
\hline$B$ & $B$-field & $\tilde{B}^{2}$ & bino & & \\
\hline$W^{3}$ & $W^{3}$-field & $\tilde{W}^{3}$ & wino & $\tilde{\chi}_{1,2,3,4}^{0}$ & neutralinos \\
\hline$H_{1}^{0}$ & Higgs boson & $\tilde{H}_{1}^{0}$ & higgsino & & \\
\hline$H_{2}^{0}$ & Higgs boson & $\tilde{H}_{2}^{0}$ & higgsino & & \\
\hline$H_{3}^{0}$ & Higgs boson & & & & \\
\hline
\end{tabular}

Table 1.1: The SM particles and their superpartners in the MSSM.

defined at the GUT scale requiring a gaugino soft breaking unified mass equal to $m_{1 / 2}$ as well as the universality of sfermions and Higgs mass terms, all set to a common value $m_{0}$. Finally universality of trilinear couplings $\left(A_{0}\right)$ is also assumed at the GUT scale. Requiring the proper electroweak radiative symmetry breaking and indicating with $\tan \beta$ the ratio of the up and down type Higgs vacuum expectation values, one is left with a theory defined by a set of four parameters and one sign

$$
\tan \beta, m_{1 / 2}, m_{0}, A_{0}, \operatorname{sign} \mu
$$

where $\mu$ is the coupling of the Higgs mass term in the supersymmetric Lagrangian. The low energy phenomenology is then dictated by the resulting supersymmetric soft breaking terms as derived when running down at the electroweak energy scale.

\subsubsection{Neutralinos and Charginos}

The SUSY mass spectrum can be deduced through a standard procedure from the various particle mass matrices originating from the superposition of all the contributions appearing in the Lagrangian terms. Since this thesis focus on the production of charginos and neutralinos, their mass matrix is presented in more detail. Neutralinos are defined as the mass eigenstates of the superposition of the fermionic partners of the gauge and Higgs bosons. In the basis $\left(\tilde{B}, \tilde{W}_{3}, \tilde{H}_{1}^{0}, \tilde{H}_{2}^{0}\right)$, the mass matrix is given by 


$$
M_{\tilde{\chi}^{0}}=\left(\begin{array}{cccc}
M_{1} & 0 & -\frac{g^{\prime} v_{1}}{\sqrt{2}} & -\frac{g^{\prime} v_{2}}{\sqrt{2}} \\
0 & M_{2} & -\frac{g v_{1}}{\sqrt{2}} & -\frac{g v_{2}}{\sqrt{2}} \\
-\frac{g^{\prime} v_{1}}{\sqrt{2}} & -\frac{g v_{1}}{\sqrt{2}} & 0 & -\mu \\
-\frac{g^{\prime} v_{2}}{\sqrt{2}} & -\frac{g v_{2}}{\sqrt{2}} & -\mu & 0
\end{array}\right)
$$

where $v_{1,2}$ are the vacuum expectation values of the $H_{1,2}$ Higgs doublets and $M_{1,2}$ are the mass parameters of the bino and wino respectively. The neutralino mass matrix can be diagonalized, providing the mass eigenstates, the four neutralinos, which can be expressed as

$$
\tilde{\chi}_{i}^{0}=N_{i 1} \tilde{B}+N_{i 2} \tilde{W}^{3}+N_{i 3} \tilde{H}_{1}^{0}+N_{i 4} \tilde{H}_{2}^{0}
$$

The quantity $\left|N_{11}\right|$ can be defined as the bino fraction of the lighest neutralino, the wino fraction the quantity $\left|N_{12}\right|$, and finally the higgsino fraction the quantity $\sqrt{\left|N_{13}\right|^{2}+\left|N_{14}\right|^{2}}$. The mass matrix for the charged Higgs and gauge boson superpartners can be expressed as

$$
M_{\tilde{\chi}^{ \pm}}=\left(\begin{array}{cc}
M_{2} & g v_{2} \\
g v_{1} & \mu
\end{array}\right)
$$

If the lighest neutralino is wino-like, i.e. if it has a dominant wino fraction, it is also approximately degenerate in mass with the lighest chargino; similarly, if it is higgsino-like, there will be a quasi-degeneracy both with the next-to-lighest neutralino and with the lighest chargino. This spectral feature yields important consequences at both the level of relic abundance computations and SUSY searches at hadron colliders.

\section{Neutralinos and Charginos leptonic decay mode}
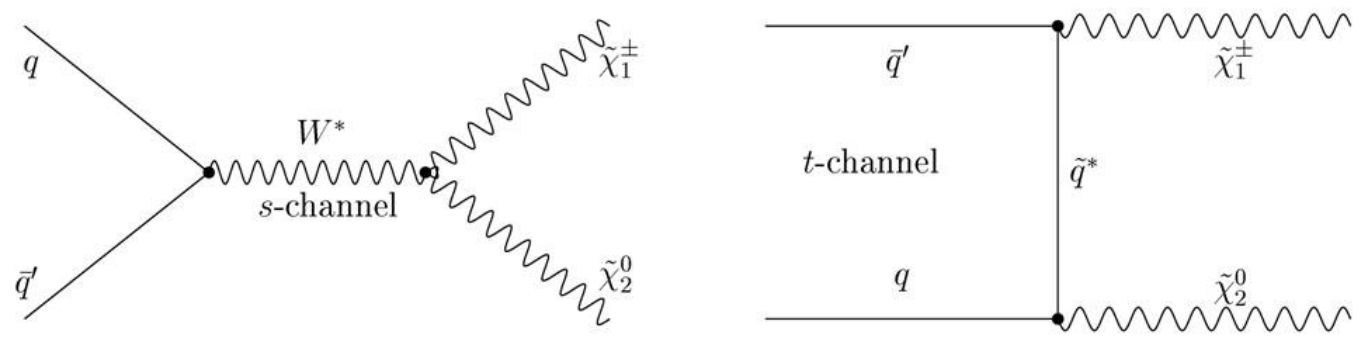

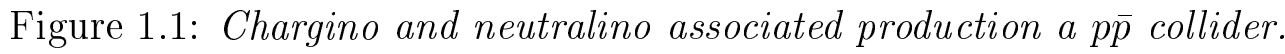

There are two different techniques mainly used for finding evidence of new physics. The first one consists in examining well known and predicted spectra for anomalies; the second one consists in searching for previously unpredicted signals. This thesis is based on the latter technique and focuses on a search for evidence of associated production of $\tilde{\chi}_{1}^{ \pm}$and $\tilde{\chi}_{2}^{0}$. The two dominating production modes are the $s$-channel, via virtual $W^{ \pm}$ 
exchange, and the $t$-channel, via virtual $\tilde{q}$ exchange (Fig. 1.1). These two modes interfere destructively. A scenario with large $\tilde{q}$ mass which suppresses the destructive $t$-channel leads to a higher production cross section. The final states interesting for this analysis are those characterized by the presence of three charged leptons which arise from the leptonic decay modes of $\tilde{\chi}_{1}^{ \pm}$and $\tilde{\chi}_{2}^{0}$ illustrated in Fig. 1.2- 1.3 .

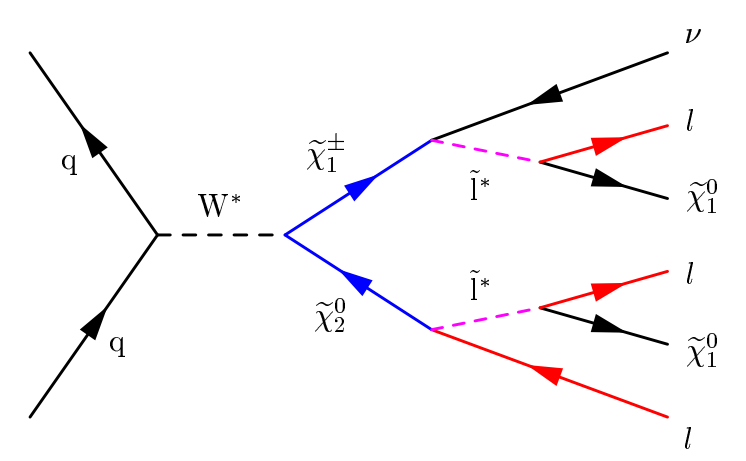

Figure 1.2: Chargino and neutralino associated production decaying via sleptons.

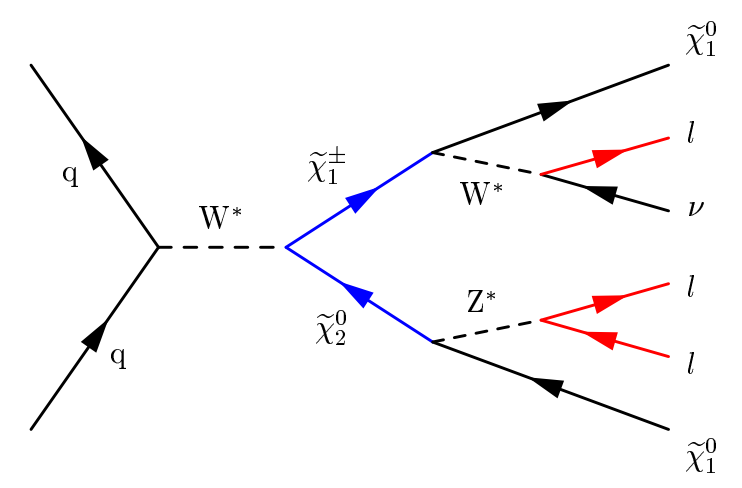

Figure 1.3: Chargino and neutralino associated production decaying via gauge bosons.

If the $\tilde{l}$ mass is smaller than the $\tilde{\chi}_{2}^{0}$ mass the predominant decay mode is via real sleptons; in this case the $\tilde{l}-\tilde{\chi}_{2}^{0}$ mass differences are important for the efficiency of the selection. For sufficiently large mass difference leptons originating from the neutralino decay have large enough momentum to be detected. On the other hand if the $\tilde{l}$ mass is greater than the neutralino mass, the decay occurs via virtual $W^{ \pm}$and $Z$ or virtual sleptons. For slepton masses comparable to the neutralino mass, the contribution of the virtual slepton cascades is large, increasing the branching fraction into leptons. For slepton masses significantly larger than the neutralino mass, the slepton cascades are in general suppressed and the dominant contribution comes from the $\tilde{\chi}$ decaying into gauge bosons. In both cases, the momentum range in which the leptons are produced depends on the mass differences $m_{\tilde{\chi}_{1}^{ \pm}}-m_{\tilde{\chi}_{1}^{0}}$ and $m_{\tilde{\chi}_{2}^{0}}-m_{\tilde{\chi}_{1}^{0}}$ : for small mass differences, final states with low momentum leptons are favoured and lepton detection becomes more challenging. In addition to the three leptons, the final state is also characterized by a neutrino and two $\tilde{\chi}_{1}^{0}$, where the lighest neutralino is assumed to be the LSP and thus invisible to detection. 
Therefore, the presence of the neutrino and the LSPs manifest themselves as missing energy in the event which can vary from a small to a substantial amount due to the fact that the directions of the escaping neutrino and two $\tilde{\chi}_{1}^{0}$ are completely uncorrelated. The trilepton + missing energy is a very distintive signature with quite small SM backgrounds at hadron colliders.

\section{Current Limits}

In the last ten years several searches aimed at finding evidence for new physics have been performed. Supersymmetric theories have been tested both at LEP2 and the TEVATRON (Run I and Run II) with the latest results from TEVATRON Run II. In the following a brief review of the most interesting results regarding chargino and neutralino searches is presented.

LEP2. All four LEP experiments (ALEPH, DELPHI, L3, OPAL) have searched for charginos in the data taken at the centre-of-mass energies up to $209 \mathrm{GeV}$ ([10]). The experiments analyzed several chargino decays modes: final states with leptons, leptons plus jets and jets only. Because the LEP experiments were dealing with much cleaner events than those produced at hadron colliders they were able to study several channels in addition to lepton-only signatures. Compared to the SM backgound expectation, no excess was observed in this data. Limits could therefore be derived. For the interpretation of the result, cross sections and branching ratios were calculated in the framework of the MSSM. Unification of gaugino masses at the GUT scale was also assumed, resulting in a scenario with fixed masses and field content of the charginos and neutralinos. Finally, the results from the different channels were combined under the assumption that the charginos decay via a virtual $W^{ \pm}$. Based on $35.2 \mathrm{pb}^{-1}$, for a mass parameter $m_{\tilde{\nu}}>300 \mathrm{GeV} / \mathrm{c}^{2}$, the exclusion limit set by LEP2 (Fig. 1.4) for the chargino mass is quoted as:

$$
\tilde{\chi}_{1}^{ \pm} \geq 103.5 \mathrm{GeV} / \mathrm{c}^{2}
$$

TEVATRON. At hadron colliders the biggest challenge is to deal with the huge amount of QCD events produced. This becomes even more critical when one searches for new physics. That is why the search for chargino and neutralino production at the TEVATRON has been performed focusing on multilepton final states which lead to clean signatures and manageable QCD backgrounds.

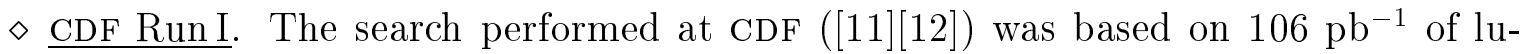
minosity collected during the 1993-1995 period. In the framework of the MSSM, the search was for the direct production of $\tilde{\chi}_{1}^{ \pm} \tilde{\chi}_{2}^{0}$ via virtual $W^{ \pm}$and squark exchange diagrams decaying into four trilepton final states $\left(e^{+} e^{-} e, e^{+} e^{-} \mu, e \mu^{+} \mu^{-}\right.$, $\left.\mu^{+} \mu^{-} \mu\right)$. Events were selected with a central electron with $E_{T}^{e}>11 \mathrm{GeV}$ or a central muon with $p_{T}^{\mu}>11 \mathrm{GeV} / \mathrm{c}$ requiring two additional leptons (excluding taus) with $E_{T}^{e}>5 \mathrm{GeV}$ or $p_{T}^{\mu}>4 \mathrm{GeV} / \mathrm{c}$. The main requirements (hereafter referred to as "standard" requirements) imposed on the selected events were:

* cosmic and conversion removal mainly based on tracking information;

$\star$ calorimeter isolation of leptons to remove backgrounds from $t \bar{t}$ and $b \bar{b}$; 


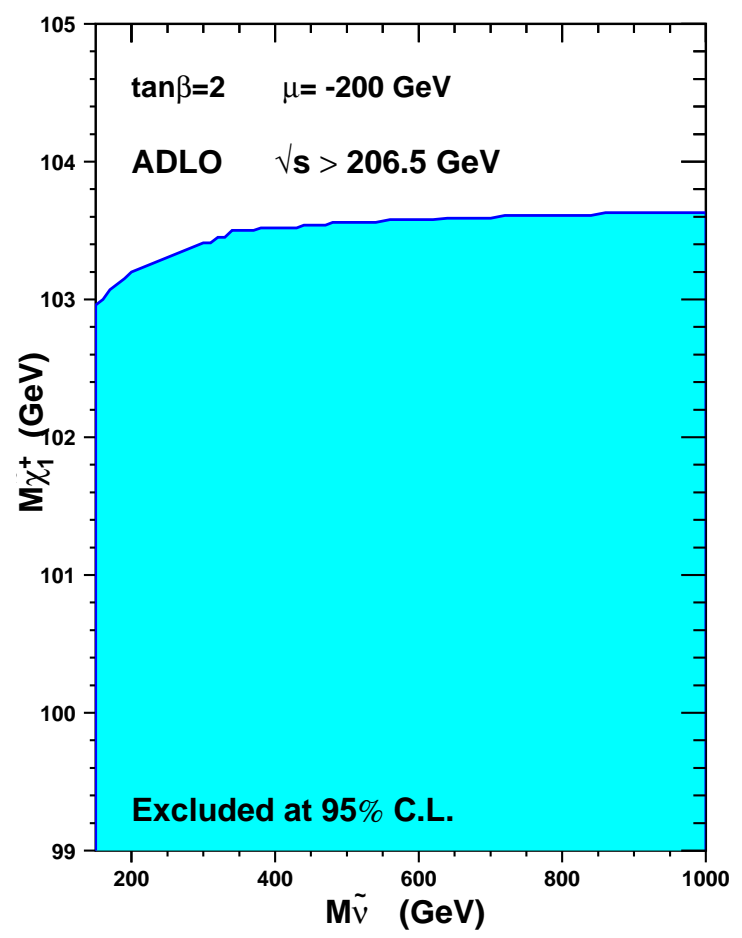

Figure 1.4: Chargino Mass Limit versus $m_{\tilde{\nu}}$ set by the LEP2.

* minimal separation between each pair of leptons in the event;

* the maximal difference between leptons in azimuthal angle must be less than an upper value in order to remove events with back-to-back topology like DrellYan events and remaining cosmic rays;

$\star$ events containing a same flavor $l^{+} l^{-}$pair with invariant mass in the regions of the resonances $J / \psi, Y, Z$ were removed;

* the missing energy $\mathbb{E}_{T}>15 \mathrm{GeV}$ requirement for the presence of the neutrino and the LSPs in the final state kills backgrounds from $b \bar{b}$ and Drell-Yan processes.

No events passed the analysis selection consistently with the SM background prediction. Therefore no evidence for chargino-neutralino production was observed and limits were set within the framework of the MSSM models assuming $M_{\tilde{\chi}_{1}^{ \pm}} \approx M_{\tilde{\chi}_{2}^{0}} \approx$ $2 M_{\tilde{\chi}_{1}^{0}}$. The strongest limit was:

$$
\begin{gathered}
\sigma_{\tilde{\chi}_{1}^{ \pm} \tilde{\chi}_{2}^{0}} \operatorname{BR}\left(\tilde{\chi}_{1}^{ \pm} \tilde{\chi}_{2}^{0} \rightarrow 31+\mathrm{X}\right)<0.34 \mathrm{pb} \\
M_{\tilde{\chi}_{1}^{ \pm}} \geq 81.5 \mathrm{GeV} / \mathrm{c}^{2} \\
M_{\tilde{\chi}_{2}^{0}} \geq 82.2 \mathrm{GeV} / \mathrm{c}^{2}
\end{gathered}
$$

for $\tan \beta=2, \mu=-600 \mathrm{GeV} / \mathrm{c}^{2}$ and $M_{\tilde{q}}=M_{\tilde{g}}$. In Fig. $1.5 \sigma \times \mathrm{BR}$ versus the $\tilde{\chi}_{1}^{ \pm}$ mass is presented for representative points in the MSSM parameter space compared with the D0 Run I limit. 


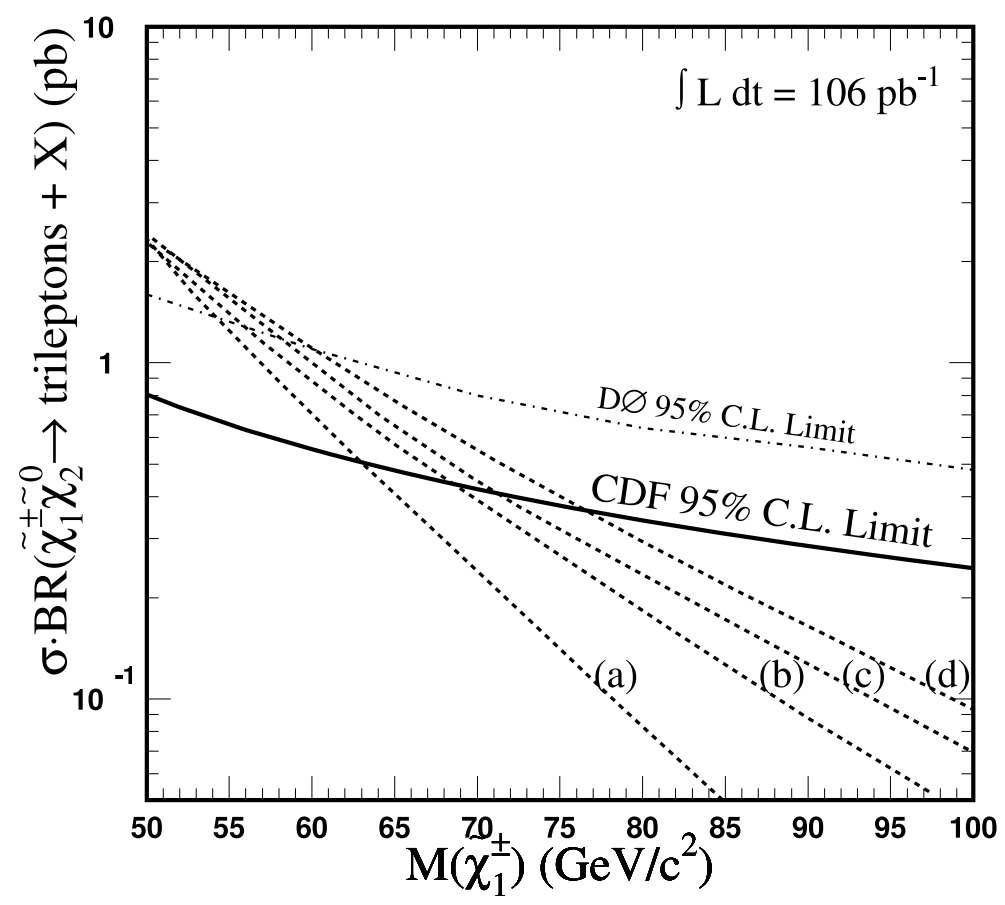

Figure 1.5: $\sigma \times \mathrm{BR}$ versus $\tilde{\chi}_{1}^{ \pm}$: limits set at $\mathrm{CDF}$ compared with $\mathrm{D} 0$ results during Run I. Dashed lines correspond to the theoretical prediction for four different choices of the squark masses: (a) $m_{\tilde{q}}=m_{\tilde{g}}$, (b) $m_{\tilde{q}}=1.2 m_{\tilde{g}}$, (c) $m_{\tilde{q}}=1.5 m_{\tilde{g}}$, (d) $m_{\tilde{q}}=2.0 m_{\tilde{g}}$.

$\diamond$ D0 Run II. Following up on the work started during Run I, the TEVATRON experiments are continuing searches for chargino and neutralino associated productions in trileptons this time with much more integrated luminosity at their disposal. The latest results from TEVATRON on chargino mass limits comes from the D0 experiment ([13][14][15][16]). The analysis is based on $320 \mathrm{pb}^{-1}$ collected with the D0 detector between 2002 and 2004. All leptons $(e, \mu, \tau)$ are considered in the final states: the inclusion of taus leads to higher leptonic branching fraction as illustrated in Fig. 1.6 in particular in scenarios with high values of $\tan \beta$. The optimization of cuts is based on signals inspired by minimal supergravity with $\tilde{\chi}_{1}^{ \pm}, \tilde{\chi}_{2}^{0}$ and $\tilde{l}$ in the range $110-130 \mathrm{GeV} / \mathrm{c}^{2}$. As a representative example, a signal sample is used with $m_{0}=84 \mathrm{GeV} / \mathrm{c}^{2}, m_{1 / 2}=176 \mathrm{GeV} / \mathrm{c}^{2}, \tan \beta=3, \mu>0$ and no slepton mixing, which corresponds to a $\tilde{\chi}_{1}^{ \pm}=110 \mathrm{GeV} / \mathrm{c}^{2}$ and $\sigma \times \mathrm{BR}=0.265 \mathrm{pb}$. Six different selections are defined depending on the lepton content of the final state: eel, $\mu \mu l, \mu^{ \pm} \mu^{ \pm}$, $e \mu l, e \tau l, \mu \tau l$. The hadronic decay of tau is identified by using a neural net. Each selection requires two identified leptons with minimal momenta: $p_{T, 1}>10 \mathrm{GeV} / \mathrm{c}$ and $p_{T, 2}>5 \mathrm{GeV} / \mathrm{c}$. The third lepton is an isolated track originated from the same vertex as the two identified leptons with a track isolation condition efficient also for hadronic decay of tau leptons. In addition to "standard" requirements, those listed above for the CDF Run I result, further background reduction is achieved by:

$\star$ removing events in which the missing energy is aligned with a lepton since imbalance in transverse energy can be generated by mismeasurements of jet or lepton energies;

* removing events containing a jet with small "significance" (a variable which 
gives an estimate of the impact of fluctuations of jet energy depositions on the missing energy);

* removing events with large $H_{T}$, defined as the scalar sum of the transverse energies of all jets, which rejects background from $t \bar{t}$;

* a combined cut on the product of missing energy and third track transverse momentum optimally reduces the remaining background, which tends to have low values of both these variables.

After all cuts, the expected background is dominated by multijet and di-boson processes. Combining all six selections, a total background of $3.85 \pm 0.57$ (stat) \pm 0.49 (syst) events is expected and 4 events are observed in the data after the whole selection. Therefore, good agreement between data and expectation from SM processes is obtained and no evidence for associated production of chargino and neutralino is observed. Assuming the mSUGRA-inspired mass relation $m_{\tilde{\chi}_{1}^{ \pm}} \approx m_{\tilde{\chi}_{2}^{0}} \approx$ $2 m_{\tilde{\chi}_{1}^{0}}$ and no slepton mixing, the limit on the $\sigma \times \mathrm{BR}$ is a function of $\tilde{\chi}_{1}^{ \pm}$and $m_{\tilde{l}}$ with a relative small dependence on the other SUSY parameters. The results can then be interpreted in more general SUSY scenario as long as the above assumptions are preserved. The interpretation of the result is summarized in Fig. 1.7. The leptonic branching fractions of chargino and neutralino depend on the relative contribution from slepton and $W / Z$-exchange diagrams:

$\star$ large- $m_{0}$ scenario. $W / Z$-exchange is dominant at large $m_{\tilde{l}}$ resulting in relatively small leptonic branching fractions. As can be desumed from Fig. 1.7 the performed analysis shows no sensitivity for this scenario.

* 3l-max scenario. The leptonic branching fraction is maximally enhanced for $m_{\tilde{l}} \geq m_{\tilde{\chi}_{2}^{0}}$. Decays into leptons can even be dominant if sleptons are light enough that two-body decays are possible. In this scenario the chargino mass limit corresponds to $116 \mathrm{GeV} / \mathrm{c}^{2}$.

* heavy squarks scenario. The chargino-neutralino production depends on the squark masses due to the negative interference of the $t$-channel squark exchange graph, hence the cross section is maximal in the limit of large squark masses. In this scenario the chargino mass limit corresponds to $128 \mathrm{GeV} / \mathrm{c}^{2}$.

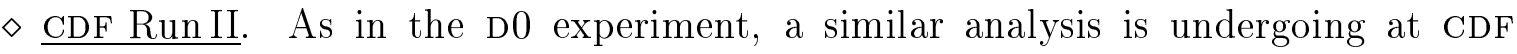
searching for trileptons (electrons and muons only) events coming from chargino and neutralino associated production ([17][18][19][20]). Several channels have been studied: the luminosity used for this search varies from $300 \mathrm{pb}^{-1}$ to $750 \mathrm{pb}^{-1}$ (collected between 2002 and 2005) depending on the channels. The analyses can be subdivided into high- $p_{T}$ and low- $p_{T}$ analyses depending on the threshold asked for the leading lepton of the event: high- $p_{T}$ analyses (leading lepton $p_{T}>20 \mathrm{GeV} / \mathrm{c}$ ) $e e l, \mu e l, \mu \mu l$ where $l=e, \mu$ is an identified lepton; low- $p_{T}$ analyses (leading lepton $p_{T}>10 \mathrm{GeV} / \mathrm{c}$ ) eel where $l$ is an isolated track, $\mu \mu l$ where $l=e, \mu$.

Similarly to the D0 Run II analyses, further cuts are applied after the "standard" selection, also depending on the specific channel considered. The search has been performed as a blind analysis aimed at understanding the SM backgrounds before looking at the kinematical region where the SUSY signal is enhanced w.r.t. SM 


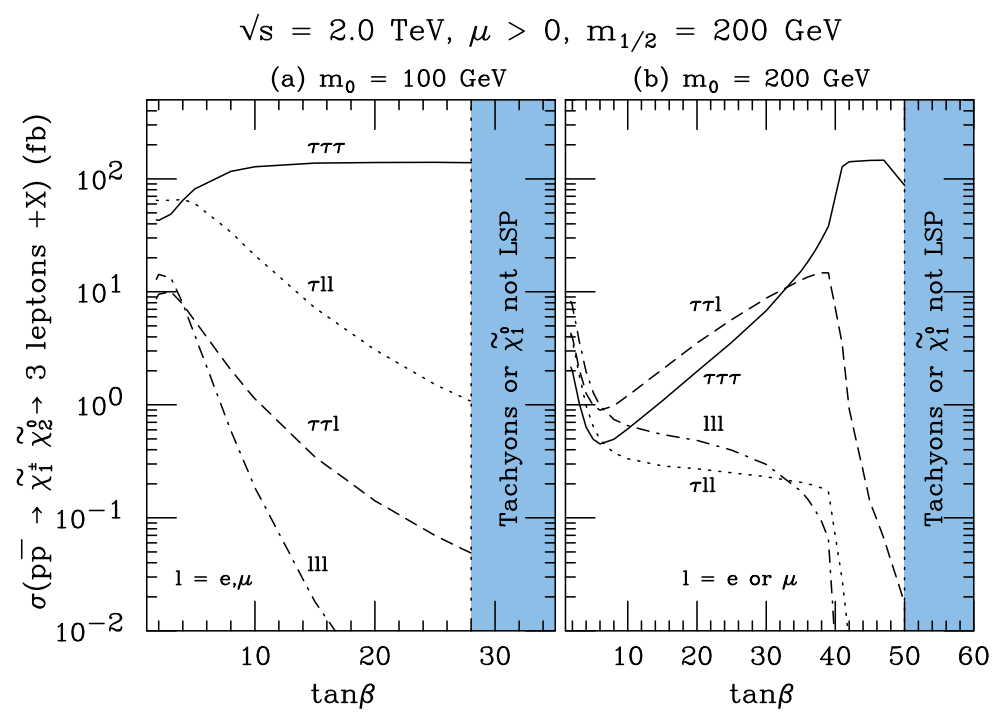

Figure 1.6: Cross section for associated production of chargino and neutralino as function of $\tan \beta$.

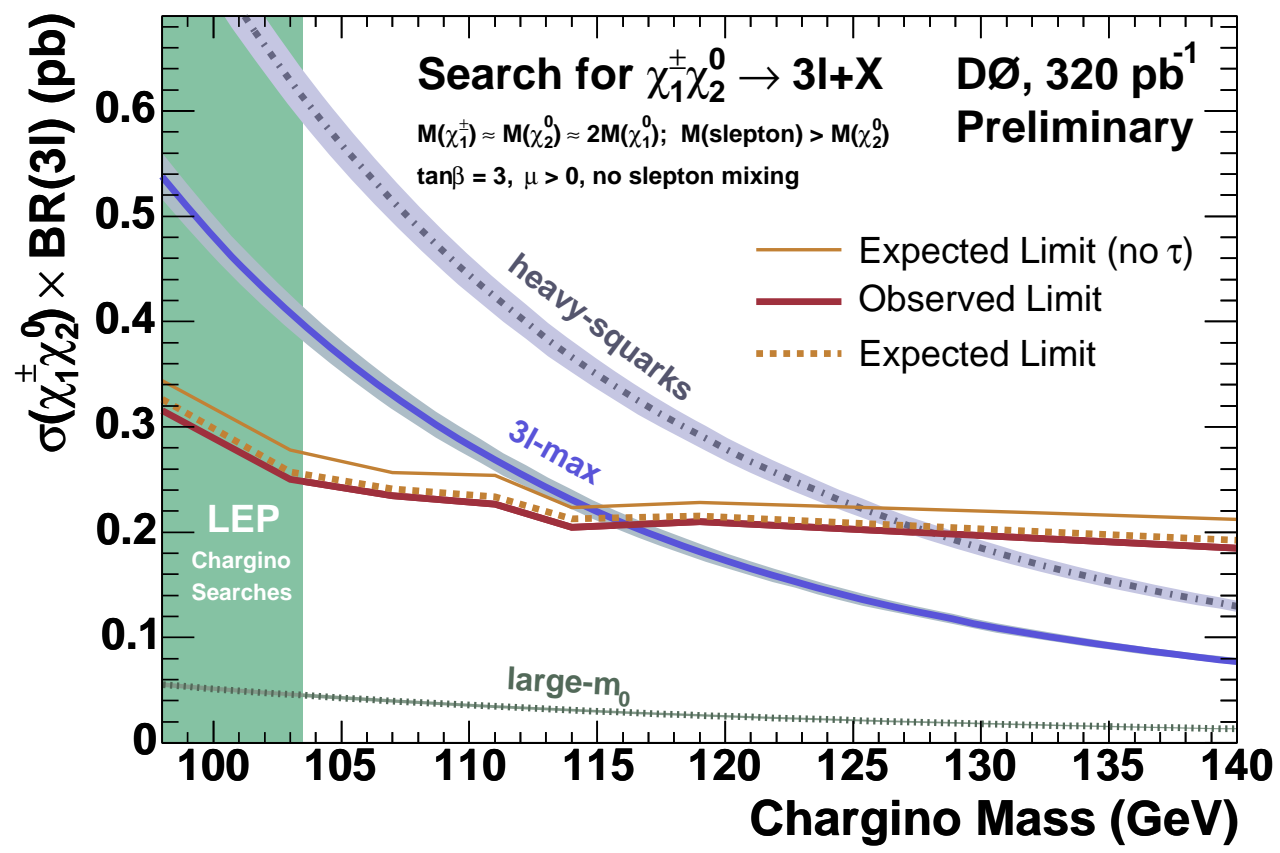

Figure 1.7: $\sigma \mathrm{BR}$ versus $\tilde{\chi}_{1}^{ \pm}$: limits set at $\mathrm{D} 0$ during Run II. 
processes. The SM predicted distributions are therefore compared to data events in control regions defined by reversing one or more analysis cuts (for instance the invariant mass and the missing energy cuts). After understanding all considered control regions, data and background+signal MC are directly compared in the SUSY signal region. All the channels show no discrepancy from the prediction of the SM processes and the results obtained from each channel are summarized in Tab. 1.2.

A preliminary study is undergoing to exclusively combine all the analyses in order to set a limit on the chargino mass. The analysis presented in this thesis will contribute in the CDF low- $p_{T}$ analyses focusing on the $e \mu+l$ channel where $l=e, \mu$.

\begin{tabular}{|l|c|c|c|}
\hline Analysis & Luminosity & Expected SM events & Observed data events \\
\hline \hline high- $p_{T} e e$ & $346 \mathrm{pb}^{-1}$ & $0.17 \pm 0.03($ stat $) \pm 0.04($ syst $)$ & 0 \\
high- $p_{T} \mu \mu$ & $745 \mathrm{pb}^{-1}$ & $0.64 \pm 0.11($ stat $) \pm 0.14($ syst $)$ & 1 \\
high- $p_{T} \mu e($ central $)$ & $745 \mathrm{pb}^{-1}$ & $0.28 \pm 0.03($ stat $) \pm 0.04($ syst $)$ & 0 \\
high- $p_{T} \mu e($ forward $)$ & $680 \mathrm{pb}^{-1}$ & $0.36 \pm 0.05($ stat $) \pm 0.05($ syst $)$ & 0 \\
low- $p_{T} e e$ & $607 \mathrm{pb}^{-1}$ & $0.42 \pm 0.08($ stat $) \pm 0.06($ syst $)$ & 1 \\
low- $p_{T} \mu \mu$ & $312 \mathrm{pb}^{-1}$ & $0.04 \pm 0.01($ stat $) \pm 0.01($ syst $)$ & 0 \\
\hline
\end{tabular}

Table 1.2: Standard Model prediction compared with observed data obtained at CDF Run II for the chargino-neutralino search in trileptons. The high- $p_{T} \mu e(c e n t r a l)$ analysis requires the electron to be identified in the central region, while in the high-p $p_{T} \mu e$ (forward) selection the electron must be detected in the forward calorimeter. 



\section{Bibliography}

[1] F. Mandl, G. Shaw, Quantum Field Theory, John Wiley \& Sons (1996).

[2] M. E. Peskin, D. V. Schroeder, An Introduction to Quantum Field Theory, AddisonWesley (1997).

[3] A. Pich, The Standard Model of electroweak interactions, HEP-PH/0502010, Feb 2005.

[4] Particle Data Group, Review of particle physics, K. Hagiwara et al., Phys. Rev. D 66, $010001(2002)$

[5] H. Murayama, Supersymmetry phenomenology HEP-PH/0002232, Mar 2000.

[6] S.P. Martin, A Supersymmetry primer HEP-PH/9709356, Apr 1999.

[7] D.J.H.Chung et Al., The Soft Supersymmetry-Breaking Lagrangian: Theory and Applications HEP-PH/0312374, Dec 2003.

[8] M.E. Peskin, Beyond the Standard Model HEP-PH/9705479, 1997.

[9] M. Drees, An introduction to supersymmetry HEP-PH/9611409, 1996.

[10] The LEP2 experiments, Combined LEP Chargino Results http://lepsusy.web.cern.ch/lepsusy/www/inos_moriond01/charginos_pub.html, 2001.

[11] The CDF collaboration, Phys. Rev. Lett. 80, 24 (1998).

[12] B. Tannenbaum et Al., Update to the Run 1A+1B Chargino-Neutralino Search Using Trilepton Events CDF internal note 4188, Aug 1997.

[13] The D0 collaboration, Phys. Rev. Lett. 95, 151805 (2005).

[14] The D0 collaboration, Search for the Associated Production of Charginos and Neutralinos in the $e+\tau^{\text {had }}+l$ Final State D0 note 4741, Mar 2005.

[15] The D0 collaboration, Search for the Associated Production of Charginos and Neutralinos in the $\mu+\tau+l$ Final State D0 note 4742, Mar 2005.

[16] The D0 collaboration, Search for the Associated Production of Chargino and Neutralino in Final State with Three Leptons involving $\tau$ Leptons D0 note 4740, Mar 2005. 
[17] M. Gold et Al., Search for the chargino-neutralino production at the $\mu \mu+l$ decay channel at $312 p b^{-1}$ CDF internal note 8101, Feb 2006.

[18] D. Bortoletto et Al., Search for the associated production of chargino and neutralino in the final state with one muon and two additional leptons CDF internal note 8114, Mar 2006.

[19] S. Dube et Al., Search for Chargino-Neutralino Production in mSUGRA Model in a Di-electron + Track Channel CDF internal note 8098, Feb 2006.

[20] M. Griffiths et Al., Search for associated production of chargino and neutralino in trilepton final state using the high-p $p_{T}$ electron trigger CDF internal note 7868, Sep 2005. 


\section{Chapter 2}

\section{Tevatron and CDF for Run II}

The TEVATRON accelerator complex is located in Fermilab, an international accelerator laboratory about 30 miles West from Chicago (Illinois). Here protons and antiprotons are accelerated and collide at two intersection points of the $6.3 \mathrm{~km}$ main ring where two multipurpose detectors, CDF and D0, have been built to study the products of the particle-antiparticle collisions at $1.96 \mathrm{TeV}$ center of mass energy.

Until start-up of LHC, the TEVATRON is the only machine where TeV-scale collisions are observable, making this accelerator the most powerful tool presently available for high energy physics searches.

The machine and detectors set up for Run I (ended in 1996) led to the discovery of the top quark but were not optimal for Higgs searches and even less so for searches of new physics which are expected to need much more statistics in order to achieve enough sensitivity to reveal possibly interesting processes ${ }^{1}$. The ambitious physics goals that were proposed for Run II (started in 2001) therefore needed specific upgrades of both the accelerator complex and detector components.

This chapter describes the upgrades that the TEVATRON accelerator ${ }^{2}$ and the CDF detector $^{3}$ have undergone.

\subsection{The accelerator}

The number of events which are expected for a given process is given by the expression:

$$
N_{\text {evts }}=\sigma \cdot \int \mathcal{L} \mathrm{d} t
$$

where $\sigma$ is the process cross section and $\mathcal{L}$ is the instantaneous luminosity. It follows that $N_{\text {evts }}$ can be increased in three ways:

1. performing the experiment in the situation that maximizes $\sigma$;

2. augmenting the instantaneous luminosity;

3. increasing the running time of the experiment.

\footnotetext{
${ }^{1}$ whose limited production cross sections essentially reflect the smallness of the electroweak coupling constant and the high mass of the new particles involved.

${ }^{2}$ Refer to [1] for more documentation about the accelerator complex.

${ }^{3} \mathrm{~A}$ detailed description of the detector upgrades is contained in [2].
} 
Being aimed at obtaining larger statistics, the proposed upgrades for TEVATRON essentially followed the previous scheme; steps 1 and 2 respectively translated into:

$\diamond$ increasing the center of mass energy of the $p \bar{p}$ collisions;

$\diamond$ increasing the luminosity of the accelerator.

Collision energy. The Run II of TEVATRON will be characterized by a center of mass energy of $\sqrt{s}=2 \mathrm{TeV}$, about $10 \%$ higher than in Run I. This enhances production cross sections for signals which are amongst the principal physics goals of Run $\mathrm{II}^{4}$.

Luminosity. The instantaneous luminosity can be expressed in terms of:

$$
\mathcal{L} \propto \frac{f B N_{\mathrm{p}} N_{\overline{\mathrm{p}}}}{2 \pi\left(\sigma_{\mathrm{p}}^{2}+\sigma_{\overline{\mathrm{p}}}^{2}\right)}
$$

where:

$f: \quad$ beam revolution frequency,

$B$ : number of proton/antiproton bunches,

$N_{\mathrm{p}, \overline{\mathrm{p}}}$ : number of protons/antiprotons per bunch,

$\sigma_{\mathrm{p}, \overline{\mathrm{p}}}:$ transverse proton/antiproton bunch dimension at interaction point,

while the proportionality is determined by a form factor which depends on the longitudinal beam size and on its dispersion in phase space.

While maintaining the number of protons/antiprotons per bunch almost the same as in Run I, in Run II the most significant improvement towards high luminosities was achieved by increasing the number of bunches from 6 to 36. This, together with reduced bunch sizes, has led to instantaneous luminosity peaks of $\sim 2 \times 10^{32} \mathrm{~cm}^{-2} \mathrm{~s}^{-1}$ so far (see Fig. 2.1-2.2).

An important consequence of augmenting the number of bunches affects both detector operation and the consequent event reconstruction: a larger number of bunches reduces the time between collisions, which in turn implies that a shorter time is available for readout. Crossing time decreases from 3.5 $\mu$ s (Run I, 6 bunches) to 396 ns for 36 bunches in Run II. This has important consequences for the readout and triggering procedures: events collected by the detector need to be stored in memory and fed to the trigger boards through a pipeline in order to avoid dead time during processing. With increasing luminosity, more than one hard or semi-hard scattering can occur in a beam crossing. The increase in the number of bunches is aimed at compensating for the increase of luminosity.

In the following, a description of the various components of TEVATRON (see Fig. 2.3) in Run II configuration is given.

\subsubsection{Proton production and Booster}

The proton source at Fermilab comprises a $400 \mathrm{MeV}, 150 \mathrm{~m}$ long, linear accelerator (Linac) which is fed with $750 \mathrm{keV}$ negative hydrogen ions produced by a Cockcroft-Walton accelerator. Exiting from the Linac, the two electrons are stripped from the ions when they traverse a thin sheet of graphite; the protons so obtained are injected into a small synchrotron having a diameter of $150 \mathrm{~m}$ (Booster) which accelerates protons to an energy of $8 \mathrm{GeV}$. At the same time, by mean of radio frequency cavities, the Booster collects the protons into bunches, part of which are destinated for antiproton production.

\footnotetext{
${ }^{4}$ For instance, the $t \bar{t}$ production cross section is $\sim 40 \%$ higher than in the Run I scenario
} 


\section{Collider Run II Peak Luminosity}

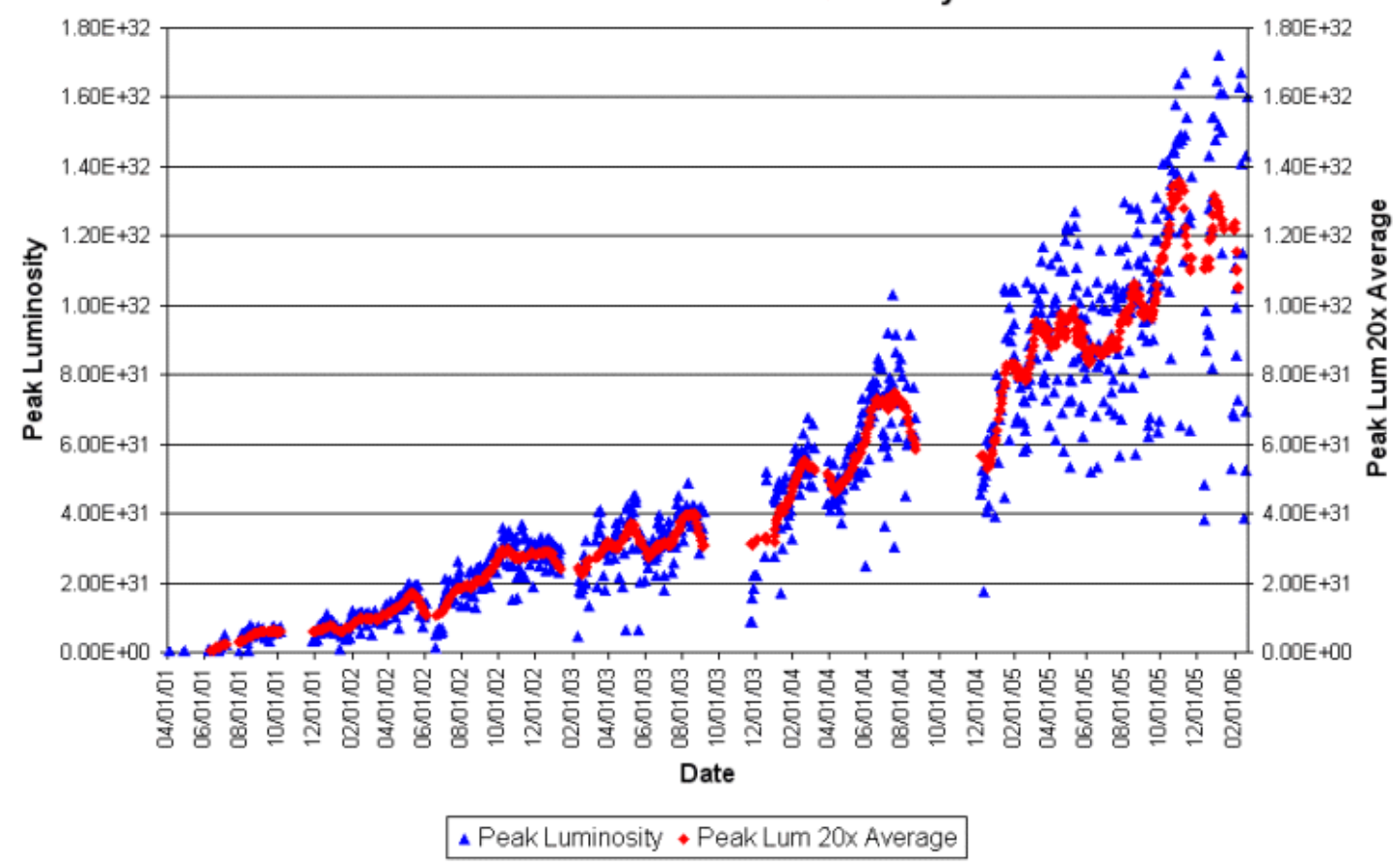

Figure 2.1: Instantaneous luminosity peak vs.time.

\section{Collider Run II Integrated Luminosity}

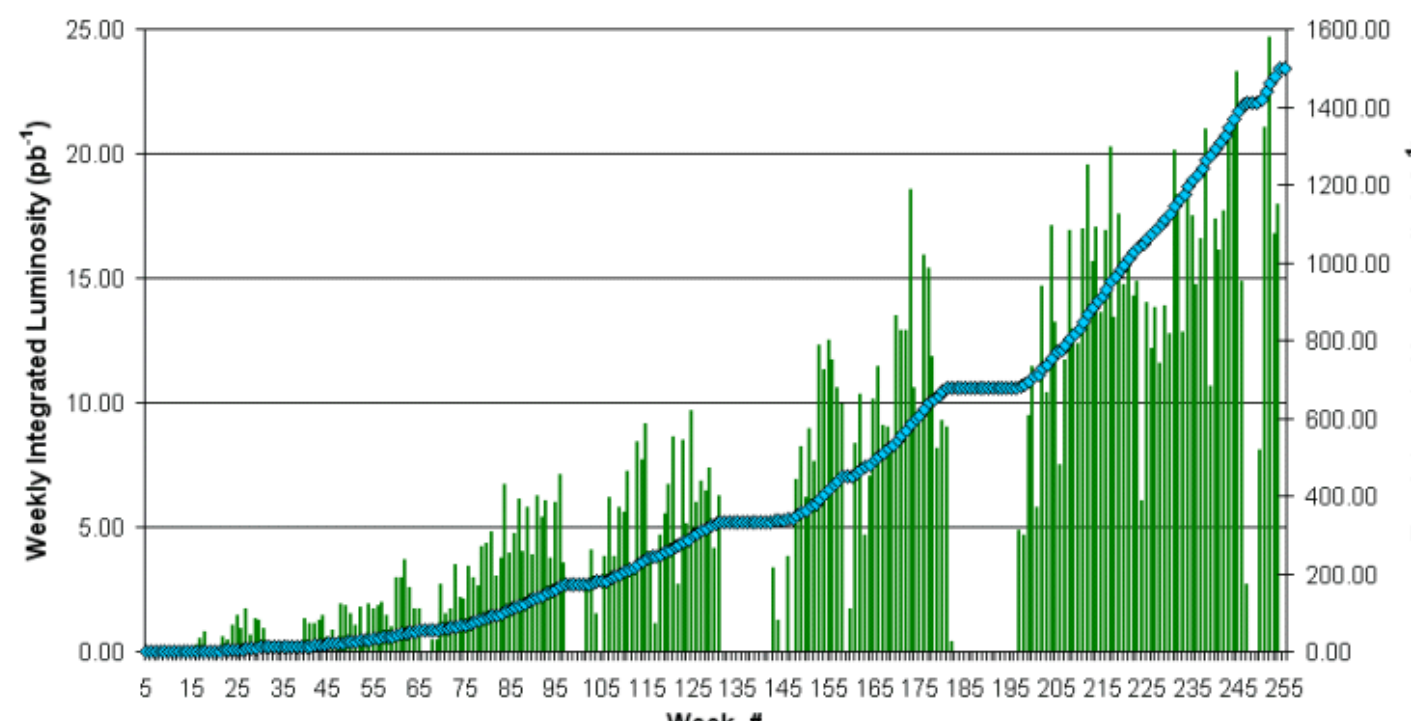

Week \#

(Week 1 starts 03/05/01)

Weekly Integrated Luminosity $\leadsto$ Run Integrated Luminosity

Figure 2.2: Integrated luminosity vs.time. 


\section{FERMILAB'S ACCELERATOR CHAIN}

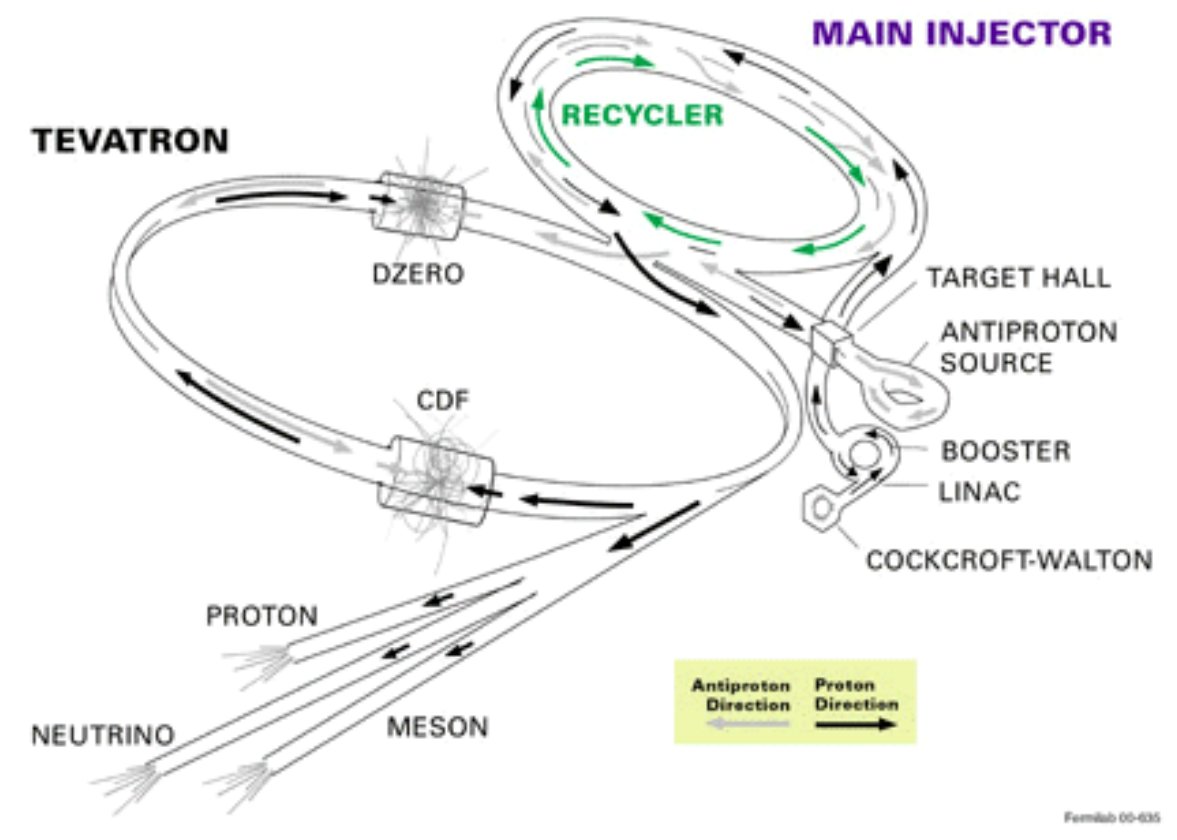

Figure 2.3: Schema of the Tevatron accelerator complex.

\subsubsection{The Main Injector}

The bunches of protons produced in the Booster are injected into the Main Injector, a $3 \mathrm{~km}$-circumference proton syncrotron, whose main function is accelerating protons and antiprotons to an energy of $150 \mathrm{GeV}$ for injection into the TEVATRON. Another important feature of the Main Injector is its ability to decelerate antiprotons for the purpose of recovering unused antiprotons when Tevatron is not in colliding mode. The Main Injector replaces the Main Ring (which was located in the same tunnel as the TEVATRON), in use during Run I, which, having been initially planned for proton acceleration in fixed target experiments, was not optimally configured as an injector for the TEVATRON due to its low phase space acceptance.

\subsubsection{Antiproton production and cooling}

Antiprotons are produced by dumping a $120 \mathrm{GeV}$ proton bunch from the Main Injector onto a nickel target ${ }^{5}$. A lithium lens collects and focuses all particles coming from the target and a bending magnet separates antiprotons from all other negatively charged particles, emerging with an energy of $\sim 8 \mathrm{GeV}$. The pulses of antiprotons are fed into the Debuncher Ring, where they are collected as a continuum and stochastically cooled; further cooling proceeds in the Accumulator, into which they are moved from the Debuncher Ring. When a sufficiently high number of antiprotons is available, they are collected into a bunch which is injected into the Recycler Ring.

\footnotetext{
${ }^{5}$ More than $6 \cdot 10^{12}$ protons strike this target every $2-3$ seconds.
} 


\subsubsection{The Recycler Ring}

The Recycler Ring serves as an antiproton accumulating ring and is located in the same cavity which hosts the Main Injector; the purpose of this device - which did not exist during Run I - is electron-cooling and storing the bunches of antiprotons produced by the Accumulator or recovered by the Main Injector until they are used again. This enables an efficient management of the antiproton bunches: on one hand, at the end of a run they are not dumped (as it used to be during Run I), while on the other - thanks to the architecture of the Recycler Ring, based upon permanent magnets - they can be stored without the risk of loosing the beam as a consequence of power losses or surges. The importance of this device is related to the limiting power of the antiproton production on the colliding procedure at FERMILAB. The recycling of antiprotons can by itself provide a factor two increase on the average integrated luminosity by recovering $75 \%$ of the antiprotons that still circulate in the TEVATRON at the end of a store ${ }^{6}$.

\subsubsection{The Tevatron}

The TEvatron is a $\sim 6 \mathrm{~km} p \bar{p}$ circular collider: oppositely charged beams are made to circulate in opposite directions within the same beam pipe. The energy of proton and antiproton bunches is raised from $150 \mathrm{GeV}$ (the nominal value of the beam energy on exiting the Main Injector) to $980 \mathrm{GeV}$ using cryogenically cooled magnets ${ }^{7}$. Once the beams reach the designed energy they are crossed in two of the twelve possible crossing points. The remaining ten are avoided by means of electrostatic separators. The two intersection points are known as the $\mathrm{B} 0(\mathrm{CDF})$ and $\mathrm{D} 0$ interaction points, where collisions actually take place.

The upgrades developed specifically for each component of the accelerator chain enable the TEVATRon to produce $p \bar{p}$ collisions at $\sqrt{s}=1.96 \mathrm{TeV}$. So far it has delived a total integrated luminosity of $\sim 1.5 \mathrm{fb}^{-1}$ during Run II (see Fig. 2.2), compared to $\sim 200 \mathrm{pb}^{-1}$ during Run I.

\subsection{The CDF II detector}

The Collider Detector at Fermilab (CDF) (see Fig. 2.4) has been upgraded in order to accommodate the increased luminosity and reduced time-interval between consecutive bunches, which require faster readout and triggering.

For these reasons all tracking devices, which are concentrated in the inner part of CDF, have been redesigned. At the same time, new readout electronics has been provided for all detector subsystems.

Furthermore, the long period of machine development (started in 1996) has permitted an increase of the detector acceptance, which has mainly involved upgrades of the tracking system, the calorimeter and the muon systems (whose enlarged geometrical coverage can be seen in fig. 2.4).

\footnotetext{
${ }^{6}$ A store is a period of time, lasting up to $\sim 30$ hours, in which the beam is kept circulating continuously in the TEVATRON; it can be a HEP store (with protons and antiprotons) or a proton-only store for studies and maintenance.

${ }^{7}$ Magnets are cooled by liquid helium $(\mathrm{T}=4 \mathrm{~K})$ and provide a $4.5 \mathrm{~T}$ filed.
} 


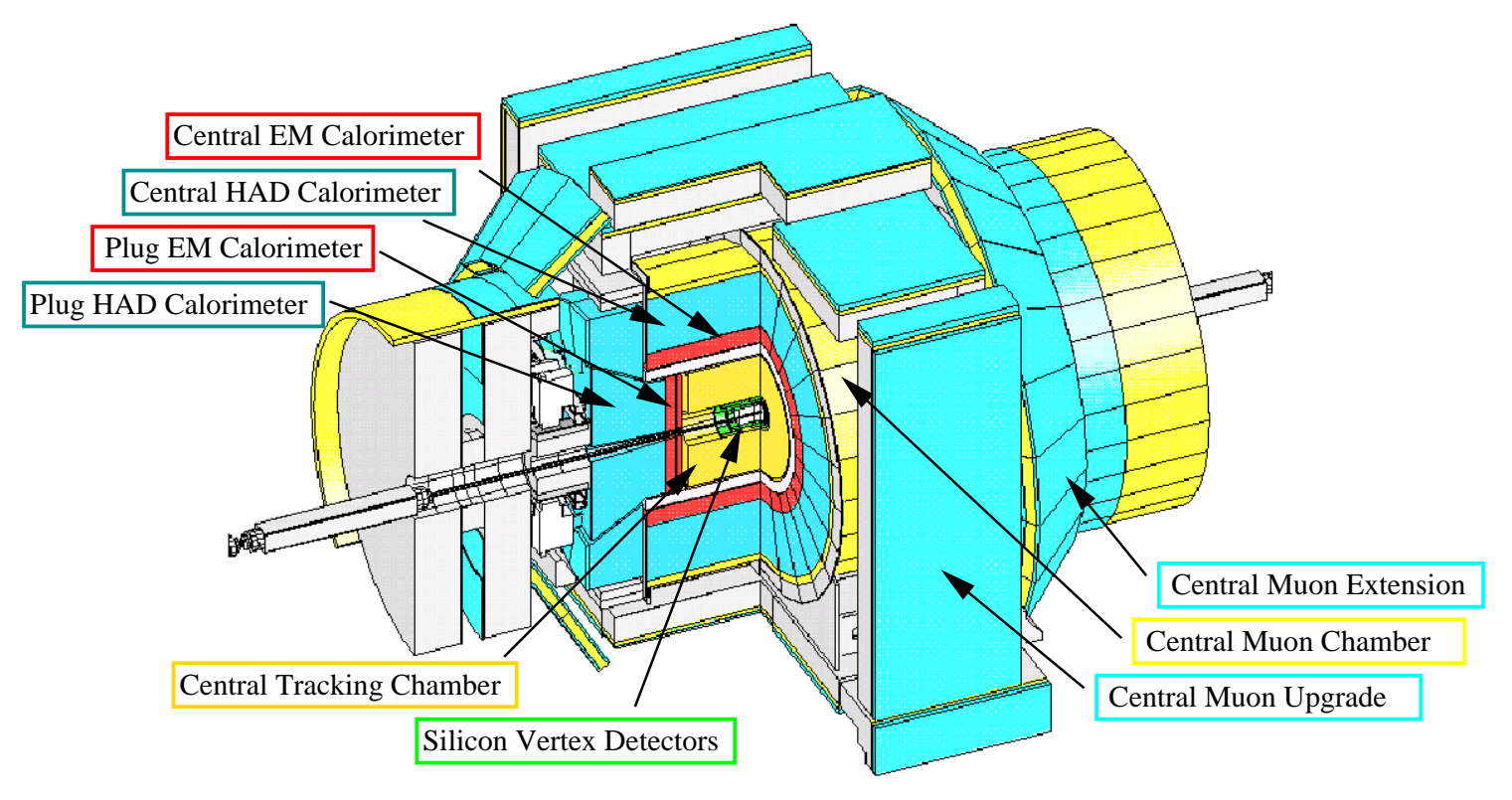

Figure 2.4: The CDF II detector.

Before giving a brief description of CDF II subdetectors, it is convenient to define the two currently used coordinate systems.

Cartesian system: the $z$ axis (East-West direction) is coincident with the beamline, its positive direction parallel to the motion of protons. The $x y$ plane contains the nominal interaction point, which coincides with the center of CDF; the $x$ axis (North-South direction) is oriented horizontally towards the outer side of the accelerator ring, while the $y$ axis is defined by requiring $(x, y, z)$ to a be right-handed system.

Polar system: the origin of the system is the same as in the Cartesian case and has the $z$ axis in common with it. The azimuthal angle $\phi$ is therefore measured in the $x y$ plane starting from the $x$ axis. The polar angle $\theta$ is measured from the positive direction of the $z$ axis. Finally, $r$ defines the transverse distance from the $z$ axis. The pseudorapidity, defined in terms of $\theta$ as:

$$
\eta=-\ln (\tan (\theta / 2))
$$

is particularly useful at hadron colliders, where events are boosted along the beamline because it transforms linearly under Lorentz-boosts:

$$
\eta \longrightarrow \eta+\tanh ^{-1} \beta
$$

(where $\beta$ is the relative velocity associated with the boost). This implies that pseudorapidity intervals, $\Delta \eta$, are relativistic invariants, preserving in this way the average particle flux per unit of $\eta$. This fact has influenced the segmentation of the calorimetric apparatus. 


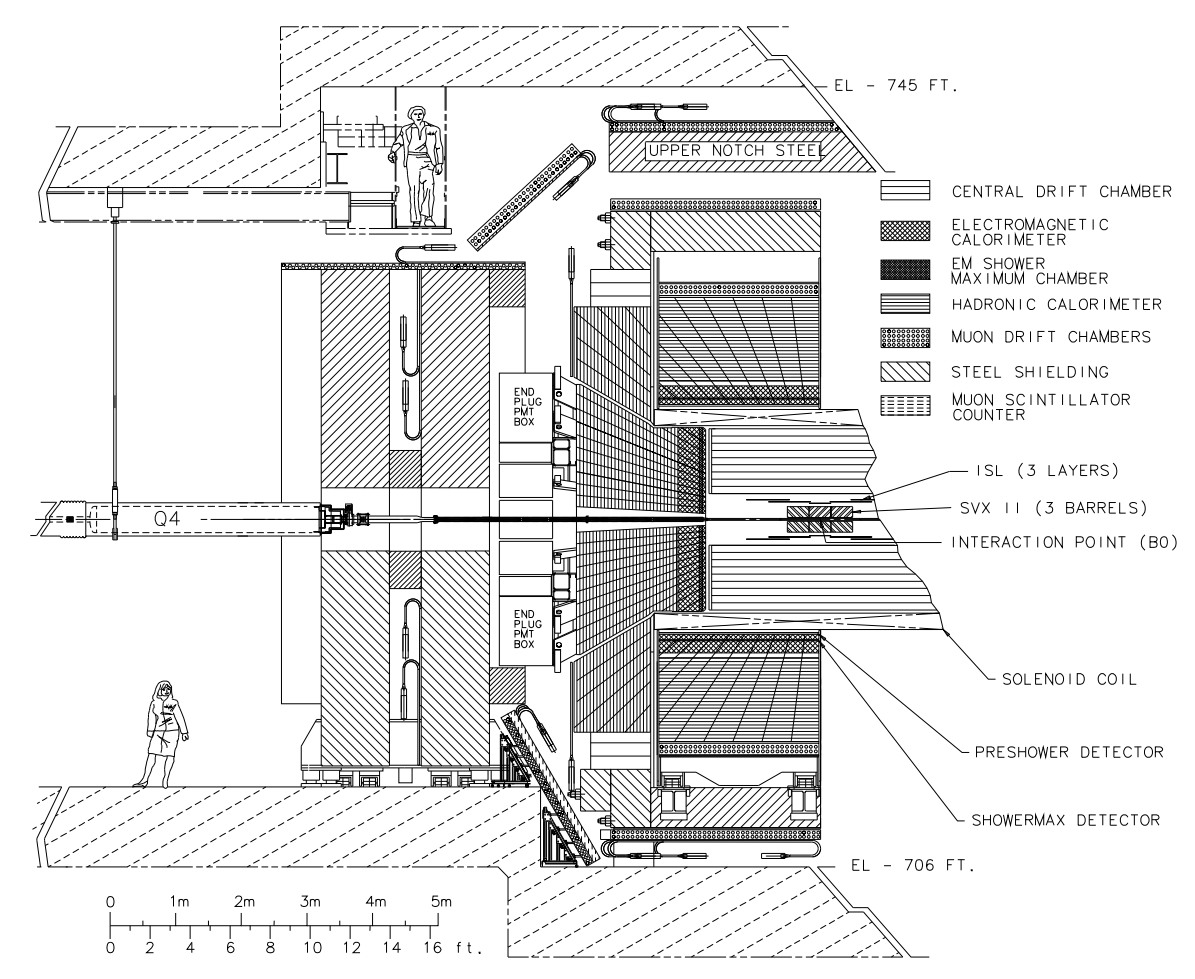

Figure 2.5: $r-\phi$ view of the CDF II detector (half detector shown).

\subsubsection{The tracking system}

The detector subsystems enclosed in a superconducting solenoid which generates a uniform axial magnetic field ${ }^{8}$ of $1.5 \mathrm{~T}$ are essentially dedicated to the reconstruction of the charged particles trajectories. The description of the each component of the tracking system is arranged according to an 'outside-in' scheme.

\section{The Central Outer Tracker}

The Central Outer Tracker (COT) [3] is a new open-cell drift chamber, replacing the Run I Central Tracking Chamber (CTC), that was unable to meet the requirements of the typical Run II environment, where high occupancies and event rates were expected.

From the mechanical point of view, the problem of occupancy has been solved by reducing the physical dimensions of the cells; this has been done by increasing by a factor four the number of available cells. Each cell, containing an array of 12 sense wires, is tilted with respect to the radial direction (see fig. 2.6) by an angle which minimizes the drift time, which in the COT is $\sim 5$ times shorter than in the CTC; this result, obtained also by optimizing the gas mixture contained by the chamber volume, meets the necessity of completing the collection of showering electrons before the next bunch crossing.

The overall cell layout follows from the Run I configuration: cells are organized in eight superlayers, whose arrangement is visible in fig. 2.6; even-numbered superlayers, equipped with axial wires, provide $r-\phi$ information, while $r-z$ information comes from the oddnumbered superlayers, whose wires are assembled with a small stereo angle $\left( \pm 3^{\circ}\right)$. Since

\footnotetext{
${ }^{8}$ The magnetic field produced by the CDF solenoid is parallel to the beamline; its uniformity refers to a cylindrical fiducial volume $\sim 3.5 \mathrm{~m}$ long and $\sim 2.8 \mathrm{~m}$ wide.
} 


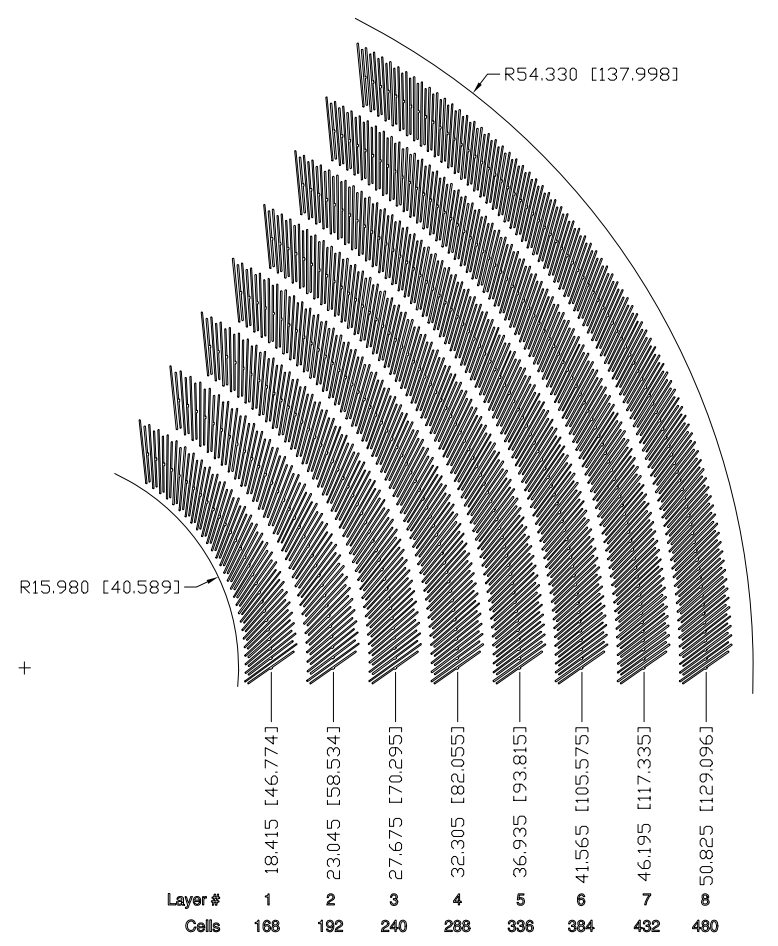

Figure 2.6: Layout of wire planes on a COT endplate.

all cells contain 12 sense wires, stereo sampling in the COT is double that in the Run I CTC.

The COT, covering a radial region between 40 and $138 \mathrm{~cm}$, is capable of tracking in the region with $|\eta| \lesssim 1$, provided that the track traversing its volume has $p_{T} \gtrsim 300 \mathrm{MeV} / \mathrm{c}$.

\section{The Intermediate Silicon Layers}

The Intermediate Silicon Layers (ISL) [4] detector is based on double-sided silicon crystals: one side of each crystal provides an axial (i.e. pure $r-\phi$ ) measurement, while the other one has stereo microstrips supplying $z$ information.

The structure of this detector varies according to the $\eta$ range:

$|\eta|<1$ : a single layer of silicon crystals is placed at an average radius of $\sim 22 \mathrm{~cm}$;

$1<|\eta|<2$ : two layers of silicon crystals are placed at average radii of $\sim 20$ and $\sim 29 \mathrm{~cm}$.

The number of layers in the ISL ranges between one and two according to $\eta$ so as to accommodate the possibility of stand-alone silicon tracking (in combination with SVX II see next section and fig. 2.10) not only in the central region but also where COT information is incomplete or missing; in both cases, the contribution of ISL, thanks to its stereo sampling, enables a full three-dimensional reconstruction. 


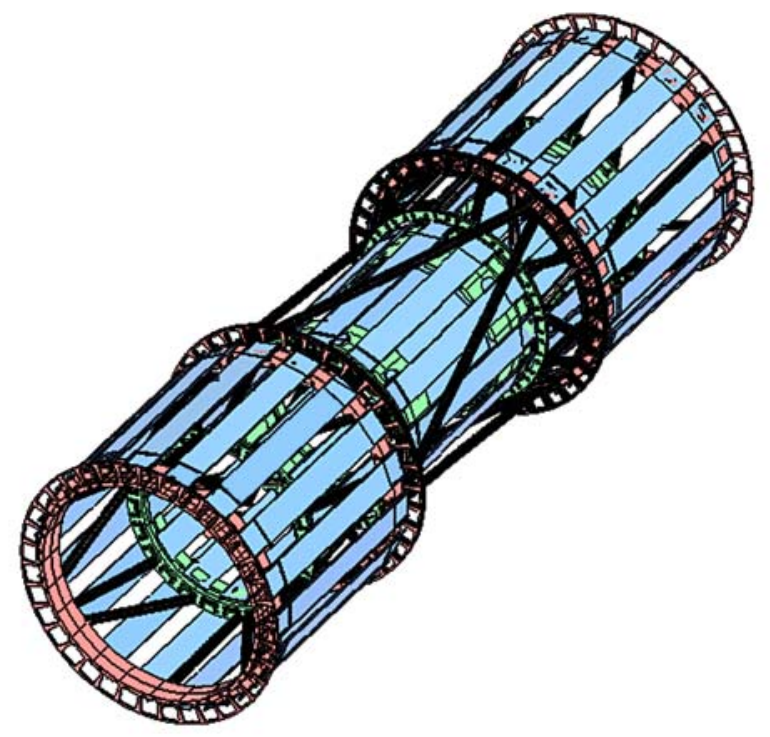

Figure 2.7: Perspective view of ISL.

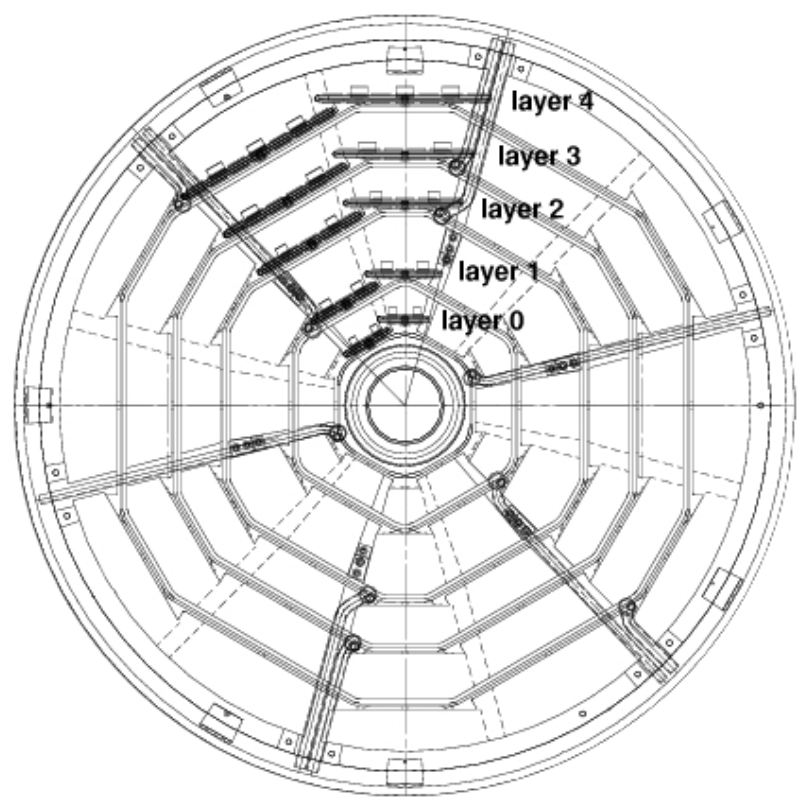

Figure 2.8: $r-\phi$ view of SVX II. 


\section{The Silicon Vertex Detector}

The Silicon Vertex Detector (SvX II) [5] is a five-layer detector wrapped around the beam pipe: layers, grouped into $12 \phi$-wedges, are placed at radii ranging from 2.5 and $17.3 \mathrm{~cm}$ (see fig. 2.8); each layer is assembled with double-sided silicon wafers. The detector is subdivided longitudinally into three identical barrels, which are aligned along the beamline, in order to provide adequate $z$ coverage $( \pm 45 \mathrm{~cm}$ along the $z$-direction from the nominal interaction point, $|\eta| \leq 2$ ).

One side of all layers is characterized by axial microstrips, providing $r-\phi$ information; the other side of the layers supplies $r-z$ information by means of stereo microstrips. Microstrips belonging to layers ${ }^{9} 0,1$ and 3 have a $90^{\circ}$ stereo angle; this angle decreases to $1.2^{\circ}$ for the remaining layers.

Svx II provides for high precision tracking and secondary vertex detection. In addition it supplies information to a dedicated trigger system (SVT - see $\S 3.1 .2$ ), that performs a fast search for displaced tracks (i.e. tracks not originated in the primary vertex).

\section{$\underline{\text { Layer } 00}$}

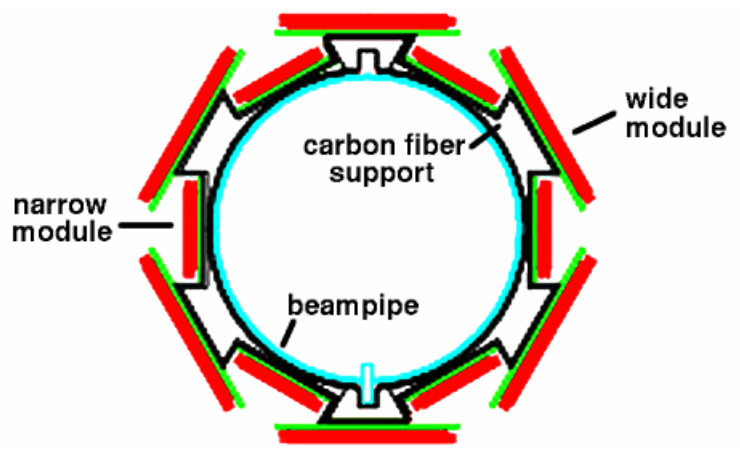

Figure 2.9: $r-\phi$ view of LAYER 00.

Layer 00 [6] (see Fig.2.9) is a single-sided layer of silicon crystals $(|\eta| \leq 4)$ placed on the outer side of the beam pipe at a radial distance of $\sim 1.5 \mathrm{~cm}$ from the beamline (see Fig. 2.10); it supplies $r-\phi$ information only, but its position makes of it the perfect tool for increasing the track impact parameter resolution of the tracking system.

\subsubsection{The time of flight}

Placed in the gap between the magnet and the outer wall of the COT, the detector for time of flight measurement [7][8] is essentially an array of scintillator bars $\sim 3 \mathrm{~m}$ long and $\sim 4 \mathrm{~cm}$ thick; their section is trapezoidal in shape, in order to better fit the cylindrical cavity they are supposed to fill, at the same time reducing the uninstrumented regions (cracks). The average width for maximal coverage turned out to be $\sim 4 \mathrm{~cm}$.

\footnotetext{
${ }^{9}$ Layers are numbered from 0 to 4 according to an 'inside-out' order.
} 


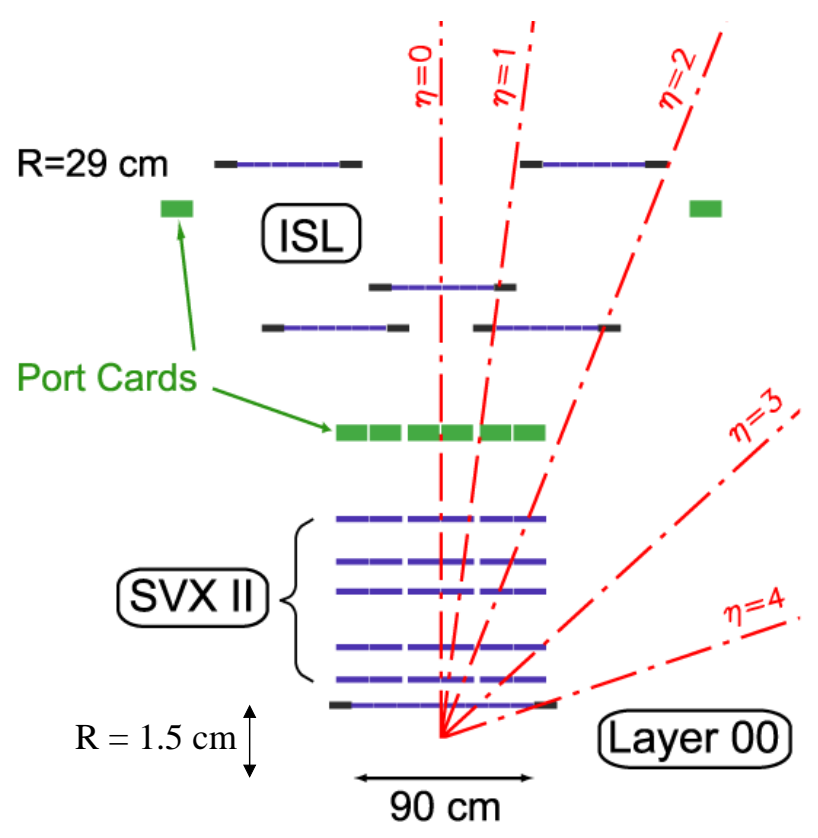

Figure 2.10: $r-z$ view of silicon detectors.

Scintillator bars are read at both ends by photomultiplier tubes, both providing a leading-edge time and a pulse-height. From a comparison of the readings performed at each end one can also estimate the $z$-coordinate of the charged particle that traversed the scintillating material, which is then used for matching the time of flight measurement to a track reconstructed by the tracking system. The time of flight measurement is performed computing the time interval between the interaction and the mean time of the signal in the scintillators. This measurement is motivated principally by the need to identify low-energy particles.

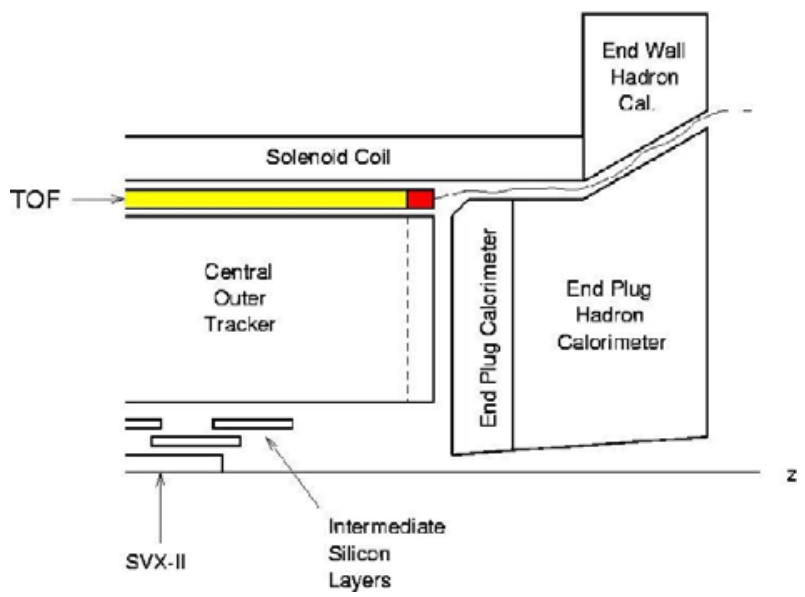

Figure 2.11: TOF position. 


\subsubsection{The calorimeters}

The CDF calorimetry system [9][10] consists of inner electromagnetic and outer hadronic sections, both segmented according to a common scheme of azimuthal wedges $(\Delta \phi=$ $15^{\circ}$ ), each wedge corresponding to an array of towers projecting towards the geometrical center of the detector. The result is a cylindrical barrel providing almost full azimuthal acceptance for $|\eta|<3.64$ coverage.

The purpose of calorimeters is to measure the energy ${ }^{10}$ depositions of charged and neutral particles leaving the tracking and magnet regions, as well as providing information about the missing transverse energy; as will be explained in the next chapter, this detector is of crucial importance also for cluster and jet reconstruction.

\section{The central and endwall calorimeters}

The central and endwall calorimeters which cover the range $|\eta|<1.1(|\eta|<1.2$ for hadronic section), have been retained almost unchanged from Run I, the only major upgrades being related to readout electronics. Each tower, corresponding to $\Delta \eta \times \Delta \phi=0.1 \times 15^{\circ}$, is a an independent sampling system consisting of an electromagnetic section of alternating layers of lead and scintillator followed by an iron-scintillator sandwich hadronic section. The former, contributing a total thickness of $\sim 18$ radiation lengths $\left(X_{0}\right)$, is dedicated to the electromagnetic energy component, while the latter, corresponding to $\sim 5$ absorption lengths $\left(\lambda_{0}\right)$, provides a measure of the hadronic energy.

Light pulses recorded in the various scintillator tiles are collected by wave length shifters and carried outside the detector volume by light guides, where they are read by photomultiplier tubes.

A perspective view of a central calorimeter half-wedge ${ }^{11}$ with $\eta>0$ is depicted in fig. 2.12, where both the arrangement in projective towers and the light-gathering system (only the electromagnetic system is sketched) are visible.

Energy resolutions achievable by this detector are influenced by their mechanical structure (sampling) and by stochastic fluctuations due to the photomultipliers response; global estimates assign resolutions of $14 \% / \sqrt{E_{T}[\mathrm{GeV}]}$ for electromagnetic and $75 \% / \sqrt{E_{T}[\mathrm{GeV}]}$ for hadronic towers.

Two position detectors are included in each wedge of the central electromagnetic calorimeter:

$\diamond$ The Central Electromagnetic Strip chamber (CES). Embedded within the lead-scintillator sandwich at a radial depth of $r=184 \mathrm{~cm}^{12}$, in correspondence to maximum shower development, two-dimensional strip-wire chambers [11] provide position and pulse-height information about electromagnetic showers by measuring their charge deposition. Chamber geometry is usually described in terms of the local wedge-coordinate system defined in fig. 2.12: each half-wedge hosts two CES

\footnotetext{
${ }^{10}$ Although calorimetry measurements provide estimates of $E$ (the total energy deposited) only transverse energy components $\left(E_{T}\right)$ are relevant at hadron colliders. This is because the total energy of hard scattering processes emerging from $p \bar{p}$ collisions varies with the Bjorken $x$ of each colliding parton.

${ }^{11} \mathrm{~A}$ half-wedge subtends a $\Delta \phi=15^{\circ}, \eta \gtrless 0$ region.

${ }^{12}$ Equivalent to $\sim 5.9 X_{0}$ (including the coil, which contributes by $\sim 1 X_{0}$ ).
} 


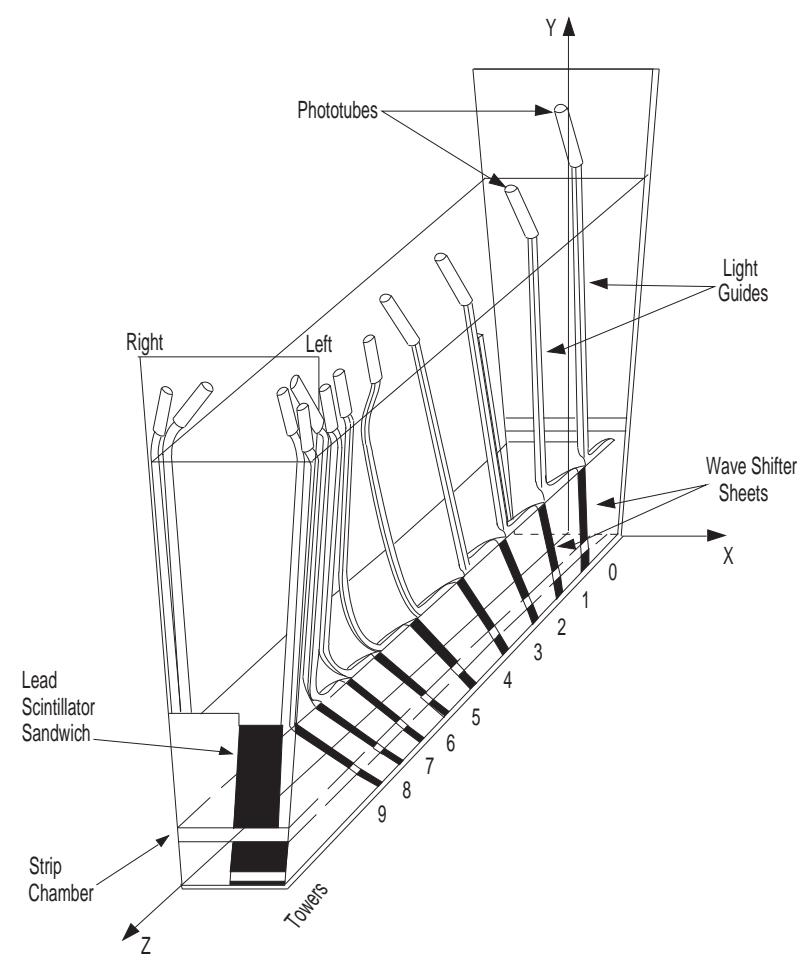

Figure 2.12: Perspective view of central calorimeter half-wedge.

modules, providing coverage in the $z=6.2 \div 121.2 \mathrm{~cm}$ and $z=121.2 \div 239.6 \mathrm{~cm}$ regions; chambers are segmented along the $z$ direction in strips having an approximate pitch of $2 \mathrm{~cm}$, leading to a total of 64 strip channels in each chamber module. A 32wire array is coupled to each chamber module; wires, with a $\sim 2.5 \mathrm{~cm}$ spacing, are parallel to the $z$ direction, providing in this way a $90^{\circ}$ reading of the charge deposition with respect to strips. The CES can contribute effectively to increase the purity of electromagnetic objects: besides providing a finer azimuthal segmentation than calorimeter towers, CES can reject early hadronic showers occurring in the outer portion of the electromagnetic towers.

$\diamond$ The Central Pre-Radiator chamber ( $\mathrm{CPR})$. Mounted in front of the innermost face of the electromagnetic towers, the preradiator consists of two wire chamber modules for each half-wedge; in practice, this device follows the same structure and wire arrangement as the CES wire chamber, the only differences being related to shorter wire spacing $(\sim 2.2 \mathrm{~cm})$ to fit the projecting wedge profile and the slightly reduced $\eta$-acceptance $(z=7.14 \div 119.7 \mathrm{~cm}$ and $z=123.5 \div 235.76 \mathrm{~cm}$ delimit the boundaries of active regions). The preradiator collects the charge depositions released by showers that initiate in the tracking or coil material; the information provided by this detector can be used for further background rejection in selecting electrons or photons (which can be better separated from pions because of their average larger deposition in the $\mathrm{CPR}$ ).

\section{The plug calorimeters}




\begin{tabular}{|l|l|}
\hline$|\eta|$ range & $\Delta \phi \times \Delta \eta$ \\
\hline $0.0-1.1$ & $15^{\circ} \times 0.1$ \\
$1.1-1.8$ & $7.5^{\circ} \times 0.1$ \\
$1.8-2.1$ & $7.5^{\circ} \times 0.16$ \\
$2.1-3.64$ & $15^{\circ} \times(0.2-0.64)$ \\
\hline
\end{tabular}

Table 2.1: Calorimeter $\Delta \phi \times \Delta \eta$ segmentations.

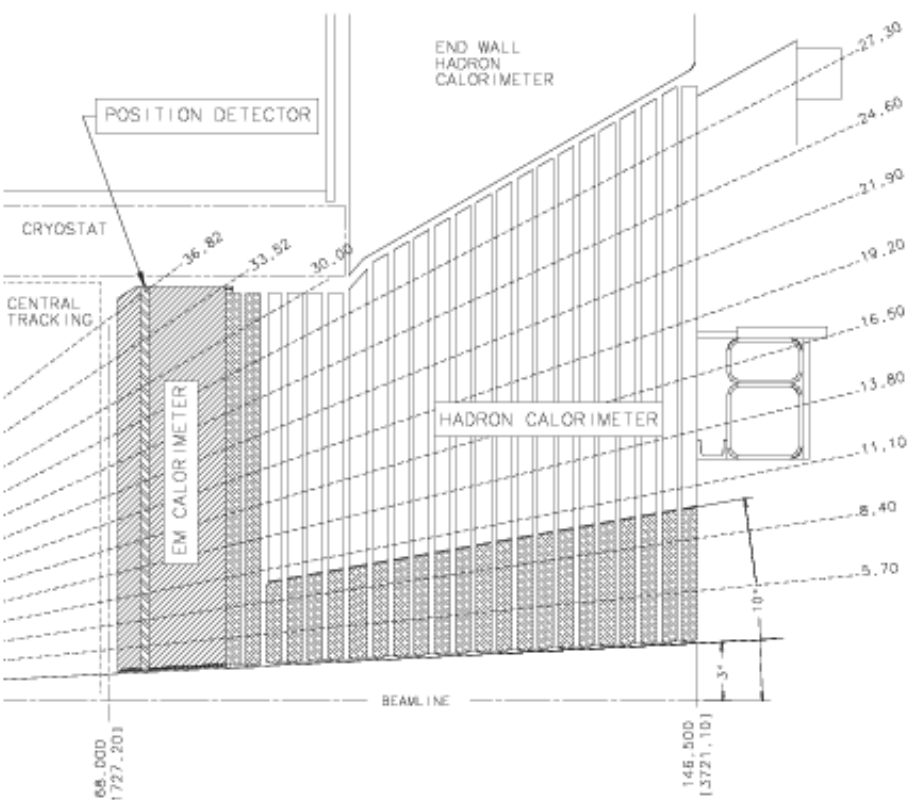

Figure 2.13: r-z view of plug calorimeter system.

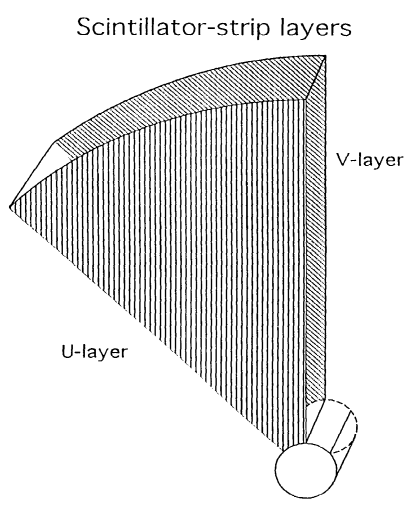

Figure 2.14: Perspective view of plug shower maximum detector.

The active material for the Run I plug calorimeters consisted of proportional tubes, whose time response was too slow for operational conditions of Run II; for this reason, this detector was substituted by new units, whose $r-z$ section is depicted in fig. 2.13, which also shows its relative position within CDF. From a functional point of view, the upgraded plug calorimeters essentially follow the scheme of the central calorimeter, with both the electromagnetic and hadronic sections relying on alternating layers of absorbing material (lead and iron respectively) and scintillator tiles, leading to a thickness of $\sim 21 X_{0}\left(\sim 1 \lambda_{0}\right)$ and $\sim 7 \lambda_{0}$ in the two cases. As for the central calorimeter, scintillators are read out by photomultiplier tubes (placed on the outside of each end-plug) receiving the light pulses through bundles of wave length shifters, which collect the signals from the tiles, and light guides.

The upgraded plug calorimeters cover the region $1.10<|\eta|<3.64$. While the central calorimeter is characterized by almost constant $\eta-\phi$ granularity, both $\eta$ and $\phi$ segmentation of the plug decrease with increasing $\eta ; \Delta \eta$ ranges from 0.1 to 0.64 on approaching the beamline and the azimuthal segmentation decreases from $7.5^{\circ}$ to $15^{\circ}$ at the boundary between the fifth and the fourth highest $\eta$ towers (see Tab. 2.1). 
The energy resolutions are the result of limited sampling performed by scintillating tiles and stochastic fluctuations affecting the photomultipliers. Electromagnetic and hadronic resolutions have been measured for single electrons and pions and found to be $16 \% / \sqrt{E[\mathrm{GeV}]}$ and $80 \div 90 \% / \sqrt{E[\mathrm{GeV}]}$ respectively.

Position detectors are included in each wedge of the calorimeter:

$\diamond$ The Plug Electromagnetic ShowerMax detector (PES). The shower maximum detector [12] is embedded at a radial depth of $\sim 6 X_{0}$. In this case, instead of relying on the wire-strip chamber technique used for the central calorimeter, arrays of scintillating strips have been chosen. The PES is divided in eight $45^{\circ}$ azimuthal sectors $^{13}$, each consisting of two layers (named $\mathrm{U}$ and $\mathrm{V}$ ) of $5 \mathrm{~mm}$ pitch scintillating strips; strips belonging to the same layer are oriented along a common direction parallel with one of the two sector radial boundaries (see fig. 2.14), in such a way that $\mathrm{U}$ and $\mathrm{V}$ strips tend an angle of $45^{\circ}$ to each other, which enables two-dimensional position measurements.

Light signals in this detector are collected by wave length shifter fibers embedded in the strips and read by means of photomultiplier tubes placed at the rear of the plug calorimeter.

$\diamond$ The Plug Pre-Radiator detector (PPR). The plug preradiator [13], as does the CPR, enhances discrimination between electrons and pions. It constitutes in the first scintillator layer of the plug calorimeter following the same layout.

\subsubsection{The muon system}

Muon detectors [2][14] are arranged in such a way as to enclose the whole CDF detector (see fig. 2.4); muon detectors are the outermost detectors because they exploit the fact that muons are very penetrating particles so that they are ranged out from other charged tracks by inner detector material and shielding.

The CDF muon system has undergone two substantial upgrades for Run II: first the coverage of previously uninstrumented central regions and second an extension of acceptance to higher $\eta$.

The acceptance of the Run II muon system is illustrated in Fig. 2.15. The muon system consists of four subdetectors described in the following.

The Central Muon Chambers (CMU), placed immediately outside the hadron calorimeter, remained unchanged from Run I: they consist of 144 modules each containing $166.35 \times 2.68 \mathrm{~cm}$ rectangular drift cells stacked in four layers with a small azimuthal offset; three such modules cover a half-wedge of the calorimeter, providing a coverage for $|\eta| \lesssim 0.6$. Muons with $p_{T} \gtrsim 1.5 \mathrm{GeV} / \mathrm{c}$ are able to traverse the whole calorimeter region and leave a signal in the CMU; their position can be determined by merging the information coming from drift times (supplying $\phi$ ) with a $z$ coordinate estimated on the basis of charge division. Drift tubes are driven in proportional mode with a maximum drift time of $800 \mathrm{~ns}$.

The Central Muon Upgrade (CMP) consists of a second set of four staggered layers of $2.5 \times 15 \mathrm{~cm}$ drift tubes arranged behind an additional $60 \mathrm{~cm}$ of steel ${ }^{14}$ according to a

\footnotetext{
${ }^{13}$ Sectors segmentation are matched to tower boundaries.

${ }^{14}$ The magnetic field return yoke.
} 


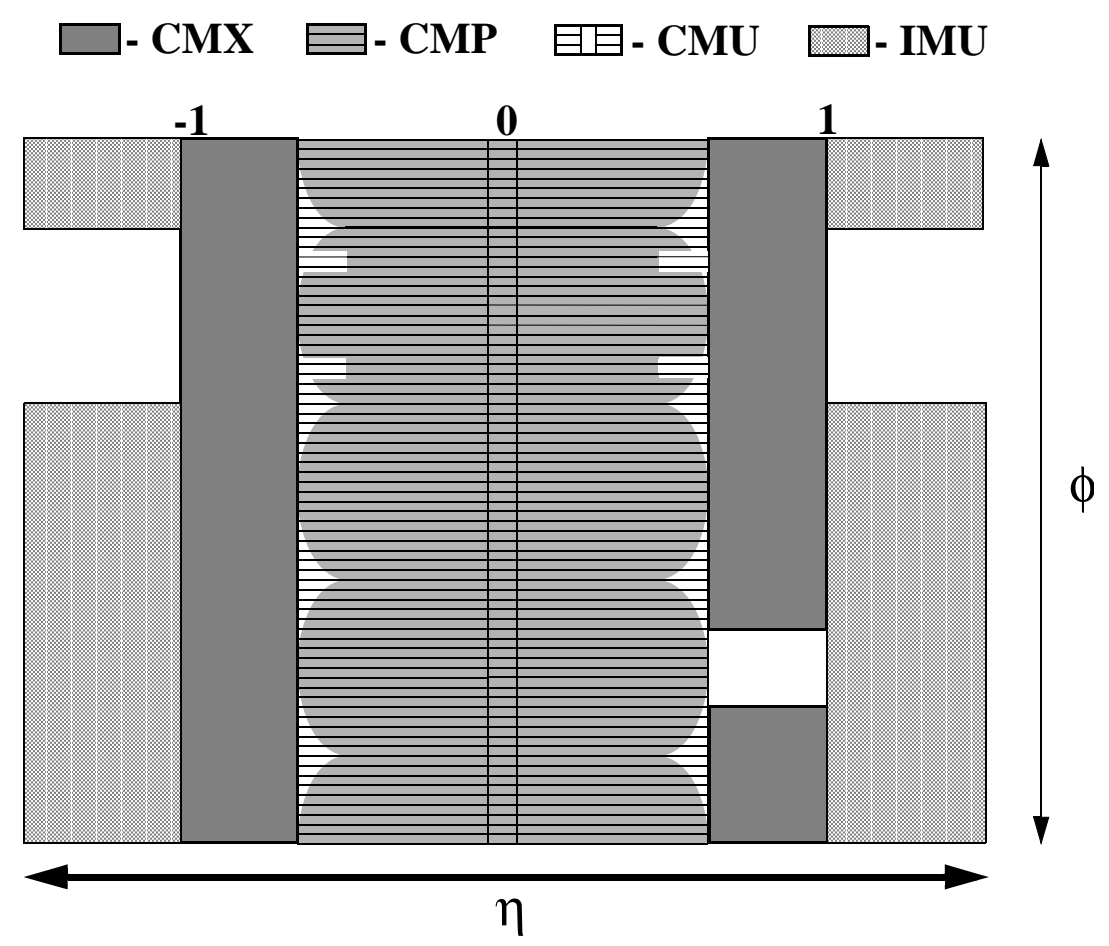

Figure 2.15: Muon system $\eta-\phi$ map.

rectangular geometry (i.e. they form a box around the central detector); the mismatch between the inner cylindrical structure and the outer box results in a curved acceptance boundary, which can be seen in fig. 2.15. Muons must have $p_{T} \gtrsim 2.2 \mathrm{GeV} / \mathrm{c}$ to reach the CMP. As for the CMU, drift tubes run in proportional mode (maximum drift time of $1.4 \mu \mathrm{s})$, but they do not provide $z$ information.

On the outer surface of the CMP a layer of rectangular scintillator counters ${ }^{15}$ is installed in such a way that each scintillator tile covers two drift cells in width and half a cell in length. CMP acceptance has been increased by $17 \%$ for Run II.

The Central Muon Extension (CMx) provides coverage in the $0.6 \lesssim|\eta| \lesssim 1$ by means of arrays of drift tubes arranged in conical sections, which are then positioned at each end of the central detector; CMX array geometry follows the CMP scheme. Drift tubes are embedded within two layers of scintillator counters ${ }^{16}$; four scintillator tiles cover a $\Delta \phi=15^{\circ}$ range, with tiles being staggered between the inner and the outer layers. The drift tubes, which are reached by muons with $p_{T} \gtrsim 1.4 \mathrm{GeV} / \mathrm{c}$, are operated in proportional mode with maximum drift time of $1.4 \mu$ s and provide $\phi$ information; $z$ information, in the other hand, is obtained from scintillator timing. CMX will benefit an improved acceptance by $45 \%$ in Run II.

The Intermediate Muon Detectors (IMU) extends muon acceptance to $|\eta| \leq 1.5$. Placed on the outer surface of each toroid, its main structure consists of a barrel of drift chambers (run in proportional mode with a maximum drift time of $800 \mathrm{~ns}$ ) coupled with scintillator tiles; the arrangement follows the CMP/CSP scheme. These detectors are reachable by muons with $p_{T} \gtrsim 1.4 \div 2.0 \mathrm{GeV} / \mathrm{c}$.

The slow response of the muon system can be overcome by exploiting their high

\footnotetext{
${ }^{15}$ These form the Central Scintillator Upgrade, or CSP.

${ }^{16}$ Central Scintillator Extension or CSX.
} 
granularity, which, in principle, enables integration of signals collected by muon chambers over several beam crosses; this, however, is feasible only as long as detector occupancy is relatively low. During Run I, it was observed that most ( $\gtrsim 95 \%)$ of the ionization detected in the muon system originated from the Main Ring rather than from $p \bar{p}$ collisions and TEVATRON beam halo. In Run II, this situation is much improved, because the Main Ring has been substituted by the Main Injector and because additional shielding has been installed. This additional shielding protects the most exposed devices (CMX and CMP), and therefore reduces the occupancy of muon detectors. These improvements, together with the detector upgrades described above, enhanced the performances of the muon system.

\subsubsection{The Cherenkov luminosity counters}

In order to measure the luminosity it is necessary to count the number of $p \bar{p}$ interactions per bunch crossing. This operation is performed by means of two Cherenkov Luminosity counters (CLC) [15][16]. They are located inside the endplug calorimeters, in the forward and backward regions $(3.7<|\eta|<4.7)$. Each module consists of 48 thin, long, conical, gas filled Cherenkov counters. These are arranged around the beam pipe in three concentric layers with 16 counters each and pointing to the interaction point. Prompt particles coming from the $p \bar{p}$ collisions traverse the full length of the counter and generate a large amplitude signal in the photomultipliers. While, particles originating from beam halo or from secondary interactions of prompt particles are softer: they traverse the counters at large angles with shorter path lenghts and their light suffers a large number of reflections, giving thus a much smaller signal than prompt particles. Luminosity is defined by considering time-coincidences between signals from prompt particles (both in $p$ and $\bar{p}$ directions) leaving the interaction point.

\subsubsection{The trigger system}

The upgrated characteristics of the detector, which, as described above, were needed by new running conditions of the TEVATRON, require the data acquisition (DAQ) and the trigger system to undergo corresponding upgrades.

As previously mentioned, the major change affecting data acquisition in Run II it the reduced time between beam crossings. During Run I the time between two beam crossings $(3.5 \mu \mathrm{s})$ was long enough to permit processing of the calorimeter signals and reach a Level 1 trigger decision before the occurrence of the next event. In Run II, where the bunch spacing is reduced to $396 \mathrm{~ns}$ this is not be possible. Moreover, the increased instantaneous luminosity regime requires more sophisticated trigger primitives to provide effective rate reduction. The only solution compatible with the new operational conditions consists in storing every incoming event in a memory buffer, using the time recovered from fast rejects to provide adequate processing of the remaining events. Because the Run I CDF configuration could not match the new operational conditions, the whole trigger and DAQ systems had to be replaced for Run II.

A simplified picture of the three-level architecture of the CDF trigger system data flow is shown in fig. 2.16. A Level 1 latency time of $5.5 \mu \mathrm{s}$ was chosen to allow readout, data transmission and trigger processing; the size of this time interval defines the dimension

of the memory buffers, which are located directly on the readout boards of the detector 


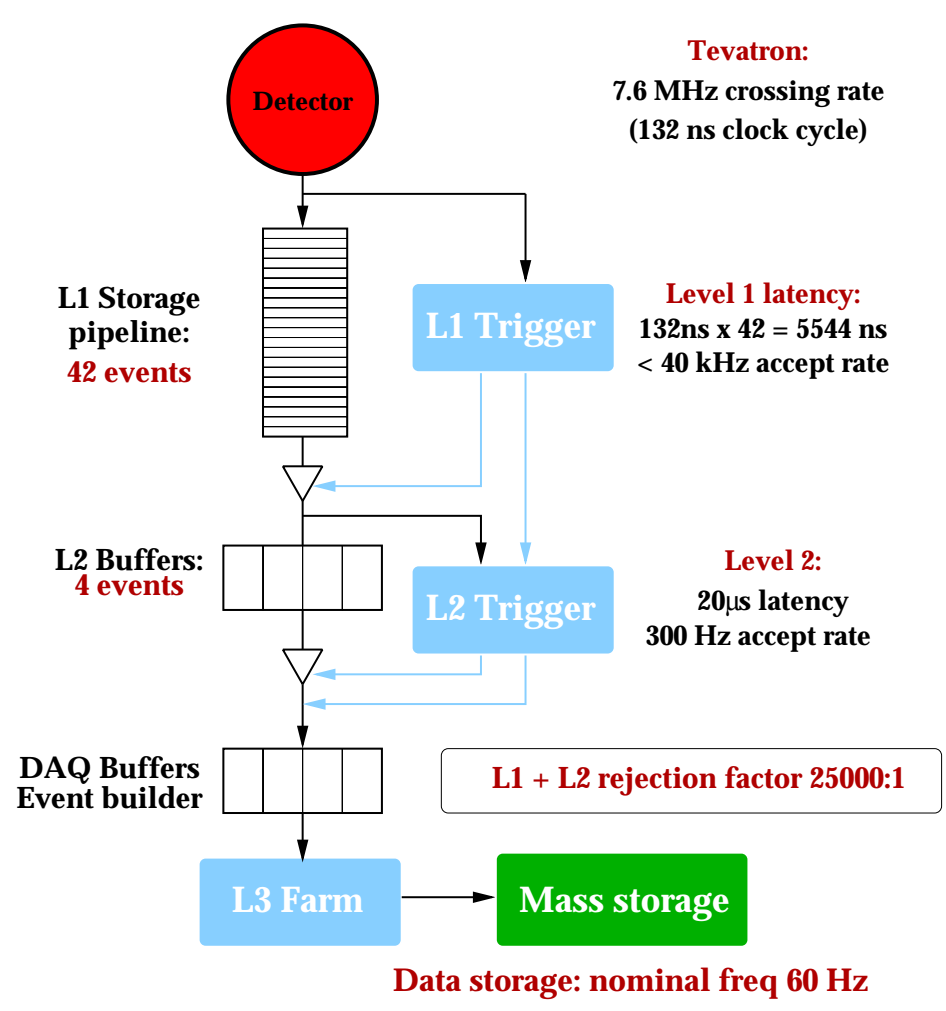

Figure 2.16: Buffering and pipelining in the Run II CDF trigger system.

subsystems in order to save time: Assuming a beam spacing of $132 \mathrm{~ns}^{17}$, a 42 -event memory stack is required. Events passing the Level 1 trigger requirements are then pipelined into one of four onboard Level 2 buffers $^{18}$; the average time expected for processing one event sets the Level 1 trigger bandwidth, which is the maximum rate of events that can be accepted. In order to keep dead-time within acceptable values $(\lesssim 10 \%)$, each separate Level 2 buffer is connected to a two-step pipeline, each step having a latency time of $10 \mu \mathrm{s}$ [17]:

step 1: single detectors responses are analyzed;

step 2: the combination of the outcome of step 1 are merged and trigger decisions are cast on a whole-event basis.

Events passing Level 2 are transmitted to a PC farm, where events are fully reconstructed; this happens at Level 3, after which, if an event is accepted, it is permanently stored.

\footnotetext{
${ }^{17}$ Provision was originally made for $132 \mathrm{~ns}$ bunch intervals. This condition was never realized.

${ }^{18}$ Note that at Level 2 processing is an asynchronous process, as opposed to Level 1, where events are analyzed in the same order as they occurred.
} 


\section{Bibliography}

[1] Refer to web page http://beamdocs.fnal.gov/cgi-bin/public/DocDB/ListTopics.

[2] F. Abe et Al., (The CDF II Collaboration), The CDF II Detector Technical Design Report, FERMILAB-PUB-96-390-E, Oct 1996.

[3] T. Affolder et Al., COT Central Outer Tracker, Nucl. Instrum. Meth. A526:249, 2004.

[4] A. Affolder et Al., ISL: Intermediate Silicon Layers Detector for the CDF Experiment, Nucl. Instrum. Meth. A453:84-88, 2000.

[5] A. Sill, SVX-II: CDF Run II Silicon Tracking Projects, Nucl. Instrum. Meth. A447:18, 2000 .

[6] C. Hill, L00: Operational Experience and Performance of the CDFII Silicon Detector, FERMILAB-CONF-03-412-E, Jan 2004. 9pp., Nucl. Instrum. Meth. A530:1-6, 2004.

[7] The CDF Collaboration, A Time of Flight System for CDF, CDF internal note 2573, Feb 1994.

[8] D. Acosta at Al., A Time-Of-Flight Detector in CDF-II, Nucl. Instrum. Meth. A518:605-608, 2004.

[9] S. Kuhlmann et Al., The CDF Calorimetry Upgrade for Run IIb, Nucl. Instrum. Meth. A518:39-41, 2004.

[10] R. Erbacher, Calorimetry in CDF Run 2, FERMILAB-CONF-02/251-E, 2002.

[11] A. Solodsky, The Shower-Maximum Detector for CDF II, FERMILAB-CONF98/085-E, 1998.

[12] G. Apollinari at Al., Shower Maximum Detector for the CDF Plug Upgrade Calorimeter, Nucl. Instrum. Meth. A412:515-526, 1998.

[13] M. Albrow et Al., A preshower detector for the CDF Plug Upgrade: test beam results Nucl. Instrum. Meth. A431:101-111, 1999.

[14] L. Cerrito et Al., Performance of the CMU and CMP Muon Chambers, CDF internal note $6055,2002$.

[15] D. Acosta et Al., The CDF Luminosity Monitor, Nucl. Instrum. Meth. A461:540-544, 2001. 
[16] D. Acosta et Al., The Performance of the CDF Luminosity Monitor, Nucl. Instrum. Meth. A494:57-62, 2002.

[17] H. Frisch et Al., Conceptual Design of a Deadtimeless Trigger for the CDF Trigger Upgrade, CDF internal note 2038, Dec 1994. 


\section{Chapter 3}

\section{The CDF trigger}

At $\sqrt{s} \sim 2 \mathrm{TeV}$, the inelastic cross section for $p \bar{p}$ scattering is $\sim 50 \mathrm{mb}$, which corresponds to 7.6 million collisions per second for a typical Run II instantaneous luminosity of $10^{32} \mathrm{~cm}^{-2} \mathrm{~s}^{-1}$. This value must be compared with the typical rate for processes of particular interest at hadron colliders; $8 \times 10^{-4} \mathrm{~Hz}$ for top (corresponding to $\sigma \sim 6 \mathrm{pb}$ for $M_{\mathrm{t}} \sim 175 \mathrm{GeV} / \mathrm{c}^{2}$ ) and $3 \times 10^{-5} \mathrm{~Hz}$ for (SM) Higgs production (corresponding to $\sigma \sim 0.26 \mathrm{pb}$ for $M_{\mathrm{H}} \sim 120 \mathrm{GeV} / \mathrm{c}^{2}$ ) respectively.

The relative abundances of $p \bar{p}$ collisions require that data-taking at a hadron collider detector be controlled by a mechanism that filters out a very large majority of the events which do not have the characteristic signatures of the physical processes one is interested in. This mechanism is known as trigger system. It essentially consists of a collection of specific hardware modules driven by speed-optimized software capable of performing a selection on the basis of pattern recognition and reconstruction. Events which are selected by the trigger system are saved permanently on mass storage device and subsequently fully reconstructed offline.

A description of each level of the CDF trigger is given in this chapter. with emphasis on the Level 1 and 2. Level 3 data processing is based on the offline reconstruction software which is illustrated in the next chapter. In the last section a description of the trigger of interest for this thesis is given.

\subsection{Trigger primitives}

The CDF trigger system [1] is organized according to a three-level architecture (see Fig. 3.1); each level output is fed as input into the next level. The underlying philosophy of this procedure consists essentially in reducing the rate at lower levels by means of very conservative requirements aimed mainly at reducing the dead time, allowing in this way more sophisticated selections to be performed at higher levels.

At each level, a set of primitives, essentially physics objects directly measured by the detector at lower levels (such as energy depositions in the calorimeter) or obtained from them by running some algorithm at higher levels, are defined. Depending on the signal one wants to isolate, specific requirements are applied to a subset of the primitives available at a given level; this defines a specific trigger for that level.

Once triggers are built for each level, links across different levels are established by defining trigger paths; a trigger path identifies a unique combination of a Level 1, a Level 2 and a Level 3 triggers; in other words, a trigger path establishes a logic AND between 


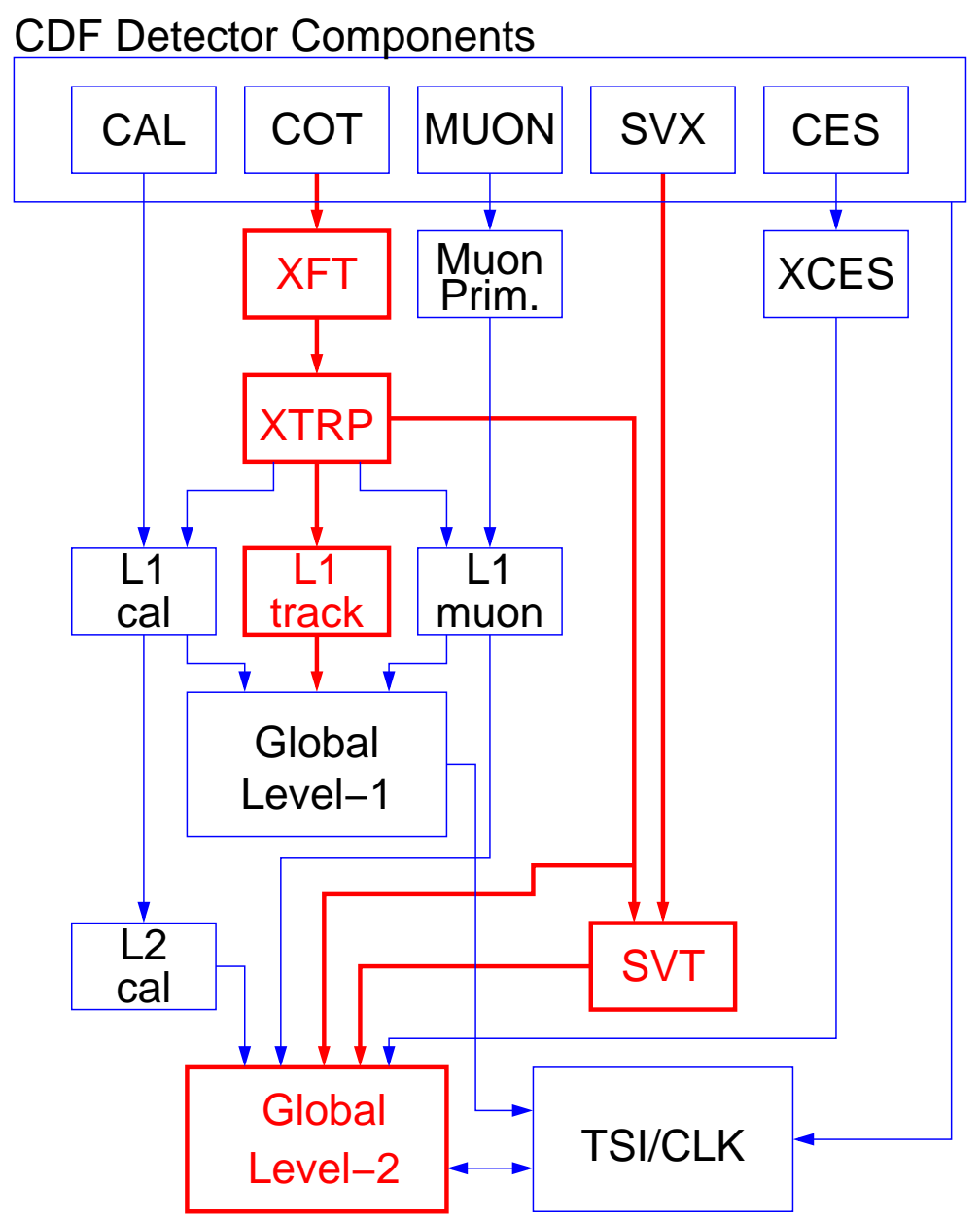

Figure 3.1: Schematic diagram of CDF trigger architecture. 


\begin{tabular}{||l||c|c||}
\hline & Run II Specifications & Run II Achieved \\
\hline \hline Level 1 bandwidth & $40 \mathrm{kHz}(400 \mu \mathrm{b})$ & $25 \mathrm{kHz}(250 \mu \mathrm{b})$ \\
Level 2 bandwidth & $300 \mathrm{~Hz}(3.0 \mu \mathrm{b})$ & $450 \mathrm{~Hz}(4.5 \mu \mathrm{b})$ \\
Level 3 bandwidth & $75 \mathrm{~Hz}(750 \mathrm{nb})$ & $100 \mathrm{~Hz}(1 \mu \mathrm{b})$ \\
\hline
\end{tabular}

Table 3.1: Run II bandwidths for an instataneous luminosity of $1 \times 10^{32} \mathrm{~cm}^{-2} \mathrm{~s}^{-1}$ : specifications [1] versus achieved values.

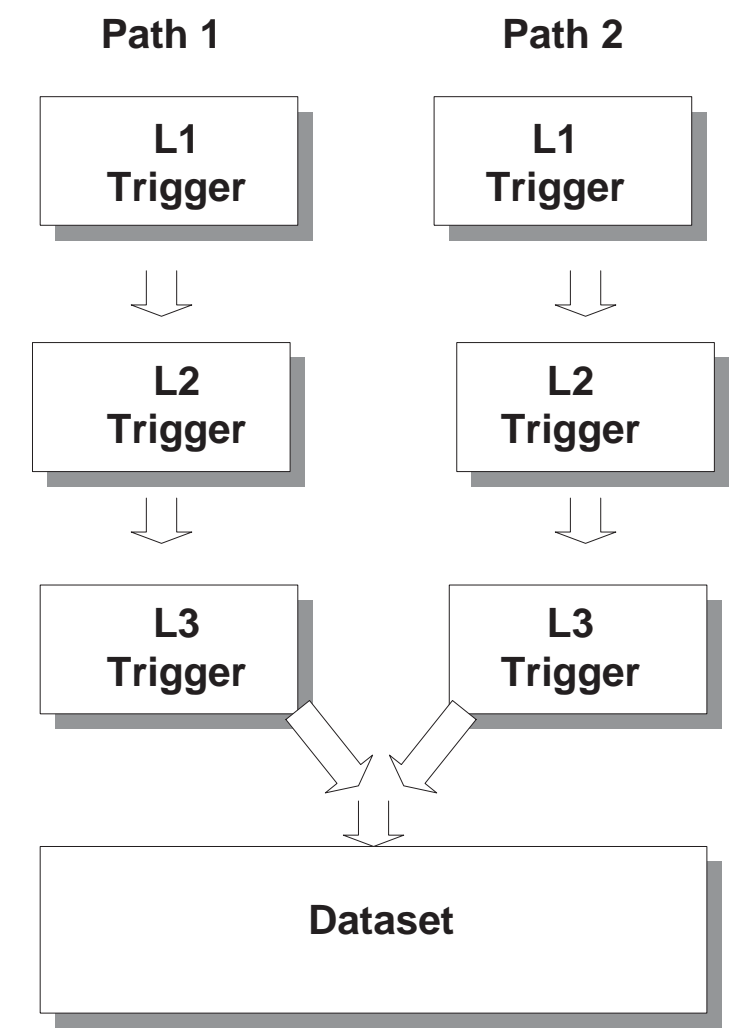

Figure 3.2: Schematic diagram of trigger primitives, paths and datasets.

selection procedures at different levels. Datasets (or data streams) are then formed by merging the data samples collected via different trigger paths; hence, datasets are defined by a logic OR between trigger paths (see Fig. 3.2).

The maximum allowed rate for a given level (which is often referred to as 'bandwidth') reflects the hardware's capability of buffering and, at Level 3, permanently storing the collected events in the unit time; table 3.1 summarizes bandwidth specifications compared to the achieved bandwidths in a typical Run II scenario. Since the $p \bar{p}$ collision rate increases with increasing luminosity, the trigger system needs to be constantly monitored to verify that the trigger requirements are compatible with the available rates.

In the following, a list of CDF trigger primitives is presented. 


\subsubsection{Level 1}

Level 1 trigger primitives have been constructed on the basis of very simple mechanisms, aimed at minimizing the dead time required for a decision: a raw reconstruction of physics objects is performed and a decision is taken by simply counting them. The nominal dead time is $\sim 5.5 \mu \mathrm{s}$. These primitives are completely hardware-based and can be divided into four categories according to the detector the primitives are based on.

\section{Calorimetry}

Calorimetry primitives can be subdivided into two classes:

- object primitives. Energy deposits detected ${ }^{1}$ in the central and plug calorimeter weighted by a $\sin \theta$ factor. At Level 1 , towers are not considered individually, but merged in pairs along $\eta$; the primitives so defined are addressed as 'trigger towers' and are $\Delta \eta \simeq 0.2 \times \Delta \phi=15^{\circ}$ wide $^{2}$; the result is a $24 \times 24 \eta-\phi$ map of the calorimeter region extending to $|\eta|<3.6$. Electromagnetic (EM) and total (EM+HAD) contributions are treated independently;

- global primitives. Transverse energy (EM+HAD) deposits recorded in all trigger towers above a pre-defined threshold (a $1 \mathrm{GeV}$ threshold has been set on the basis of efficiency maximization studies) are involved in two different sum procedures:

$\diamond$ all $\eta-\phi$ towers are summed together into the definition of $\Sigma E_{T}$;

$\diamond$ each set of 24 modules corresponding to a $\phi$-wedge are added together and the $\Sigma E_{T x}=\Sigma E_{T}(\phi) \cos \phi, \Sigma E_{T y}=\Sigma E_{T}(\phi) \sin \phi$ sums computed; from them, the missing transverse energy $\mathbb{E}_{T}$ is obtained (see Section 4.5 for the definition of this variable).

Correspondingly, object and global triggers are defined; in both cases the trigger requires a comparison between a detected transverse energy (single trigger tower $E_{T}$ and $E_{T}^{\text {had }} / E_{T}^{\text {em }}$, $\Sigma E_{T}$ and $\mathbb{E}_{T}$ ) and a threshold (EM and EM+HAD thresholds can be set independently). In the case of object triggers, the number of towers above threshold is counted; this is done by a 1 -bit sum $(0, \geqslant 1)$ for single-object triggers and by a 2 -bit $\operatorname{sum}(0,1,2, \geqslant 3)$ for di-object triggers.

\section{$\underline{\text { XFT }}$}

XFT is the acronym of eXtremely Fast Tracker and stands for a hardwired algorithm used for track finding in the COT. The guiding idea is performing a fast $r-\phi$ trackreconstruction, returning $p_{T}, \phi_{0}$ (the azimuthal direction of the track at the point of minimum approach with respect to the beam axis) and the extrapolated intercept of the fitted track with the outer layer of the COT in case of success.

The XFT performance has been defined to match and generally improve on the performances of the corresponding device used during Run I (the Central Fast Tracker or CFT). To this end, the main design goals are ${ }^{3}$ :

\footnotetext{
${ }^{1}$ Although calorimeter responses are digitized into 10-bit words, allowing a precision of $125 \mathrm{MeV}$, at Level 1 only a precision of $250 \mathrm{MeV}$ is available.

${ }^{2}$ Except for the highest $|\eta|$ towers, whose coverage is $\Delta \eta \simeq 1$.

${ }^{3}$ The corresponding measured performances for CFT are reported in brackets for comparison.
} 

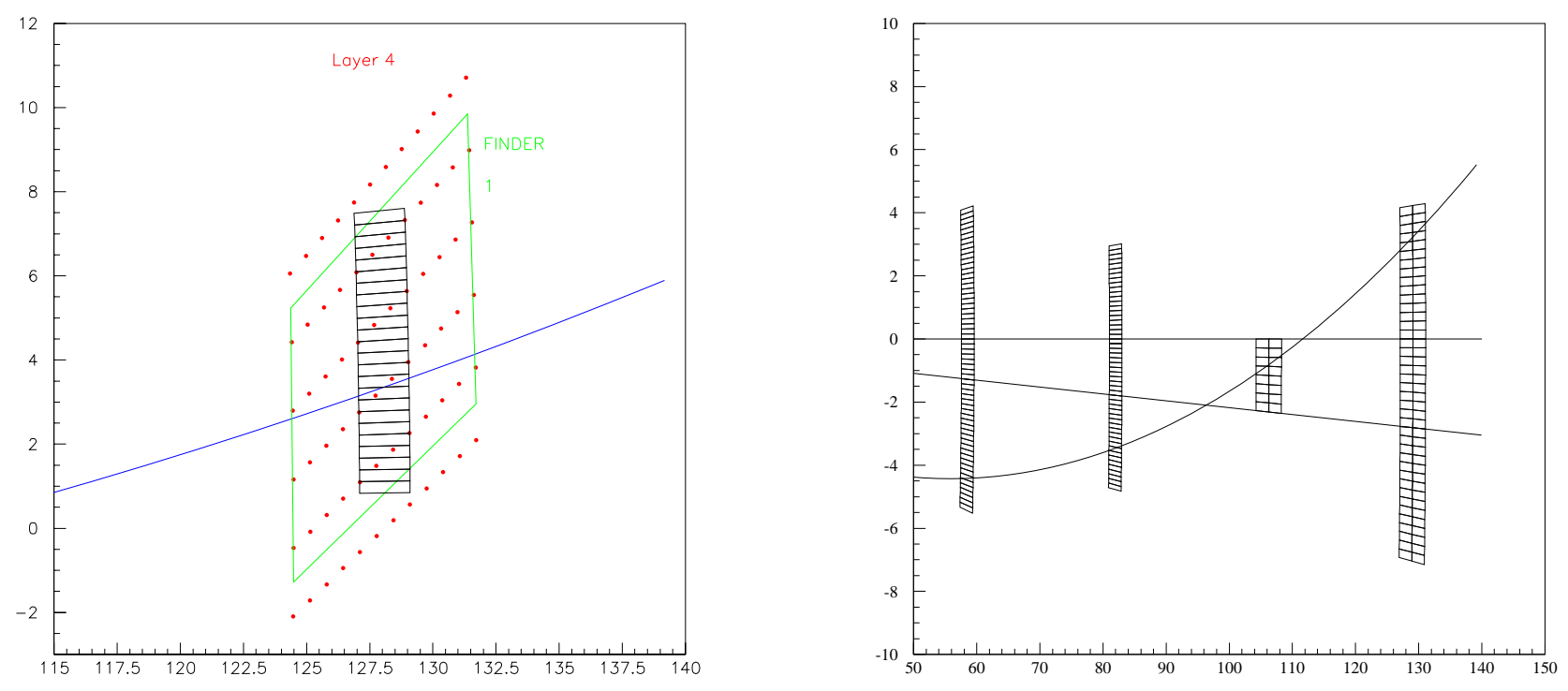

Figure 3.3: Graphical representation of XFT track segment finder (left) and XFT track segment linker (right).

$\diamond$ track-finding efficiency better than $96 \%$ for tracks with $p_{T}>1.5 \mathrm{GeV} / \mathrm{c}(\sim 70 \%$ for tracks with $p_{T} \gtrsim 2.2 \mathrm{GeV} / \mathrm{c}, \sim 100 \%$ for $p_{T} \gtrsim 3.5 \mathrm{GeV} / \mathrm{c}$ );

$\diamond \delta p_{T} / p_{T}^{2}<2 \%(\mathrm{GeV} / \mathrm{c})^{-1}\left(\delta p_{T} / p_{T}^{2}<3.5 \%(\mathrm{GeV} / \mathrm{c})^{-1}\right)$

$\diamond$ double fake-rate rejection with respect to the CFT.

A good resolution on the fitted track $\phi_{0}\left(\delta \phi_{0} \lesssim 8 \mathrm{mrad}\right)$ is required if the XFT output is to be used as a seed for the Silicon Vertex Tracker, a Level 2 trigger algorithm that will be described later in this chapter.

The XFT works on the basis of the following procedure:

1. track segments are identified in each axial superlayer of the cot ${ }^{4}$. A track segment is searched for by a pattern recognition algorithm among all possible hit patterns achievable by a track with $p_{T} \geqslant 1.5 \mathrm{GeV} / \mathrm{c}$ in the 12 layers of sense wires of each of four adjacent COT cells (see Fig. 3.3 left side).

2. track segments are linked together into tracks. Track segments (pixels) are defined by their $\phi$ position at superlayer 3 and by their slope ${ }^{5}$. The starting point of the linking procedure is a valid pixel in superlayer 3 , corresponding to a $1.25^{\circ}$-wide COT slice (see Fig. 3.3 right side); starting from this pixel, all combinations of track segments $\left(\right.$ roads) compatible with a $p_{T} \geqslant 1.5 \mathrm{GeV} / \mathrm{c}$-track hypothesis are computed through different superlayers. Among all roads found in each $1.25^{\circ}$ COT slice, the

\footnotetext{
${ }^{4}$ Here numbered between 1 (inner) and 4 (outer)

${ }^{5}$ Slope is determined by the two outer superlayers only.
} 
one with the greatest number of associated pixels ${ }^{6}$ and highest $p_{T}$ is returned by the algorithm together with its charge, $p_{T}$ and $\phi$ at superlayer 3.

Once the tracks have been found, their information is sent to the extrapolation unit (XTRP), whose task primarily consists in mapping the XFT tracks by means of lookup tables onto muon and electron primitives found by other Level 1 trigger processors; in other words, the XTRP provides an extrapolation of each XFT track to the central calorimeter wedges $\left(15^{\circ}\right.$ wide) and to the muon system (CMU and CMX). At the same time, for each track, the $\phi, p_{T}$ information is made available to Level 2 processors, such as SVT.

Furthermore, the XTRP can generate itself a Level 1 trigger accept, according to number of tracks, their topology and $p_{T}$ threshold.

\section{$\underline{\text { Muons }}$}

The Level 1 muon trigger is aimed at providing single and dimuon objects for the Level 1 trigger decision; a Level 1 trigger muon object is obtained by matching a tracking primitive (a XFT track) to a muon primitive.

The definition of muon primitive depends on the specific muon detector type; for scintillators (CSP, CSX), a muon primitive corresponds either to single-hits or to coincidences of hits. For wire chambers (CMU, CMP, CMX), a cluster of hits $(s t u b)$ is searched for; in the case of $\mathrm{CMU}$ and $\mathrm{CMX}$, a stub is defined whenever a coincidence, within a given time ${ }^{7}$, is achieved between two or more hits collected in projective wires belonging to different radial layers. For CMP, on the other hand, only a pattern of hits in a tube stack consistent with a traversing track is required for stub definition. A further muon primitive is supplied by the hadron calorimeter, which is capable of signalling the passing of a minimum ionizing particle in each trigger tower.

Two major improvements distinguish the Run II from the Run I muon trigger:

$\diamond$ an increased coverage for CMP $(+17 \%)$ and CMX $(+50 \%)$, which results both in an enhanced purity of the sample collected by combining CMU and CMP primitives and in a larger global acceptance;

$\diamond$ central tracking information from the XFT-XTRP is already available at Level 1, providing a more effective fake-rate rejection;

As previously mentioned, XFT tracks and muon stubs information can be merged into muon objects by means of a matching between trigger primitives; these procedures consists of a $r-\phi$ track-stub match ${ }^{8}$ and of a $p_{T}$-based match, since independent thresholds can be set on the $p_{T}$ of the track and of the $\mathrm{stub}^{9}$. In order to avoid a double-counting when considering dimuon objects, at least an empty $\Delta \phi=2.5^{\circ}$ segment is required between stubs.

\footnotetext{
${ }^{6}$ The possibility of a three-out-of-four match is permitted for 'short tracks', which do not reach the outer superlayer; large $|\eta|$ tracks are included into this definition.

${ }^{7} p_{T}$ requirements for stubs are achieved by adjusting the time window in the stub definition.

${ }^{8}$ At Level 1 the azimuthal matching resolution is $2.5^{\circ}$, only half of the full detector azimuthal granularity.

${ }^{9}$ However, it should be kept in mind that the precision on the $p_{T}$ measurement achieved by the differential timing in the muon chambers is lower than the corresponding quantity measured by the XTRP.
} 


\section{$\underline{\text { Electrons }}$}

As in the case of muons, electron triggers depend on XFT primitives; in this case, however, the additional electron-oriented primitives available at Level 1 are essentially the calorimeter towers. A Level 1 electron trigger can be obtained by requiring a trigger tower with an electromagnetic energy content above a certain threshold to be matched to a XFT track fulfilling some $p_{T}$ requirement. Rate constraints effectively influence the possibilities one can pursue in choosing thresholds: in particular, one can hope to lower the energy/momentum thresholds only by considering a two-electron topology in order to recover an acceptable trigger rate.

\subsubsection{Level 2}

Level 2 selection procedures are more sophisticated than those used at Level 1. While Level 1 is primarily devoted to rate reduction, the less stringent time constraints $(\sim 20 \mu \mathrm{s})$ of Level 2, together with considerably lower rate, allows the emphasis to be put on the improvement of the signal to background ratio.

Though the core of the tools available at Level 2 consists of hardwired procedures, they are incremented with a set of time-optimized software.

\section{Calorimetry}

One of the most common features of high energy hadron collisions final states is jets: they originate from the hadronization of energetic partons, when, as a consequence of the Lorentz boost, the particles produced in such a process tend to be compressed into a narrow region of phase-space.

Since in general jets are not expected to be fully contained in a single trigger tower, the energy thresholds for Level 1 jet trigger requirements have to be set at a considerably lower values than the typical jet energy in order not to loose efficiency. This, however, implies trigger rates which are too high to be fed directly into Level 3.

Effective rate reduction can be achieved at Level 2 by considering the energy associated with clusters of contiguous trigger towers instead of single trigger tower energies ${ }^{10}$. The definition of clusters within an event requires an algorithm (cluster finder) whose working principle $^{11}$ can be explained in terms of four steps (a graphical representation of which can be found in fig. 3.4).

Step 1 The cluster finder needs two energy thresholds to be set: the seed threshold defines which trigger towers are used as a starting point by the algorithm; the shoulder threshold establish the duration of a recursive procedure which will be described in the third step. The set of a particular seed and shoulder thresholds is addressed as a "pass" which identifies clusters with similar topologies.

Step 2 All trigger towers with energy content above the seed threshold are identified and recorded as 'seed towers'; when all seed towers have been found, a second loop is performed on the remaining trigger towers in order to tag the 'shoulder towers', that is, the trigger towers whose energy exceeds the shoulder threshold.

\footnotetext{
${ }^{10}$ Here and in the following, the term 'energy' indicates the $E_{T}$.

${ }^{11}$ Essentially unchanged from Run I.
} 
Step 3 An iterative procedure begins with the seed tower that has the lowest $\phi$ address $\left(\phi_{s}\right)$ among all seed towers with lowest $\eta$ address $\left(\eta_{s}\right)$. The four $\eta=\eta_{s} \pm 1$ and $\phi=\phi_{s} \pm 1$ neighbouring trigger towers are then examined. If one of these trigger towers is a shoulder tower ${ }^{12}$, then, after being flagged as 'found', its three neighbouring trigger towers are also examined. The procedure is repeated until no more contiguous shoulder towers are found.

Step 4 Step 3 is repeated for all seed towers not flagged as 'found' in previous iterations.

When the procedure comes to an end, clusters are identified with the groups of contiguous trigger tower that have developed around a seed tower; each cluster is assigned an energy equal to the sum of the energies ${ }^{13}$ of all trigger towers belonging to the same group. The $\eta-\phi$ position of each cluster is then identified with the $\eta-\phi$ position of the seed tower which initiated the iterative procedure.

Within each event, the cluster finder procedure will be performed for each pass that has been defined; the four passes that have been defined for Run II are:

$\diamond$ type 1: $E_{T}^{e m}($ seed $)=3.0 \mathrm{GeV}, E_{T}^{e m}($ shoulder $)=1.0 \mathrm{GeV}$ and $E_{T}^{\text {had }}($ seed,shoulder $)=\infty$ for low- $p_{T}$ electron/photon;

$\diamond$ type $2: E_{T}^{e m}($ seed $)=8.0 \mathrm{GeV}, E_{T}^{e m}($ shoulder $)=7.5 \mathrm{GeV}$ and $E_{T}^{\text {had }}($ seed,shoulder $)=\infty$ for high- $p_{T}$ electron/photon;

$\diamond$ type 3: $E_{T}($ seed $)=3.0 \mathrm{GeV}, E_{T}($ shoulder $)=1.0 \mathrm{GeV}$ (both hadronic and electromagnetic components) for jet clustering;

$\diamond$ type 4: $E_{T}^{\text {em }}($ seed $)=2.0 \mathrm{GeV}, E_{T}^{e m}($ shoulder $)=\infty$ and $E_{T}^{\text {had }}($ seed,shoulder $)=\infty$ for low- $p_{T}$ electron in B-physics.

In the case of type 4 clusters, the choice of an infinite electromagnetic shoulder threshold essentially corresponds to an implicit isolation requirement, since this procedure will lead to single trigger tower clusters. Isolation patterns can be explicitly required during clustering by asking the smallest of the sums depicted in fig. 3.5 to be less than a given threshold; however, for low- $p_{T}$ electrons a type 4 clustering is preferred, since, assuming the identified cluster correctly tags the tower struck by the electron, any further tower contribution to the energy would dilute the information deposited in the calorimeter by the electron.

Exactly as for Level 1, Level 2 calorimetry primitives can be grouped into two categories:

- object primitives. Energy depositions ${ }^{14}$ associated with hadronic (type 3) or electromagnetic (type 1, 2 and 4) clusters. Besides energy content and type, the information concerning clusters' position $(\eta-\phi$ address of seed towers) is stored, in order to enable combinations between calorimetric and tracking (XFT) primitives;

\footnotetext{
${ }^{12}$ Note that seed towers also fulfill the shoulder condition, unless the shoulder threshold is higher than the seed threshold.

${ }^{13}$ Electromagnetic and total contributions separately.

${ }^{14}$ At Level 2, the full calorimeter resolution of $125 \mathrm{MeV}$ is exploited.
} 


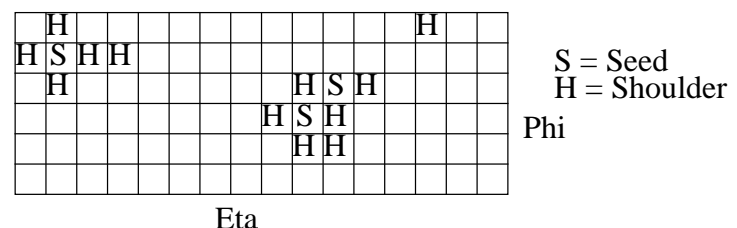

Step 1: seed and shoulder trigger tower tagging

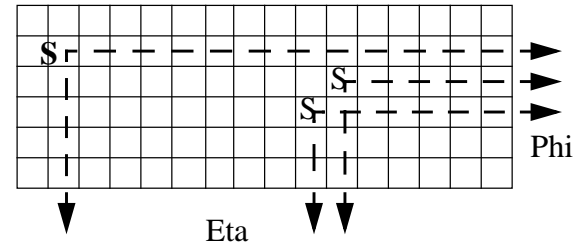

Step 2: seed and shoulder trigger tower eta-phi address recording

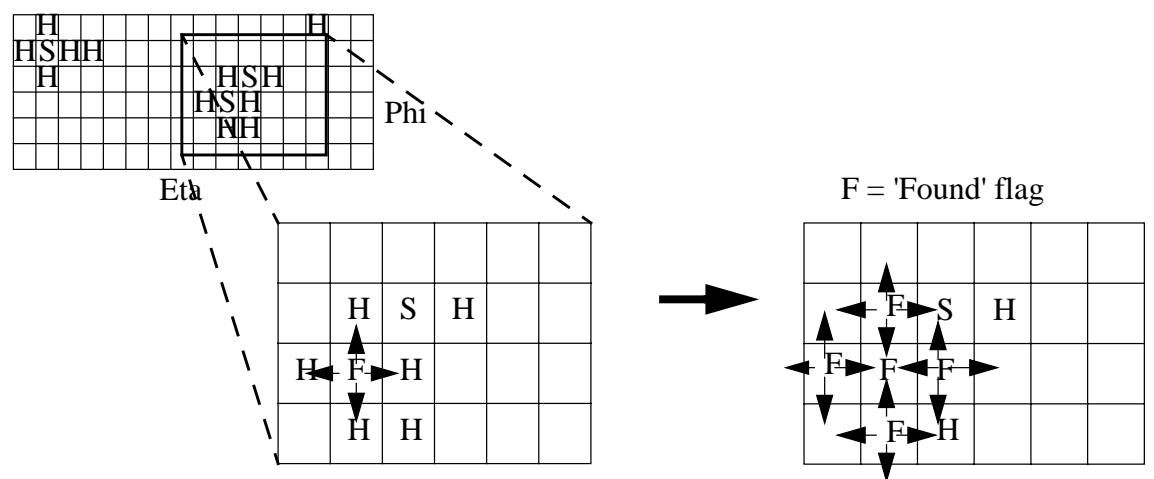

Step 3: contiguous shoulder towers are merged into clusters

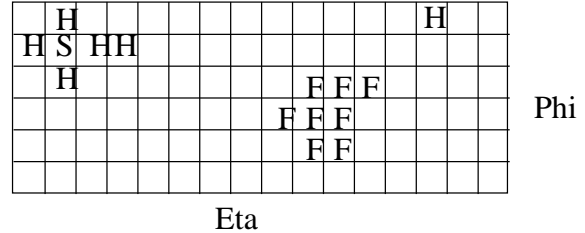

Figure 3.4: Cluster finding procedure.

S: seed tower $(\mathrm{EM}+\mathrm{HAD}) \quad \mathrm{X}$ : summed towers
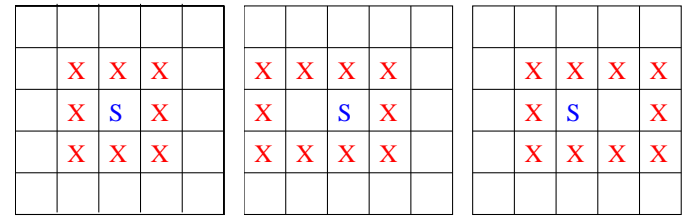

\begin{tabular}{|l|l|l|l|l|}
\hline & $X$ & $X$ & $X$ & \\
\hline & $X$ & & $X$ & \\
\hline & $X$ & $S$ & $X$ & \\
\hline & $X$ & $X$ & $X$ & \\
\hline & & & & \\
\hline
\end{tabular}

\begin{tabular}{l|l|l|l|l|}
\hline & & & & \\
\hline & $X$ & $X$ & $X$ & \\
\hline & $X$ & $S$ & $X$ & \\
\hline & $X$ & & $X$ & \\
\hline & $X$ & $X$ & $X$ & \\
\hline
\end{tabular}

Figure 3.5: Tower isolation patterns. 
- global primitives. The number of clusters, according to cluster type and energy, is available at Level 2. Global energy sums are now cluster-based, i.e. they are obtained by summing the contributions of all cluster in an event; the total $\Sigma E_{T}$ is performed over all type 3 clusters, while other $\Sigma E_{T}$ observables can be defined considering different clusters subsets (for instance, on can compute the sum over all type 3 clusters with $E_{T}$ greater than a given minimum threshold). Global non clusterized energies are available at Level 1 only.

\section{$\underline{\text { SVT }}$}

Already during Run I, CDF, being equipped with a vertex detector, was capable of performing B-physics studies, which essentially rely on reconstructing secondary vertices which are produced as a consequence of the large B-hadron decay length $(\sim 500 \mu \mathrm{m})$. However, during Run I this capability was confined to the offline analysis, while the on-line tagging of the interesting events was restricted to the use of leptonic triggers; for this reason, the global efficiency on B-physics was drastically reduced, while some specific processes (for instance, $\mathrm{B}^{0} \rightarrow \pi^{+} \pi^{-}$) important for $C P$ violations measurements - were virtually undetectable.

During Run II, this deficiency was remedied by the introduction of the SVT (Silicon Vertex Tracker [2][3]), a device for tagging displaced tracks already at trigger level; in fact, displaced tracks (with large impact parameter) can be interpreted as signals of the existence of secondary decay vertices of heavy-flavour objects. This tool provides a response, by merging the information supplied by the new silicon vertex detector (SVXII) with the output of the Level 1 fast tracker (XFT), in time for the Level 2 decision; in this way, the SVT allows for enhanced collection of fully hadronic decay modes of B-hadrons as well as an efficiency enhancement for the semileptonic channels by enabling a lower $p_{T}$ threshold for lepton tagging.

The working strategy of SVT is summarized in fig. 3.6. First, SvXII channels, grouped into $24 \phi$ sectors (each one of the 12 azimuthal wedges is divided in two sectors according to the sign of $z$ ), are read out by 72 Hit Finders, which perform pedestal and bad-channel subtraction; once strip readout is completed, the Hit Finders search for hit clusters in each layer contained in the corresponding sectors, computing the centroid of each admissible cluster. The centroids represent the most likely intersection points of the trajectory of a track with each of the five ${ }^{15}$ radial silicon layers of svXII. Silicon cluster information is then transmitted to the Associative Memory Sequencer (AMS), which, at the same time, is fed with the XTRP output; in the AMS, a first, tentative association between clusters and XFT tracks takes place: this is done by lining up the clusters of a given $\phi$ sector with outer XFT tracks. Due to the large number of possible combinations arising from this procedure, each cluster is substituted by a superstrip, whose dimension $(250 \mu \mathrm{m})$ represents the best compromise between fake track rejection and cost. Then, the association between stacks of superstrips and XFT tracks is performed: each admissible combination, evaluated on the basis of lookup tables, defines a road, which represents a broad track. Roads, each corresponding to a set of four SVXII clusters and an outer XFT track, are then sent to the Hit Buffers, which retrieve the full detector information (i.e. the single hits coordinates for each SVXII cluster and the two XFT track parameters ${ }^{16}$ ) to be used to fit the track to

\footnotetext{
${ }^{15}$ Only four are used by SVT.

${ }^{16}$ Namely, the signed curvature and the $\phi$ seed (i.e. the azimuthal coordinate of the track at axial
} 
an arc of circumference of the trajectory and obtain the three parameters $p_{T}, \phi$ and $d$ (impact parameter).

\section{$\underline{\text { Muons }}$}

Minor differences distinguish Level 2 from Level 1 muon primitives; this difference is essentially related to the precision of the $\phi$-matching between XFT tracks and stubs. At Level 2, in fact, full detector resolution is exploited and the matching is performed within $1.25^{\circ}$ for $\mathrm{CMU}$ and $\mathrm{CMX}$, while for CMP the track segment to be matched to the XFT track has to be reconstructed within one-tube stack, which corresponds to an azimuthal coverage of $1.25^{\circ}$ at the closest radial distance from the beam axis, reducing to $0.6^{\circ}$ towards the edges of the chamber.

\section{Electrons}

At Level 2, central and plug shower maximum (XCES and XPES) primitives are available for triggering on electrons and photons; the shower maximum detectors rely on the strip/wire chambers (CES) contained in the central electromagnetic calorimeter towers and on twolayers of scintillator strips (PES) located within the electromagnetic plug calorimeters.

Both detectors provide a measurement of the charge deposition as well as a determination of the position of the intersection point of the track trajectory at the detector surface; this is achieved by merging the information collected by strip pads and wires in the central region or by $\mathrm{U}$ and $\mathrm{V}$ plug scintillators strips (see fig. 2.14).

The purpose of these detectors is two-fold: first, providing rate-rejection handle against non-electromagnetic matter and, second, enabling a separation between electrons and photons.

XCES Although the readout electronics has been completely renewed, both detector specifications and XCES trigger working scheme remain substantially the same for Run II as for Run I.

Only the signals collected by the wires are used at trigger level; the procedure for computing the XCES primitive follows from the shower maximum detector geometry: each calorimeter wedge $\left(\Delta \phi=15^{\circ}, \eta \gtrless 0\right)$ contains two strip chamber modules, each subtending a $\Delta \eta \sim 0.55$ region. There are 32 wires, all parallel to the $z$ axis and spanning the whole width of a sector, belonging to each module; these are divided into eight groups (bits) of four adjacent wires. The pulse-heights of the wires within the same bit are summed together and compared to a threshold; if at least one of the two bits (one per CES module) corresponding to the same average $\phi$ value fulfills the minimum pulse-height requirement, the corresponding XCES bit is set. Each bit is compared to two thresholds: high $p_{T}$ threshold for electrons and low one for photons. In this way a total of 768 xCES bits are set $(2-$ modules $\times 24$ - wedges $\times 8-$ bits $\times 2$ - thresholds $)$.

For triggering electrons the XCES primitive is usually matched with an XFT track in $\phi$. $\phi$-matching can achieve a $2^{\circ}$ resolution, which is $\sim 8$ times finer than a track-tower matching. This results into a $\sim 50 \%$ background reduction, while $\sim 90 \%$ efficiency

superlayer 3 of the COT). 


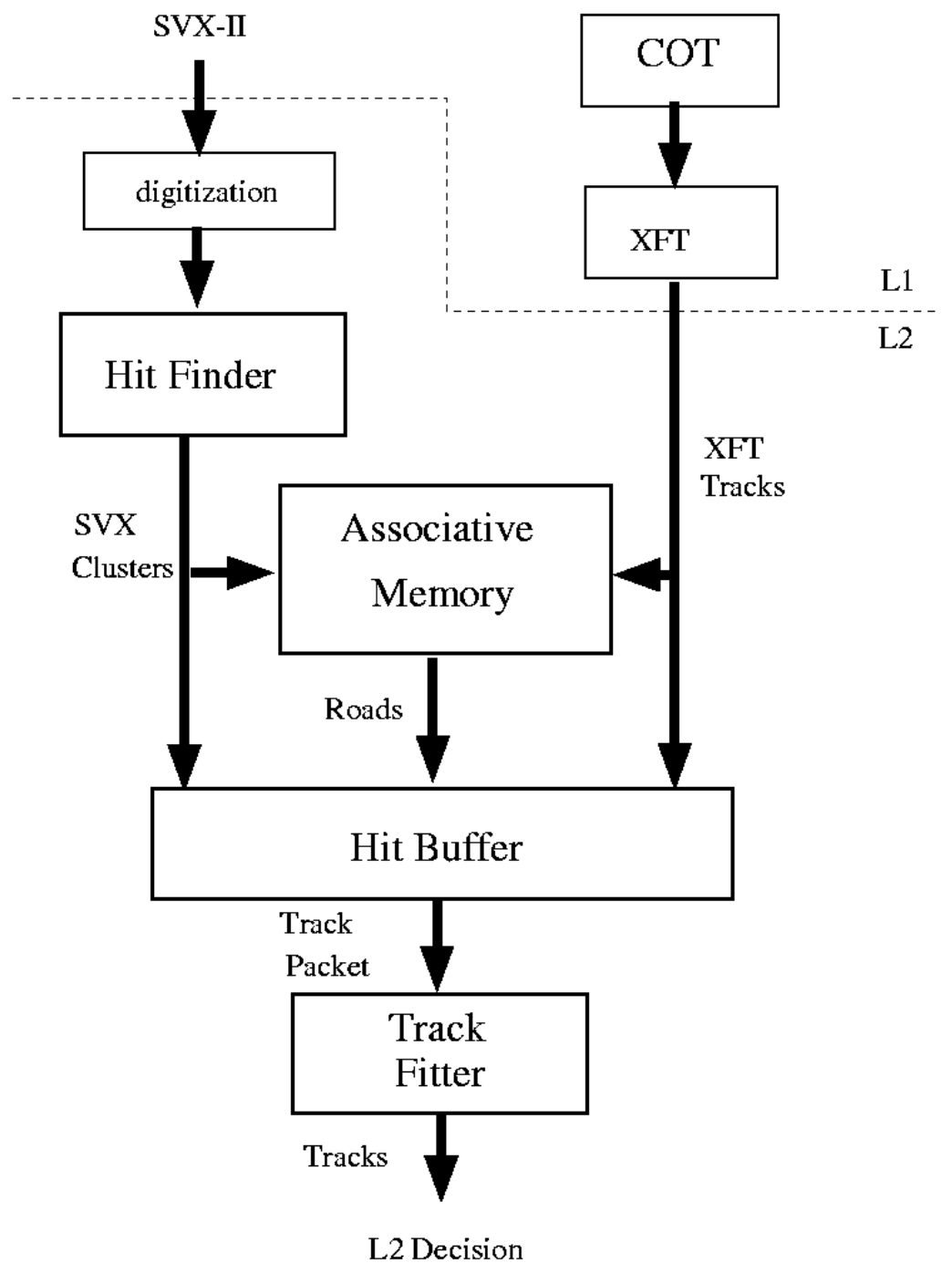

Figure 3.6: The SVT data flow. 
on signal is retained. Triggering upon photons involves a requirement on the pulseheight, while XFT tracking can be used as a veto for charged tracks extrapolating in the vicinity of the XCES bit.

XPES The PES is a new detector, with a different geometry and a different way of functioning than the CES. Besides, most of its acceptance, covering $1.13<|\eta|<3.50$, falls outside the tracking region (which extends up to $|\eta| \simeq 2$ ).

The PES has not yet been used for Level 2 triggering, but the possible options for exploiting its information at this stage of the selection are mainly based on the following idea: signals collected by groups of scintillator strips are compared to threshold values and, eventually, correlated to signals collected by the corresponding plug calorimeter towers.

\subsubsection{Level 3}

Physics objects (i.e. variables closely related to the kinematics of physics processes) are of fundamental importance for selecting a signal from the bulk of background events produced in hadronic collisions; however, at trigger level, especially at Level 1 and 2, all available information is not fully exploited due to time constraints. The third level of the trigger provides a further step towards a refined selection, augmenting the event purity and lowering the rate to a level compatible with storage procedures.

At Level 3, a more sophisticated event reconstruction is performed; this implies the definition of a new class of high level physics objects, including three-dimensional tracks, jets and identified leptons. Some variables which need a long processing time (like, for instance, global kinematical event observables, or track isolation properties), cannot be calculated within the allowed time slot at trigger level. Further offline processing is then performed on the selected events. Nevertheless, in order to ensure a good agreement between the response of the trigger and the selection of the offline analyses, the Level 3 code, which is completely software based, is grounded on the offline production reconstruction code.

The physics objects of the offline reconstruction will be described in the next chapter.

\subsection{The SUSY DILEPTON triggers}

The physics motivation for a data set characterized by the presence of identified dileptons comes from the fact that many unexcluded parts of the SUSY paramenter space include processes producing leptons of relatively low $p_{T}(<20 \mathrm{GeV} / \mathrm{c})$. The dilepton (electrons and muons) triggers dedicated to exotic searches were originally proposed in [1] and have been active since the beginning of CDF Run II (trigger structure in table 3.2). In Summer 2002 a revision of these triggers were proposed in [4] mainly aimed at simplifying the procedure for estimating the trigger efficiencies and in August 2003 the new architecture of the triggers were implemented in the CDF trigger system (updated trigger structure in table 3.3). The work presented in this thesis ${ }^{17}$ represents the beginning of an analysis originally motivated by this strategy so that a more detailed description of this approach is given in the next section.

\footnotetext{
${ }^{17}$ Because of time constraints this thesis focuses on one trigger path. Other paths will be analyzed subsequently.
} 
During 2004 the increasing luminosity led to higher rates and effective cross sections ${ }^{18}$ of many of the trigger paths of the CDF trigger system. Of particular concern was the Level 2 bandwidth. During this period a big effort was made by the collaboration [5] to find additional trigger requirements which would reduce the corresponding effective trigger cross sections. In a common effort to increase rejection while keeping high signal efficiency, parallel studies were undertaken to re-think and simplify the SUSY DILEPTON TRIGGER structure [6]. A guiding principle was to conform the trigger requirements at all trigger levels: a single lepton requirement at Level 1 is now followed by a single lepton requirement at Level 2 and 3 also. In order to reach tolerable trigger bandwiths at high luminosity, this approach also needed to adapt the lepton $p_{T}$ thresholds.

\begin{tabular}{l}
\hline \hline CEM4_CMUP4 \\
CEM4_CMX4 \\
CEM8_PEM8 \\
CMUP4_PEM8 \\
CMX4_PEM8 \\
DIELECTRON_CENTRAL_4 \\
DIMUON_CMUP4_CMX4 \\
DIMUON_CMUPCMUP4 \\
\hline \hline
\end{tabular}

Table 3.2: List of SUSY DILEPTON triggers before August 2003. The name of each trigger path corresponds to the "types" of leptons required.

\subsubsection{The SUSY DILEPTON trigger strategy}

The revision proposed in [4] for the SUSY DILEPTON trigger is aimed at measuring trigger efficiencies for each single lepton type ${ }^{19}$ involved in the trigger and combining then these single lepton efficiencies in order to obtain the efficiencies of the trigger paths. This approach might have become complicated because the definition of a lepton at Level 1 and 2 generally depended on the trigger path considered. This problem was overcome by standardizing the definition of each lepton type in order to minimize the number of single lepton objects one has to deal with. Since all the trigger paths incorporate the same Level 3 single lepton definitions, the standardization of lepton definitions is needed only at Level 1 and Level 2. This approach led to 6 lepton types: three electrons and three muons (see Tab. 3.4).

By making the trigger selection at Level 3 completely efficient w.r.t. events that satisfy the offline standard selection, the efficiency of each lepton can then be written as $\epsilon_{T R G} \times \epsilon_{O F F}$, where the first factor is the efficiency of the single lepton at Level 1 and 2 and the second factor is the efficiency of the offline lepton selection.

The problem with evaluating single lepton efficiencies from a sample of dilepton

\footnotetext{
${ }^{18}$ The effective cross section of a trigger path increases because of rate-dependent accidentals.

${ }^{19}$ The lepton "type" (e.q.CEM4) refers to Level 3 and indicates the subdetector (CEM stands for central EM calorimeter) where the lepton is detected and the $p_{T}$ threshold (CEM4 implies a $p_{T}$ threshold of $4 \mathrm{GeV} / \mathrm{c}$ ) at Level 3 . However it is also associated with the requirements defined at Level 1 and Level 2 .
} 


\begin{tabular}{|c|c|}
\hline 2 low $p_{T}$ leptons & $\begin{array}{l}\text { CEM4_CMU4 } \\
\text { CEM4_CMUP4 } \\
\text { CEM4_CMX4 } \\
\text { CEM4_PEM8 } \\
\text { CEM8_PEM8 } \star \\
\text { CMU4_PEM8 } \\
\text { CMUP4_PEM8 } \star \\
\text { CMX4_PEM8 } \\
\text { DIELECTRON_CENTRAL_4 } \\
\text { DIMUON_CMU4_CMX4 } \\
\text { DIMUON_CMUCMU4 } \\
\text { DIMUON_CMUP4_CMX4 } \\
\text { DIMUON_CMUPCMUP4 }\end{array}$ \\
\hline medium $p_{T}$ lepton + track & $\begin{array}{l}\text { CEM4_CMU4_L2_CEM8_PT8_CES2_\&_TRK8 } \\
\text { CEM4_CMX4_L2_CEM8_PT8_CES2_\&_TRK8 } \\
\text { DIELECTRON_CENTRAL_4_L2_CEM8_PT8_CES2_\&_TRK8 }\end{array}$ \\
\hline inclusive high $p_{T}$ lepton & $\begin{array}{l}\text { CEM4_CMU4_L2_TRK8_L1_CMUP6_PT4 } \\
\text { CMU4_PEM8_L2_TRK8_L1_CMUP6_PT4 } \\
\text { DIMUON_CMUCMU4_L2_TRK8_L1_CMUP6_PT4 } \\
\text { DIMUON_CMU4_CMX4_L2_TRK8_L1_CMUP6_PT4 } \\
\text { CEM4_PEM8_L2_CEM12_PT8 } \\
\text { DIELECTRON_CENTRAL_4_L2_CEM12_PT8 }\end{array}$ \\
\hline
\end{tabular}

Table 3.3: SUSY DILEPTON triggers after August 2003. Since April 2004 triggers marked with $\star$ were removed because of rate restrictions, the CES threshold was raised from 2 to 3 GeV and the L2 muon code was implemented. The last change before the 2004 shutdown took place in June 2004 when L2_CEM12_PT8 was replaced with L2_CEM8_PT8_DPS.

events $^{20}$ can then be overcome by adding suitable single lepton Level 1 and Level 2 backup paths (see "inclusive high $p_{T}$ lepton" trigger in Tab. 3.3). It should be noted that keeping the analysis and calibration data set in the same sample provides a simple way to account for the detector conditions during data-taking which are then naturally folded into the efficiency calculations.

Another category of trigger paths has been added in order to enhance the acceptance by recovering possible inefficiencies coming from erroneous associations of tracks with the energy depositions in the calorimeter or to the hits in the muon chambers or from leptons leaking through detector gaps. This last category requires a single lepton at Level 1 and Level 2 with the presence of an additional track (see "medium $p_{T}$ lepton + track" trigger in Tab. 3.3). A tighter $p_{T}$ requirement is needed for both the single-lepton and the lepton+track paths in order to accomodate the available bandwidths.

With this layout, as far as the Level 1 and Level 2 are concerned the SUSY DILEPTON triggers can be subdivided in three categories (see also the structure of table 3.3):

- 2 low $p_{T}$ leptons $(4 \mathrm{GeV} / \mathrm{c})$

\footnotetext{
${ }^{20}$ i.e. the bias introduced by the second lepton requirement because all paths terminate in a di-lepton requirement at Level 3.
} 
- medium $p_{T}$ lepton $+\operatorname{track}(8 \mathrm{GeV} / \mathrm{c})$

- inclusive high $p_{T}$ lepton $(8 / 12 \mathrm{GeV} / \mathrm{c})$

\begin{tabular}{l|l|l}
\hline \hline Lepton type & L1 and L2 Trigger & Lepton type definition \\
\hline \hline CEM4 & L1_CEM4_PT4 \&\& & central electron with $p_{T}>4 \mathrm{GeV} / \mathrm{c}$ \\
& L2_CEM4_PT4_CES2(3) & \\
\hline CEM8 & L1_CEM8_PT8 \&\& & central electron with $p_{T}>8 \mathrm{GeV} / \mathrm{c}$ \\
& L1_CEM8_PT8_CES2 $(3)$ & \\
\hline PEM8 & L1_CMU \&\& L2_PEM8 & forward electron with $p_{T}>8 \mathrm{GeV} / \mathrm{c}$ \\
\hline CMUP4 & L1_CMUP6_PT4 & central muon with $p_{T}>4 \mathrm{GeV} / \mathrm{c}$ (at Level 3) \\
\hline CMX4 & L1_CMX1.5_PT2_CSX & $\begin{array}{l}\text { central muon with } p_{T}>4 \mathrm{GeV} / \mathrm{c} \\
\text { also using the outer } \mathrm{CMP} \text { muon chamber. }\end{array}$ \\
\hline \hline
\end{tabular}

Table 3.4: Standard lepton "type" definitions. The trigger primitives indicated in the middle column correspond to the trigger requirements at Level 1 and 2 for each lepton type. 


\section{Bibliography}

[1] M. Albrow et Al., (The CDF Trigger and Datasets Working Group), Run II Trigger Table and Datasets Plan, CDF internal note 4718, Sep 2000.

[2] S. Belforte et Al., SVT - Silicon Vertex Tracker - Technical Design Report, CDF internal note 3108, Oct 1996.

[3] SVT upgrade group, Silicon Vertex Trigger Upgrade, CDF internal note 7064, 2004.

[4] M.P.Giordani, S.Lammel, Dilepton Triggers for Exotic Searches at CDF II, CDF internal note 6074, Aug 2002.

[5] T. Akimoto at Al., Exotics Trigger Proposal for 2005, CDF internal note 7441, 2005.

[6] M. Rossi et Al., Study of the trigger rates for updated SUSY dilepton trigger paths, CDF internal note 7413, 2005. 



\section{Chapter 4}

\section{Event reconstruction}

Offline physics analyses are based on the reconstruction of physics objects from raw data produced in hadron collisions. Once events are selected by means of the trigger the raw information coming from the several components of the detector must be processed in order to identify particles produced in the hadron interaction.

In this chapter a brief review of the main physics objects (tracks and jets) used for event reconstruction at $\mathrm{CDF}$ is presented. Moreover a description of how to exploit them in order to identify leptons is given.

\subsection{Tracks}

Tracks carrying an electric charge $q e$ (where $e$ is the positron charge) travelling with a velocity $\boldsymbol{v}$ in a homogeneous magnetic field $\boldsymbol{B}$ experience a Lorentz force:

$$
\boldsymbol{F}=q e \boldsymbol{v} \wedge \boldsymbol{B}
$$

that constrains the tracks to a helicoidal trajectory, whose radius $\rho$, measured in the plane transverse to $\boldsymbol{B}$, is directly related to the track transverse momentum $p_{T}$ according to the relation:

$$
\rho=\frac{p_{T}}{|q| e B} .
$$

A track trajectory is completely defined by five parameters:

$\cot \theta$ : cotangent of the polar angle of the helix measured at minimum approach to beam axis;

$C$ : signed half curvature (same sign of $q$ );

$z_{0}: z$-coordinate of minimum approach to beam axis;

$d$ : impact parameter (minimum distance of the helix from the beam axis in the $(x, y)$ plane with sign defined according to Fig. 4.1); if $\left(x_{0}, y_{0}\right)$ is the origin of the projected circle of helix in the $(x, y)$ plane, then the impact parameter is calculated as (see Fig. 4.2)

$$
d=Q\left(\sqrt{x_{0}^{2}+y_{0}^{2}}-\rho\right)
$$

where $\rho=\frac{1}{|2 C|}=\frac{1}{2 Q C}$ is the radius of the circle and $\mathrm{Q}$ the sign of the particle charge; 
$\varphi_{0}$ : azimuthal angle of helix at minimum approach to beam axis.

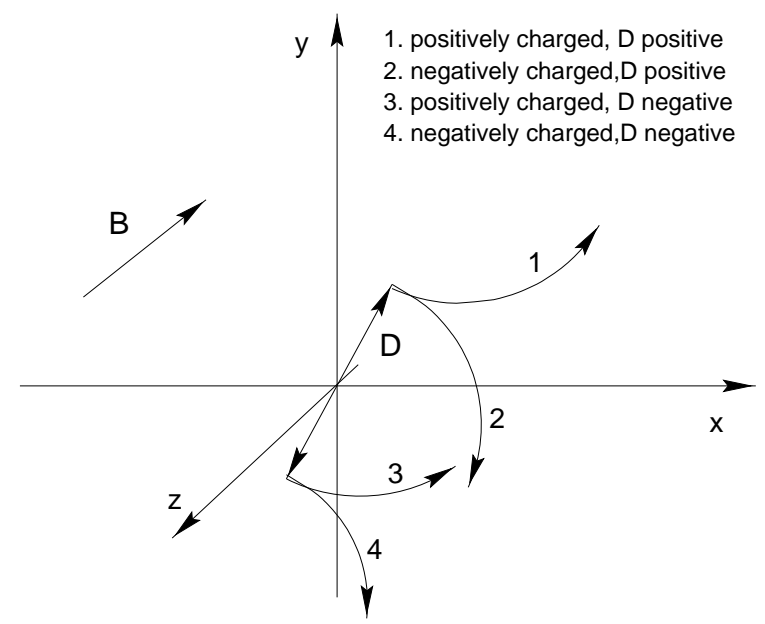

Figure 4.1: Sign convention for impact parameter.

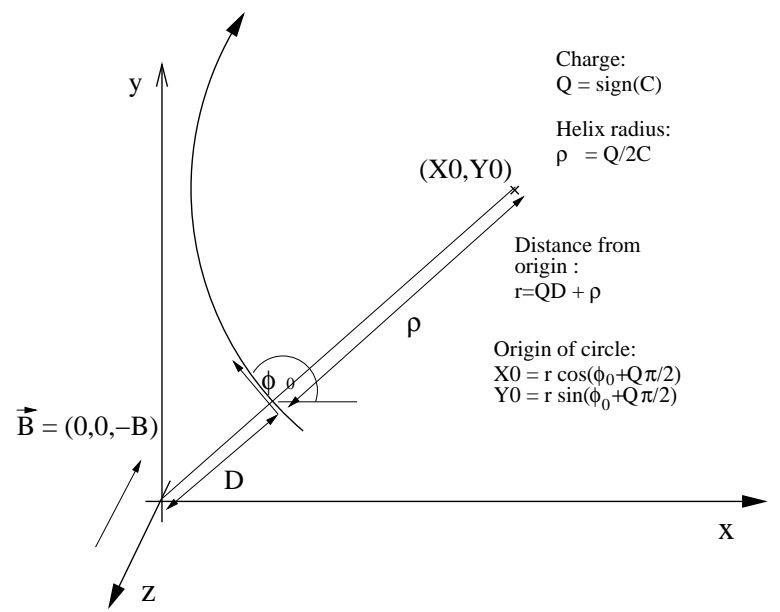

Figure 4.2: Representation of the track parameters used at CDF.

\subsubsection{Reconstruction}

The CDF tracking system for Run II has been upgraded in order to fit a higher luminosity scenario and, at the same time, to correct for the limitations observed during Run I. For this purpose, the CTC and the SVX have been substituted by the COT and a new silicon inner tracker respectively.

The adoption of the COT, a tracking chamber which addresses the increased luminosity and reduced bunch spacing by adopting smaller drift cells, provides a faster response and double stereo sampling with respect to Run I configuration.

Inner tracking (for $r<48 \mathrm{~cm}$ ) is based to a silicon multilayer tracker, which includes LAYER00, SVXII and ISL. The combination of these devices results in up to 
eight points for each fiducial track and covers most of the $p \bar{p}$ interaction region; position measurements ${ }^{1}$ with long lever arms provide $p_{T}$ measurements with a precision up to $\delta p_{T} / p_{T}^{2} \sim 0.4 \%(\mathrm{GeV} / \mathrm{c})^{-1}$ in the silicon system alone.

The potentialities of this architecture can be fully exploited by means of a tracking procedure which integrates the information supplied by various detectors. Several tracking algorithms have been developted: besides the stand-alone COT reconstruction and tracking in the silicon system seeded from COT tracks (inherited from Run I), stand-alone silicon reconstruction and outward extension of silicon tracks in the COT are used [1]. The latter options allow tracking to be extended in the region $1<|\eta|<2$.

\subsubsection{Resolutions}

The contribution of the silicon vertex detector, with an increased numbers of layers and shorter distances of the closest ones from the beam axis, is crucial for pursuing high precision measurements of $d$ and $z_{0}$. The effect of augmenting the number of silicon layers and of the lever arms of the outer layers implies, on the other hand, an enhanced resolution on $p_{T}$ as well. A summary of the tracking resolutions in Run II by CDF is reported in table 4.1 .

\begin{tabular}{||c||c|c||}
\hline \multicolumn{3}{||c||}{ Resolution } \\
& COT & COT+SVXII+ISL \\
\hline$\delta p_{T} / p_{T}^{2}\left[(\mathrm{GeV} / \mathrm{c})^{-1}\right]$ & $3 \times 10^{-3}$ & $1 \times 10^{-3}$ \\
$\delta d[\mu \mathrm{m}]$ & 600 & 30 \\
$\delta z_{0}[\mu \mathrm{m}]$ & $5 \times 10^{3}$ & 30 \\
$\delta \cot \theta$ & $6 \times 10^{-3}$ & $4 \times 10^{-4}$ \\
\hline
\end{tabular}

Table 4.1: Summary of resolutions on various track parameters in Run II.

The determination of the absolute momentum scale can be obtained by means of a comparison between a clean signal and some reference. In the case of CDF the decay $\mathrm{J} / \psi \rightarrow \mu^{+} \mu^{-}$furnishes such a reference; the estimate of the momentum scale is performed by renormalizing the observed $\mathrm{J} / \psi(1 \mathrm{~S})$ peak to the world average.

\subsection{Primary vertex reconstruction}

Reconstructed tracks locate the primary vertex in each event: its $z$ position is then used in defining the actual pseudorapidity of each physics object reconstructed in the event, while its transverse position is important since it provides a benchmark for secondary vertex-finding procedures. The $p \bar{p}$ luminous region has a Gaussian spread of about $35 \mu \mathrm{m}$ in the transverse plane: its location is known with good precision, but a more precise determination can be obtained from the track parameters. Two algorithms can be run on all reconstructed tracks to determine the primary vertex position which then undergoes a significant improvement with respect to the beam spot information alone being also more

\footnotetext{
${ }^{1}$ All layers except the innermost provide both $r-\phi$ and $r-z$ information
} 
reliable on an event-by-event basis. A concise description of the two algorithms follows [2]:

1. PrimVtx. This procedure begins with applying the following cuts to event tracks

- $|d|<1.0 \mathrm{~cm}$;

- $\frac{d}{\sigma}<3.0$;

where $\sigma$ is the error associated to $d$ and $\frac{d}{\sigma}$ is called significance of the impact parameter. Tracks surviving this initial selection are fitted to a common vertex. The residual for each track w.r.t. the common vertex is calculated and a track is dropped from further calculation if $\left|z_{\text {track }}-z_{\text {vertex }}\right|>1.0 \mathrm{~cm}$. Then the vertex fit is calculated again and the procedure is iterated until stability is reached and no tracks have $\chi^{2}>10$ w.r.t. the fitted vertex.

2. ZVertexColl. This algorithm also starts from a track-based vertices satisfying some minimal quality requirements. Among these, there are a lot of fake vertices which are removed by requiring that vertices be associated with a certain number of tracks with $p_{T}>300 \mathrm{MeV} / \mathrm{c}$. A track is associated with a vertex if $\left|z_{\text {track }}-z_{\text {vertex }}\right|<1.0 \mathrm{~cm}$, where $z_{\text {vertex }}$ is calculated using the silicon information only and if $\left|z_{\text {track }}-z_{\text {vertex }}\right|<$ $5.0 \mathrm{~cm}$ when using the COT tracking. The $z$ vertex position is calculated from track $z_{0}$ s weighted with their error $\delta$ :

$$
z=\frac{\sum_{i} \frac{z_{0 i}}{\delta_{i}^{2}}}{\sum_{i} \frac{1}{\delta_{i}^{2}}}
$$

Vertices found with this procedure are classified by quality flags according to the number of tracks with SVXI/COT hits that are associated to the vertex itself. Vertices associated with COT tracks have shown to be less contaminated by fakes. Hence, a higher quality is given to them as illustrated in Tab. 4.2.

\begin{tabular}{|c|c|}
\hline \hline Quality & Vertex type \\
\hline 0 & all vertices \\
4 & $\geq 1$ tracks with COT hits \\
7 & $\geq 6$ tracks with SVXII hits and $\geq 1$ tracks with COT hits \\
12 & $\geq 2$ tracks with COT hits \\
28 & $\geq 4$ tracks with COT hits \\
60 & $\geq 6$ tracks with COT hits \\
\hline \hline
\end{tabular}

Table 4.2: Quality assigned to vertices from the ZVertexColl algorithm.

\subsection{Jets}

Isolated partons emerging from the initial $p \bar{p}$ collision, due of the nature of strongly interacting matter, undergo a process called fragmentation; during fragmentation, a parton shower is developed from the original parton as an effect of the increase of the strong coupling constant with decreasing $p_{T}$. Fragmentation terminates with hadronization, a process where all partons recombine in colour singlet states, corresponding to on shell hadrons. By momentum conservation, the more energetic the initial parton, the closer to 
each other the resulting hadrons are confined in phase-space. These clusters of particles are called jets and their importance is related to the fact that they represent the only physically-measurable quantity carrying a signature of the initial parton.

\subsubsection{Jet Clustering}

At CDF the algorithm mostly used for jet reconstruction is the cone algorithm; the opening angle of the cone is usually defined in terms of a radius in the $\eta-\phi$ plane. Due to the relativistic properties of pseudorapidity, in fact, this definition allows one to conveniently identify jets by circular disks in the $\eta-\phi$ calorimetry plane. The magnitude of the radius $\left(R=\sqrt{\Delta \phi^{2}+\Delta \eta^{2}}\right)$ must be chosen according to the characteristics of the physical process under study. Hence, lower radii (typically 0.4 ) will be preferred in high multiplicity events, where a higher jet resolution is required, while higher values of $R$ (typically 0.7 ) are chosen otherwise.

The first step in jet clustering consists in assigning to each calorimetry tower $(\Delta \eta \simeq 0.1$, $\left.\Delta \phi \simeq 15^{\circ}\right)$ a $r \eta \phi$ vector, which originates at the interaction point and points towards the tower corresponding to the energy barycentre ${ }^{2}$. After the vector assignment, the clustering procedure follows two other steps.

Preclustering. Vectors with $E_{T}>1 \mathrm{GeV}$ are ranked according to a decreasing magnitude order; the vector list is then scanned downwards in the following way:

1. a circle of radius $R$ is drawn around the first element of the list;

2. all vectors falling inside the circle are summed to it and removed from the list;

3. a new circle is drawn around the next vector in the list;

4. steps 2 and 3 are repeated until the end of the list is reached.

Clustering. A second iterative procedure involves all the remaining vectors with $E_{T}>0.1 \mathrm{GeV}$ which are associated to the cone containing them:

5. the $E_{T}$ associated to each circle is recomputed by merging all the enclosed vectors and new cluster barycentres are calculated;

6. new circles are drawn around the barycentres so obtained;

7. steps 5 and 6 are reiterated until a stable configuration ${ }^{3}$ is reached.

It may happen that two circles overlap; in this case, if the total contribution of all vectors belonging to the intersection region does not exceed the $75 \%$ of the magnitude of the smaller of the two resultants, each vector is assigned to the closest resultant. Otherwise, the two circles are replaced by a single one, centered around the sum of their resultants.

The jet 4-momentum is computed assuming that each vector corresponds to a massless particle originated in the primary vertex and detected in the energy barycentre of the

\footnotetext{
${ }^{2}$ The energy barycentre is defined assuming that all electromagnetic and hadronic energies have been released at a depth of 6 radiation lengths $\left(X_{0}\right)$ and 10 interaction lengths $(\lambda)$ respectively.

${ }^{3} \mathrm{~A}$ stable configuration is achieved when circles are reconstructed in the same position for two consecutive iterations; usually this happens after three iterations.
} 
corresponding tower, therefore it is calculated by summing the 4-momenta of all vectors which are included in the cone:

$$
\begin{aligned}
& E=\sum_{i}\left(E_{i}^{\text {had }}+E_{i}^{e m}\right) \\
& p_{x}=\sum_{i}\left(E_{i}^{\text {had }} \sin \theta_{i}^{\text {had }}+E_{i}^{\text {em }} \sin \theta_{i}^{\text {had }}\right) \cos \phi_{i} \\
& p_{y}=\sum_{i}\left(E_{i}^{\text {had }} \sin \theta_{i}^{\text {had }}+E_{i}^{e m} \sin \theta_{i}^{\text {had }}\right) \sin \phi_{i} \\
& p_{z}=\sum_{i}\left(E_{i}^{\text {had }} \cos \theta_{i}^{\text {had }}+E_{i}^{\text {em }} \cos \theta_{i}^{\text {had }}\right)
\end{aligned}
$$

where $\theta_{i}^{\text {had }}$ and $\theta_{i}^{\text {em }}$ correspond to the polar angle associated to the hadronic and electromagnetic section of a calorimetric tower respectively.

\subsubsection{Jet corrections}

According to CDF definition, jets emerge from a vector sum of the momenta associated with calorimeter towers enclosed in a certain region (cone). However, jet energies measured in calorimeters suffer from intrinsic limits due to the calorimeter itself and the jet reconstruction algorithm. For instance, different particles produce different responses and some of them could reach uninstrumented regions of the detector. Moreover the jet clustering does not take into account possible multiple interactions and border effects due to the fixed cone size such as energy partially radiated outside of it. Unambiguous results can be obtained only if adequate corrections ([3],[4],[5]), aimed at reducing systematic effects degrading the energy determination, are applied to the raw energies detected by the calorimeters. These effects have been accounted for in the definition of jet 4-momentum, in order to suitably rescale the transverse energy of each jet to a value which represents the most likely $E_{T}$ that would have been measured if all the degrading effects were not present. The most general form for the corrections to be applied to the $p_{T}^{\text {raw }}$ of a jet (cone $\left.R=\sqrt{\Delta \eta^{2}+\Delta \phi^{2}}\right)$ is the following [3]:

$$
p_{T}(R)=\left(p_{T}^{r a w}(R) \times f_{r e l}-U E M(R)\right) \times f_{a b s}(R)-U E(R)+O O C(R),
$$

where:

$f_{\text {rel }}$ : $\quad$ relative energy scale factor, correcting for non-uniformities in the detector response;

$\operatorname{UEM}(R)$ : correction for multiple interactions;

$f_{a b s}(R)$ : absolute energy scale factor;

$U E(R)$ : correction for underlying event;

$O O C(R)$ : out-of-cone losses correction.

Some more details of each kind of jet correction will be presented in the following:

$\star$ Relative energy scale correction. Imperfect calorimeter coverage as well as limited response for low- $p_{T}$ hadrons, also contributes in degrading energy measurements, 
since little or no energy deposition is detected for particles escaping through detector cracks. This effect leads to a $\eta$-dependent response of the calorimeter depending on the $\eta$ region considered. Relative (or $\eta$-dependent) jet energy corrections are then applied to raw jet energies to correct for these non-uniformities. The calorimeter response in each $\eta$ section $^{4}$ is normalized to the response in the region $0.2 \leq|\eta| \leq$ 0.6 , because this region is far away from detector cracks and it is expected to have a stable response (central calorimeters are better calibrated and understood). The correction factor is obtained using the dijet balancing method applied to dijet events: this method starts selecting events with one out of two jets in the central region $0.2 \leq|\eta| \leq 0.6$. This jet is defined as trigger jet while the second jet is called as probe jet. If both jets are central the trigger and probe jets are equivalent. In a $2 \rightarrow 2$ process, the transverse momentum of the first jet should be equal to the second one. This characteristic is used to calculate the following variable $\Delta p_{T} f$, which is a $p_{T}$ balancing fraction:

$$
\Delta p_{T} f=\frac{p_{T}^{\text {probe }}-p_{T}^{\text {trigger }}}{\left(p_{T}^{\text {probe }}+p_{T}^{\text {trigger }}\right) / 2}=\frac{\Delta p_{T}}{\left(p_{T}^{\text {probe }}+p_{T}^{\text {trigger }}\right) / 2}
$$

Then the correction factor $f_{\text {rel }}$ in order to balance the probe jet energy scale to the trigger one is calculated:

$$
f_{\text {rel }}=\frac{p_{T}^{\text {probe }}}{p_{T}^{\text {trigger }}}=\frac{2+\Delta p_{T} f}{2-\Delta p_{T} f}
$$

* Multiple interactions correction. A multiple interaction occurs when two or more collisions take place during the same beam crossing; in this case more events are overlapped and the extra production of particles can influence the jet definition. A correction for this effect is extracted using a sample of minimum bias events ${ }^{5}$ : for each event the average transverse energy $E_{T}$ inside cones of different radii is measured in a region far away from cracks $(0.1 \leq|\eta| \leq 0.7)$; hence the distribution of the average $E_{T}$ as a function of the number of quality 12 vertices (see Tab. 4.2) is fitted with a straight line and the slope $\left(f_{U E M}\right)$ is extracted. For each cone size, $f_{U E M}$ is the energy that needs to be removed from the raw jet $E_{T}$ when more than one vertex is seen in a jet event. The multiple interaction correction has the form:

$$
U E M(R)=f_{U E M}\left(N_{v t x}-1\right)
$$

where $N_{v t x}$ is the number of quality 12 vertices in the event.

* Absolute energy scale correction. A jet is in general composed of different types of particles with a wide momentum spectrum. The calorimeter response to a particle

\footnotetext{
${ }^{4} \eta$ sections are determined by the granularity of the calorimeter.

${ }^{5} \mathrm{~A}$ minimum bias event is theoretically defined as the inelastic part of an interaction which includes the whole spectrum of non-diffractive processes. In a hadron-hadron interaction the minimum bias cross section corresponds to roughly the $80 \%$ of the total inelastic one.
} 
depends on its momentum which is in general different from its momentum measured at calorimeter level. The absolute scale correction converts the calorimeter cluster transverse momentum to the sum of transverse momenta of the particles in the jet cone: calorimeter energy is converted in particle energy based on a calorimeterto-hadron matching procedure. After this correction the energy scale of a jet is assumed to be independent of the CDF detector. The correction factor is extracted in the following way. First, a sample of MC events is generated with the full CDF simulation. Then a list of clusters of calorimeters towers and of HEPG particles is compiled by means of the standard cone cluster algorithm. Once clusters are identified, jets at calorimeter level are associated with jets at hadron level. The probability of measuring a jet $p_{T}^{c a l}$ given a jet with fixed value of $p_{T}^{\text {had }}$ is taken as correction factor $f_{a b s}(R)$ which also depends on the cone size.

* Underlying event correction. The underlying event, originating mostly from soft spectator interactions, is an additional effect which needs to be taken into account when considering jet production in a hadron-hadron collision. In fact, the spectator interactions may be hard enough to produce soft jets so that energy from the underlying event could carry an extra contribution to the jet cones of the hard scattering process thereby biasing the energy measurement. A correction factor has been calculated using a sample of minimum bias events selecting events with only one good reconstructed vertex in order to avoid possible overlaps with multiple interaction events. For each event, the $E_{T}$ in different cone sizes has been measured in a region far away from cracks $(0.1 \leq|\eta| \leq 0.7)$ and the correction $U E(R)$ is extracted from the mean values of the $E_{T}$ distribution.

$\star$ Out-of-cone correction. The cone clustering procedure has a finite resolution essentially imposed by $R$; particles which, during hadronization or subsequent hadron decay, are emitted at large angles, may not be included in the jet they would naturally belong to. These out-of-cone losses could be also caused by low- $p_{T}$ particles being trapped in the magnetic field. This energy difference between the parton $E_{T}$ and the corresponding hadron cluster must be added to the jet to recover the energy at parton level. This correction factor is extracted using dijet events. The method used is similar to the one used for the estimate of the absolute energy correction. Hadron-level jets are matched to partons and, from the difference in energy between the two, the correction factor $O O C(R)$ is extracted .

Depending on the physics analysis, all the jet corrections or just a subset of them are applied.

\subsection{Leptons}

Detection and tagging of leptons is crucial at hadron colliders, where they provide powerful means for studying electroweak processes and, in general, final states characterized by the presence of one or more leptons. According to their production mode, leptons can 
be broadly divided into two classes: prompt high $p_{T}$ leptons, mainly coming from vector boson decay $\left(\mathrm{Z}^{0} \rightarrow \ell^{+} \ell^{-}\right.$and $\left.\mathrm{W}^{ \pm} \rightarrow \ell^{ \pm} \nu_{\ell}\right)$ or from new physics processes, and non-isolated leptons. The latter, referring to leptons embedded within a jet, are usually characterized by a softer $p_{T}$ spectrum and can be related to the semileptonic decay of charmed or bottom hadrons. The search presented in this work focuses on the study of medium-high $p_{T}$ leptons $\left(p_{T}>4 \mathrm{GeV} / \mathrm{c}\right)$, in particular electrons and muons, which are the products of the associated production of chargino and neutralino in the context of the supersymmetric theory. A description of how an electron and a muon are identified in terms of quantities measured by the components of the $\mathrm{CDF}$ detector is presented in the following.

\subsubsection{Electrons}

Electron tagging [6] at CDF generally relies on energy depositions in the electromagnetic towers of the calorimeter and on the reconstructed tracks in the event but the identification procedures depend on both the transverse energy and the rapidity of the candidate. In general an electron candidate is defined by

$\diamond$ a cluster in the electromagnetic calorimeter;

$\diamond$ one or more tracks matched to the cluster;

$\diamond$ shower max and preshower clusters associated with the calorimeter cluster.

After all electron candidates are found in the event, in order to increase the purity of the sample, a set of quality cuts is imposed on several variables characterizing each electron candidate. The quantities used for the selection are referred to as identification variables.

The electromagnetic clustering procedure. Up to July 2001 a common electromagnetic (EM) clustering algorithm had been used both for the central and the plug calorimeters, which produced, by default, $3 \times 3$ tower clusters. This was deemed too large for the plug giving excessive extraneous energy in the outer towers of the clusters. Since July 2001 a new algorithm for the forward region has been introduced as default which limited the cluster size to $2 \times 2$, i.e. a cluster is composed of a seed tower and at most three adjacent daughter towers. A concise description of the clustering procedure is given in the following with some details regarding the differences between the central and plug algorithms.

The electromagnetic clustering needs the following input information:

$\star$ the $z$ vertex of the event which is by default fixed at $z=0.0$;

$\star$ a list of towers with $E_{T}>100 \mathrm{MeV}$, which are defined as valid seed towers for clustering;

* a second list of seed towers having $E_{T}>2 \mathrm{GeV}$.

Before the real clustering begins the seed towers are sorted according to descending EM transverse energy and the clustering procedure starts with the first seed tower of the list. The procedure is applied iteratively for all seed towers checking that each seed tower considered is not already included in a cluster, in order to prevent towers from being used in more than one cluster. When the clustering algorithm ends a check of the global properties of each cluster is made; the default requirements are $E_{T}>2 \mathrm{GeV}$ and $E_{\text {had }} / E_{\text {em }}<0.125$. Other details about the clustering algorithms are: 
$\diamond$ The CEM algorithm. For each seed tower, the clustering algorithm provides a list of neighbor towers that share a $\eta$ or $\phi$ boundary or touch the tower at one of its four corners. The method then iterates through this list and determines whether a tower should be included in the cluster by requiring that the tower EM or hadron energy have non-zero value thus assuring a sensible hadron/EM energy ratio.

$\diamond$ The PEM algorithm. As in the CEM, the plug algorithm provides a list of towers that shares a border or a corner with the seed one. The algorithm then finds the largest EM $E_{T}$ tower that shares a border with the seed tower. If $\mathrm{EM} E_{T}^{\text {tower }}>E_{T}^{\text {min }}$ and $E_{T}^{\text {tower }} / E_{T}^{\text {seed }}>$ Ratio, $E_{T}^{\text {min }}$ and Ratio being settable thresholds (default values are $100 \mathrm{MeV}$ and 0 respectively) this tower is designated as the daughter tower. Next, the method searches for a pair of towers not including the daughter: it sums the EM $E_{T}$ of each pair of towers selecting the pair with the largest $E_{T}$. This pair must have EM $E_{T}>E_{T}^{\text {min }}$ and $E_{T}^{\text {pair }} / E_{T}^{\text {seed+daughter }}>$ Ratio, $E_{T}^{\text {min }}$ and Ratio being as defined above.

Track association. When the clustering in the electromagnetic calorimeter ends, for each identified cluster a matching algorithm loops over the collection of tracks in order to associate one or more tracks with an EM cluster. Each track of the event is iteratively extrapolated to the plane of the CES for a CEM cluster, or the PES for a PEM cluster. The association criteria demand that the track actually transverses a CEM tower in the wedge of interest, while in the PEM a track is associated to the EM cluster if it intersects the seed tower or one of the towers that have a common border or corner with the seed one.

Shower max and preshower clusters association. In the central calorimeter there are two CES cluster collections. An unbiased collection that is created from a list of wire or strip seeds over a threshold energy and a track based collection that uses the wire or strip nearest an extrapolated track as a seed. Association of a CES cluster of the unbiased collection with a CEM cluster only requires the two clusters to be in the same wedge. If the CES cluster is from the track based collection, it is associated with the CEM cluster if, in addition, the track seeding the cluster is one of those previously associated tracks to the CEM cluster. In the PES there is a single collection created using strips over a certain energy threshold as seeds. The PES cluster is associated to a PEM cluster if the difference between the PES and PEM $\Delta r=\sqrt{\Delta x^{2}+\Delta y^{2}}$ is less than $10 \mathrm{~cm}$, where $x$ and $y$ are the global CDF $x y$ coordinates. In general after the association there are more than one shower max cluster associated to the calorimeter cluster.

The last objects associated to the calorimeter cluster are preshower clusters: the matching procedure is similar to that for shower max clusters. Currently only the central pre-radiator detector is handled.

Identification variables. As described above, an electron is essentially one (or more) reconstructed tracks extrapolating to an electromagnetic deposit. After all electron candidates are identified in the event a set of quality requirements are applied on some measurable quantities of each candidate. First it is required that all electrons be in the $E_{T}$ and $p_{T}$ range of interest, where $E_{T}$ is the electromagnetic transverse energy associated to the EM cluster and $p_{T}$ is the transverse momentum of the track with maximum transverse momentum associated to the cluster $\left(\max -p_{T}\right.$ track $)$. The max- $p_{T}$ track undergoes 
some specific cuts that garantee its quality: it must have a $z$ vertex reasonably close to the nominal origin of CDF and a minimum number of COT hits on both axial and stereo superlayers. The candidate must also be consistent with the $E / p$ electron hypothesis ${ }^{6}$. The possibility of a non-electron track faking the previous requirements because of the contribution of surrounding soft particles releasing energy in the same calorimeter towers is still high; a further contribution may also come from isolated hadrons performing early showering (i.e., before reaching the hadronic section of the calorimeter). Two sets of requirements help in keeping these effects under control; the first one relies on two purely calorimetric variables:

$\diamond E_{h a d} / E_{e m}$ : an upper bound on this variable results in an upper bound on the hadronic energy deposition detected within a cluster cone. According to the process that led to the electron, the ratio $E_{\text {had }} / E_{e m}$ can be computed from single towers or multiple tower clusters. As explained in the previous part of this section, this requirement is also applied at the EM clustering stage to avoid, before any further identification, clusters with a too large hadronic energy fraction.

$\diamond L_{s h r}$ : from a comparison of the observed calorimeter lateral shower profile to that of test-beam electrons, $L_{s h r}$ is required to be less than a upper limit.

The second set, on the other hand, exploits the information provided by the CES detector. For this reason the electron candidate must be also fiducial inside the volume of this detector. The CES proves to be of crucial importance for tagging electrons, due to its finer granularity with respect to the calorimeter towers. Two kinds of information, accessible after CES clustering, are particularly useful for electron identification:

position: the centroids of the clusters reconstructed in the strip and wire planes provide a $x-z$ reading in the local wedge-coordinate system of the shower max detector. This information is useful for providing a high quality track-cluster matching imposing for instance an upper bound on the difference $\Delta z$ (similarly for $\Delta x$ ) between the local $z$ of the CES cluster and the $z$ position of the max- $p_{T}$ track extrapolated at the CES surface;

shape: two $\chi$-squared comparisons $\left(\chi_{\text {strip }}^{2}, \chi_{\text {wire }}^{2}\right)$ of CES cluster profile fits (in strip and wire planes respectively) to test beam electrons.

Due to the different setup of the detector in the plug, the information available leads to different identification variables with respect to the central region. As far as the PES information is concerned, the goodness of the fit $\left(\chi_{P E M}^{2}\right)$ obtained when comparing the center of the EM shower to test beam measurements helps in improving the plug electron identification. Moreover, the shower max detector information is only used to assure that most of the energy deposition is concentrated in the central part of the PES cluster (PES $5 / 9)$.

Finally, electrons (both central and plug) of interest for this analysis are expected to be isolated; therefore, for each candidate, the ratio $E_{T}^{0.4} / E_{T}$ is required to be less than a maximum value, where $E_{T}^{0.4}$ is the transverse energy around the electron cluster in a 0.4 cone after removing the $E_{T}$ of the cluster itself. The set of identification cuts (studied in [7] [8]) applied to each electron candidate is listed in Table 4.3- 4.4. 


\begin{tabular}{|c|}
\hline \hline Central Electron ID Cuts \\
\hline \hline$E_{T} \geq 4 \mathrm{GeV}$ \\
At least one track associated with the EM cluster \\
$E_{\text {had }} / E_{\text {em }} \leq 0.055+0.00045 \times E_{\text {em }} / \mathrm{GeV}$ \\
$E / p \leq 2$ if track $p_{T}<50 \mathrm{GeV} / \mathrm{c}$ \\
$L_{S h r} \leq 0.2$ \\
$\chi_{\text {strip }}^{2} \leq 10$ \\
$-3<Q \times \Delta<1.5 \mathrm{~cm}$ \\
$|\Delta z| \leq 3 \mathrm{~cm}$ \\
COT Axial Segments (with $5 \mathrm{Hits}) \geq 3$ \\
COT Stereo Segments (with $5 \mathrm{Hits}) \geq 2$ \\
$E_{T}^{0.4} / E_{T} \leq 0.1$ \\
$z_{\text {vertex }} \leq 60 \mathrm{~cm}$ \\
\hline \hline
\end{tabular}

Table 4.3: List of the central electron identification cuts.

\begin{tabular}{|c|}
\hline \hline Plug Electron ID Cuts \\
\hline \hline$E_{T} \geq 4 \mathrm{GeV}$ \\
At least one track associated with the EM cluster \\
$1.2 \leq|\eta| \leq 2.0 E_{\text {had }} / E_{\text {em }} \leq 0.05$ \\
$E / p \leq 3$ \\
$E_{T}^{0.4} / E_{T} \leq 0.1$ \\
$z_{\text {vertex }} \leq 60 \mathrm{~cm}$ \\
$\chi_{P E M}^{2} \leq 10$ \\
PES $5 / 9 \geq 0.65$ \\
\hline \hline
\end{tabular}

Table 4.4: List of the plug electron identification cuts.

Conversion removal. A serious source of background is due to photons, arising from the decay of neutral pions, which tend to convert into electron-positron pairs in detector material and need to be removed. This can be achieved by means of an algorithm [9] which, after reconstructing all possible conversion pairs within an event, discards any electron candidate that can be associated to one leg of a conversion pair. In order to tag conversion pairs, the algorithm performs a first selection on the basis of geometrical considerations: two variables, $\Delta \cot \theta$ and $\Delta S$, where $\Delta S$ is the separation of the two legs in the CDF $x-y$ plane, are used to select tracks compatible with a common vertex ${ }^{7}$, which is constrained to be within $50 \mathrm{~cm}$ of the beamline (where the highest concentration of material in the tracking volume is found). Then, two tracks fulfilling this requirement are tagged as a conversion pair.

\footnotetext{
${ }^{6}$ Prompt electrons have energies is in the $\mathrm{GeV}$ scale, so that they are ultra-relativistic particles, hence $E=\sqrt{m^{2} c^{4}+p^{2} c^{2}} \sim p c$.

${ }^{7}$ Actual requirements on these variables are $|\Delta \cot \theta|<0.04$ and $\Delta S<0.2 \mathrm{~cm}$.
} 


\subsubsection{Muons}

The usual method for tagging muons consists in extrapolating a track to the muon detector system and matching it to a stub reconstructed therein [10]. Tagging in the case of muons is much less problematic than for electrons, since the natural tendency of muons to penetrate thick layers of material can be exploited to range out other particles. For this purpose, the shielding offered by the calorimeters is used and detection of charged particles beyond them is achieved by means of the muon detector system. The different subsystems of the muon detector system produce different kinds of information. The chambers measure drift times which are converted to drift distances in the plane perpendicular to the chamber sense wires. Moreover the CMU and the IMU have the means to measure the position of the muon along the sense wire, while the CMP and CMX do not. Different algorithms are used according to the information available in each chamber subsystem.

A fundamental step for muon identification is extrapolating all the tracks to the surface of the muon chambers. Hits in the muon chambers are fitted to linear track segments known as stubs characterized by a stub position and a stub direction. The direction is a unit vector that points from the stub position point in the direction of the muon's flight, so the position and direction together define the line segment. When stubs finding ends, a track-stub matching is performed which identifies tracks for further selection. In general, muons are classified according to the chamber they are detected in: CMUP for muon tracks traversing both $\mathrm{CMU}$ and $\mathrm{CMP}$ volumes, CMU/CMP for tracks detected in the $\mathrm{CMU} / \mathrm{CMP}$

volume only, while escaping the coverage of $\mathrm{CMP} / \mathrm{CMU}$, and CMX for tracks falling in the CMX fiducial volume.

After muon detection, as is done for electrons, further identification cuts are applied to all muon candidates in order to increase the purity of the selection. In addition to applying upper bounds on the muon calorimeter energy deposition consistently with a miminum ionizing particle, quality requirements on the track are imposed. Two variables are used for estimating the goodness of track-stub matching: $\Delta x$, which refers to the distance between the extrapolated track and the stub in the transverse plane at the inner radius of the muon detector, and $\chi^{2}$, which represents the quality of the track-muon match. It is also possible to have a muon with no stubs associated at all (CMIO) which might be still interesting for some physics analyses. In Table 4.5, 4.6, 4.7 cuts used for the identification of muons are listed [11],[12].

The major source of contamination in the case of muons arises from calorimeter punchthroughs (secondary charged pion leakages through the outermost layer of the hadron calorimeter) and from muons produced in the decays in flight of kaons or pions, which are responsible of 'fake' stubs. The former is strongly limited by the coincidence of CMU and CMP in the CMUP category.

Cosmic removal. Another serious background to keep under control consists of cosmic muons. Cosmic rays constantly hitting the atmosphere create showers of partices which often decay to muons leading to a flux of muons through the detector. These muons can leave a nearly straight track (mimicking a very energetic $\mu^{+} \mu^{-}$pair) in the COT with an arbitrary impact parameter depending on where the muon entered the detector. This is the main motivation of the cut on the impact parameter $d_{0}$ listed in Table 4.5 4.6 4.7. From the detector's point of view, the remaining cosmics manifest themself as dimuon 


\begin{tabular}{c} 
High $p_{T}$ muon ID cuts \\
\hline \hline$p_{T} \geq 20 \mathrm{GeV} / \mathrm{c}$ \\
$|\eta| \leq 1.0$ \\
CMUP or CMX Stub \\
$\left|\Delta x_{C M U}\right| \leq 3 \mathrm{~cm}$ \\
$\left|\Delta x_{C M P}\right| \leq 5 \mathrm{~cm}$ \\
$\left|\Delta x_{C M X}\right| \leq 6 \mathrm{~cm}$ \\
$E_{\text {em }} \leq 2+\max \left(0,\left(p_{T}-100\right) \times 0.0115\right) \mathrm{GeV}$ \\
$E_{\text {had }} \leq 6+\max \left(0,\left(p_{T}-100\right) \times 0.028\right) \mathrm{GeV}$ \\
COT Axial Segments (with $5 \mathrm{Hits}) \geq 3$ \\
COT Stereo Segments (with $5 \mathrm{Hits}) \geq 2$ \\
$E_{T}^{0.4} / E_{T} \leq 0.1$ \\
Impact parameter $\left|d_{0}\right| \leq 0.2 \mathrm{~cm}$, if Silicon hits $\left|d_{0}\right| \leq 0.02 \mathrm{~cm}$ \\
$z_{\text {vertex }} \leq 60 \mathrm{~cm}$ \\
\hline \hline
\end{tabular}

Table 4.5: List of high $p_{T}$ muon identification cuts.

\begin{tabular}{c}
\hline \hline Medium $p_{T}$ muon ID Cuts \\
\hline \hline $4 \leq p_{T} \leq 20 \mathrm{GeV} / \mathrm{c}$ \\
$|\eta| \leq 1.0$ \\
CMUP or CMX Stub \\
$\left|\Delta x_{C M U}\right| \leq 3 \mathrm{~cm}$ or $\chi^{2} \leq 9$ \\
$\left|\Delta x_{C M U}\right| \leq 5 \mathrm{~cm}$ or $\chi^{2} \leq 9$ \\
$\left|\Delta x_{C M U}\right| \leq 6 \mathrm{~cm}$ or $\chi^{2} \leq 9$ \\
$E_{\text {em }} \leq 2$ \\
$E_{\text {had }} \leq \frac{p_{T}}{8}+3.5 \mathrm{GeV}$ \\
COT Axial Segments $($ with $5 \mathrm{Hits}) \geq 3$ \\
COT Stereo Segments $($ with $5 \mathrm{Hits}) \geq 2$ \\
$E_{T}^{0.4} / E_{T} \leq 0.1$ \\
Impact parameter $\left|d_{0}\right| \leq 0.2 \mathrm{~cm}$ \\
$z_{\text {vertex }} \leq 60 \mathrm{~cm}$ \\
\hline \hline
\end{tabular}

Table 4.6: List of medium $p_{T}$ muon identification cuts.

events with tracks that are mostly back-to-back. These events are characterized by strange timing of track hits in the COT: these times show a difference roughly equal to the speedof-light transit time from one side to the other of the calorimeter and generally they are not in coincidence with the bunch crossing. In addition to the geometrical back-to-back topology, the timing information coming from the time of flight detector is the main input to the cosmic removal algorithm [13] developed at CDF. 


\begin{tabular}{|c|}
\hline \hline CMIO muon ID Cuts \\
\hline \hline$p_{T} \leq 10 \mathrm{GeV} / \mathrm{c}$ \\
Non-Fiducial CMUP and CMX \\
$E_{\text {em }}$ as for stub muons \\
$E_{\text {had }}$ as for stub muons \\
$E_{\text {tot }}>0.1$ \\
COT Axial Segments (with 5 Hits) $\geq 3$ \\
COT Stereo Segments (with 5 Hits) $\geq 3$ \\
$E_{T}^{0.4} / E_{T} \leq 0.1$ \\
Impact parameter $\left|d_{0}\right|$ as for stub muons \\
$z_{\text {vertex }} \leq 60 \mathrm{~cm}$ \\
\hline \hline
\end{tabular}

Table 4.7: List of stubless muon identification cuts.

\subsection{Missing energy}

The total detected energy in the calorimeters does not necessarily balance the energy sum of the proton and antiproton energies due to possible mismeasurements in the calorimeter itself or due to particles escaping detection such as neutrinos. As many particles escape down the beam pipe it is not possible to apply total momentum conservation, but it is possible to apply transverse momentum conservation assuming that particles escaping down the beam pipe have negligible transverse momentum. If the vector sum of transverse momenta of detected particles is signicantly different from zero the discrepancy can be interpreted in terms of the presence of one or more undetected particles. Therefore the presence of these particles can be inferred from trasverse momentum conservation by introducing a variable called missing transverse energy $\mathbb{E}_{T}([14])$ defined as:

$$
\not_{T} \equiv \sqrt{\mathscr{E}_{T x}^{2}+\mathbb{E}_{T y}^{2}}
$$

where

$$
\begin{aligned}
& \mathscr{E}_{T x} \equiv-\sum_{i=1}^{N_{\text {towers }}} E_{x}^{i} \\
& \mathscr{E}_{T y} \equiv-\sum_{i=1}^{N_{\text {towers }}} E_{y}^{i}
\end{aligned}
$$

where $E_{x}^{i}$ and $E_{y}^{i}$ are the energies of a tower projected along the $x$ and $y$ axis of the CDF cartesian system. The sum runs over all towers of the detector above a certain energy threshold, where the tower energy threshold depends on which region of the detector the tower belongs to.

Missing energy corrections. The missing transverse energy discussed so far is referred to as the raw missing energy. In fact, the $\mathbb{E}_{T}$ directly obtained from the vector sum of the energy deposited in the calorimeter towers needs additional corrections in order to account for the applied jet corrections and for the presence of identified muons in the event. The jet corrections lead to the following correction to the value of the missing 
energy:

$$
\begin{gathered}
\underset{T x}{E} \operatorname{corr}=\mathbb{E}_{T x}^{r a w}+\sum_{j=1}^{N_{\text {jets }}}\left(E_{x, j}^{r a w}-E_{x, j}^{c o r r}\right) \\
\mathbb{E}_{T y}^{c o r r}=\mathbb{E}_{T y}^{r a w}+\sum_{j=1}^{N_{j e t s}}\left(E_{y, j}^{r a w}-E_{y, j}^{c o r r}\right)
\end{gathered}
$$

where the contribution of jets to the absolute value of $\mathbb{E}_{T}$ becomes the corrected energy of each jet considered in the event. The correction for muons is performed by treating muons as minimal ionizing particles, i.e. particles with tracks with a measured $p_{T}$ but minimal energy deposit in the calorimeter. The correction to the missing energy consists in a vectorial subtraction of the transverse momentum of the muon track from the raw $\mathbb{E}_{T}$ adding the corresponding deposit of total energy of the muon measured by the calorimeter:

$$
\begin{aligned}
& \mathscr{E}_{T x}^{c o r r}=\mathbb{E}_{T x}^{\text {raw }}-\sum_{j=1}^{N_{\text {muons }}}\left(p_{x, j}-E_{x, j}^{e m+h a d}\right) \\
& \mathscr{E}_{T y}^{c o r r}=\mathbb{E}_{T y}^{r a w}-\sum_{j=1}^{N_{\text {muons }}}\left(p_{y, j}-E_{y, j}^{e m+h a d}\right)
\end{aligned}
$$

where the sum runs over all the identified muons in the event. 


\section{Bibliography}

[1] P. Gatti Performance of the new tracking system at CDF II, PhD thesis, 2000.

[2] J.F. Arguin et Al. The $z$-Vertex Algorithm in Run II , CDF internal note 6238, 2002.

[3] CDF Collaboration Jet Energy Correction at CDF , CDF internal note 7543, 2005.

[4] A.A. Bhatti et Al. Update on relative jet energy corrections, CDF internal note 7354, 2005.

[5] B. Cooper et Al. Multiple interaction corrections, CDF internal note 7365, 2004.

[6] R. Wagner Electron Identification for Run II: Algorithms , CDF internal note 5456, Mar 2003.

[7] C. Hill, J. Incandela, C. Mills Electron Identification in Offline Release 5.3 , CDF internal note 7309, Nov 2005.

[8] A. Attal ID Efficiencies for Medium $E_{T}$ Plug Electrons, CDF internal note 7345, Aug 2005.

[9] E. Halkiadakis et Al. A Conversion Removal Algorithm for the 2003 Winter Conferences, CDF internal note 6250, Jan 2003.

[10] J. Bellinger et Al. A Guide to Muon Reconstruction and Software for Run 2 , CDF internal note 5870, Nov 2002.

[11] D. Bortoletto ID efficiency for intermadiate $p_{T}$ muons , CDF internal note 7197, Aug 2004.

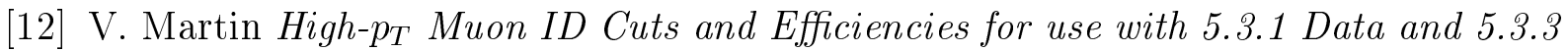
$M C$, CDF internal note 7367, Nov 2004.

[13] A. Taffard Run II Cosmic ray Tagger, CDF internal note 6100, Aug 2002.

[14] CDF MET working group webpage http://wwwcdf.fnal.gov/internal/physics/exotics/susy/r2a/met . 



\section{Chapter 5}

\section{Analysis signature and data samples}

In the first part of this chapter the associated chargino-neutralino production is discussed illustrating some more details of the trilepton channel. This leads up to the description and the motivation of the analysis strategy. In the second part the analysis approach will be presented; in particular the blind analysis strategy is described and motivated. The data set and Monte Carlo samples are then described in the following two sections. The last part of this chapter is dedicated to the procedure used to account for the background contribution coming from misidentified leptons.

\subsection{The analysis signature}

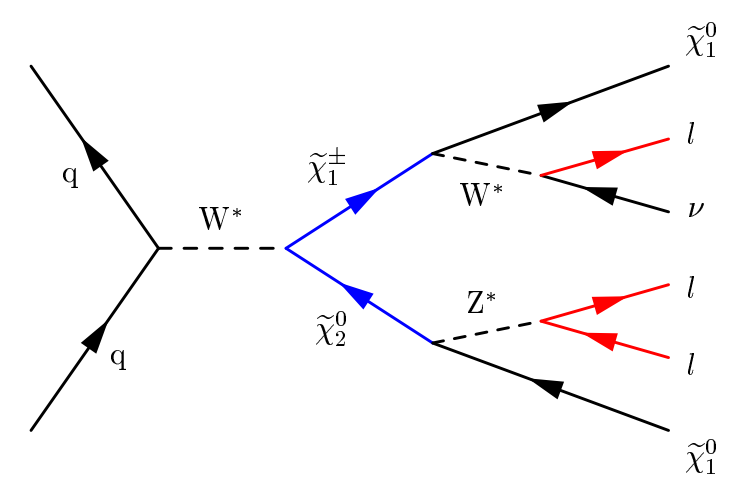

Figure 5.1: Chargino and neutralino associated production decaying via gauge bosons into trilepton final state.

As described in Chapter 1 supersymmetry is a possible extension of the Standard Model and the minimal supergravity model mSUGRA is one of its possible scenarios. This scenario, incorporating $R$-parity conservation, is assumed in this thesis to search for the supersymmetric partners of the gauge and Higgs bosons $(\tilde{\chi})$. In particular the analysis aims at studying the associated production of chargino and neutralino as predicted by SUSY with subsequent decay into leptons. 


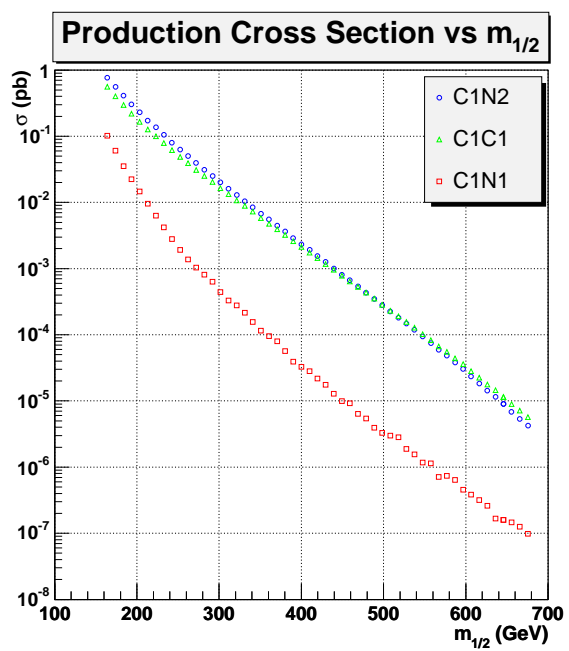

Figure 5.2: Production cross sections of supersymmetric processes with a center of mass energy $\sim 2 \mathrm{TeV}$ (C1N2 stands for $\chi_{1}^{ \pm} \chi_{2}^{0}$ and C1C1 C1N1 can be similarly interpreted).

The $\tilde{\chi}$ family includes 4 neutralinos $\tilde{\chi}_{1,2,3,4}^{0}$ and two charginos $\tilde{\chi}_{1,2}^{ \pm}$: the numerical subscript is related to the mass hierarchy i.e. $\tilde{\chi}_{1}^{0}$ and $\tilde{\chi}_{4}^{0}$ are respectively the lighest and the heaviest neutralino and similarly for charginos. Assuming that $\tilde{\chi}_{1}^{0}$ is the LSP and considering the predicted leptonic decays of chargino and neutralino

- $\tilde{\chi}_{2}^{0} \rightarrow \tilde{\chi}_{1}^{0}+l^{+} l^{-}$

- $\tilde{\chi}_{1}^{ \pm} \rightarrow \tilde{\chi}_{1}^{0}+l^{ \pm} \nu_{l}$

there are several possible $\tilde{\chi} \tilde{\chi}$ pairs that can be considered:

1. the $\tilde{\chi}_{1}^{ \pm} \tilde{\chi}_{1}^{0}$ pair, composed of the lowest mass particles, is overwhelmed by the leptonic decay of $\mathrm{W}^{ \pm} \rightarrow \ell^{ \pm} \nu_{\ell}$,

2. the $\tilde{\chi}_{1}^{ \pm} \tilde{\chi}_{1}^{ \pm}$pair also suffers from a high background coming from WW and ZZ production,

3. the $\tilde{\chi}_{1}^{ \pm} \tilde{\chi}_{2}^{0}$, although still subject to high SM electroweak background, has the highest pair production cross section as shown in Fig. 5.2.

This analysis focuses on the $\tilde{\chi}_{1}^{ \pm} \tilde{\chi}_{2}^{0}$ associated production. The Drell-Yan process $\left(\mathrm{Z} / \gamma^{\star} \rightarrow l^{+} l^{-}\right)$, which is one of the highest SM backgrounds, can be reduced by excluding the appropriate mass window. By exploiting the high hadronic activity and the non isolated leptons associated with the heavy flavour contributions $(b \bar{b} / c \bar{c}, t \bar{t})$, they can also be controlled and separated from the SUSY signal.

As illustrated in 1.3.4 chargino-neutralino production occurs in a $p \bar{p}$ collision via a virtual squark (t-channel) or a virtual $\mathrm{W}$ (s-channel) (the latter Feynman diagram is reproduced in Fig. 5.1). The total $\tilde{\chi}_{1}^{ \pm} \tilde{\chi}_{2}^{0}$ production in the trilepton mode is the result of the interference of these two amplitudes. It follows that, the final state includes three isolated leptons and missing energy coming from the neutrino and the LSPs. The $p_{T}$ range of the leptons depends on the difference between the $\tilde{\chi}_{2}^{0}$ and $\tilde{\chi}_{1}^{0}$ mass: the smaller this mass difference the lower the $p_{T}$ of the final state leptons. 


\subsection{A blind analysis}

A blind analysis [1][2] is an analysis in which the final result and the data on which it is based are kept hidden from the analyst until the analysis is essentially complete. The main motivation is to avoid experimenter's (subconcious) bias. It is worth emphasising that the value of a measurement does not contain any information about its correctness. So knowledge of its value is of no use in performing the analysis itself. This is even more important when new physics is searched in order not to be biased by the model predictions. The approach for a blind analysis in general depends on the specific type of analysis. Typically one uses Monte Carlo to simulate the signal and characterize the backgrounds. Blind analysis of a counting experiment means optimizing all the cuts using such samples before looking at the signal data.

The analysis described here regards a counting experiment and the general approach can be summirized as follows. First, the kinematic region where we expect the SUSY signal (signal box) is not directly investigated. Control regions outside the signal box are defined in order to test different parts of the phase space separately. These control regions are kinematic regions where the new physics signal is expected to be negligible and here data are compared to the Standard Model Monte Carlo predictions. Once the Monte Carlo describes the behaviour of the data in the control regions, both with respect to the shape of the distributions and number of events in a satisfactory manner, a maximization of the signal sensitivity is performed using MC samples. This maximization of the signal over background ratio consists in identifying useful discriminating variables. After the SM backgrounds are understood in the control regions and the predicted signal sensitivity has been maximized, the next step of the analysis is referred to as opening the box i.e. looking into the signal region and comparing number of expected (background+signal Monte Carlo) and observed (data) events.

\subsection{The Data set}

Because this analysis searches for events characterized by three leptons of relatively low $p_{T}$ and some missing energy, the ideal trigger path for collecting events would have been an inclusive low $p_{T}$ single lepton trigger. However this could not be allowed in CDF Run II because the large bandwidth of such a trigger would have excluded triggers for other analyses. The compromise between bandwidth limitations and the need to lower the $p_{T}$ threshold of leptons was a trigger which collects events with two leptons. This trigger was designed specifically for supersymmetric searches and thus called the SUSY DILEPTON trigger [3][4]. Details of the trigger are given in Chapter 3.2.

The behaviour of a trigger must be stable in time in order to guarantee the quality of the collected events. For this reasons, a preliminary study of the stability of the SUSY DILEPTON trigger was performed and is presented in Appendix A. The SUSY DILEPTON trigger is composed of trigger paths which require two electrons, two muons or one electron and one muon with $p_{T} \geq 4 \mathrm{GeV} / \mathrm{c}$ (see Tab. 3.3).

The analysis presented in this work focuses on trilepton final states characterized by the presence of an electron and a muon plus an additional third lepton. The trigger paths that could be used for the $e \mu$ channel are: CEM4_CMU4, CEM4_CMUP4, CEM4_CMX4, CMU4_PEM8, CMUP4_PEM8, CMX4_PEM8. To begin with, events from CEM4_CMUP4 
trigger path were chosen. This trigger requires a central electron CEM 4 and a central muon CMUP4 where central means $|\eta|<1.1$. This thesis restricts itself to the analysis of events from this path.

The $e \mu$ channel while being particularly clean of standard model backgrounds such as the $\mathrm{Z}, \mathrm{Y}, \mathrm{J} / \psi$ peaks, suffers from very low statistics. For this reason two other samples have been used as high statistics control regions to gain confidence in the lepton reconstruction and the ability to reproduce backgrounds: the data collected by DIELECTRON_CENTRAL4 and DIMUON_CMUPCMUP4 triggers which are respectively a dielectron and a dimuon samples.

\subsubsection{Luminosity}

The data are collected by the CDF detector in "runs" which are periods of data taking which can vary from a few minutes up to $\sim 30$ hours. Depending on the conditions of the detector, data collected in some runs could be marked as bad for having portions of the detector turned off or bad beam conditions or the trigger not functioning correctly. Runs are considered good if, in addition to the calorimeter, the COT and the trigger, the muon detector components are marked as good. After the subtraction of bad runs, the total integrated luminosity used for the analysis described in this thesis is $\int L d t=224 \pm 13 \mathrm{pb}^{-1}$ which corresponds to the data taken between March 2002 and February 2004.

\subsection{Monte Carlo samples}

The Monte Carlo technique has been used to simulate large ensembles of events for all interesting physics processes, both signal and background. Using these events the distributions of variables describing the final state of the collision products are predicted. The Monte Carlo simulation involves two steps. The first step (the generator) consists in generating events of $p \bar{p}$ collisions at $\sqrt{s}=1.96 \mathrm{TeV}$. Events thus generated contain information about the collision products beginning at the parton level and including all steps of their evolution into particles which will interact with the detector. However, the generator does not simulate interactions with the detector which will determine acceptance and sub-component efficiency. These are part of the second step: the detector simulation. After the detector simulation, the set of variables available for each MC event is the same as that of a real data event. MC samples and data can therefore be analyzed using the same software. The Monte Carlo samples used in this work are summarized in Table 5.1.

\subsubsection{The signal sample}

The mSUGRA phenomenology depends on 5 parameters (see Chapter 1). A scan of this parameter space has been performed to identify the most promising regions to search for the SUSY signal [5][6]. The results of this study are shown in Fig. 5.3 in the $m_{0}-m_{1 / 2}$ plane. The mSUGRA point which maximizes the effective cross section of charginoneutralino production into three leptons was chosen as benchmark. This point is characterized by the following set of parameters:

$$
A_{0}=0, \tan \beta=5, m_{0}=100 \mathrm{GeV} / \mathrm{c}^{2}, \mathrm{~m}_{1 / 2}=180 \mathrm{GeV} / \mathrm{c}^{2}, \mu>0
$$




\begin{tabular}{|l|c|c|c|}
\hline MC Sample & Luminosity in $\mathrm{pb}^{-1}$ & $\sigma_{N L O} \times \mathrm{BR}($ leptons $)$ in $\mathrm{pb}$ & Generator \\
\hline \hline SUSY signal & $\sim 350000$ & 0.642 & Pythia \\
\hline$Z Z$ & $\sim 540000$ & 0.299 & Pythia \\
$W^{ \pm} Z$ & $\sim 430000$ & 0.279 & MadGraph \\
$W^{ \pm} W^{ \pm}$ & $\sim 34000$ & 12.4 & Pythia \\
$Z / \gamma^{*} \rightarrow l l$ & $\sim 4300$ & 23775 & Pythia \\
$t \bar{t}$ & $\sim 170000$ & 6.7 & Pythia \\
$b \bar{b}$ & $\sim 140$ & $6.7 \times 10^{6}$ & Pythia \\
\hline
\end{tabular}

Table 5.1: Summary of the Monte Carlo samples used in the analysis.

which corresponds to $\tilde{\chi}_{1}^{ \pm} \sim 113 \mathrm{GeV} / \mathrm{c}^{2}$.

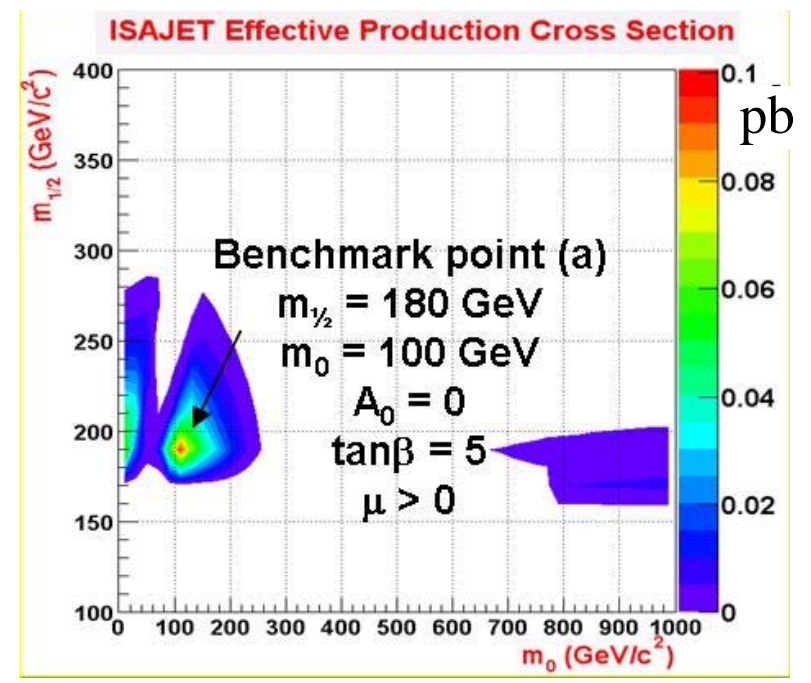

Figure 5.3: Effective production cross section of chargino and neutralino associated production in trilepton final states: $A_{0}=0, \tan \beta=5, \mu>0$.

The signal sample for this benchmark point of the parameter space was produced with the Pythia generator. The sample contains events in which the the chargino and the neutralino are forced to decay leptonically $(l=e, \mu, \tau)$. The branching ratio into three leptons is $22.3 \%$.

\subsubsection{Backgrounds}

Backgrounds events are those events characterized by a similar signature to the signal events, but coming from a different process. The backgrounds relevant to this analysis can be classified as physics backgrounds, coming from a known physics process, or as non-physics backgrounds coming from events with one or two real leptons plus additional contributions from objects which appear to be real leptons. Non-physics backgrounds, otherwise known as "fakes", are estimated from real data and will be decribed in the next section. All the other backgrounds are generated using Monte Carlo simulation (see Table 5.1). Physics backgrounds which have three prompt leptons in the final state include events from $W^{ \pm} Z, Z Z, t \bar{t}, b \bar{b}$ leptonic decay, while backgrounds with two prompt 


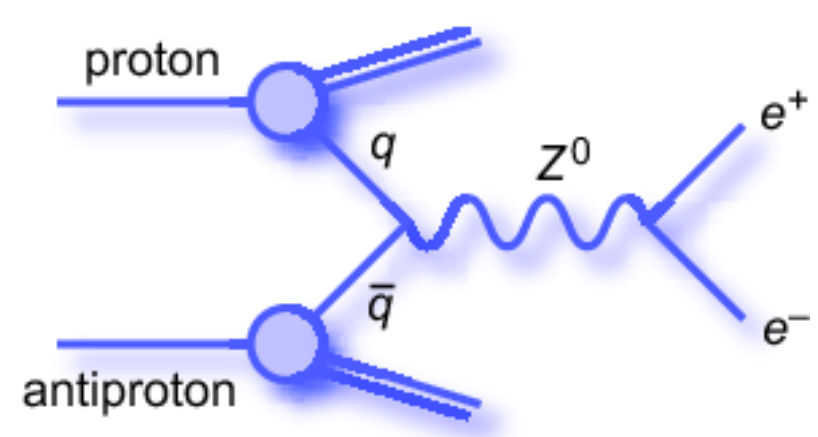

Figure 5.4: Feynman diagram of a Drell-Yan event.

leptons in the final state come from $W^{ \pm} W^{ \pm}, Z / \gamma * \rightarrow l l$ (Drell-Yan) processes. These processes can be subdivided in the following three categories.

$\star$ Drell-Yan: $Z / \gamma * \rightarrow l l$. The Drell-Yan process consists in a quark-antiquark annihilation into a photon or a $Z$ boson which then decay leptonically into a leptonantilepton pair (Fig. 5.4). Yielding two real leptons in the final state, this process is expected to be one of the dominant backgrounds at the stage of dilepton selection. However these events could also lead to three real leptons in the final state if there is an additional radiated photon which converts into a $l^{+} l^{-}$pair. In general higher order processes of $Z / \gamma * \rightarrow l l$ could contribute to the trilepton backgrounds. The Drell-Yan sample used in this analysis is inclusive of all leptonic decays $(l=e, \mu, \tau)$.

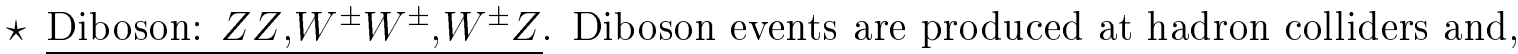
considering the leptonic $Z \rightarrow l l$ and $W \rightarrow l \bar{\nu}$ decays, these events could result in a multilepton signature. More specifically, $Z Z$ and $W^{ \pm} Z$ leptonic decay yields three-real-lepton final states while two real leptons arise from $W^{ \pm} W^{ \pm}$.

$\star \underline{t \bar{t} \text { and } b \bar{b}}$. The electroweak decay of the top quark (Fig. 5.5) produces a bottom quark and an on-shell $W$ boson $(t \rightarrow b W)$, which can subsequently decay into a lepton and a neutrino $W \rightarrow l \nu$, while the bottom quark can decay semileptonically $(b \rightarrow c l \bar{\nu})$. In the $b \rightarrow c l \bar{\nu}$ decay, the $c$ quark takes almost all the momentum, which means that in this case it is difficult to produce high momentum leptons. In the top quark decay the real $W$ takes a huge part of the $t$ quark momentum. Hence the products of the semileptonic $b$ decay are far less energetic than those coming from the $t$ decays. Therefore $t \bar{t}$ events are predicted to contribute more as background in events with high- $p_{T}$ leptons while $b \bar{b}$ events more in the low- $p_{T}$ range. Both $t \bar{t}$ and $b \bar{b}$ leptonic final states can result in three real leptons.

The $t \bar{t}$ sample is generated with a top mass of $178 \mathrm{GeV} / \mathrm{c}^{2}$. The $b \bar{b}$ sample include only direct $b \bar{b}$ production and not gluon splitting nor flavour excitation diagrams (Fig. 5.6). For this reason, a correction factor has been applied to the $b \bar{b}$ cross section to take into account other heavy flavour production mechanisms and the $c \bar{c}$ contribution [7]. 


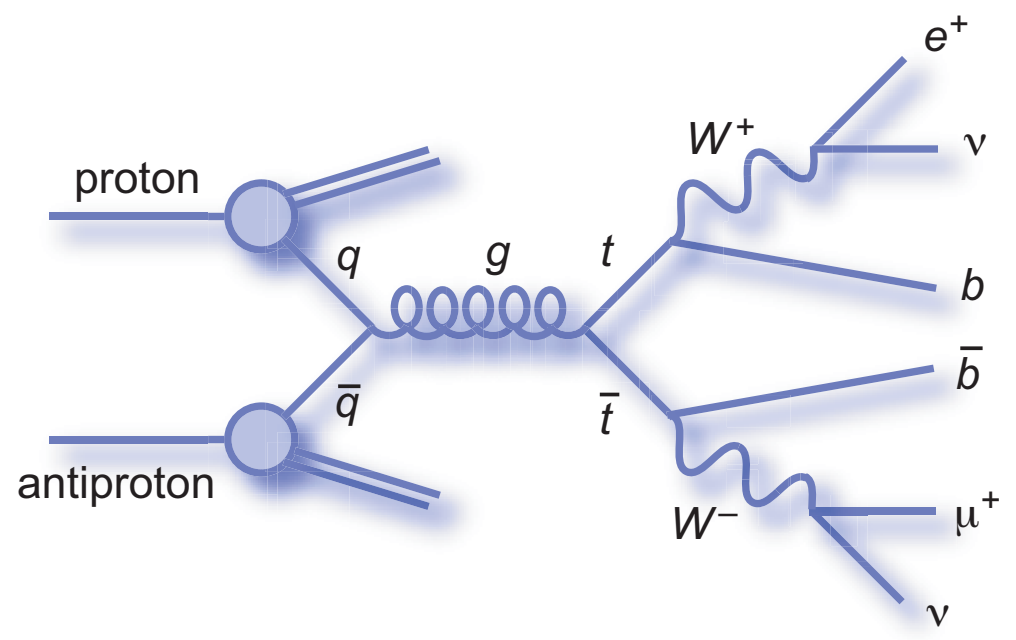

Figure 5.5: Feynman diagram of $t \bar{t}$ production.

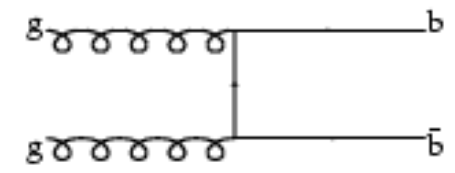

Flavor creation $\left(\alpha^{2}{ }_{\mathrm{a}}\right)$

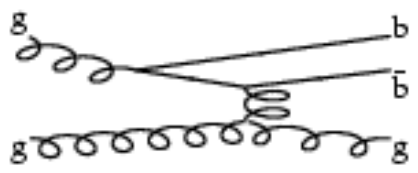

Flavor excitation $\left(\alpha^{3}{ }_{\mathrm{a}}\right)$
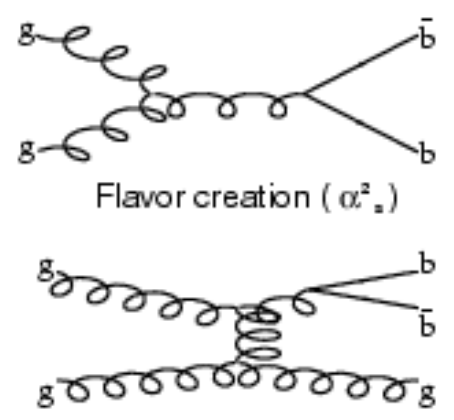

Gluon splitting $\left(\alpha_{\mathrm{s}}^{3}\right)$

Figure 5.6: Feynman diagram of $b \bar{b}$ production. 


\subsection{Fake lepton contribution}

As defined above, the fake leptons are those objects which pass all identification cuts and thus appear as real leptons. The origin of these misidentified leptons are several such as: jets which deposit substantial energy in the EM calorimeter and have a stiff track associated with them that can be identified as an electron; hadronic jets with late showers which leak out of the hadron calorimeter into the muon chambers where they can be identified as muons; leptons non originating in the hard scattering such as decay in flight of kaons and pions to muons.

The fake contribution is estimated in two steps: first, the probability that an object fakes a lepton is calculated; then the results are applied to the data sample used for the analysis.

$\star$ The determination of fake rates. In order to measure the misidentification rates, jet data samples, which have relatively few prompt leptons, have been used. For each lepton type the following ratio is defined:

$$
\frac{\text { fakeable objects which pass the lepton identification cuts }}{\text { fakeable objects }}
$$

where a fakeable object is an object that can produce a fake lepton and electron (muon) fakeable objects are jets (tracks). These ratios are calculated as a function of $E_{T}\left(p_{T}\right)$ of the jet (track). The fake rates used in this thesis have been calculated in $[8]$.

* Applying the fake rates. Several fake contributions have to be accounted for:

$\diamond$ events with one real and one fake lepton (real + fake);

$\diamond$ events with two real and one fake leptons (2 real + fake);

$\diamond$ events with one real and two fake leptons;

$\diamond$ events with three fake leptons.

Since electron fake rates are of the order of $10^{-3}$ and muon fake rates $10^{-2}$, the contributions where there are more than one fake lepton are assumed to be negligible. Therefore only the first two contributions are considered.

The real + fake contribution is calculated as follows. First events with an identified lepton are selected and in each of them fakeable objects are searched for. If a fakeable object is found, it is treated as a real lepton and the event is then passed through the whole analysis code with a weight corresponding to the fake rate of the fake lepton. If a second fakeable object is found in the same data event it is treated as a second fake event. The 2 real + fake contribution is estimated in a similar way after having identified two leptons.

The above is the general procedure to calculate fakes. However, for this particular analysis it is necessery to take into account another effect. If the real + fake contribution were extracted from the dilepton data set it would suffer from a trigger bias because a dilepton trigger requires two triggered leptons. This increases the probability of finding a second real lepton in the event hence influencing the chance of finding fakeable objects. But the fake rates [8] were calculated independently from any trigger. This issue affects only 
the real + fake contribution since the 2 real + fake contribution is estimated by a priori requiring two real leptons consistently with the dilepton trigger. For this reason, the real + fake contribution is estimated from a single lepton trigger sample, while the 2 real + fake from the data sample used in the analysis. 



\section{Bibliography}

[1] J. Heinrich, Benefits of Blind Analysis Techniques, CDF internal note 6576, 2003.

[2] M. Spiropulu, A Blind Search for Supersymmetry using the Missing Energy plus Multijet channel, CDF internal note 5448, 2000.

[3] The Trigger and Datasets Working Group, CDF Run-II Trigger Table and Datasets Plan, CDF internal note 4718, Dec 2001.

[4] M.P. Giordani, S. Lammel, Dilepton Triggers for Exotic Searches at CDF II, CDF internal note 6074, Aug 2002.

[5] D. Bortoletto et Al., SugraScan package implementation and validation, CDF internal note 6968, Jul 2004.

[6] M.P. Giordani et Al., SugraScan: Investigating the Phenomenology of Beyond the Standard Model Scenarios, CDF internal note 6128, Sep 2002.

[7] S. Dube et Al., Searches for Chargino-Neutralino Production in mSUGRA Model in a Di-electron+track Channel, CDF internal note 7478, 2005.

[8] M. Griffiths et Al., Fake Rate For Low- $p_{T}$ Leptons, CDF internal note 7470, May 2005. 



\section{Chapter 6}

\section{The Data analysis}

Having described the analysis tools in the previous chapters, the core of the analysis is presented in the following. In the first section of this chapter the procedure for a comparison of data and Monte Carlo is described. In the second part, the event selection is presented.

Before analyzing events with one electron and one muon, events with two muons and two electrons are considered in order to test our procedure with a high statistics samples and gain confidence in the lepton selection. Dimuon and dielectron events may be considered as additional control regions. The main analysis cuts are then justified in section 6.4. Following the blind analysis approach, in the next section the control regions are studied. The last section is dedicated to the description of the current status of the $e \mu$ analysis.

\subsection{Data and MC comparison}

When comparing data and MC it is necessary to normalize the MC integrated luminosity to the luminosity used to generate the data set. Moreover because some details of the detector are not completely simulated, some final corrections need to be applied to the Monte Carlo events to account for these deficiencies. More specifically, these corrections regard the trigger and identification efficiencies.

* Correction for the trigger efficiency. The CDF data-taking structure is such that a number of lower level triggers select and pass data to higher level triggers. The leptons in an event generally activate more than one trigger. As a consequence, a data set contains events which could have been recorded via more than one trigger path. When comparing data with Monte Carlo events it is necessary to take the quality of the trigger response in collecting data into account. In order to correct the effect of inefficiencies due to the technical limitations of the detector, a weight corresponding to the total efficiency of the trigger must be applied to MC events. The leptonic trigger paths responsible for collecting the collision data have efficiencies which often depend on the momentum of the lepton. The efficiencies are measured as outlined in Appendix B and the discrete numbers of measurements are parametrized by turn-on curves which are functions of the lepton $p_{T}$. Efficiencies of different trigger levels are measured separately and are multiplied together to obtain the total efficiency: this is the probability for an event to activate the trigger 
of interest.

The trigger efficiency corrections are applied on an event-by-event basis to the MC events. Each lepton of a MC event is examined. The statistical weight of each $\mathrm{MC}$ event is then multiplied by the trigger efficiency yielding a new weight for the event. Several measurements of the SUSY DILEPTON trigger efficiencies have been performed. In this thesis the efficiencies obtained in [1][2] were used, together with the measurements described in Appendix B.

* Correction for the identification efficiency. Lepton identification efficiency reflects the fraction of positively identified leptons which is generally different for real data and $\mathrm{MC}$ events. This is a consequence of the non-ideal performance of the detector simulation which tends to overestimate the identification efficiencies due to not having included some subtle detector effects. These are small effects and are therefore left to be handled by efficiency corrections. For each lepton type an identification efficiency is calculated both for MC and data events. The relative efficiencies (scale factors) takes into account the different effect of identification cuts on MC and data and were calculated in [3][4][5][6][7]. The numbers used in this analysis are listed in Tab.6.1. The weight of the MC events is then corrected by the product of the efficiencies of the identified leptons in the event.

\begin{tabular}{|l|c|}
\hline Lepton type & Scale factors \\
\hline \hline Central electron with $4 \leq E_{T}<8 \mathrm{GeV}$ & $1.00 \pm 0.2$ \\
Central electron with $8 \leq E_{T}<20 \mathrm{GeV}$ & $1.03 \pm 0.015$ \\
Central electron with $E_{T} \geq 20 \mathrm{GeV}$ & $0.996 \pm 0.005$ \\
Central CMUP muon with $4 \leq p_{T}<20 \mathrm{GeV} / \mathrm{p}$ & $0.85 \pm 0.05$ \\
Central CMUP muon with $p_{T} \geq 20 \mathrm{GeV} / \mathrm{p}$ & $0.881 \pm 0.010$ \\
\hline
\end{tabular}

Table 6.1: Relative identification efficiencies applied to each identified lepton in $M C$ events.

\subsection{Event selection}

The number of events in the SUSY DILEPTON data set is around 30 million. A preliminary filter is applied to data events in order to reduce the total number of events which will be subjected to full data analysis.

Events are kept if two central leptons $(|\eta|<1.1)$ are identified using the identification requirements described in Chapter 4.4 and if their transverse momentum is greater than 8 and $5 \mathrm{GeV} / \mathrm{c}$ for the leading and the next-to-leading leptons respectively. Applying this filter and the "good run list" the number of events is reduced by a factor of 10 .

Before proceeding with the analysis, conversion candidates and cosmics are removed by means of the standard CDF removal algorithms (see 4.4). Events have also to fulfill the following requirements:

\footnotetext{
${ }^{1} \mathrm{~A}$ good run depends on the condition of sub-detectors as described in 5.3.1. The good run list used in this analysis is referred as Good run list v7 no silicon. See web page [3] for more details.
} 


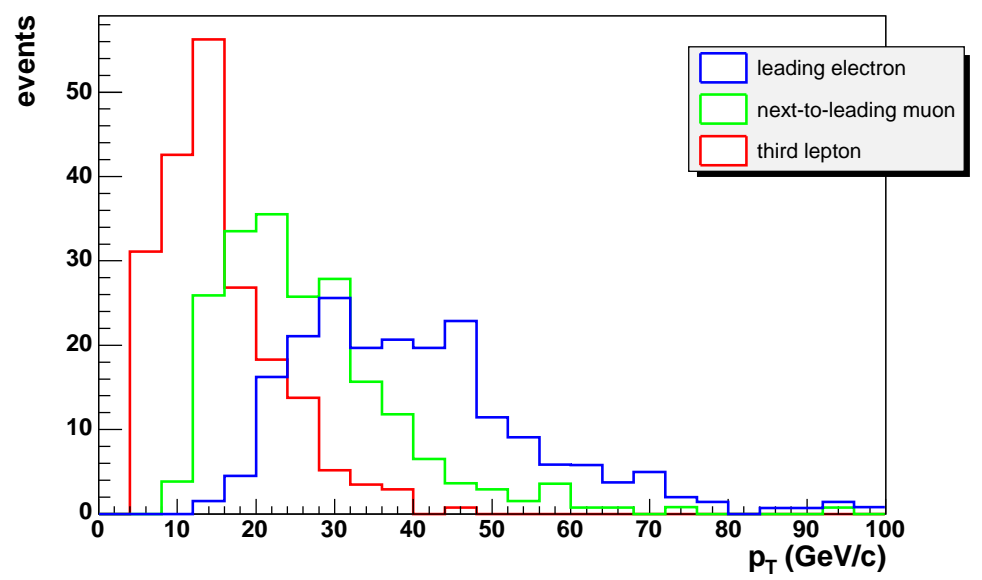

Figure 6.1: Transverse momentum distribution for $M C$ events in which three leptons coming from the associated chargino-neutralino production are reconstructed.

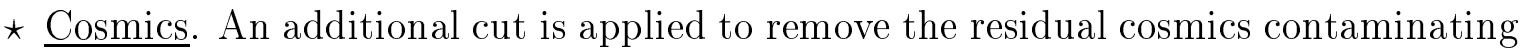
the sample: it is an explicit geometrical requirement on the opening angle between two identified muons in the event. Because cosmic muons are expected to present a back to back topology, the event is rejected if this angle is greater than $175^{\circ}$.

* Lepton momentum thresholds. Given the momentum threshold at trigger level $(4 \mathrm{GeV})$ and the expected $p_{T}$ spectrum of leptons arising from chargino and neutralino production (Fig. 6.1) the leading and next-to-leading leptons are required to have $p_{T, 1}>10 \mathrm{GeV} / \mathrm{c}$ and $p_{T, 2}>5 \mathrm{GeV} / \mathrm{c}$ respectively.

The first two lepton types are selected in accordance with the trigger under study. When considering dielectron events, for which the DIELECTRON_CENTRAL4 trigger is used, two CEM electrons are required. Similarly, for dimuon events, which are collected with the CMUPCMUP4 trigger, events are required to must have two central CMUP muons passing all offline identification cuts. For the selection of $e \mu$ events, which are collected by CEM4_CMUP4 trigger, events with one central CEM electron and one central CMUP muon are selected.

After selecting the first two leptons, the third lepton is selected independently of the trigger from a class which includes CEM electrons and CMUP or CMX muons with $p_{T}>4 \mathrm{GeV} / \mathrm{c}$, in order to maximize the acceptance for leptons at low momentum.

* Vertex requirements. At least one vertex of quality 12 (see Tab. 4.2) is asked in each event. Among the identification cuts is the requirement that tracks associated with leptons must have $z_{\text {vertex }} \leq 60 \mathrm{~cm}$ assuring their origin in the interaction region. In addition tracks are required to originate from an interaction vertex Therefore a cut on the $|\Delta z|<4 \mathrm{~cm}$ between each lepton and the vertex is applied. To guarantee that all leptons come from the same vertex it is also required $|\Delta z|<4 \mathrm{~cm}$ between each lepton pair.

$\star$ Jet selection. Jets are reconstructed with a cone algorithm as described in Chapter 4.3 and the jet corrections described are applied. Moreover a jet is considered in the analysis only if: 
$\diamond\left|\eta_{j e t}\right|<2.5$

$\diamond E_{T}>5 \mathrm{GeV}$ before any correction;

$\diamond E_{T}>20 \mathrm{GeV}$ after all corrections;

$\diamond$ electromagnetic fraction of the energy $<0.9$.

$\star$ Missing energy. The missing transverse energy is corrected for the corrections applied to jets and the presence of identified muons in the event according to the procedure described in Chapter 4.5.

$\star$ Lepton isolation. Because all possible decays which yield three leptons from the chargino-neutralino production do not contain jets, there should be little energy around each lepton. So that, leptons are expected to be well isolated.

Isolation cuts are therefore applied at the identification stage. In addition, leptons are required to be well separated from each other: $\Delta R=\sqrt{\Delta \eta^{2}+\Delta \phi^{2}}>0.4$. The same separation is also required between each lepton and each reconstructed jet. These isolation requirements remove part of the heavy flavour contributions where leptons are generally folded in jets.

$\star$ Invariant mass cut. In order to limit the size of the generated dataset, Monte Carlo samples generally have specific requirements at generator level which limit the lepton transverse momentum and/or the invariant mass of lepton pairs from the hard scattering. Such requirements are applied in some of the Monte Carlo samples used in this analysis, such as the Drell-Yan which have a cut on the invariant mass of two leptons at $5 \mathrm{GeV} / \mathrm{c}^{2}$. For this reason the low invariant mass region is not expected to be well reproduced by the MC events. Therefore the minimum invariant mass of the first two leptons is required to be $\geq 15 \mathrm{GeV} / \mathrm{c}^{2}$.

Moreover, when selecting three leptons which can be electrons or muons, opposite charge leptons of the same flavour are required to have invariant mass $M_{l^{+} l^{-}}<$ $76 \mathrm{GeV} / \mathrm{c}^{2}$ and $M_{l^{+} l^{-}}>106 \mathrm{GeV} / \mathrm{c}^{2}$ to remove the $Z$ boson peak. The invariant mass of any lepton pair is also required to be above $15 \mathrm{GeV} / \mathrm{c}^{2}$.

\subsection{The dielectron and dimuon events}

Before moving to the $e \mu$ channel, dielectron and dimuon events are considered. The idea is to verify leptonic identification on samples with high statistics where a classic "standard candle" is at disposal: the $\mathrm{Z}$ peak.

The whole invariant mass spectrum is shown in Fig. 6.2 and Fig. 6.4: the invariant mass, the missing energy, the number of jets, the leading lepton $p_{T}, \Delta R$ and $\Delta \phi$ of the two leading leptons are shown. The same distributions are presented in Fig. 6.3 and Fig. 6.5 for the $\mathrm{Z}$ mass window only, defined as the $76-106 \mathrm{GeV} / \mathrm{c}^{2}$ mass range. All plots show data compared to $\mathrm{MC}$ whose different background contributions are stacked one on the top of the other. In all the distributions agreement is within data statistical uncertainties.

Finally, the number of expected and observed $Z$ events are compared: in dimuon events $810 Z$ events are observed in data versus $828.5 \pm 1.5$ expected in Drell-Yan Monte Carlo; in dielectron events 3354 and $3320 \pm 3$ events are observed and expected respectively. 
Considering that quoted errors are only due to the statistics ${ }^{2}$ of the $\mathrm{MC}$, these numbers are deemed to be in reasonable agreement.

\subsection{The $e \mu$ channel: effect of the analysis cuts}

The analysis signature under study is characterized by three leptons and some missing energy. In particular, this thesis focuses on events with one electron, one muon and one additional lepton. The sequential cuts which select the signature are:

1. selection of events with 2 leptons, one electron and one muon, with invariant mass greater than $15 \mathrm{GeV} / \mathrm{c}^{2}$;

2. the missing transverse energy must be greater than $15 \mathrm{GeV}$ for the presence of LPSs and a neutrino in the final state;

3. events must have a third lepton. The invariant mass for each lepton pair must be greater than $15 \mathrm{GeV} / \mathrm{c}^{2}$ and must be outside the $\mathrm{Z}$ window when the lepton pair involves two same flavor leptons with opposite charge.

The effect of the three sequential cuts for for events with one electron and one muon have been studied comparing signal and background MC events. It is summarized in Tab. 6.2 and corresponding distributions of invariant mass and missing energy are shown in Fig. 6.6-6.7-6.8 where shown MC backgrounds overlap the signal distribution. The missing transverse energy signal distribution in 6.6 provides justification for neglecting the range $\mathbb{E}_{T}<15 \mathrm{GeV}$.

Sequentially applying cuts 1,2 and 3 leads to a substantial reduction of the SM model background. After applying the first requirement of two leptons, the main background contributions comes from Drell-Yan and heavy flavor processes. The missing energy cut completely removes the $b \bar{b}$ contribution and reduces the Drell-Yan events by a factor of three. The third lepton requirement kills almost all the remaining SM processes and the main backgrounds left are $W^{ \pm} Z$ and some Drell-Yan.

As a figure of merit the ratio $S / \sqrt{B}$ has been considered: after cut $1 S / \sqrt{B}=0.079$, while after cut $3 S / \sqrt{B}=0.422$. This leads to conclude that the three sequential cuts described above provide a significant enhancement in $S / \sqrt{B}$, but signal and SM backgrounds are still of the same order of magnitude.

\footnotetext{
${ }^{2}$ Based on other searches for the trilepton signature of the chargino-neutralino production at $\mathrm{CDF}$ $[9][10][11][12]$, systematic uncertainties are $\sim 10 \%$. Because of time constraints systematic errors for MC have not yet been estimated. MC errors quoted in this chapter are only statistical.
} 

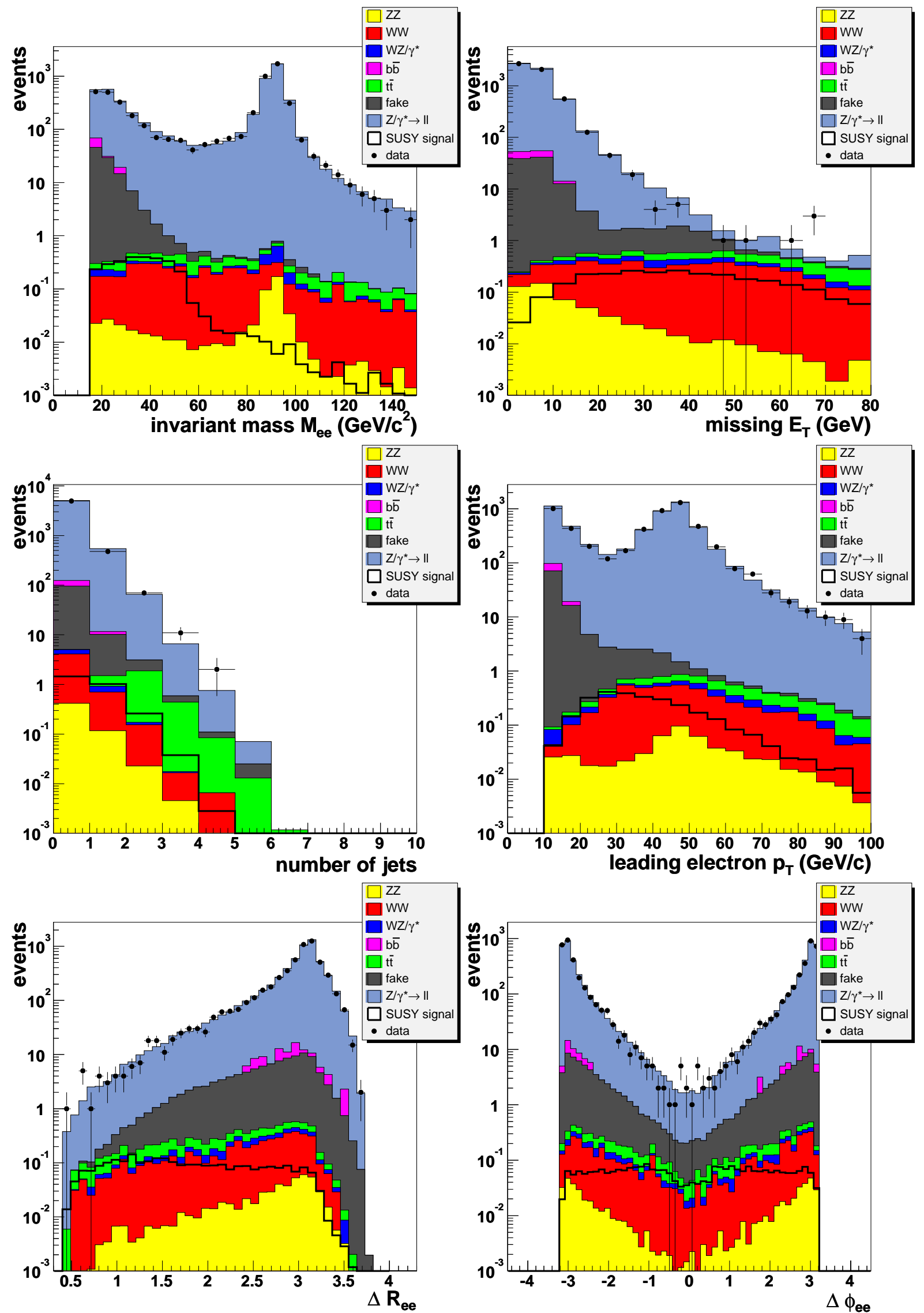

Figure 6.2: Opposite sign dielectron distributions: the whole invariant mass spectrum. 

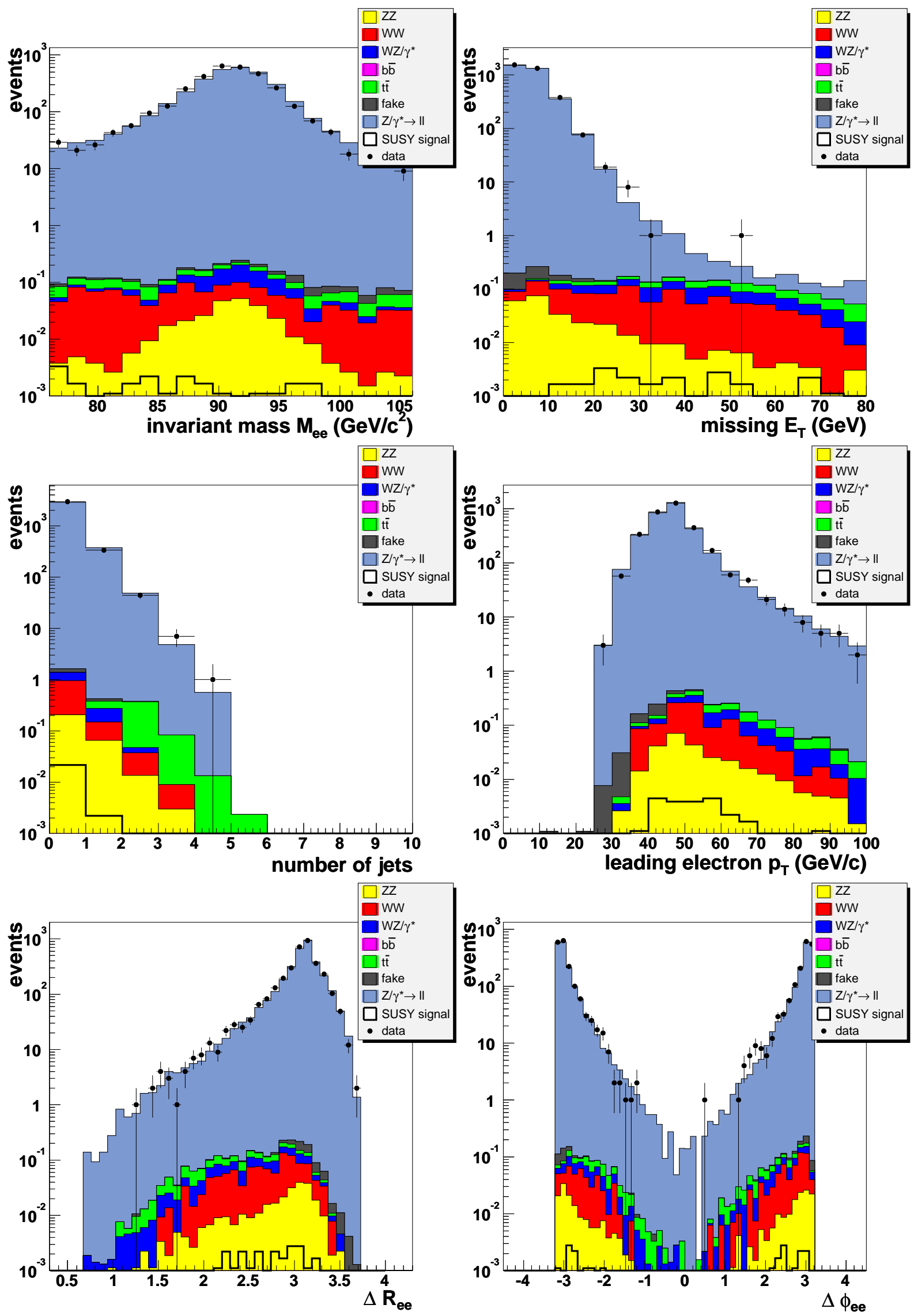

Figure 6.3: Opposite sign dielectron distributions: the $Z$ mass window. 

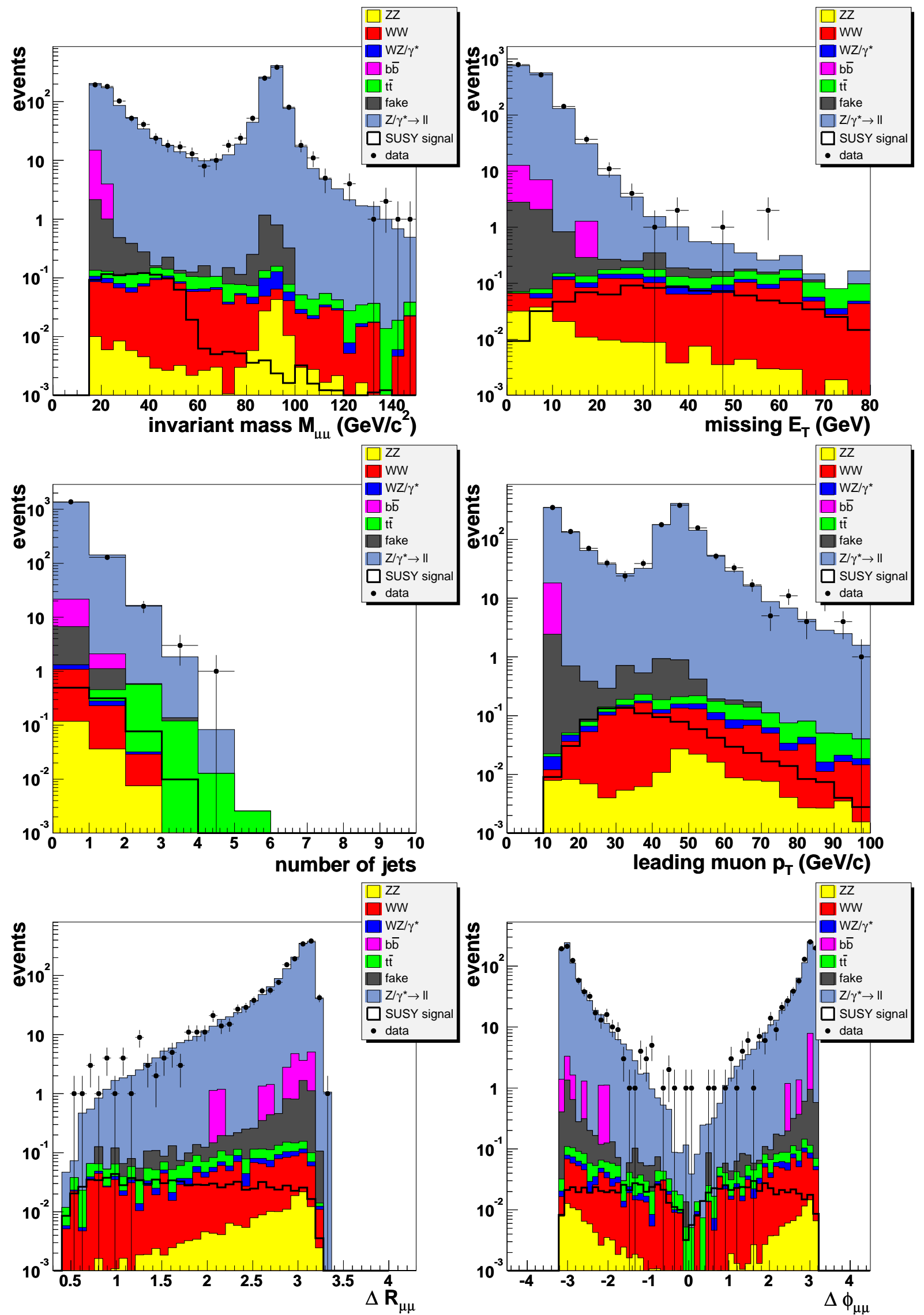

Figure 6.4: Opposite sign dimuon distributions: the whole invariant mass spectrum. 

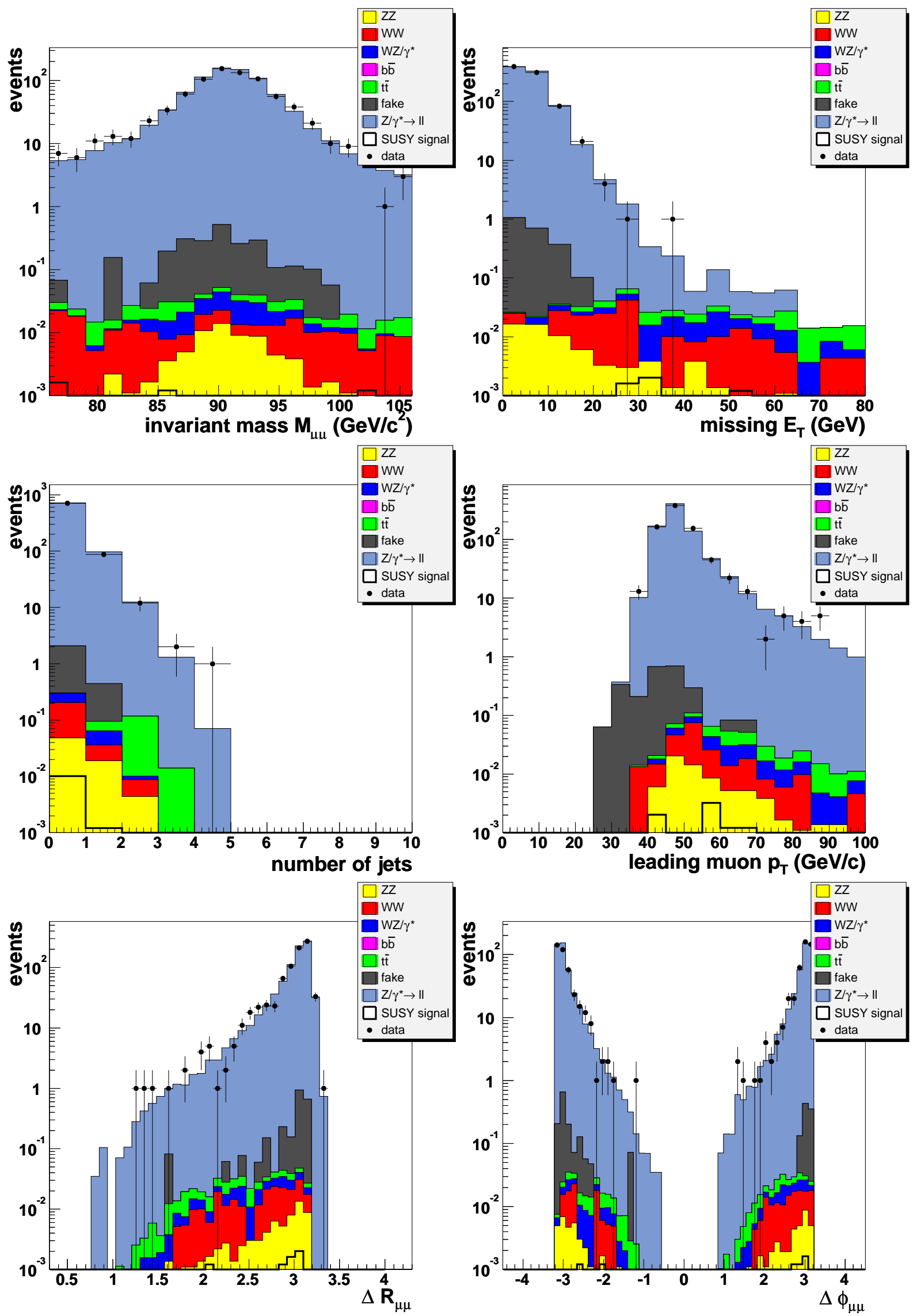

Figure 6.5: Opposite sign dimuon distributions: the $Z$ mass window. 


\begin{tabular}{|l|c|c|c|}
\hline & expected events after cut 1 & expected events after cut 2 & expected events after cut 3 \\
\hline \hline$Z Z$ & $0.254787 \pm 0.000212$ & $0.156688 \pm 0.000166$ & $0.021028 \pm 0.000061$ \\
$W^{ \pm} Z$ & $0.653284 \pm 0.000422$ & $0.595318 \pm 0.000403$ & $0.057251 \pm 0.000125$ \\
$W^{ \pm} W^{ \pm}$ & $4.302090 \pm 0.013749$ & $3.796012 \pm 0.012915$ & $0.000000 \pm 0.000000$ \\
$Z / \gamma^{*} \rightarrow l l$ & $59.584950 \pm 0.403115$ & $21.176546 \pm 0.240319$ & $0.038613 \pm 0.010262$ \\
$t \bar{t}$ & $3.005177 \pm 0.002266$ & $2.881635 \pm 0.002219$ & $0.001001 \pm 0.000041$ \\
$b \bar{b}$ & $50.858913 \pm 11.534680$ & $0.000000 \pm 0.000000$ & $0.000000 \pm 0.000000$ \\
\hline Total BG & $118.659201 \pm 11.541730$ & $28.606199 \pm 0.240676$ & $0.117893 \pm 0.010263$ \\
Signal & $0.863441 \pm 0.000574$ & $0.763378 \pm 0.000539$ & $0.142323 \pm 0.000233$ \\
\hline$S / \sqrt{B}$ & 0.079 & 0.141 & 0.422 \\
\hline
\end{tabular}

Table 6.2: Effect of the analysis cuts applied sequentially on background and signal $M C$ when selecting $\mathrm{e} \mu$ events.

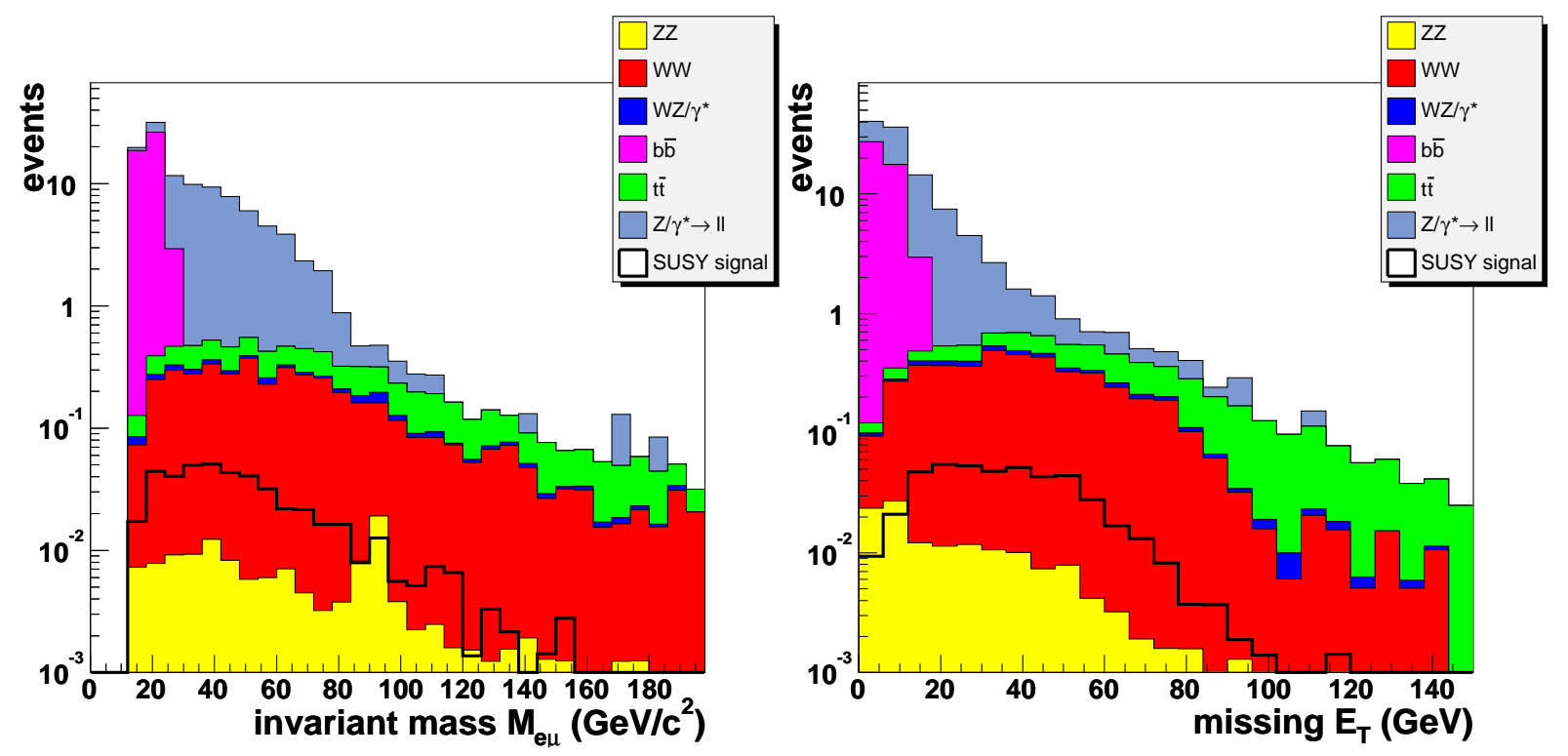

Figure 6.6: Opposite sign e $\mu$ distributions for $M C$ events after cut 1: the invariant mass and the missing energy spectra. 


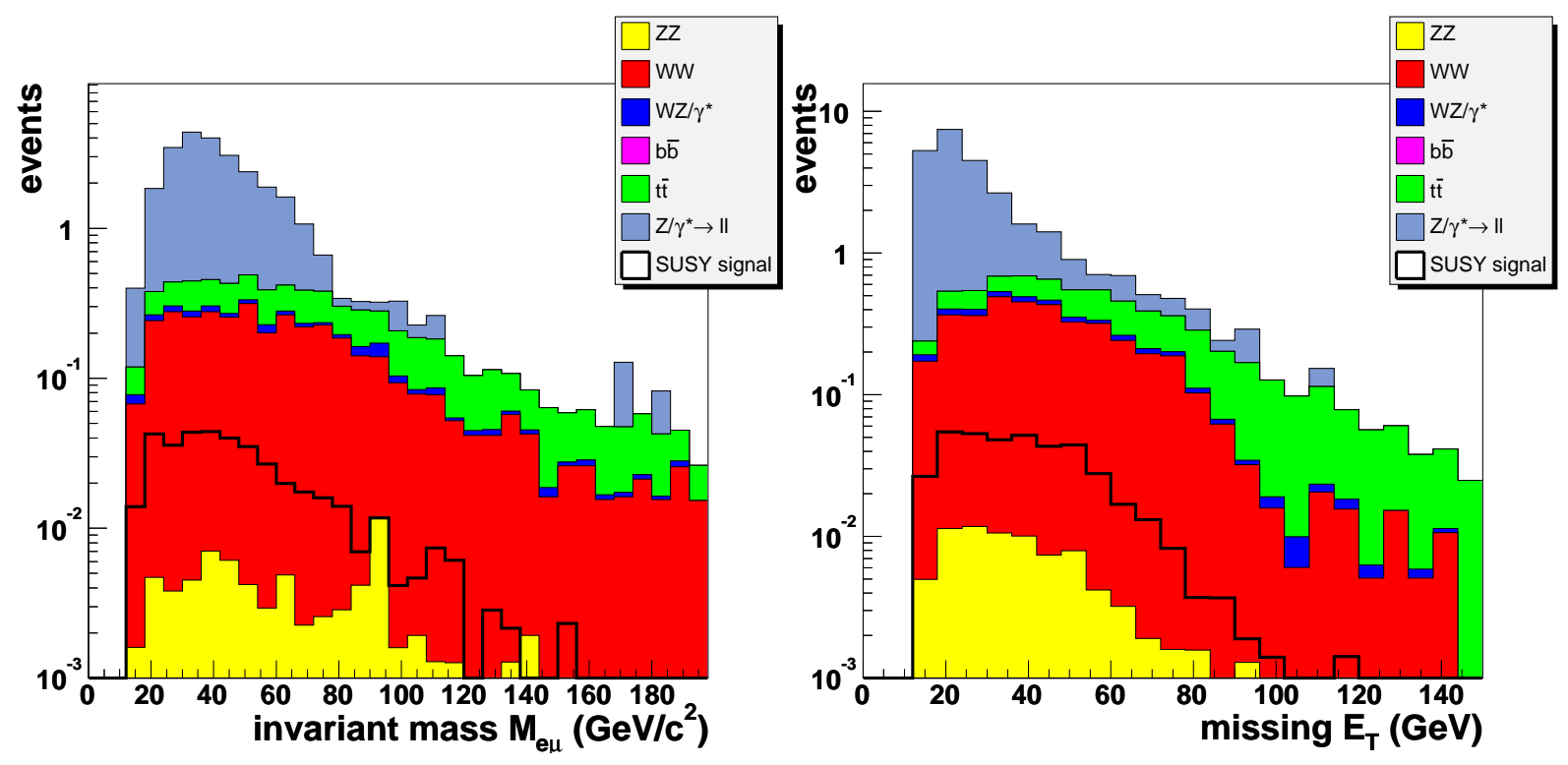

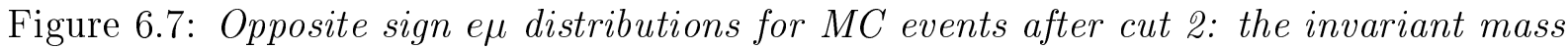
and the missing energy spectra.

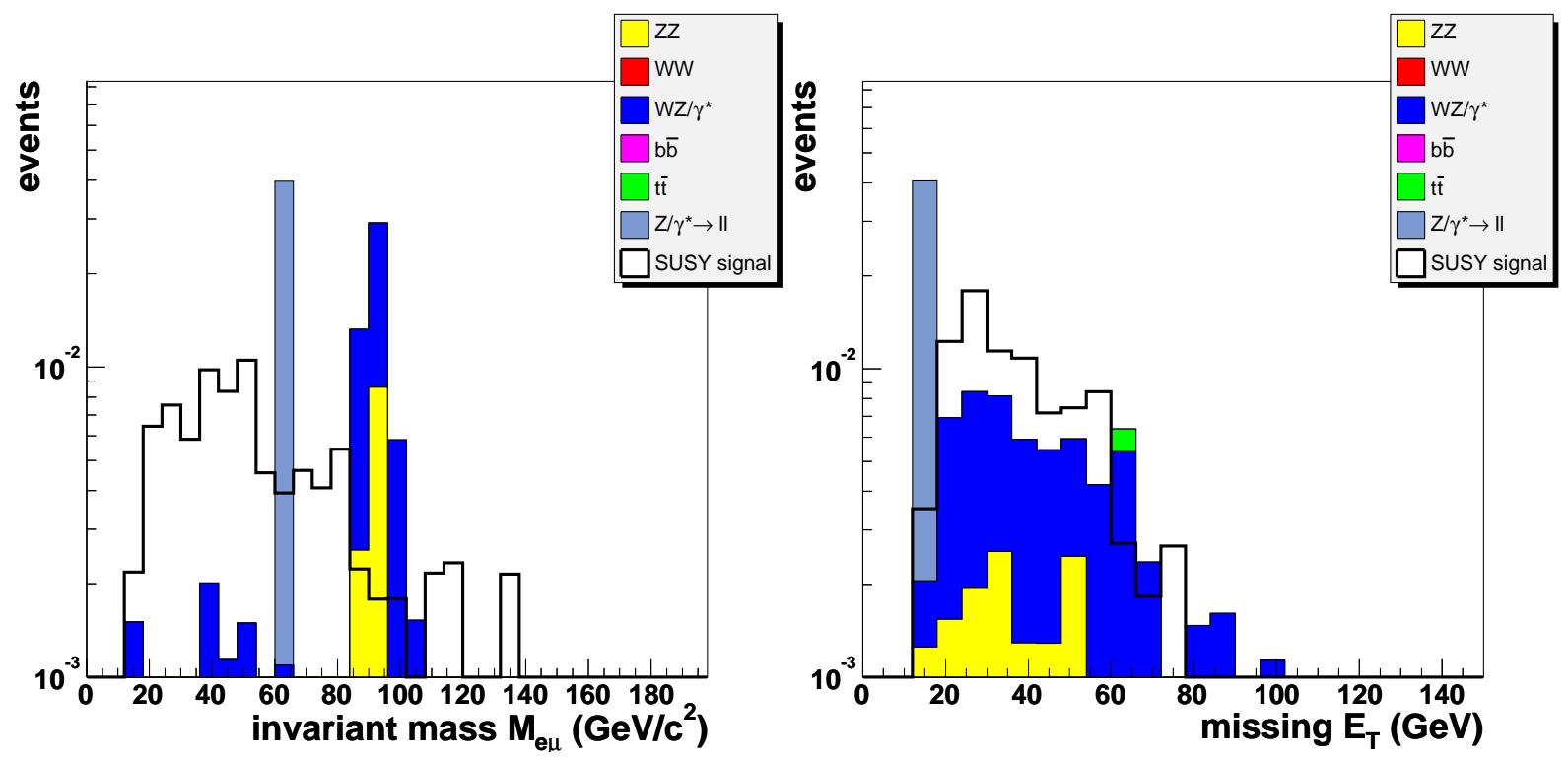

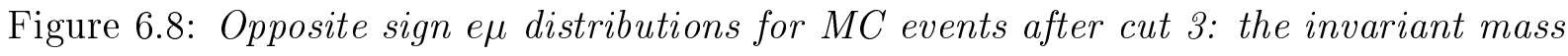
and the missing energy spectra. 


\subsection{Control regions for $e \mu$ events}

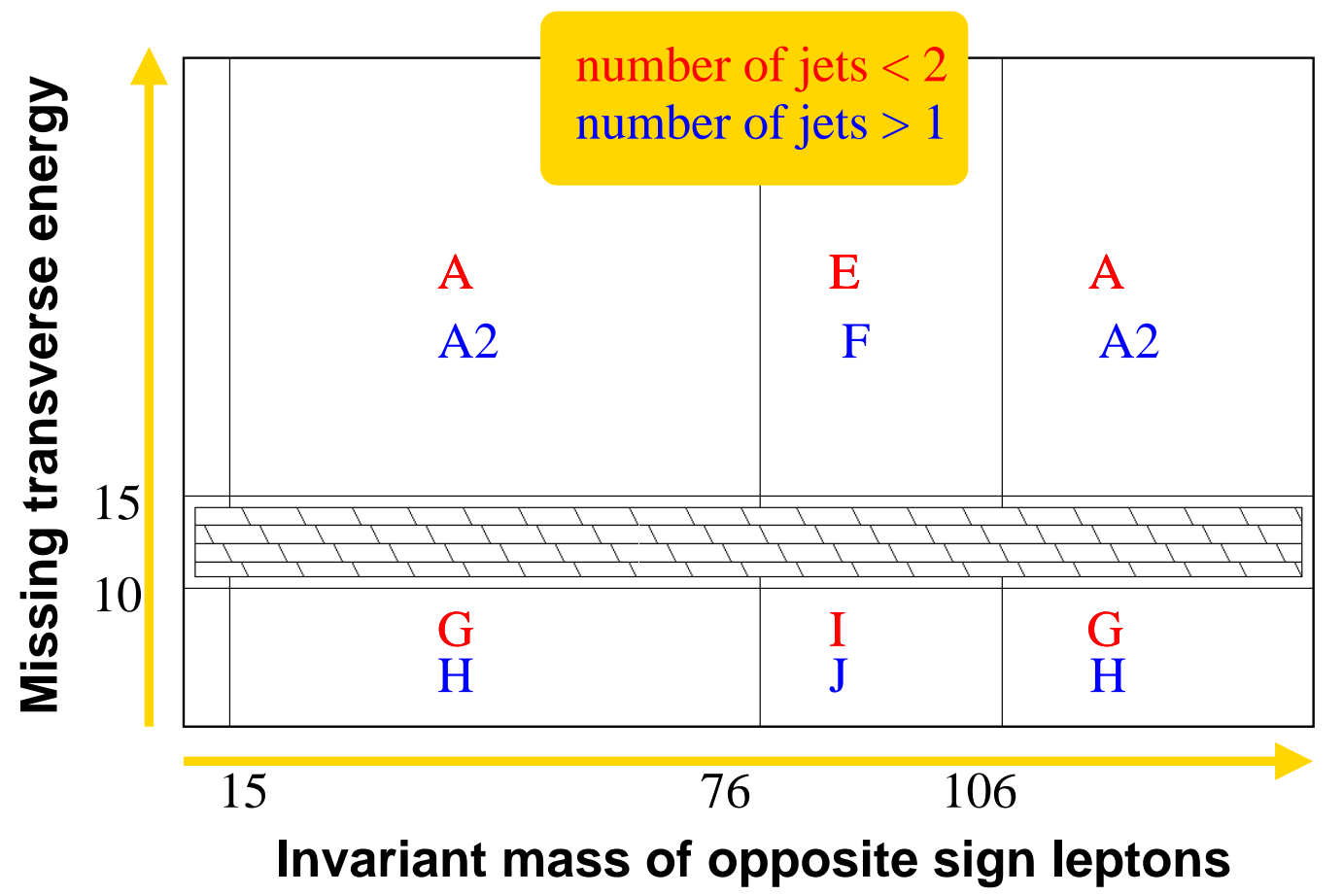

Figure 6.9: Grafical representation of control regions.

As introduced in Chapter 5.2 the analysis is performed as a blind analysis: the signal region is not investigated until the capability to predict the SM backgrounds in control regions is demonstrated. Fig. 6.9 shows a graphical representation of the control regions considered for other trilepton channels of the chargino-neutralino analysis at CDF. Both high and low jet activity scenarios are considered. Each letter in the $x-y$ plane, where $y$ is the $\mathbb{E}_{T}$ and $x$ is the invariant mass, correspond to a specific range of missing energy and invariant mass of the two leading leptons. High and low missing transverse energy range are separated by a gap of $5 \mathrm{GeV}$ in order to be independent on the exact position of the high-low range threshold. On the x-axis the invariant mass of the two leading leptons is subdivided into zones inside and outside the $Z$ mass window. However no $Z$ peak is expected in the $e \mu$ selection therefore the control regions for this specific channel have been taken to be:

$\star \mathbf{A}+\mathbf{E}$ region with high $\mathbb{E}_{T}$ and low jet activity;

$\star \mathbf{G}+\mathbf{I}$ region with low $\mathbb{E}_{T}$ and low jet activity;

$\star \mathbf{A} 2+\mathbf{F}$ region with low $\mathbb{F}_{T}$ and high jet activity;

$\star \mathbf{H}+\mathbf{J}$ region with low $\mathbb{E}_{T}$ and high jet activity.

These control regions are considered both at the stage of the dilepton and trilepton selection. The regions at high $\mathbb{E}_{T}$ are important in understanding the missing energy resolution. The low $\mathbb{E}_{T}$ region is expected to be dominated by Drell-Yan processes which have 
no source of missing energy. The high jet multiplicity region is expected to be sensitive to the heavy flavour contributions. Same sign (SS) and opposite sign (OS) charge leptons are considered separately because the main contribution to same sign events are expected to be fake leptons, given that almost no SM processes lead to two leptons of the same sign in the final state. It follows that separating SS and OS events helps in verifying the fake lepton modelling procedure.

One can also distinguish between $e \mu$ and $\mu e$ channels by specifying:

$\diamond e \mu$ channel when $p_{T, e}>10 \mathrm{GeV} / \mathrm{c}$ and $p_{T, \mu}>5 \mathrm{GeV} / \mathrm{c}$;

$\diamond \mu e$ channel when $p_{T, m u}>10 \mathrm{GeV} / \mathrm{c}$ and $p_{T, e}>5 \mathrm{GeV} / \mathrm{c}$.

These two channels are not exclusive in the high momentum range where, for instance, an event with $p_{T, e}=20 \mathrm{GeV} / \mathrm{c}$ and $p_{T, \mu}=20 \mathrm{GeV} / \mathrm{c}$ falls in both categories. They are well separated in the low momentum range where they provide another handle for verifying $b \bar{b}$ production and the modelling of fakes at low momentum where they are expected to be the dominant backgrounds.

Before looking at control regions, distributions of the whole mass spectra for $e \mu$ and $\mu e$ events are shown in Fig. 6.10-6.11 and in 6.12-6.13 respectively. In OS events data distributions follow MC shapes except for the low missing energy region. In SS events data and MC shapes are similar but number of events in data are generally larger that in the background.

\subsubsection{Dilepton control regions}

In Tables 6.3-6.4 the summary of number of events in dilepton control regions are presented. Distributions of $e \mu$ and $\mu e$ events are presented for each control region in Fig. 6.146.16-6.18-6.20 and in 6.15-6.17-6.19-6.21 respectively. Consistently with the whole invariant mass distributions presented in the previous section distributions in control regions present a general good agreement except for the $\mathbf{G}+\mathbf{I}$ region which is the low missing energy region with low jet multiplicity. Apart the $\mathbf{G}+\mathbf{I}$, other SS control regions do not present particular discrepancies between data and MC at level of statistical accurancy (only few events). 

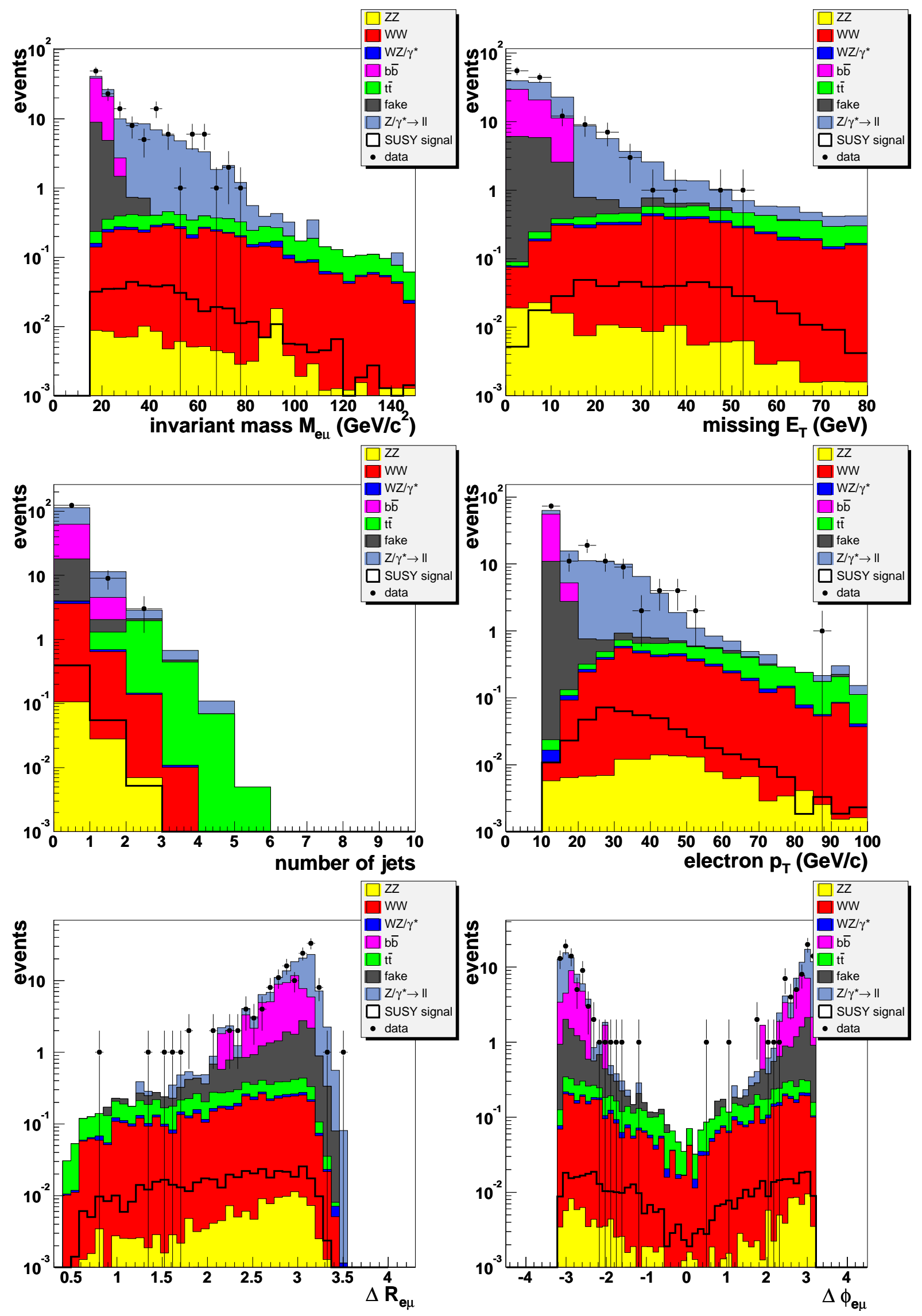

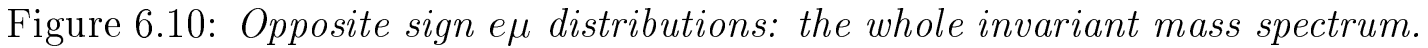



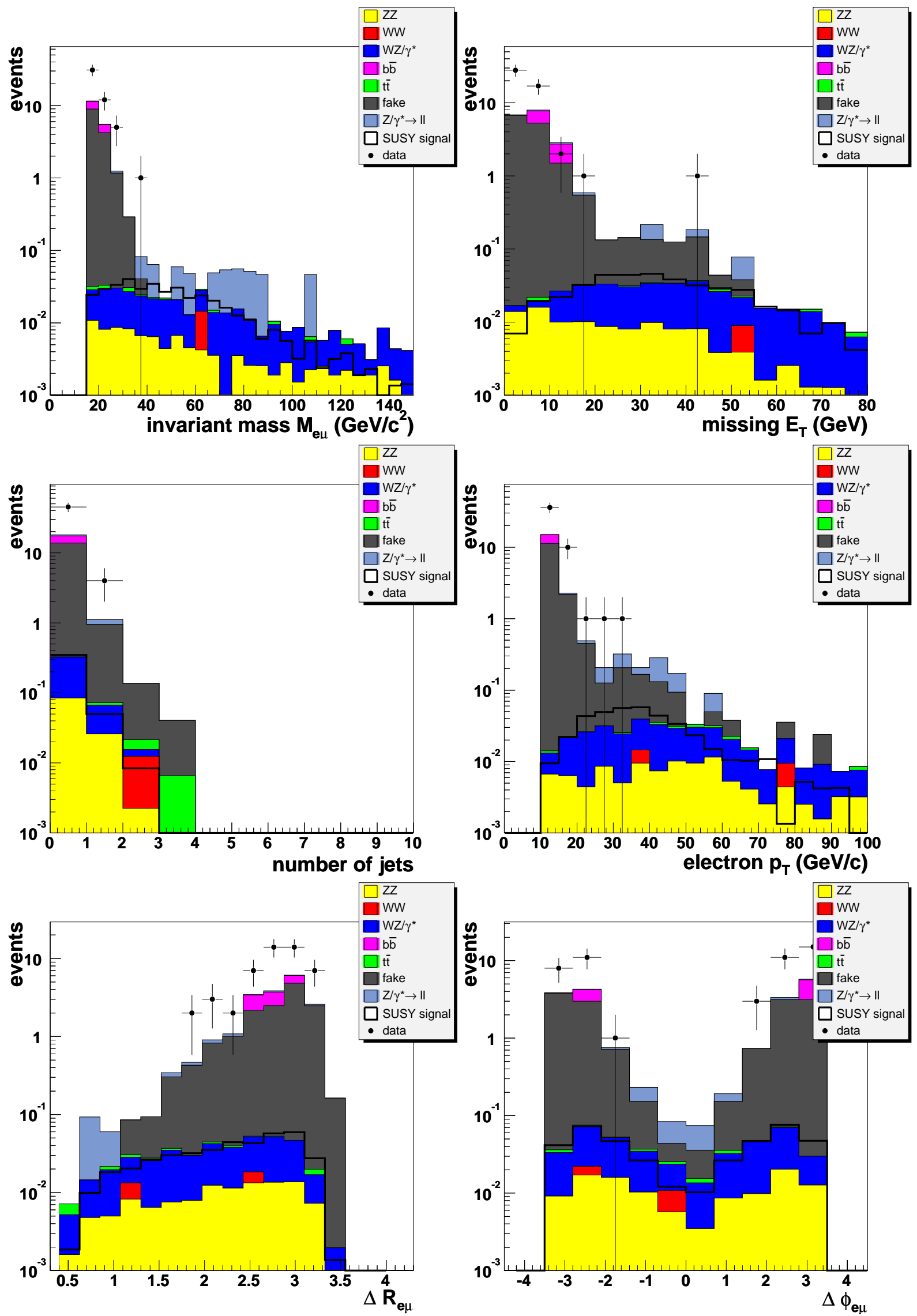

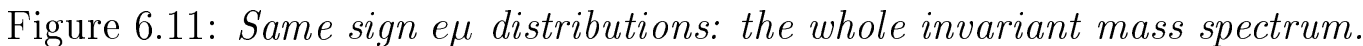



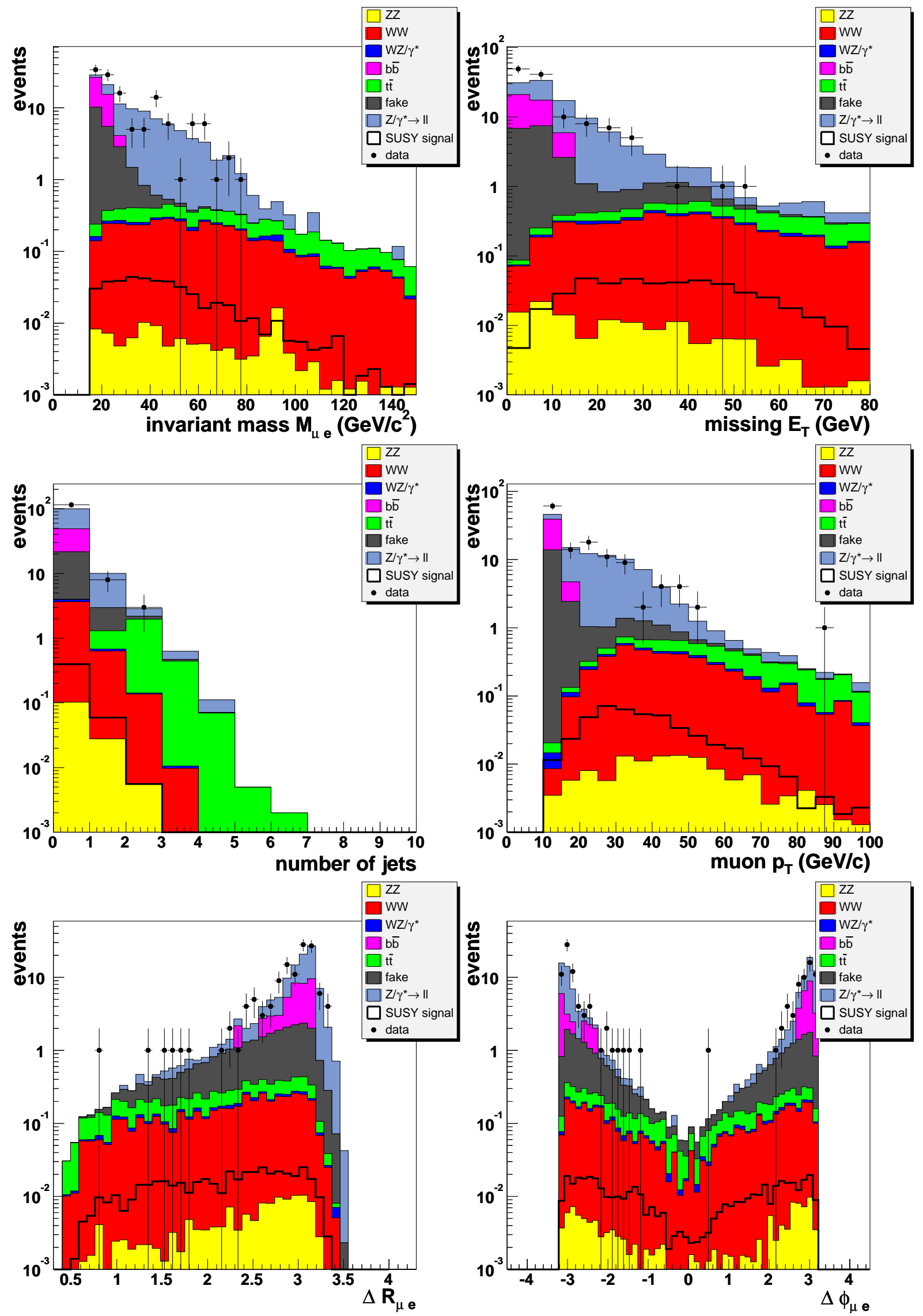

Figure 6.12: Opposite sign $\mu$ distributions: the whole invariant mass spectrum. 

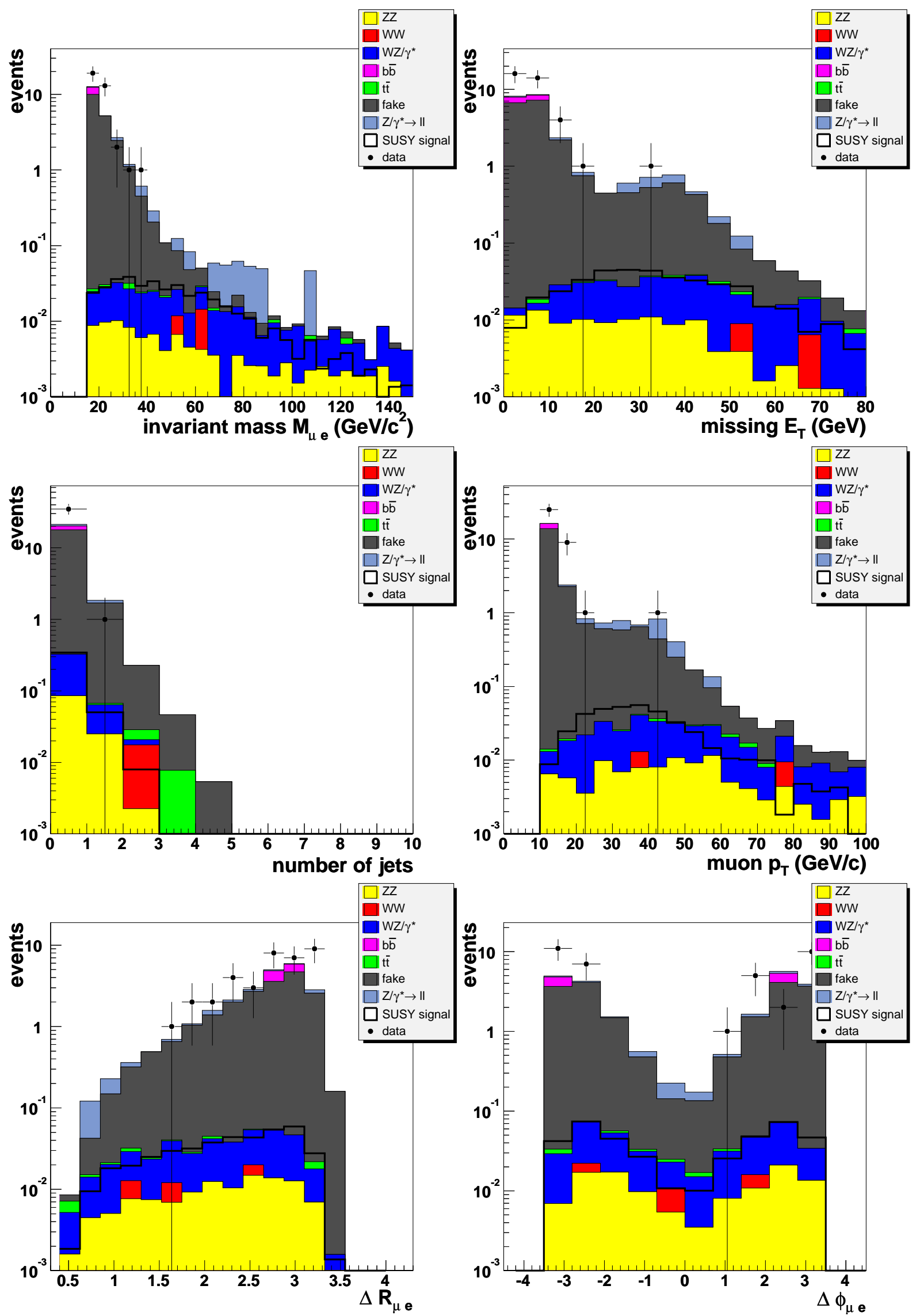

Figure 6.13: Same sign $\mu$ distributions: the whole invariant mass spectrum. 

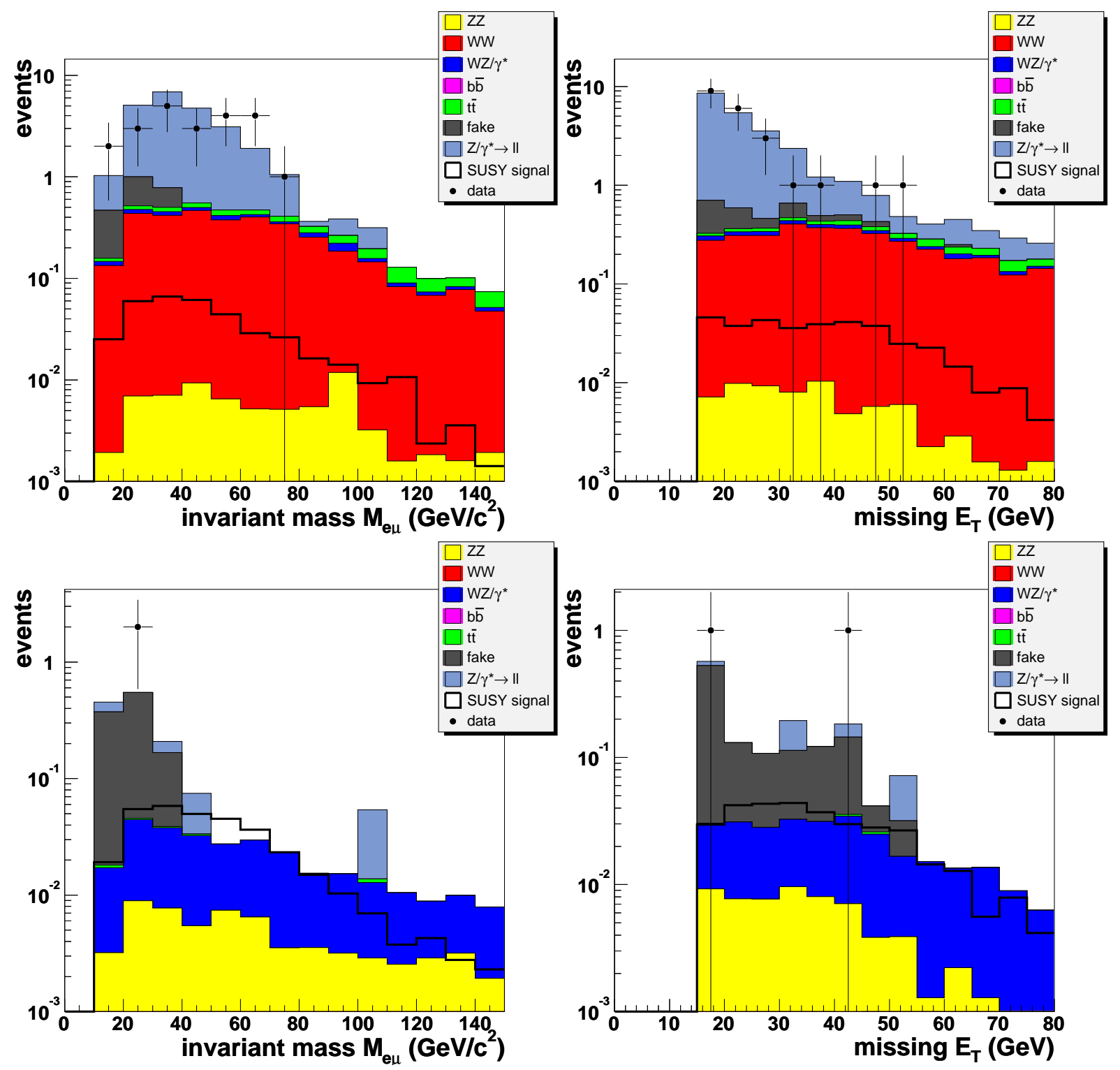

Figure 6.14: e $\mu$ distributions in control region $A+E$ : opposite sign and same sign in the top and bottom side respectively. 

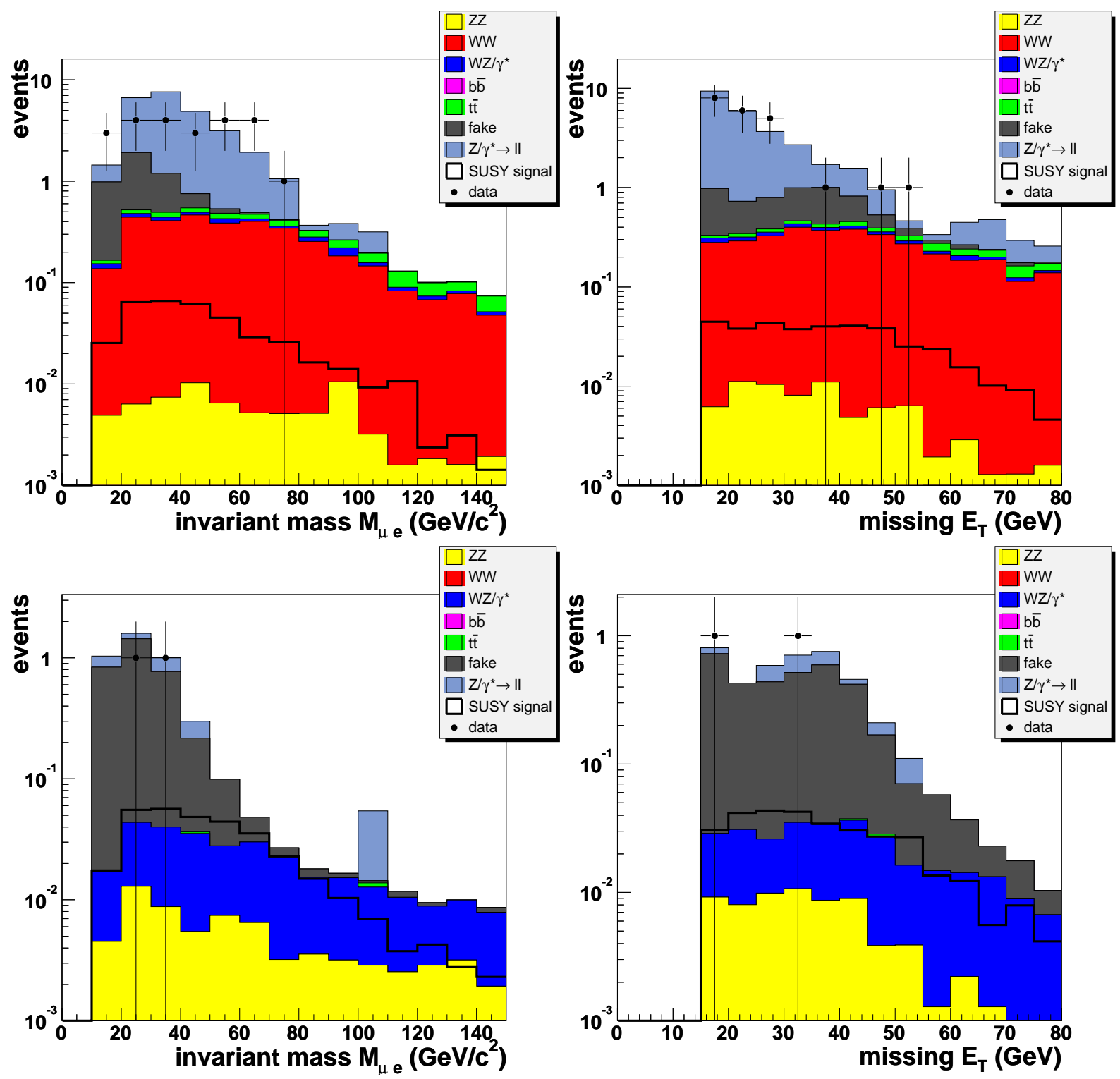

Figure 6.15: $\mu$ e distributions in control region $A+E$ : opposite sign and same sign in the top and bottom side respectively. 

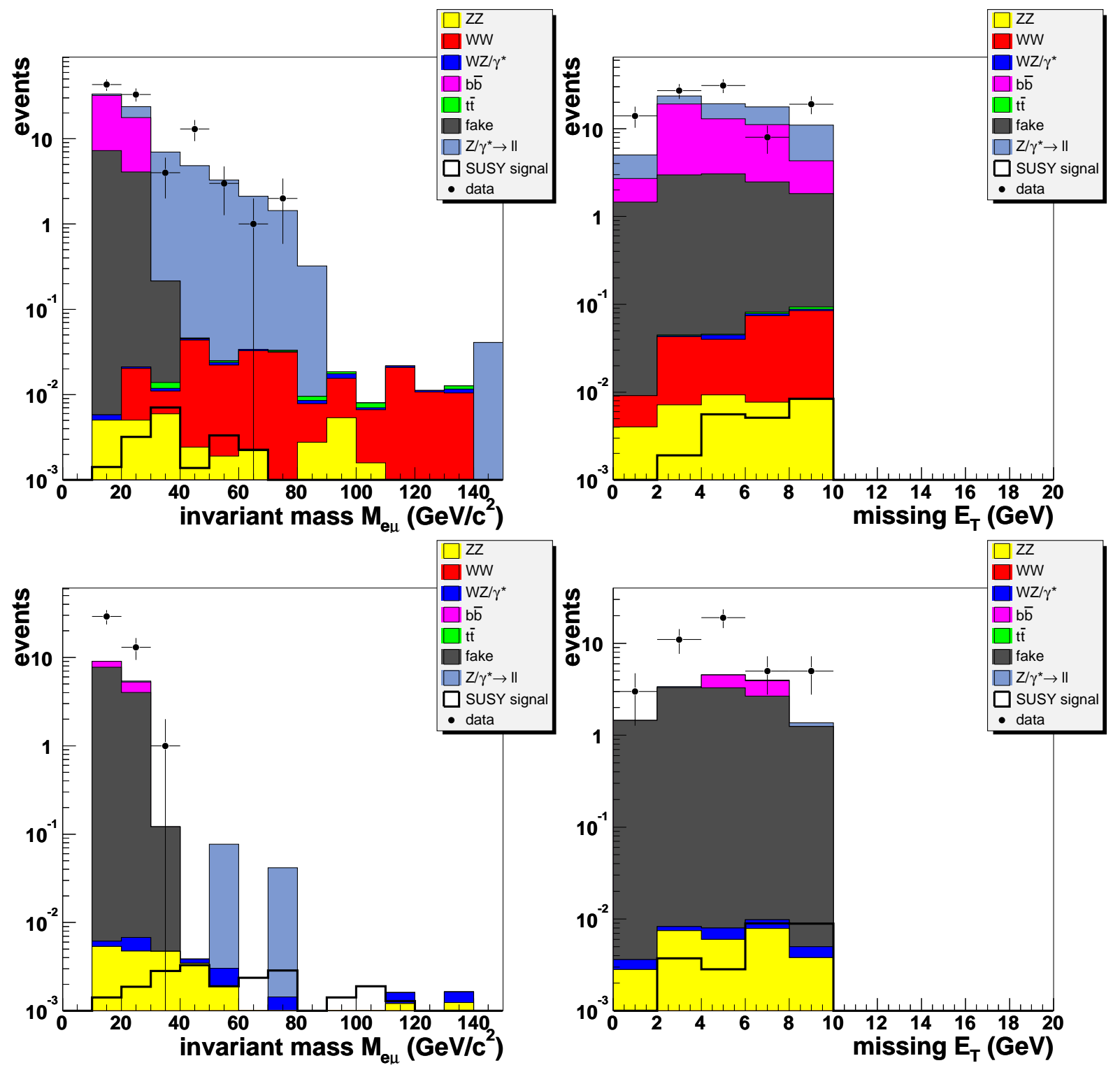

Figure 6.16: e $\mu$ distributions in control region $G+I$ : opposite sign and same sign in the top and bottom side respectively. 

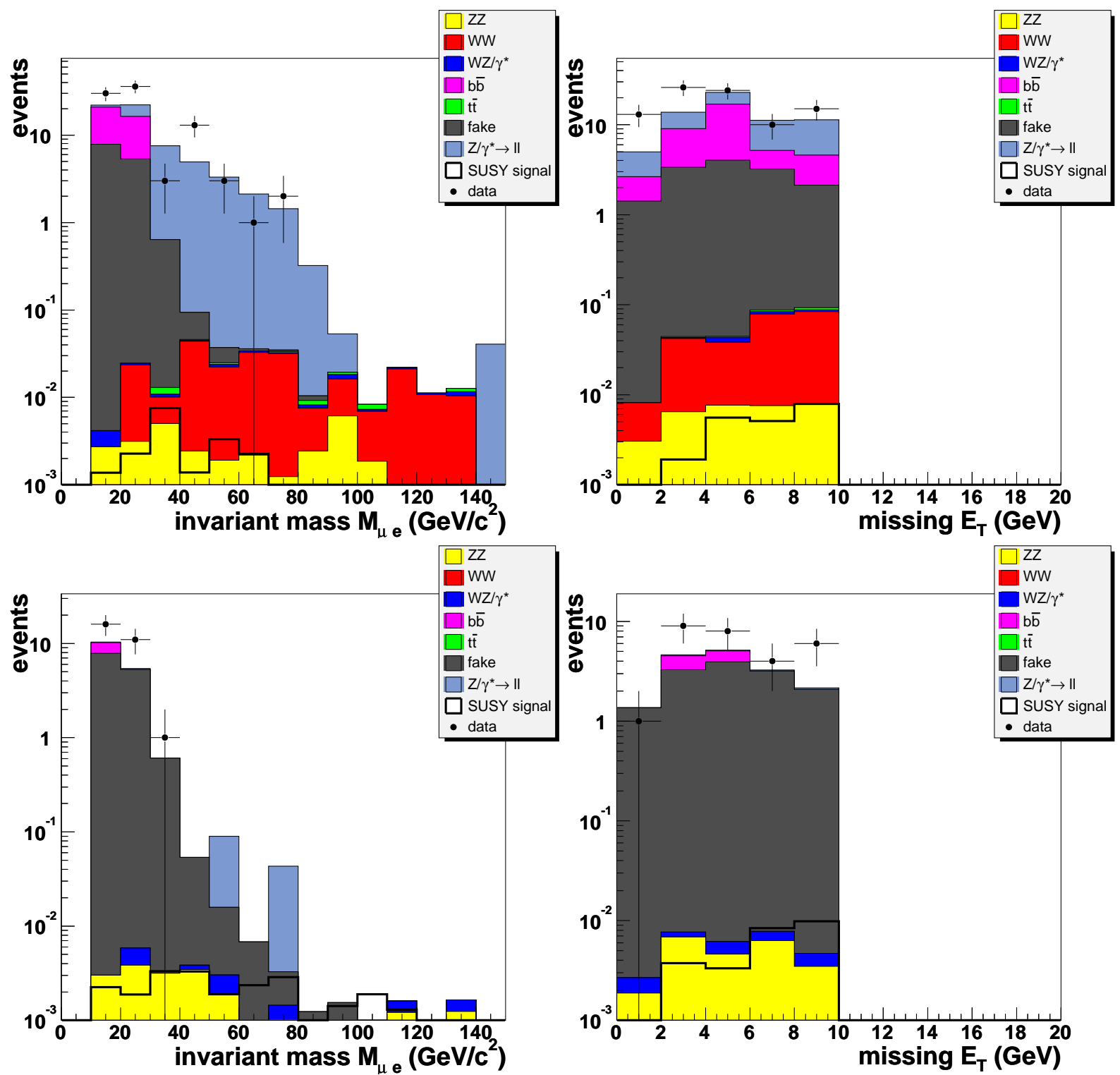

Figure 6.17: $\mu$ e distributions in control region $G+I$ : opposite sign and same sign in the top and bottom side respectively. 

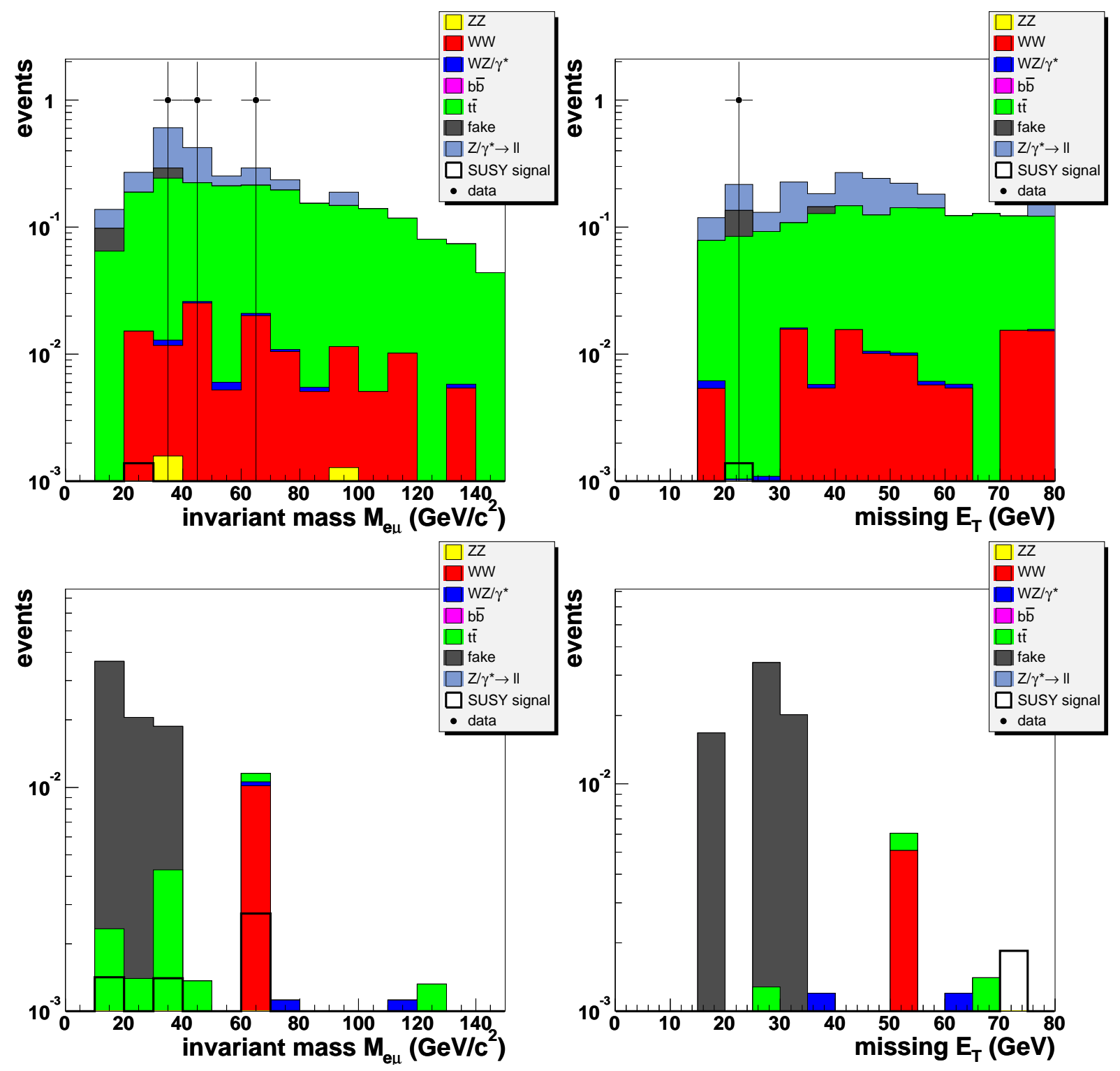

Figure 6.18: e $\mu$ distributions in control region A2+F: opposite sign and same sign in the top and bottom side respectively. 

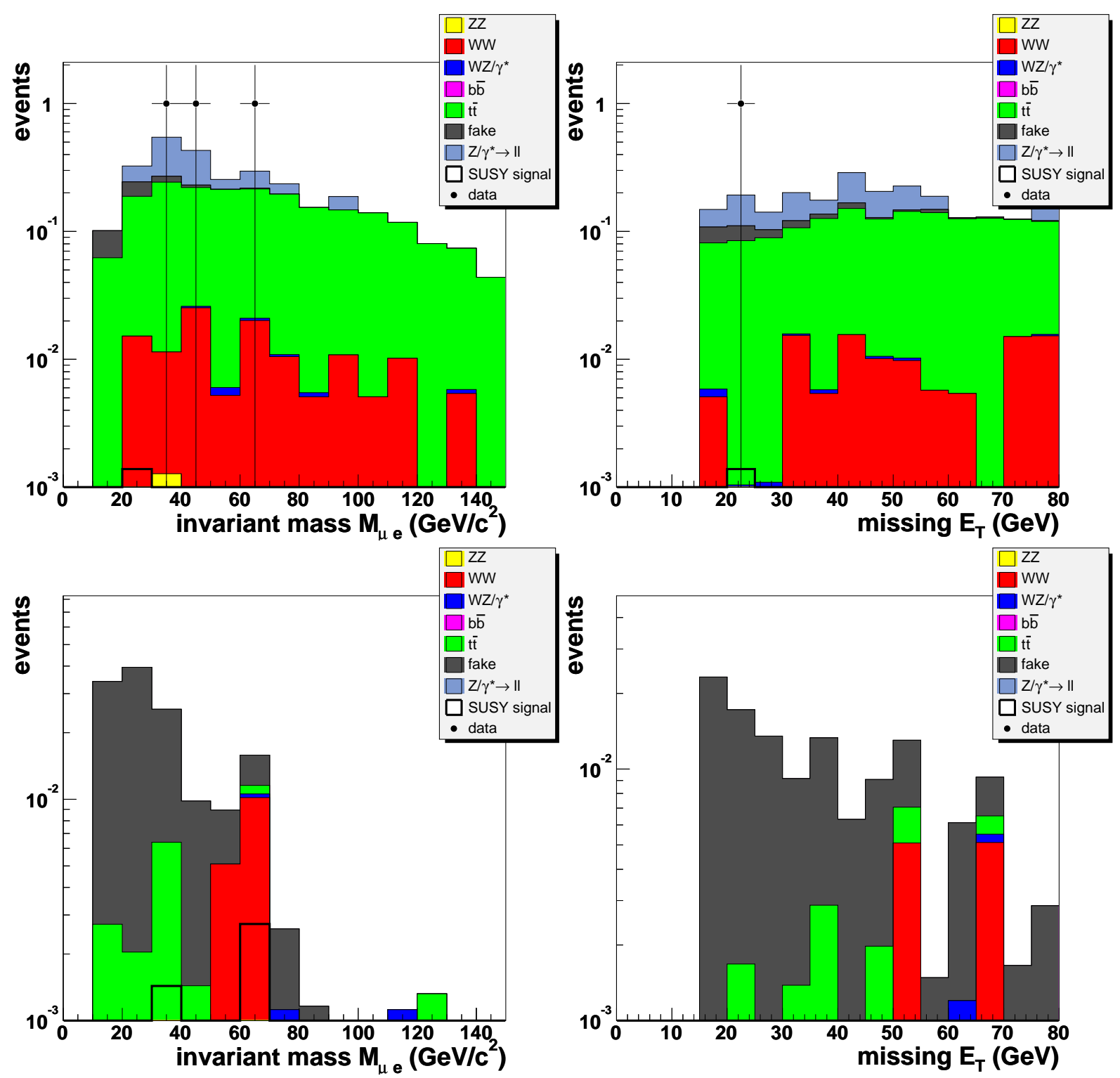

Figure 6.19: $\mu$ e distributions in control region A2+F: opposite sign and same sign in the top and bottom side respectively. 

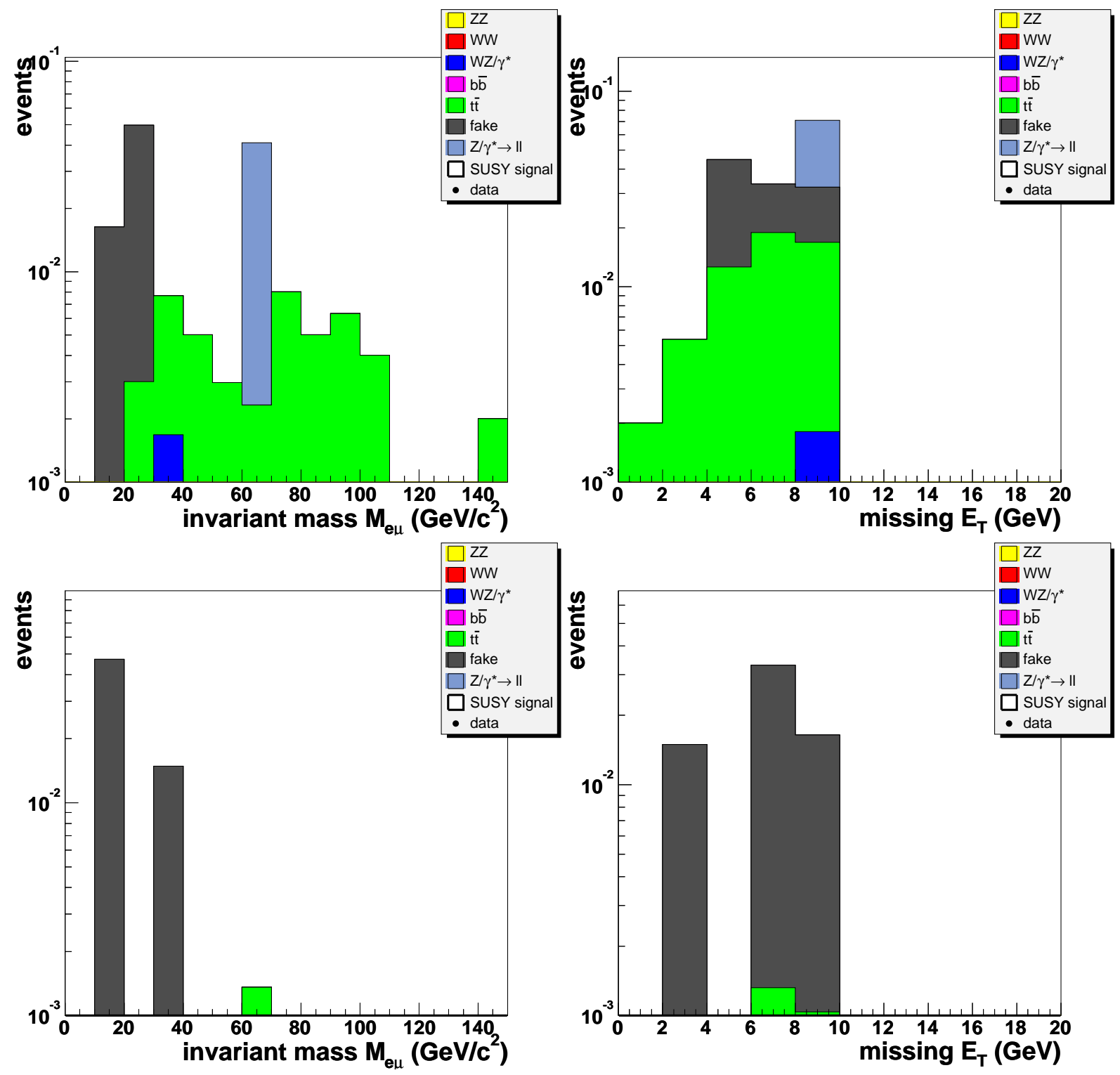

Figure 6.20: e $\mu$ distributions in control region $H+J$ : opposite sign and same sign in the top and bottom side respectively. 

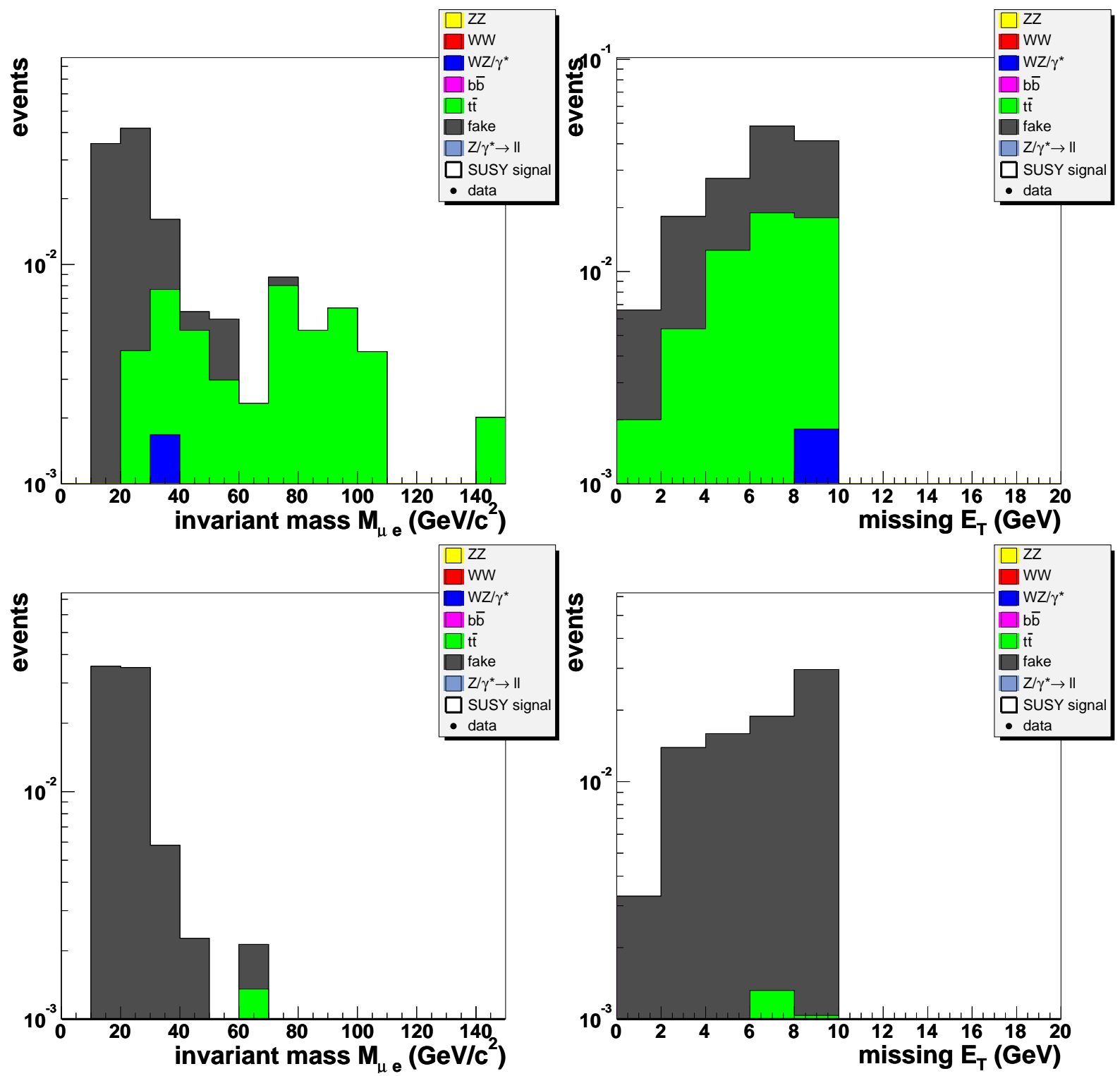

Figure 6.21: $\mu$ e distributions in control region $H+J$ : opposite sign and same sign in the top and bottom side respectively. 


\begin{tabular}{|l|c|c|c|c|}
\hline & $\mathrm{A}+\mathrm{E}$ & $\mathrm{G}+\mathrm{I}$ & $\mathrm{A} 2+\mathrm{F}$ & $\mathrm{H}+\mathrm{J}$ \\
\hline \hline Signal & $0.71356 \pm 0.00052$ & $0.04697 \pm 0.00013$ & $0.0132 \pm 0.0001$ & $0.00047 \pm 0.00001$ \\
Data & 24 & 142 & 3 & 0 \\
Total BG & $27.41028 \pm 0.23587$ & $91.24584 \pm 10.35184$ & $3.3885 \pm 0.0478$ & $0.22130 \pm 0.01028$ \\
\hline \hline$Z Z$ & $0.14589 \pm 0.00016$ & $0.06453 \pm 0.00011$ & $0.00671 \pm 0.00003$ & $0.00187 \pm 0.00009$ \\
$W^{ \pm} Z$ & $0.56422 \pm 0.00039$ & $0.02020 \pm 0.00007$ & $0.00912 \pm 0.00005$ & $0.00118 \pm 0.00009$ \\
$W^{ \pm} W^{ \pm}$ & $3.64916 \pm 0.01266$ & $0.21465 \pm 0.00307$ & $0.14686 \pm 0.00254$ & $0.00000 \pm 0.00000$ \\
$Z / \gamma^{*} \rightarrow l l$ & $20.34106 \pm 0.23553$ & $26.55227 \pm 0.26910$ & $0.83548 \pm 0.04773$ & $0.03873 \pm 0.01028$ \\
$t \bar{t}$ & $0.64127 \pm 0.00105$ & $0.01005 \pm 0.00013$ & $2.23934 \pm 0.00196$ & $0.05522 \pm 0.00031$ \\
$b \bar{b}$ & $0.00000 \pm 0.00000$ & $40.93522 \pm 10.34834$ & $0.00000 \pm 0.00000$ & $0.00000 \pm 0.00000$ \\
fakes & 2.06869 & 23.44893 & 0.15093 & 0.12430 \\
\hline
\end{tabular}

Table 6.3: Number of events in signal, data and background when selecting e $\mu$ events $(O S+S S)$.

\begin{tabular}{|l|c|c|c|c|}
\hline & $\mathrm{A}+\mathrm{E}$ & $\mathrm{G}+\mathrm{I}$ & $\mathrm{A} 2+\mathrm{F}$ & $\mathrm{H}+\mathrm{J}$ \\
\hline \hline Signal & $0.71294 \pm 0.00052$ & $0.04781 \pm 0.00014$ & $0.01314 \pm 0.00007$ & $0.00047 \pm 0.00001$ \\
Data & 25 & 116 & 3 & 0 \\
Total BG & $33.11854 \pm 0.24335$ & $80.92725 \pm 8.35337$ & $3.40699 \pm 0.04554$ & $0.22403 \pm 0.00031$ \\
\hline \hline$Z Z$ & $0.15413 \pm 0.00017$ & $0.05587 \pm 0.00010$ & $0.00545 \pm 0.00003$ & $0.00187 \pm 0.00002$ \\
$W^{ \pm} Z$ & $0.56077 \pm 0.00039$ & $0.02087 \pm 0.00008$ & $0.00754 \pm 0.00005$ & $0.00118 \pm 0.00002$ \\
$W^{ \pm} W^{ \pm}$ & $3.65242 \pm 0.01267$ & $0.21973 \pm 0.00311$ & $0.15197 \pm 0.00258$ & $0.00000 \pm 0.00000$ \\
$Z / \gamma^{*} \rightarrow l l$ & $21.65464 \pm 0.24302$ & $26.24040 \pm 0.26751$ & $0.75670 \pm 0.04543$ & $0.00000 \pm 0.00000$ \\
$t \bar{t}$ & $0.64568 \pm 0.00105$ & $0.01005 \pm 0.00013$ & $2.24183 \pm 0.00196$ & $0.05626 \pm 0.00031$ \\
$b \bar{b}$ & $0.00000 \pm 0.00000$ & $26.64609 \pm 8.34908$ & $0.00000 \pm 0.00000$ & $0.00000 \pm 0.00000$ \\
fakes & 6.45089 & 27.73424 & 0.24349 & 0.16472 \\
\hline
\end{tabular}

Table 6.4: Number of events in signal, data and background when selecting $\mu$ events $(O S+S S)$.

\subsubsection{Trilepton control regions}

The summary of trilepton events for different control regions is presented in Tables 6.5-6.6. In the trilepton selection, according to the analysis apporach, the signal region with high missing energy is kept blind in data. After selecting the third object, no data events are left in the other control regions, consistently with almost no SM background contributions. As anticipated in Section 6.4, the main backgrounds left in the signal region come from Drell-Yan and $W^{ \pm} Z$ processes.

\subsection{Summary and discussion}

The comparison of data and MC when selecting dimuon and dielectron events is within statistical uncertainties. On the other hand, comparing observed and expected events for the $e \mu$ channel shows that more work is needed before unblinding the signal box. More specifically: 


\begin{tabular}{|l|c|c|c|c|}
\hline & $\mathrm{A}+\mathrm{E}$ & $\mathrm{G}+\mathrm{I}$ & $\mathrm{A} 2+\mathrm{F}$ & $\mathrm{H}+\mathrm{J}$ \\
\hline \hline Signal MC & $0.14054 \pm 0.00023$ & $0.00774 \pm 0.00005$ & $0.00179 \pm 0.00003$ & $0.000000 \pm 0.00000$ \\
Data & BLIND & 0 & BLIND & 0 \\
Total MC BG & $0.11593 \pm 0.01026$ & $0.23713 \pm 0.02458$ & $0.00196 \pm 0.00004$ & $0.000916 \pm 0.00001$ \\
\hline \hline$Z Z$ & $0.02007 \pm 0.00006$ & $0.01371 \pm 0.00005$ & $0.00096 \pm 0.00001$ & $0.00092 \pm 0.00001$ \\
$W^{ \pm} Z$ & $0.05725 \pm 0.00013$ & $0.00182 \pm 0.00002$ & $0.00000 \pm 0.00000$ & $0.00000 \pm 0.00000$ \\
$W^{ \pm} W^{ \pm}$ & $0.00000 \pm 0.00000$ & $0.00000 \pm 0.00000$ & $0.00000 \pm 0.00000$ & $0.00000 \pm 0.00000$ \\
$Z / \gamma^{*} \rightarrow l l$ & $0.03861 \pm 0.01026$ & $0.22161 \pm 0.02458$ & $0.00000 \pm 0.00000$ & $0.00000 \pm 0.00000$ \\
$t \bar{t}$ & $0.00000 \pm 0.00000$ & $0.00000 \pm 0.00000$ & $0.00100 \pm 0.00004$ & $0.00000 \pm 0.00000$ \\
$b \bar{b}$ & $0.00000 \pm 0.00000$ & $0.00000 \pm 0.00000$ & $0.00000 \pm 0.00000$ & $0.00000 \pm 0.00000$ \\
\hline
\end{tabular}

Table 6.5: Number of events in signal, data and background when selecting e $\mu+l$ events $(O S+S S)$.

\begin{tabular}{|l|c|c|c|c|}
\hline & $\mathrm{A}+\mathrm{E}$ & $\mathrm{G}+\mathrm{I}$ & $\mathrm{A} 2+\mathrm{F}$ & $\mathrm{H}+\mathrm{J}$ \\
\hline \hline Signal MC & $0.13608 \pm 0.00023$ & $0.00774 \pm 0.00005$ & $0.00218 \pm 0.00003$ & $0.00000 \pm 0.00000$ \\
Data & BLIND & 0 & BLIND & 0 \\
Total MC BG & $0.11277 \pm 0.01026$ & $0.27331 \pm 0.02641$ & $0.00132 \pm 0.00004$ & $0.00092 \pm 0.00001$ \\
\hline \hline$Z Z$ & $0.01808 \pm 0.00006$ & $0.01532 \pm 0.00005$ & $0.00032 \pm 0.00001$ & $0.00092 \pm 0.00001$ \\
$W^{ \pm} Z$ & $0.05608 \pm 0.00012$ & $0.00222 \pm 0.00003$ & $0.00000 \pm 0.00000$ & $0.00000 \pm 0.00000$ \\
$W^{ \pm} W^{ \pm}$ & $0.00000 \pm 0.00000$ & $0.00000 \pm 0.00000$ & $0.00000 \pm 0.00000$ & $0.00000 \pm 0.00000$ \\
$Z / \gamma^{*} \rightarrow l l$ & $0.03861 \pm 0.01026$ & $0.25578 \pm 0.02641$ & $0.00000 \pm 0.00000$ & $0.00000 \pm 0.00000$ \\
$t \bar{t}$ & $0.00000 \pm 0.00000$ & $0.00000 \pm 0.00000$ & $0.00100 \pm 0.00004$ & $0.00000 \pm 0.00000$ \\
$b \bar{b}$ & $0.00000 \pm 0.00000$ & $0.00000 \pm 0.00000$ & $0.00000 \pm 0.00000$ & $0.00000 \pm 0.00000$ \\
\hline
\end{tabular}

Table 6.6: Number of events in signal, data and background when selecting $\mu$ e $l$ events $(O S+S S)$

1. because the biggest statistical uncertainty in MC samples regards the $b \bar{b}$ simulation, this sample needs to be considerably augmented;

2. the overlap between fakes and other backgrounds needs to be taken into account by matching offline leptons to parton level leptons in MC events. For instance Fig. 6.8 shows a peak in correspondance of the $Z$ peak which is probably due to this overlap, although no evidence of the $Z$ peak is expected in the $e \mu$ invariant mass distribution;

3. in SS events a larger number of data than MC events are obtained. Since fakes are expected to be the main background for SS events, this discrepancy could be an indication that this background is not fully understood. It should be noted, however, that due to its poor statistics, the $e \mu$ channel is more sensitive to fakes than other trilepton channels.

4. both SS and OS events show discrepancies between data and MC in the low missing transverse energy region $(<10 \mathrm{GeV})$ which need to be understood;

5. another possible source of discrepancies which needs to be studied, if the effect of the fake missing transverse energy which could come from energy mismeasurements. 


\subsection{Future plans}

When all the above issues are resolved, the analysis will augment the third lepton acceptance by including stubless muons (CMIO) and forward electrons. Moreover the total luminosity collected before 2005 shutdown, which correpsond to $\sim 700 \mathrm{pb}^{-1}$, will be included in the analysis. When backgrounds are understood in control regions, additional analysis cuts will be studied to improve the $S / \sqrt{B}$ ratio. Finally systematics will be calculated and the signal box will be unblinded, in order to determine the limit on the chargino mass.

Other SUSY DILEPTON trigger paths will then be included in order to further increase the acceptance of the first two leptons. 


\section{Bibliography}

[1] S. Dube et Al., Measuring Central Electron Trigger Efficiencies for 4 GeV Triggers, CDF internal note 7095, 2005.

[2] D. Bortoletto et Al., Trigger Efficiency for medium $p_{T}$ Dimuon Triggers in Run II, CDF internal note 7196, 2004.

[3] C. Hill et Al., Electron Identification in Offline Release 5.3, CDF internal note 7309, 2004.

[4] M. Griffiths et Al., Central Electron ID Efficiencies at Medium Energy, CDF internal note $7233,2004$.

[5] S. Dube et Al., Low Et electron ID efficiency and scale-factors using J/Psi, CDF internal note 7379, 2004.

[6] V. Martin, High-Pt Muon ID Cuts and Efficiencies for use with 5.3.1 Data and 5.3.3 $M C$, CDF internal note 7367, 2004.

[7] D. Bortoletto et Al., ID efficiency for intermediate $p T$ muons, CDF internal note $7197,2004$.

[8] Data Quality Monitoring web page, http://www-cdf.fnal.gov/internal/dqm/dqm.html.

[9] M. Gold et Al., Search for the chargino-neutralino production at the $\mu \mu+l$ decay channel at 312 $\mathrm{pb}^{-1}$ CDF internal note 8101, Feb 2006.

[10] D. Bortoletto et Al., Search for the associated production of chargino and neutralino in the final state with one muon and two additional leptons CDF internal note 8114, Mar 2006.

[11] S. Dube et Al., Search for Chargino-Neutralino Production in mSUGRA Model in a Di-electron + Track Channel CDF internal note 8098, Feb 2006.

[12] M. Griffiths et Al., Search for associated production of chargino and neutralino in trilepton final state using the high-p $p_{T}$ electron trigger CDF internal note 7868, Sep 2005. 



\section{Conclusions}

The work presented in this thesis regards the search for the associated production of chargino and neutralino characterized by the presence of three leptons plus two LSPs and a neutrino in the final state. More specifically, the analysis focuses on final states with one electron one muon and an additional third lepton. Events are collected by means of a dilepton trigger, specifically designed for such supersymmetric searches.

This channel is particularly challenging because of its poor statistics which make it more sensitive to fake lepton contamination than other leptonic channels. Another background which becomes important in the lepton $p_{T}$ range considered is the heavy flavor production which is shown to be reduced by requiring a minimal amount of missing energy in the event.

The search has been developed as a blind analysis which requires that one understand Standard Model backgrounds before looking at the signal region.

The analysis infrastructure has been developed and progress has been made towards an understanding of Standard Model backgrounds. However some particulars still need to be fully understood before unblinding. To this end more heavy flavor Monte Carlo is needed to reduce the statistical error associated with it. In addition, discrepancies between data and standard model prediction at low missing transverse energy and a better understanding of fake contribution is needed. Successively the analysis will be extended to include further triggers and to the entire available data set. This thesis should be regarded as an account of work in progress. 



\section{Appendix A}

\section{SUSY DILEPTON Trigger Monitoring}

\section{A.1 Motivation}

Monitoring the performance of a trigger is needed in order to assure the correct behaviour of the trigger, to detect problems, suggest possible remedies and to keep track of trigger changes. Changes in the conditions of the subdetectors involved in the trigger shows up as discontinuities while changes in the trigger background which influence the trigger cross section usually show up as continuous trends. Stability (or continuity) of a suitable variable is therefore the first indicator in evaluating trigger performance.

Trigger stability is also a simple and good criterion for judging the quality of the events collected. The same variable can also be used to establish whether a run should be included in the "good run list", i.e. a list of runs considered good for further usage, in particular for physics analysis.

The monitoring technique developed for the SUSY DILEPTON trigger, in line with the trigger strategy outlined in [1], is described in this appendix and the consequent good run list is defined.

Data collected from February 2002 till August 2004 which correspond to an integrated luminosity of $\sim 380 \mathrm{pb}^{-1}$ are considered. A preliminary requirement is that runs must have all subdetectors of interest marked as good (table A.1), according to the Data Quality Monitoring Good Run list v7 [3]. This preselection does not explicitely require that any of the muon chambers be good so that to make it possible to recover part of the luminosity that would be otherwise rejected if the muon chambers are marked as bad.

\begin{tabular}{l|l}
\hline \hline ONLINE bits & CLC, L1T, L2T, L3T, CAL, COT \\
OFFLINE bits & COT, CAL \\
\hline \hline
\end{tabular}

Table A.1: ONLINE and OFFLINE bits required to be good for each run considered in this study.

The trigger strategy described in [1] and Chapter 3.2.1 consists in defining standardized lepton "types" and in calculating trigger efficiencies for each lepton type involved in the trigger. In conformity with this approach, monitoring of the SUSY DILEPTON trigger was 
developed for each of the six lepton types involved in the trigger: three electrons and three muons (see Tab. A.2).

\begin{tabular}{l|l|l}
\hline \hline Lepton type & L1 and L2 Trigger & Lepton type definition \\
\hline \hline CEM4 & L1_CEM4_PT4 \&\& & central electron with $p_{T}>4 \mathrm{GeV} / \mathrm{c}$ \\
& L2_CEM4_PT4_CES2(3) & \\
\hline CEM8 & L1_CEM8_PT8 \&\& & central electron with $p_{T}>8 \mathrm{GeV} / \mathrm{c}$ \\
& L2_CEM8_PT8_CES2(3) & \\
\hline PEM8 & L1_EM8 \&\& L2_PEM8 & forward electron with $p_{T}>8 \mathrm{GeV} / \mathrm{c}$ \\
\hline CMU4 & L1_CMU1.5_PT1.5 & central muon with $p_{T}>4 \mathrm{GeV} / \mathrm{c}$ (at Level 3) \\
\hline CMUP4 & L1_CMUP6_PT4 & $\begin{array}{l}\text { central muon with } p_{T}>4 \mathrm{GeV} / \mathrm{c} \\
\text { also using the outer CMP muon chamber. }\end{array}$ \\
\hline CMX4 & L1_CMX1.5_PT2_CSX & forward muon with $p_{T}>4 \mathrm{GeV} / \mathrm{c}$ (at Level 3) \\
\hline \hline
\end{tabular}

Table A.2: Standard lepton "types" are defined. The trigger primitives indicated in the middle column correspond to the trigger requirements at Level 1 and 2 for each lepton type.

\section{A.2 Monitoring Variable Definition}

The first step is to choose a suitable monitoring variable: it should give information about the quality of the events collected for each lepton trigger type. First events are classified according to the trigger path they have been collected by. Then, the offline information is used to check the lepton content of each event. Given that each of the trigger paths requires two leptons, these two leptons will be referred as lepton $\mathrm{A}$ and lepton $\mathrm{B}$ for each path, where A and B also refer to leptons of the same type. For each trigger path four quantities are considered:

- $D_{A}$ : number of events collected by the trigger path that fulfill the standard definition of lepton A at trigger level;

- $N_{A}$ : number of $D_{A}$ events that have lepton A reconstructed at offline level;

- $D_{B}$ : number of events collected by the trigger path that fulfill the standard definition of lepton B at trigger level;

- $N_{B}$ : number of $D_{B}$ events that have lepton B reconstructed at offline level.

Then two monitoring variables are defined:

- $R_{A}=\frac{N_{A}}{D_{A}}$

- $R_{B}=\frac{N_{B}}{D_{B}}$.

A lepton is considered as reconstructed at offline level if it passes the identification requirements described in 4.4. For each lepton type, the events that contribute to $R$ (numerator and denominator) are required to fulfill the corresponding standard lepton definition at 
trigger level and since a trigger bit corresponding to the single lepton is not always available, the trigger behaviour for single lepton is emulated by using a Level 1 and Level 2 trigger simulation, a code which reproduces the trigger decision.

Because higher values of $R$ correspond to a higher quality of the collected events $R$ can be thought as a sort of purity : $R$ is closer to 1 the more events with a corresponding lepton at trigger level have this lepton reconstructed at offline level. By construction there are several values of $R$ for each lepton type, each value obtained from a different trigger path. A priori, no significant differences between these different values are expected. In addition $R$ distributions are also expected to be stable in time.

$R$ distributions for each trigger path are shown in Fig. A.1 A.2 A.3 A.4 A.5 A.6. These plots demonstrate the utility of this monitoring technique: $R$ is seen to be locally stable in time and all visible steps are correlated to changes in the trigger (table A.3).

\begin{tabular}{|l|l|l|}
\hline \hline CHANGE & TRIGGER PATH & RUN \\
\hline \hline $\begin{array}{l}\text { no L2_TWO_CEM4_PT4_CES2 bit available } \\
\text { for standard lepton definition before }\end{array}$ & DIELECTRON_CENTRAL_4 & $\sim 145000$ \\
\hline $\begin{array}{l}E_{T} \text { estimated using the track associated } \\
\text { to the electron after this run }\end{array}$ & paths involving CEM4 & $\sim 164000$ \\
\hline code bug before this run & CEM8_PEM8 & $\sim 150000$ \\
\hline $\begin{array}{l}\text { CSX scintillator added at L1 } \\
\text { after this run }\end{array}$ & paths involving CMX4 & $\sim 154000$ \\
\hline \hline
\end{tabular}

Table A.3: Changes in trigger table related to steps in $R$ distributions. Starting from the left-hand side, the first column shows the trigger table change, the second column indicates which trigger paths are affected and the third column displays the first run in which the change was implemented in the trigger table. The CEM8_PEM8 path code bug was such that only one of CEM8 and PEM8 was required at trigger level. So far as CMX paths are concerned, the step in the $R$ distribution is only clearly visible for the CEM4_CMX4 trigger path.

The remaining sections are dedicated to investigating the use of this monitoring technique in defining a good run list. 

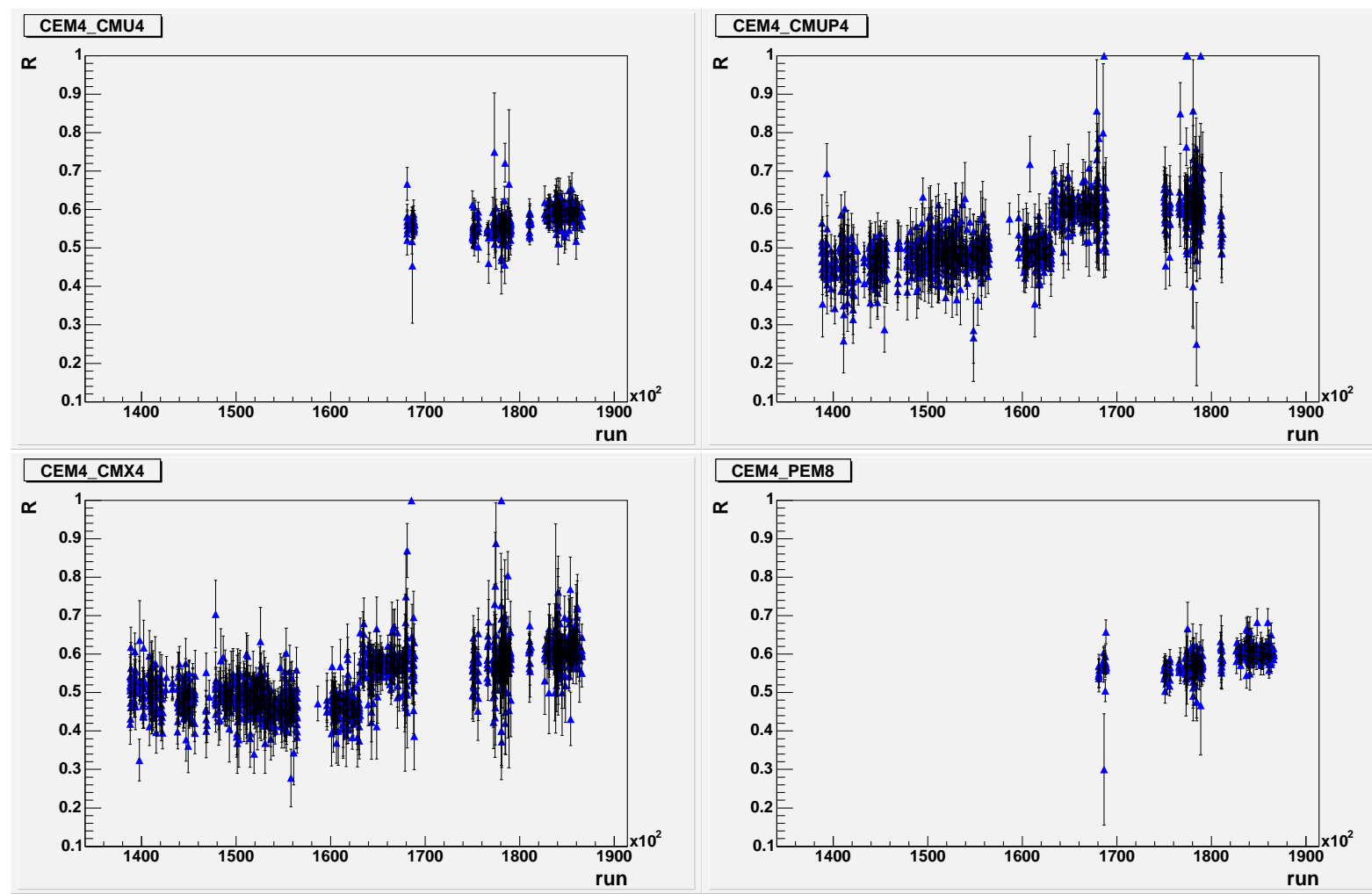

\section{CEM4_CEM4}

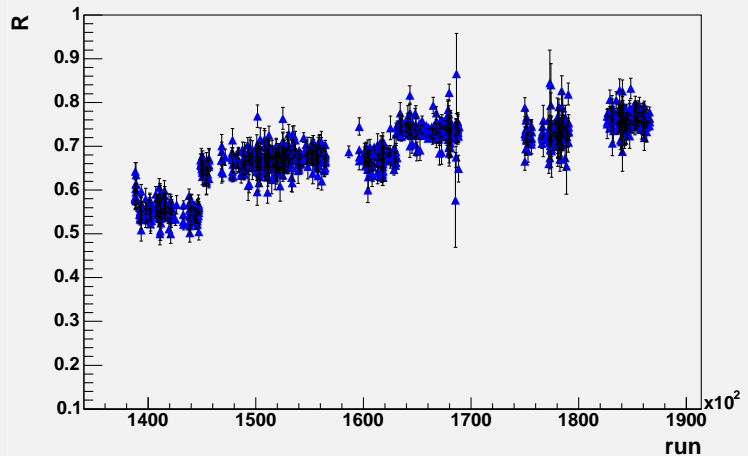

Figure A.1: $R$ distribution for CEM4 lepton type for each trigger path involving CEM4. 

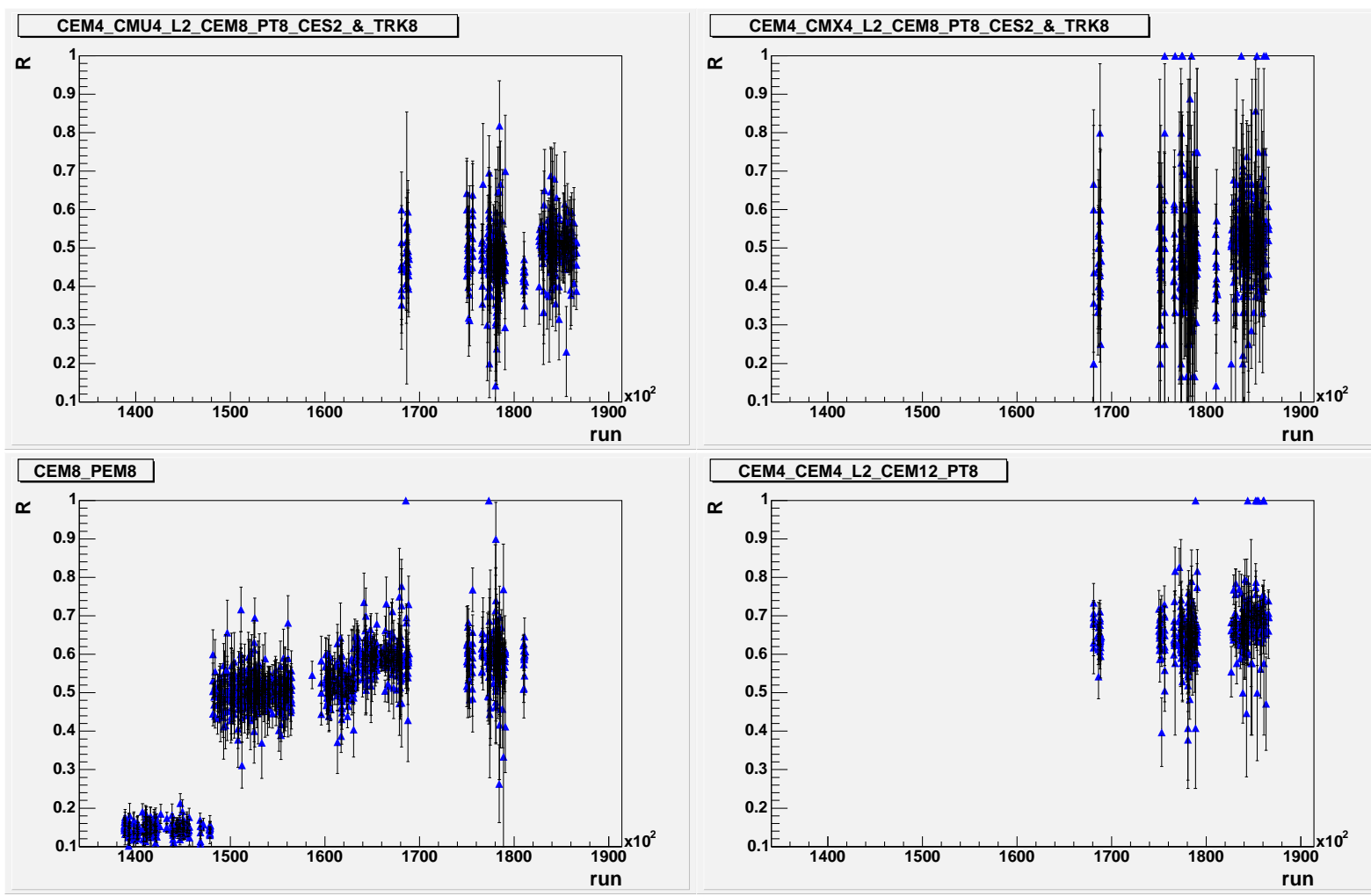

\section{CEM4_CEM4_L2_CEM12_PT8}

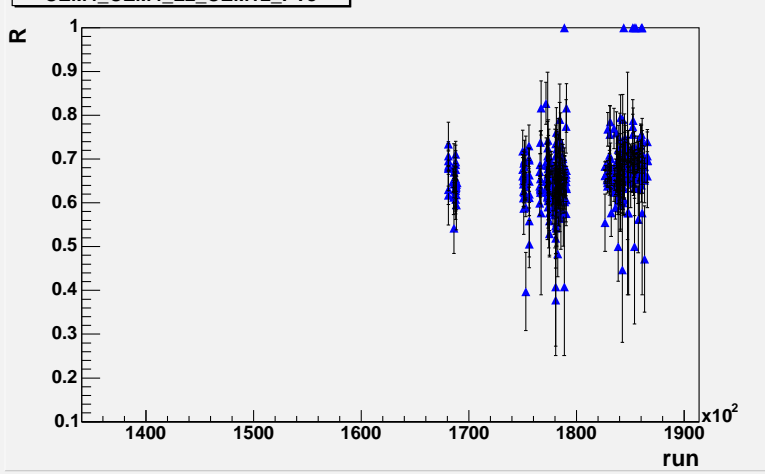

CEM4_CEM4_L2_CEM8_PT8_CES2_\&_TRK8

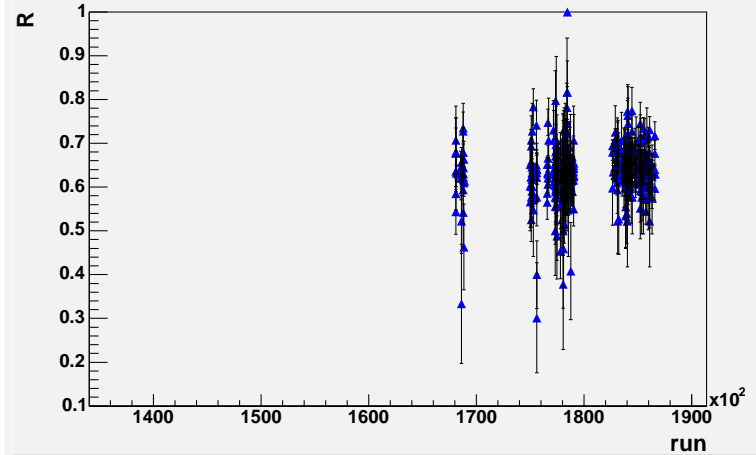

Figure A.2: $R$ distribution for CEM8 lepton type for each trigger path involving CEM8. 

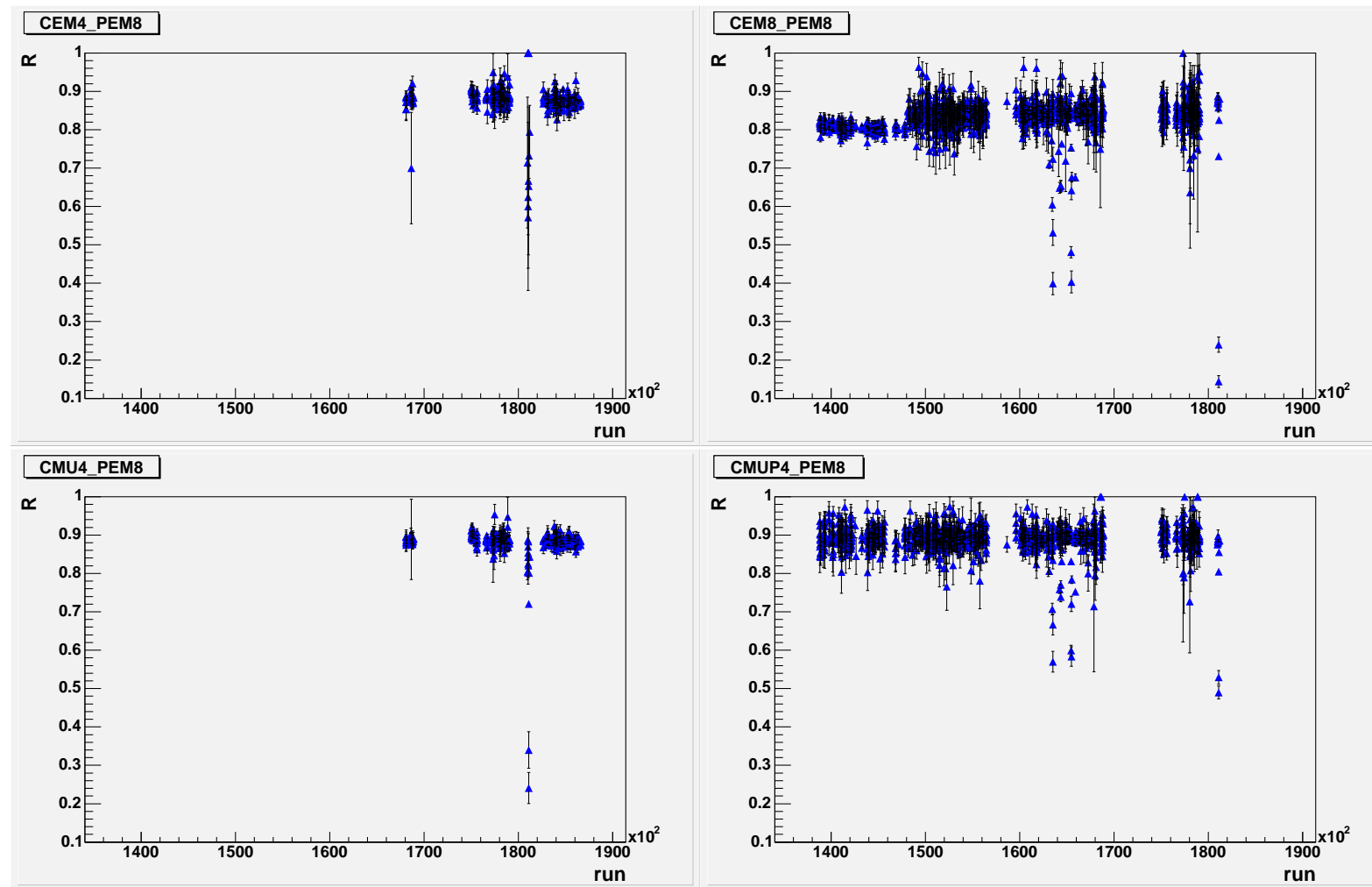

CMX4_PEM8

๓

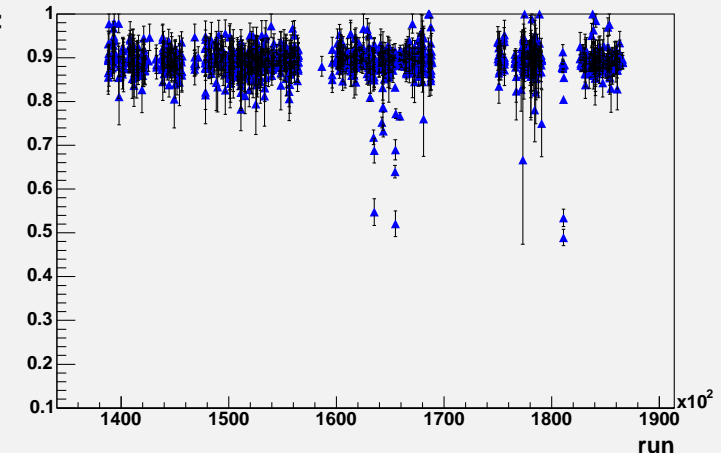

Figure A.3: $R$ distribution for PEM8 lepton type for each trigger path involving PEM8. 

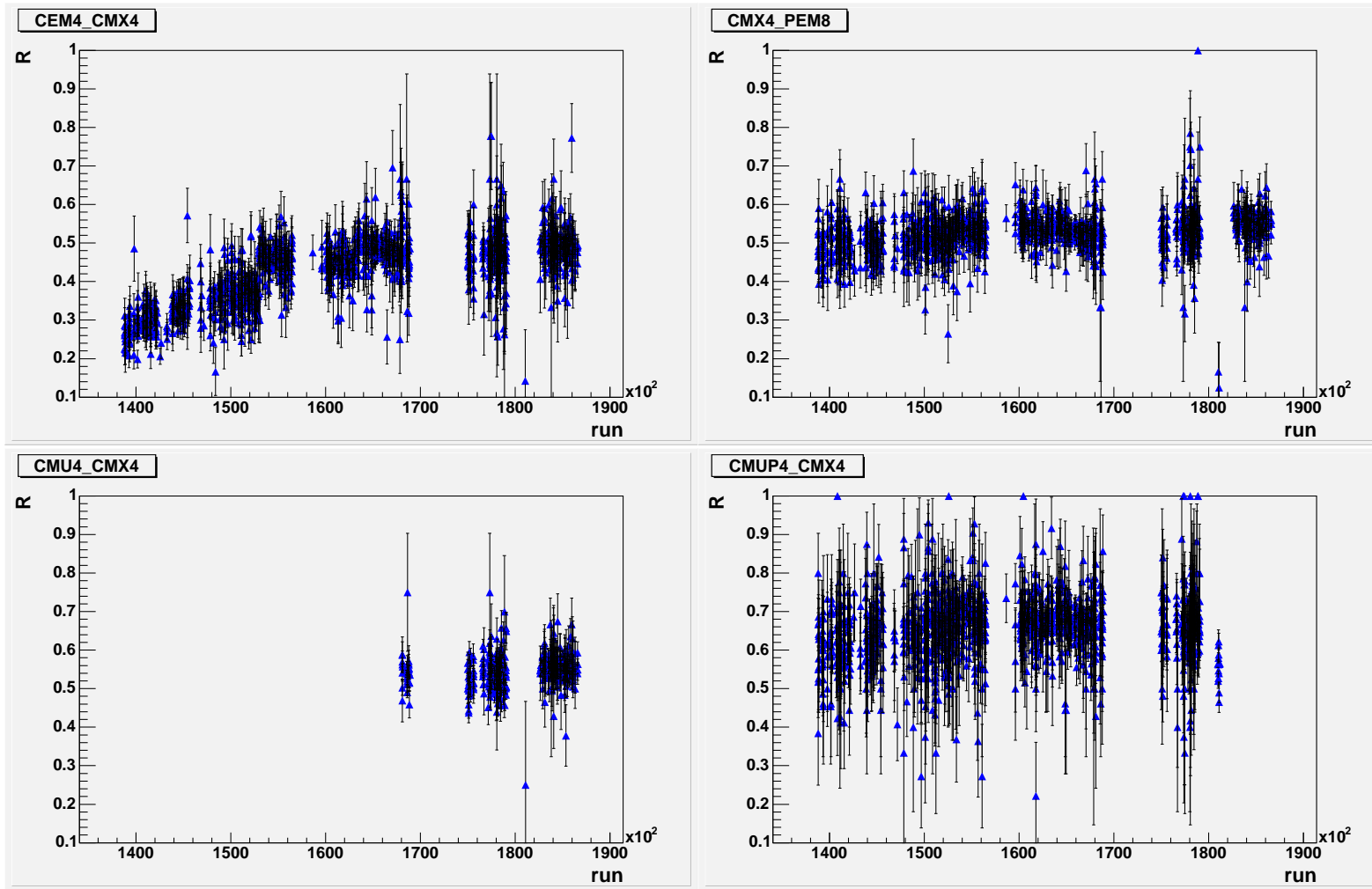

Figure A.4: $R$ distribution for $C M X_{4}$ lepton type for each trigger path involving $C M X_{4}$.
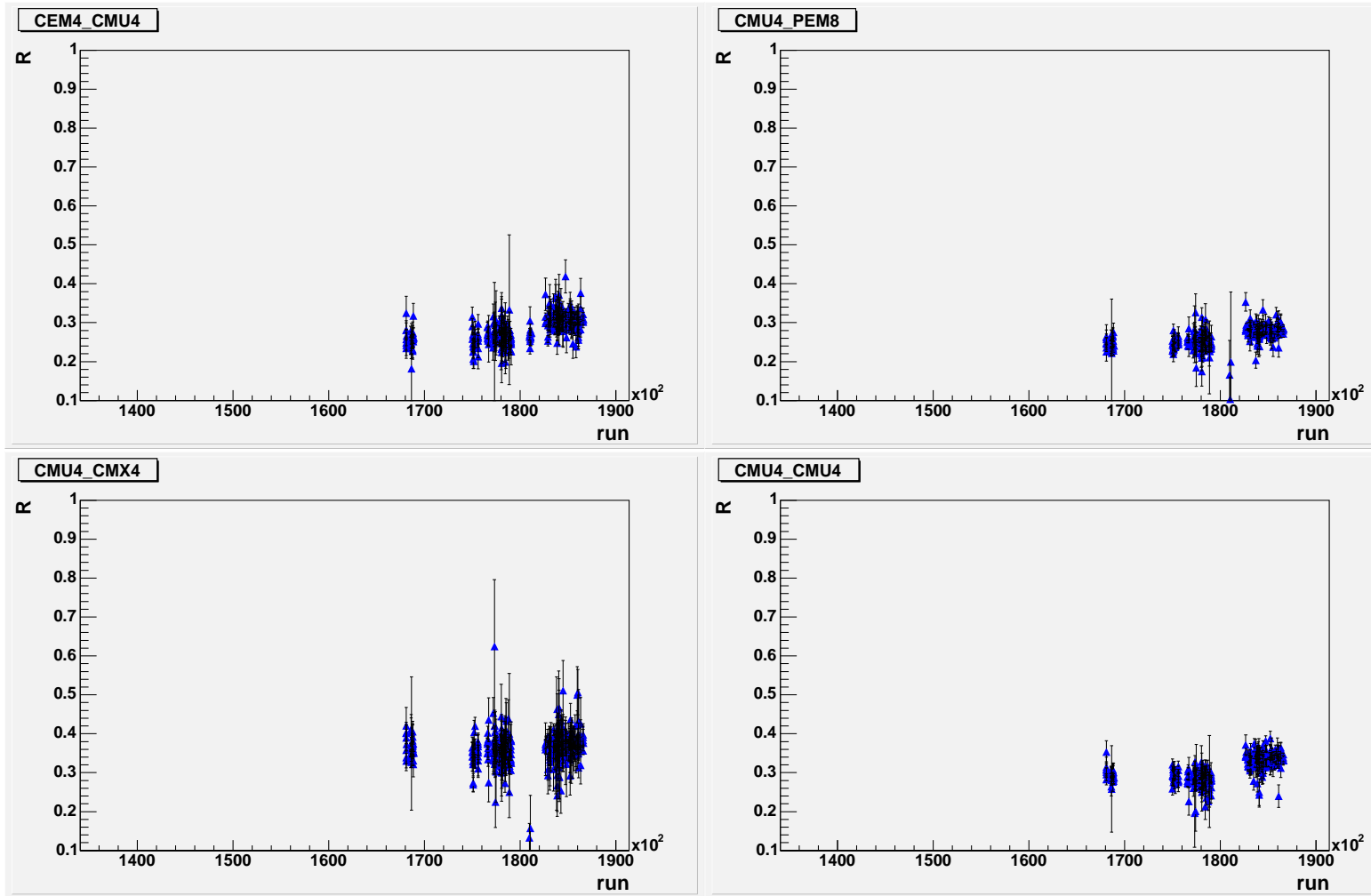

Figure A.5: $R$ distribution for $C M U 4$ lepton type for each trigger path involving $C M U 4$. 

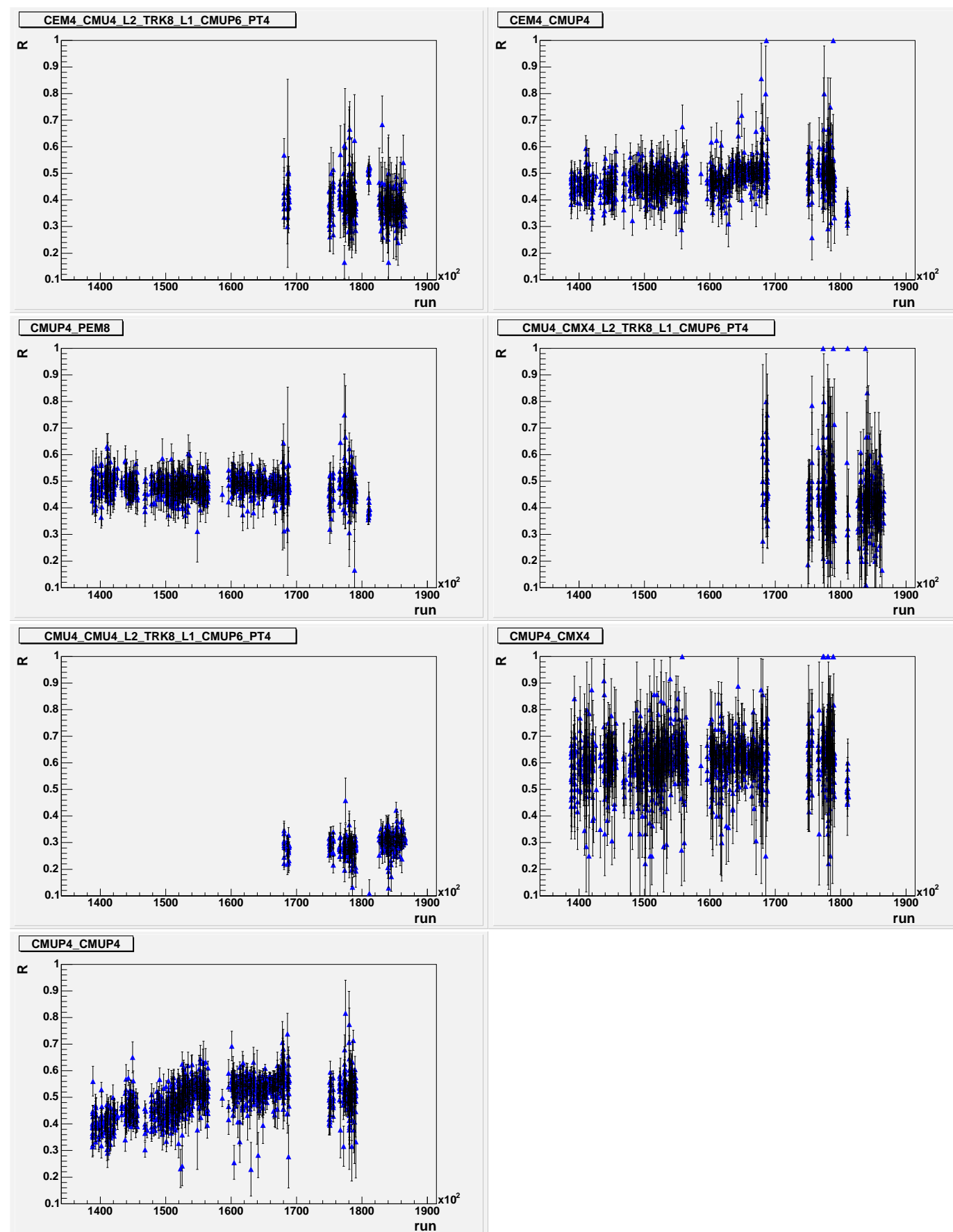

Figure A.6: $R$ distribution for CMUP 4 lepton type for each trigger path involving CMUP4. 


\section{A.3 Suspect Run Definition}

After choosing the monitoring variable a suspect run's quality is evaluated on the basis of two criteria: first, for each run and for each lepton type, $R$ values from different trigger paths are checked for significant differences; second, the stability of $R$ is verified.

A a suspect run is then defined as follows:

- STEP 1. For each run and for each lepton object, the weighted average of $R$ values coming from different trigger paths is calculated and called $R_{\text {lepton }}$. The run is then flagged as suspect if

$$
\left|R-R_{\text {lepton }}\right|>10 \sigma
$$

for at least one trigger path, where $R$ if the value of $R$ for each specific trigger path and where $\sigma$ is the statistical error associated to $R$.

- STEP 2. For each run, local averages $\left\langle R_{\text {lepton }}>\right.$ of the 20 runs before and after the run considered are calculated. The run is flagged as suspect if

$$
\left|R_{\text {lepton }}-<R_{\text {lepton }}>\right|>5 \sigma
$$

for both before/after averages, where $\sigma$ is the statistical error associated to $R_{\text {lepton }}$. Two different local averages are considered in order to keep into account steps in distributions.

The choice of $10 \sigma$ and $5 \sigma$ in STEP 1 and 2 respectively was dictated by the need, because of different cross sections, to take into account the different statistics of events collected by different trigger paths for a given amount of luminosity. In Fig. A.7 and A.8 the weighted average of $R$ calculated for each lepton type is shown before and after subtracting suspect runs.

\section{A.4 Good Run list on the basis of $\mathrm{R}$}

\begin{tabular}{l|l|l}
\hline \hline LEPTON TYPE & STEP1 $\left(\mathrm{pb}^{-1}\right)$ & STEP2 $\left(\mathrm{pb}^{-1}\right)$ \\
\hline CEM4 & 0.3 & 0 \\
CEM8 & 0.2 & 1.1 \\
PEM8 & 0.6 & 5.1 \\
CMX4 & 20.7 & 4 \\
CMU4 & 0.2 & 0.7 \\
CMUP4 & 1.2 & 0 \\
\hline TOTAL & 22.0 & 10.3 \\
\hline \hline
\end{tabular}

Table A.4: Suspect run luminosity in $\mathrm{pb}^{-1}$ for each lepton type at STEP1 and STEP2 defined in A.3. The COT compromised period is neglected. 

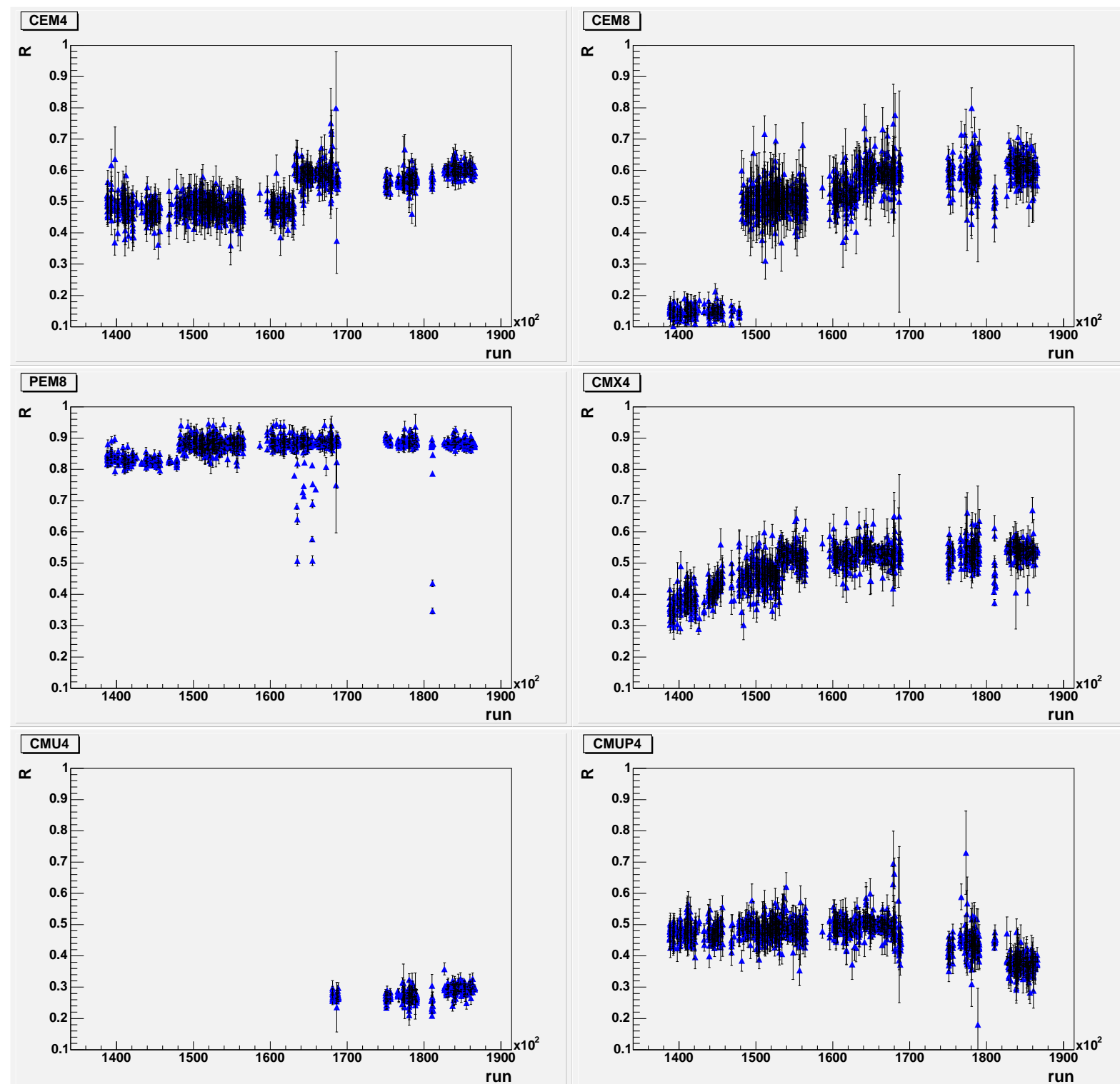

Figure A.7: Distributions of $R$ weighted average per each lepton type. 

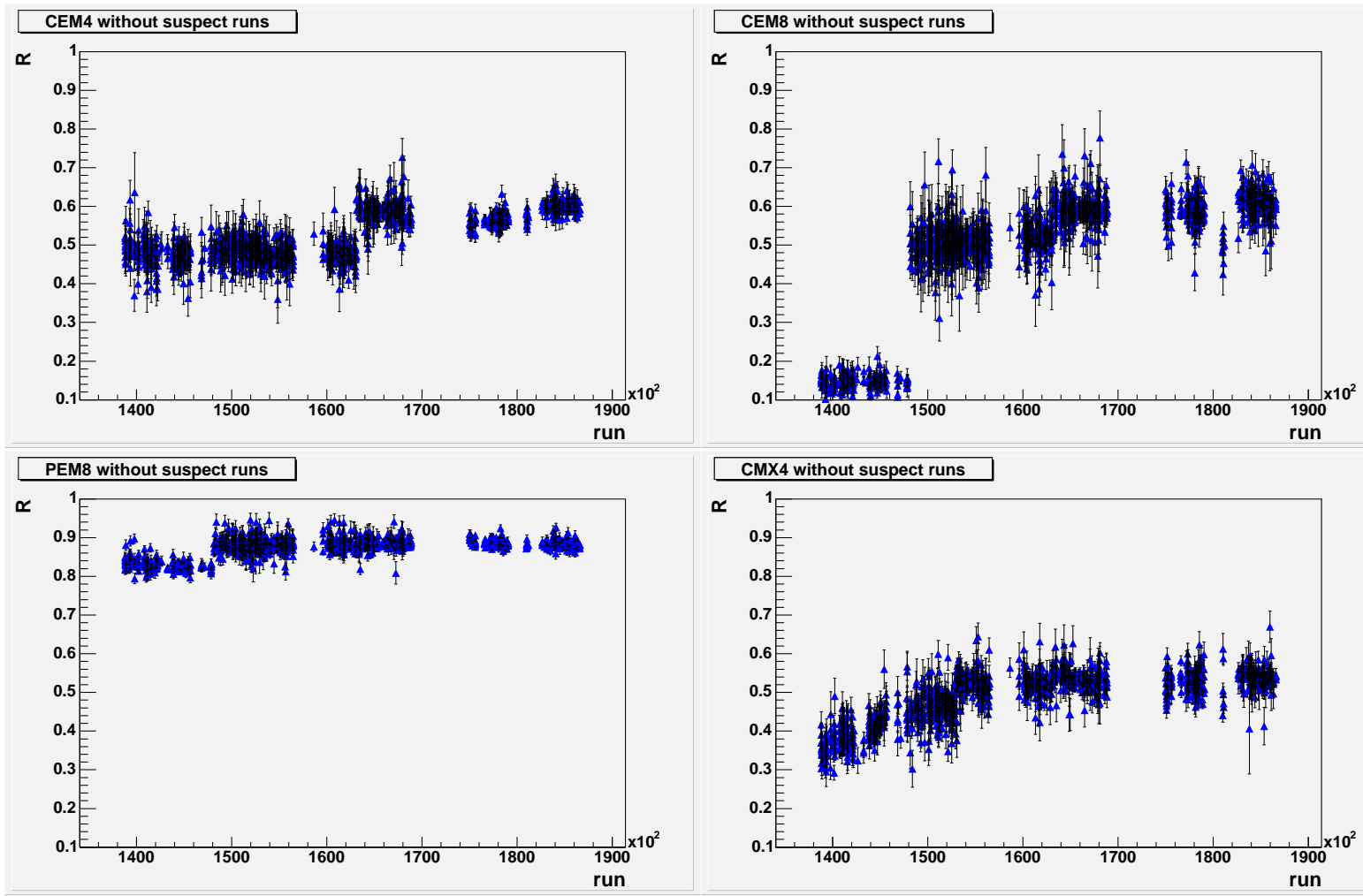

CMX4 without suspect runs

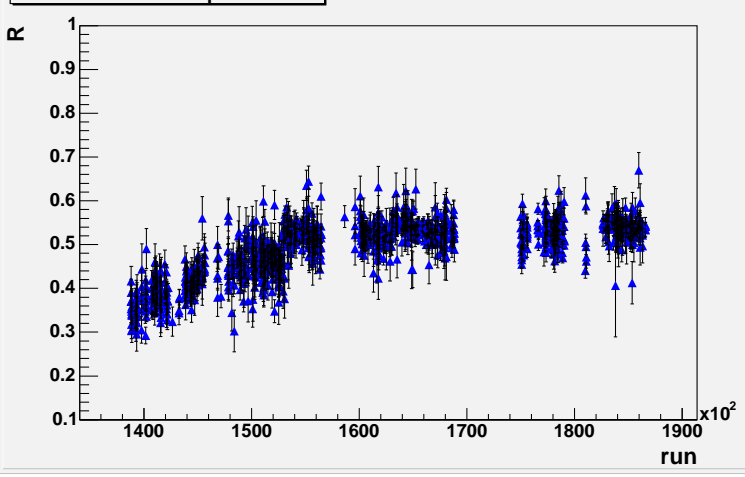

CMU4 without suspect runs
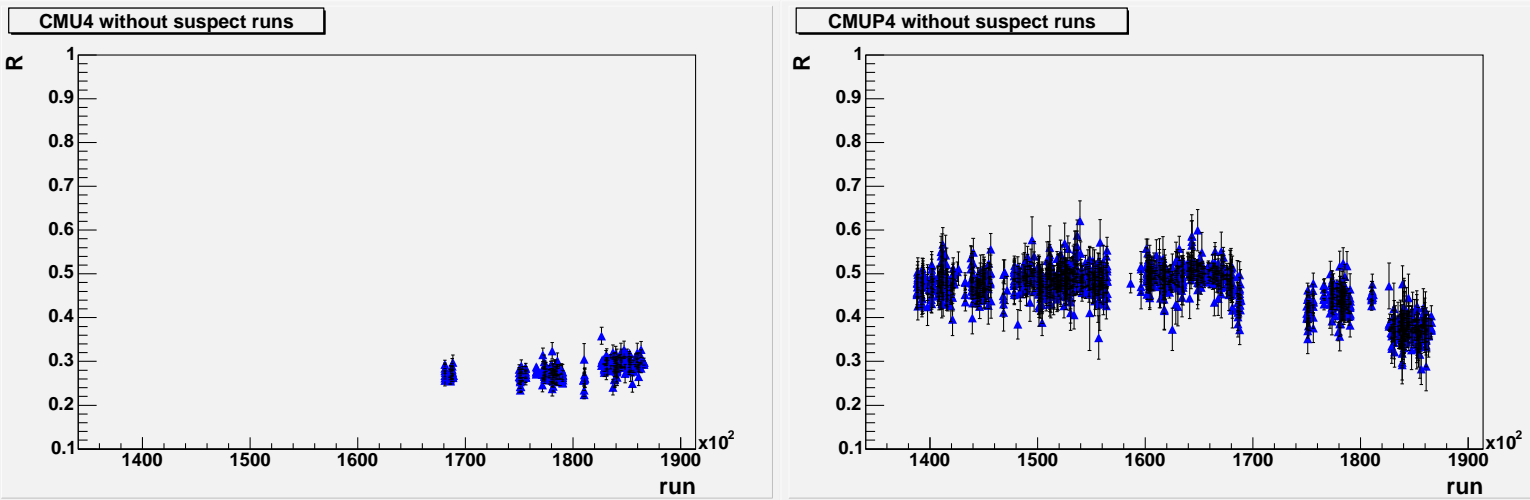

Figure A.8: Distributions of $R$ weighted average per each lepton type after subtracting suspect runs. 
In table A.4 the suspect run luminosity calculated according to the definition given in A.3 is reported. The largest suspect luminosity is seen to come from the CMX lepton type. The behaviour of this lepton type is particular in that there is a large suspect luminosity associated with the STEP1 check. This means that $R$ values obtained from different trigger paths are considerably different. $R$ fluctuations are found to highly depend on the definition of a lepton type at offline level, for a CMX muon in particular. More restrictive offline definition leads to larger differences in $R$. Conversely, the more similar the lepton type definition at offline and trigger level the smaller the fluctuations.

A particular comment regarding the period during which the performance of the COT was compromised (February-May 2004 between run $\geq 181012$ and run $\leq 181190$ ) is needed [4]. Of the total $\sim 380 \mathrm{pb}^{-1}$ of luminosity used for this study, only $5 \mathrm{pb}^{-1}$ satisfying the preselection requirements described at the end of Section B.1 were collected in this period. Furthermore, only $0.5 \mathrm{pb}^{-1}$ out of $5 \mathrm{pb}^{-1}$ are found not to be suspect for all lepton types and most of $R$ distributions for runs of this period show very different values w.r.t. each corresponding local average. For these reasons the COT compromised period has been neglected.

\section{A.5 Good run list on the basis of cross section sta- bility}

For comparison a study of the SUSY DILEPTON trigger cross section is also presented. Insofar as the cross section of a trigger path is expected to be stable in time, it may be used as monitoring variable. As in Sec. A.3 a suspect run is defined as follows. For each run and for each trigger path, two local averages of the cross section $<$ cross $>$ of the 20 runs before and after the considered run are calculated. The run is flagged as suspect if

$$
\mid \text { cross }-<\text { cross }>\mid>5 \sigma
$$

for both before/after averages, where cross is the cross section associated to the specific trigger path and $\sigma$ is the statistical error associated to cross. In Fig. A.9 A.10 A.11 A.12 cross section distribution for each trigger paths are showns and in table A.5 each trigger path is listed with the resulting suspect luminosity.

\section{A.6 Conclusions}

On the basis on the trigger monitoring procedure presented in this Appendix, the total amount of suspect run luminosity corresponds to $\sim 32 \mathrm{pb}^{-1}$ out of $\sim 380 \mathrm{pb}^{-1}$, when considering runs with one or more muon chambers marked as bad. Using in a similar way the trigger cross section as monitoring variable, the total suspect luminosity corresponds

to $\sim 90 \mathrm{pb}^{-1}$. It should be noted that the $R$-based approach not only gives explicit information about the quality of the collected events, but also it leads to a considerably larger amount of "good data". However, this study needs some more understading of the $R$ dependence on the offline single lepton definitions and the differences between $R$-based good run list and the standard CDF good run list need to be thoroughly understood. The analysis presented in this thesis relies on the good run list commonly adopted in the CDF analyses based on [3]. In this good run list all muon chambers are declared to 


\begin{tabular}{l|l}
\hline \hline TRIGGER PATH & Luminosity $\left(\mathrm{pb}^{-1}\right)$ \\
\hline CEM4_CMU4 & 8.0 \\
CEM4_CMU4_L2_CEM8_PT8_CES2_\&_TRK8 & 0.2 \\
CEM4_CMU4_L2_TRK8_L1_CMUP6_PT4 & 0.9 \\
CEM4_CMUP4 & 0.9 \\
CEM4_CMX4 & 7.0 \\
CEM4_CMX4_L2_CEM8_PT8_CES2_\&_TRK8 & 1.2 \\
CEM4_PEM8 & 21.0 \\
CEM4_PEM8_L2_CEM12_PT8 & 2.3 \\
CEM8_PEM8 & 0.9 \\
CMU4_PEM8 & 6.9 \\
CMU4_PEM8_L2_TRK8_L1_CMUP6_PT4 & 8.5 \\
CMUP4_PEM8 & 3.1 \\
CMX4_PEM8 & 2.2 \\
CEM4_CEM4 & 19.2 \\
CEM4_CEM4_L2_CEM12_PT8 & 4.9 \\
CEM4_CEM4_L2_CEM8_PT8_CES2_\&_TRK8 & 0.05 \\
CMU4_CMX4 & 5.7 \\
CMU4_CMX4_L2_TRK8_L1_CMUP6_PT4 & 1.9 \\
CMU4_CMU4 & 8.5 \\
CMU4_CMU4_L2_TRK8_L1_CMUP6_PT4 & 27.7 \\
CMUP4_CMX4 & 1.0 \\
CMUP4_CMUP4 & 24.6 \\
\hline TOTAL & 91.4 \\
\hline \hline
\end{tabular}

Table A.5: Suspect run luminosity in $p b^{-1}$ for each trigger path on the basis of cross section stability. The COT compromised period is neglected.

be in good conditions during data taking. In this case the luminosity corresponds to $\sim 320 \mathrm{pb}^{-1}$ which is roughly $12 \%$ less than the $(380-32) \mathrm{pb}^{-1}$ resulting from the use of $R$ monitoring. 

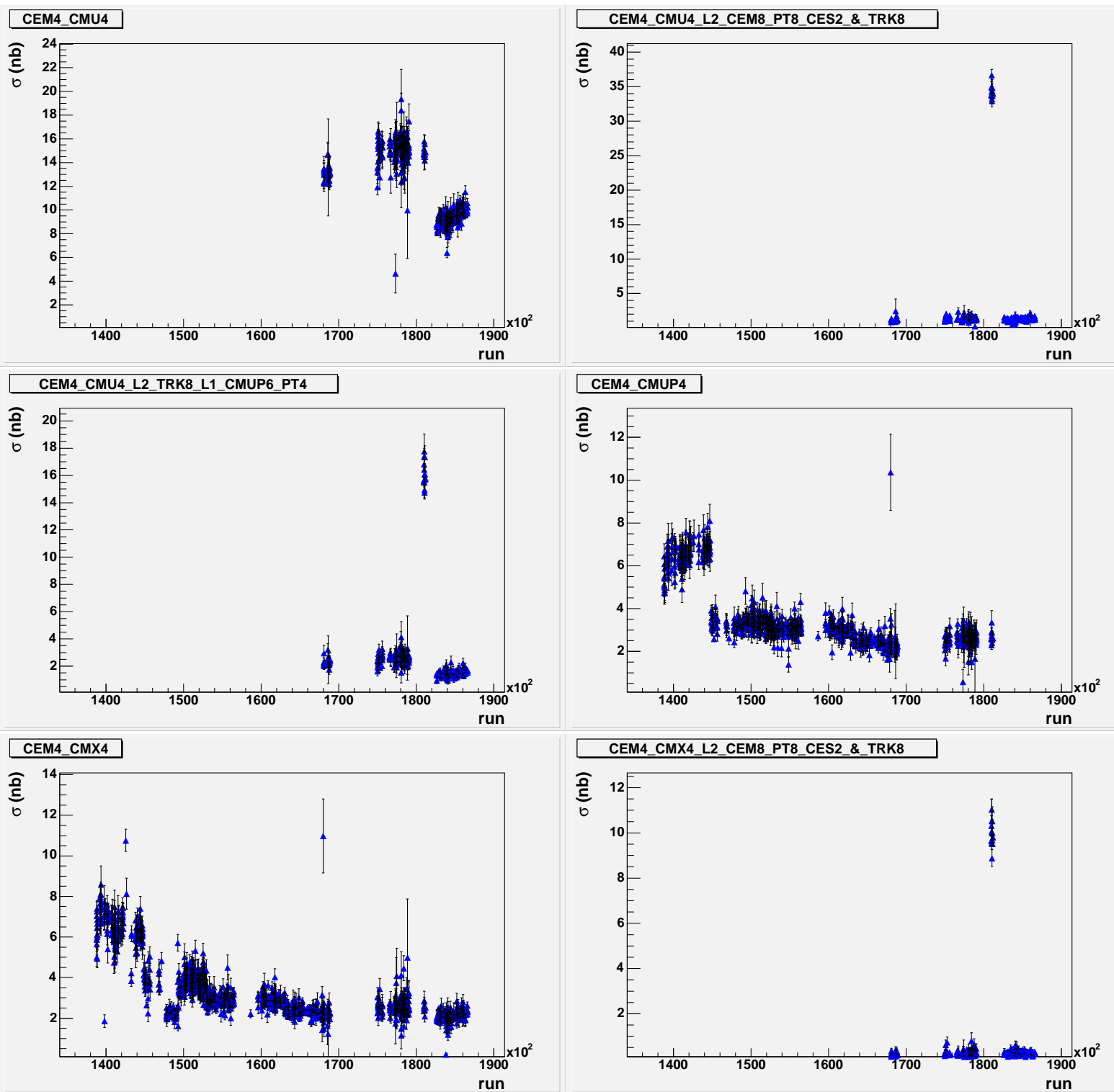

Figure A.9: Cross Section Distributions per trigger path -1-. 

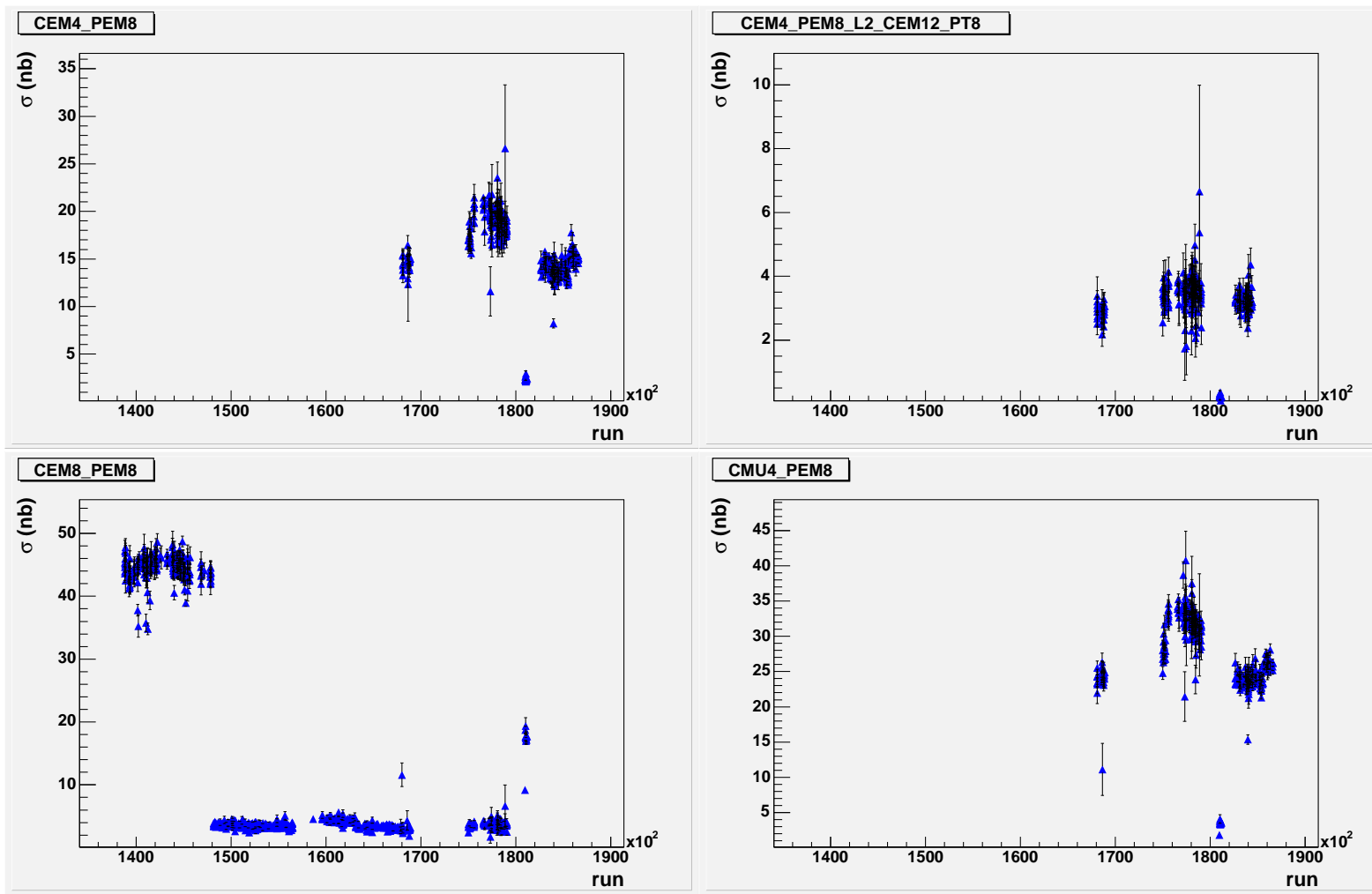

\section{CMU4 PEM8}

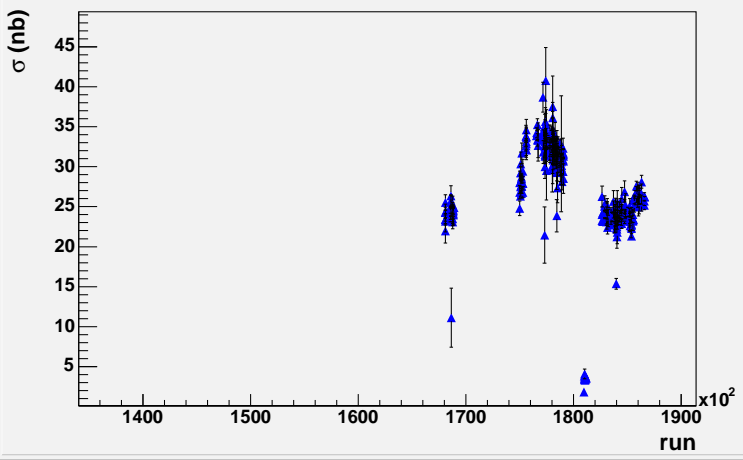

CMU4_PEM8_L2_TRK8_L1_CMUP6_PT4

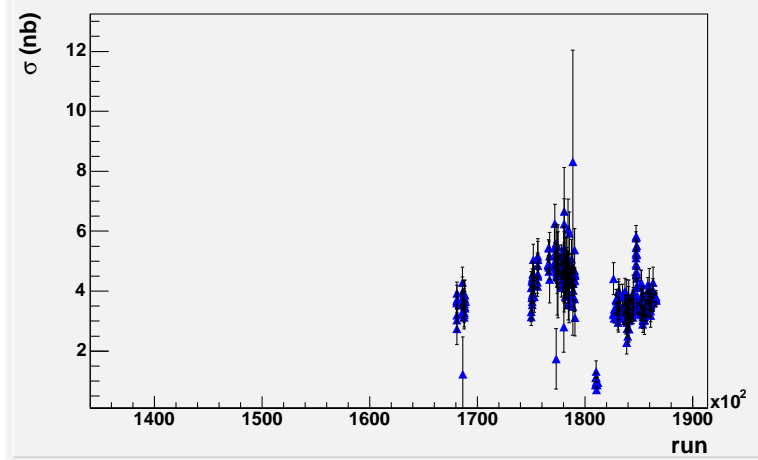

\section{CMUP4_PEM8}

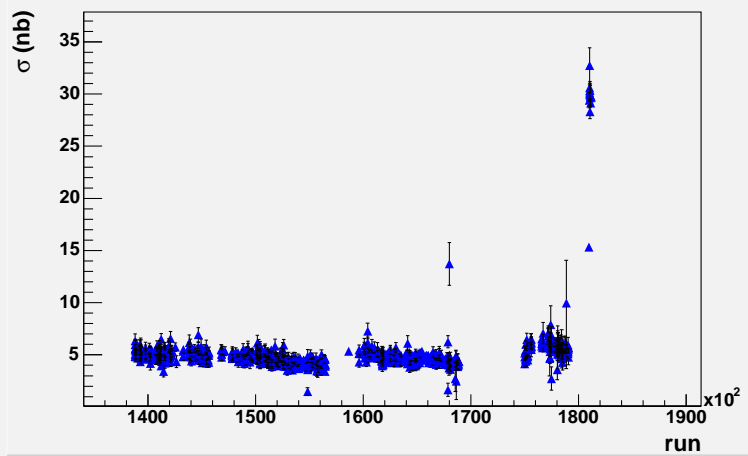

Figure A.10: Cross Section Distributions per trigger path -2-. 

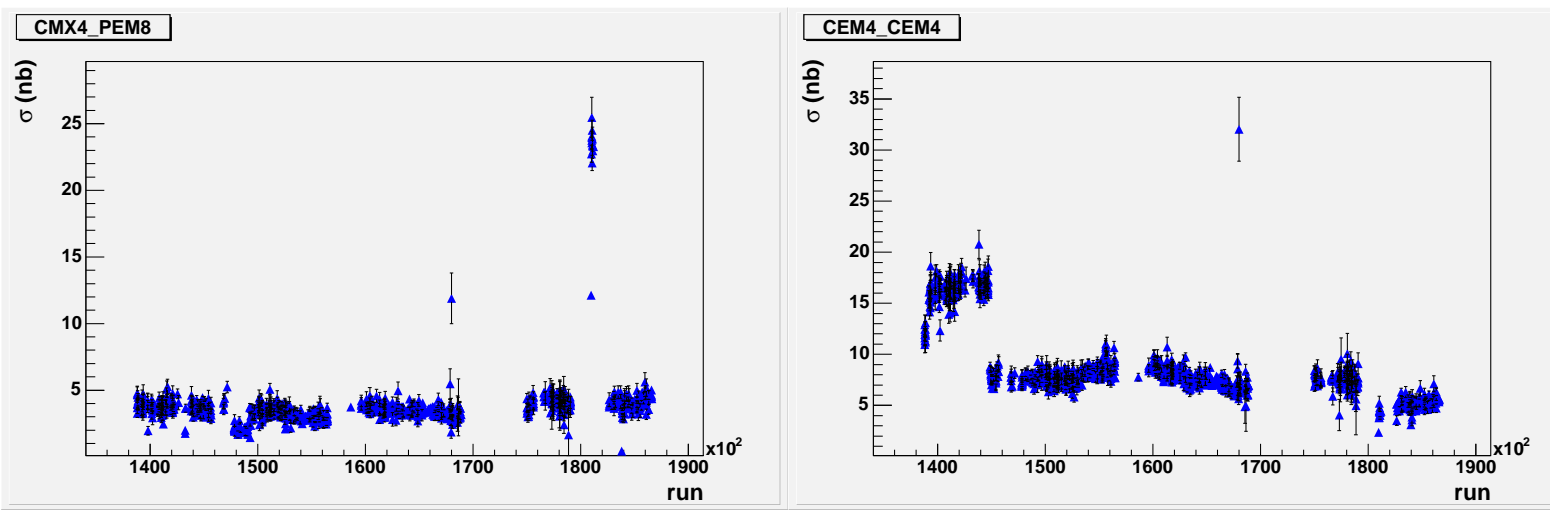

\section{CEM4_CEM4_L2_CEM12_PT8}

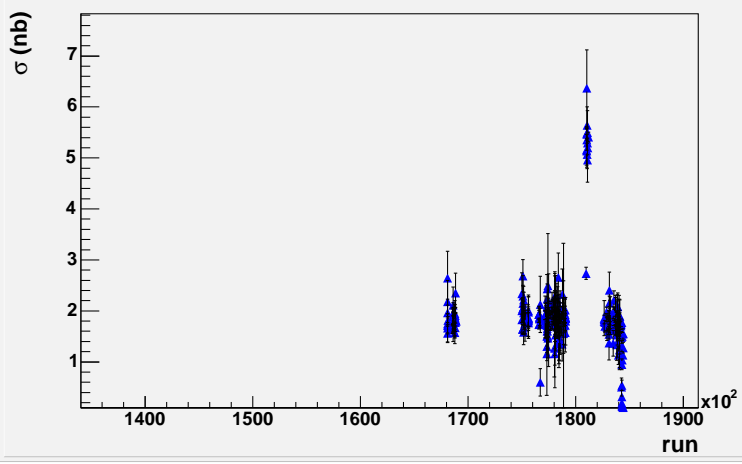

\section{CEM4_CEM4_L2_CEM8_PT8_CES2_\&_TRK8}

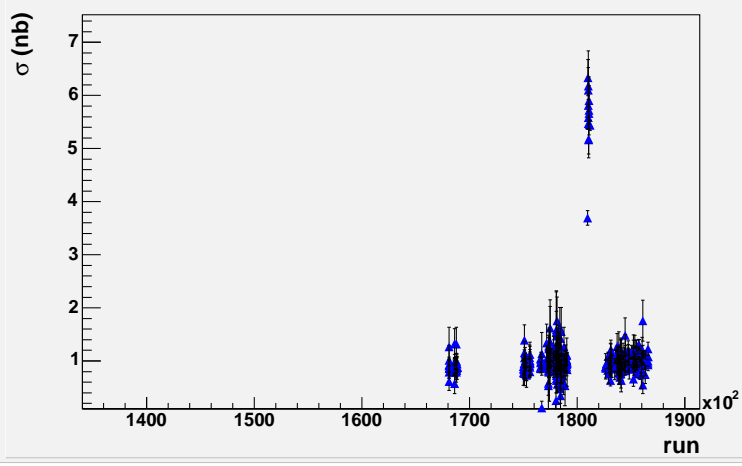

\section{CMU4_CMX4}
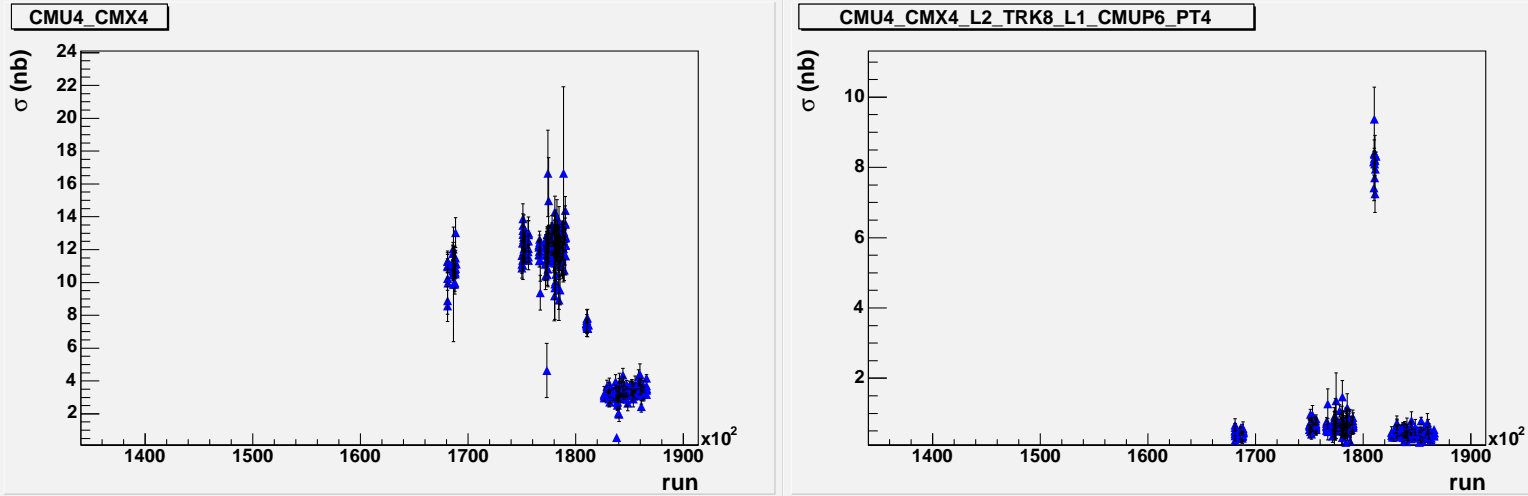

Figure A.11: Cross Section Distributions per trigger path -3-. 

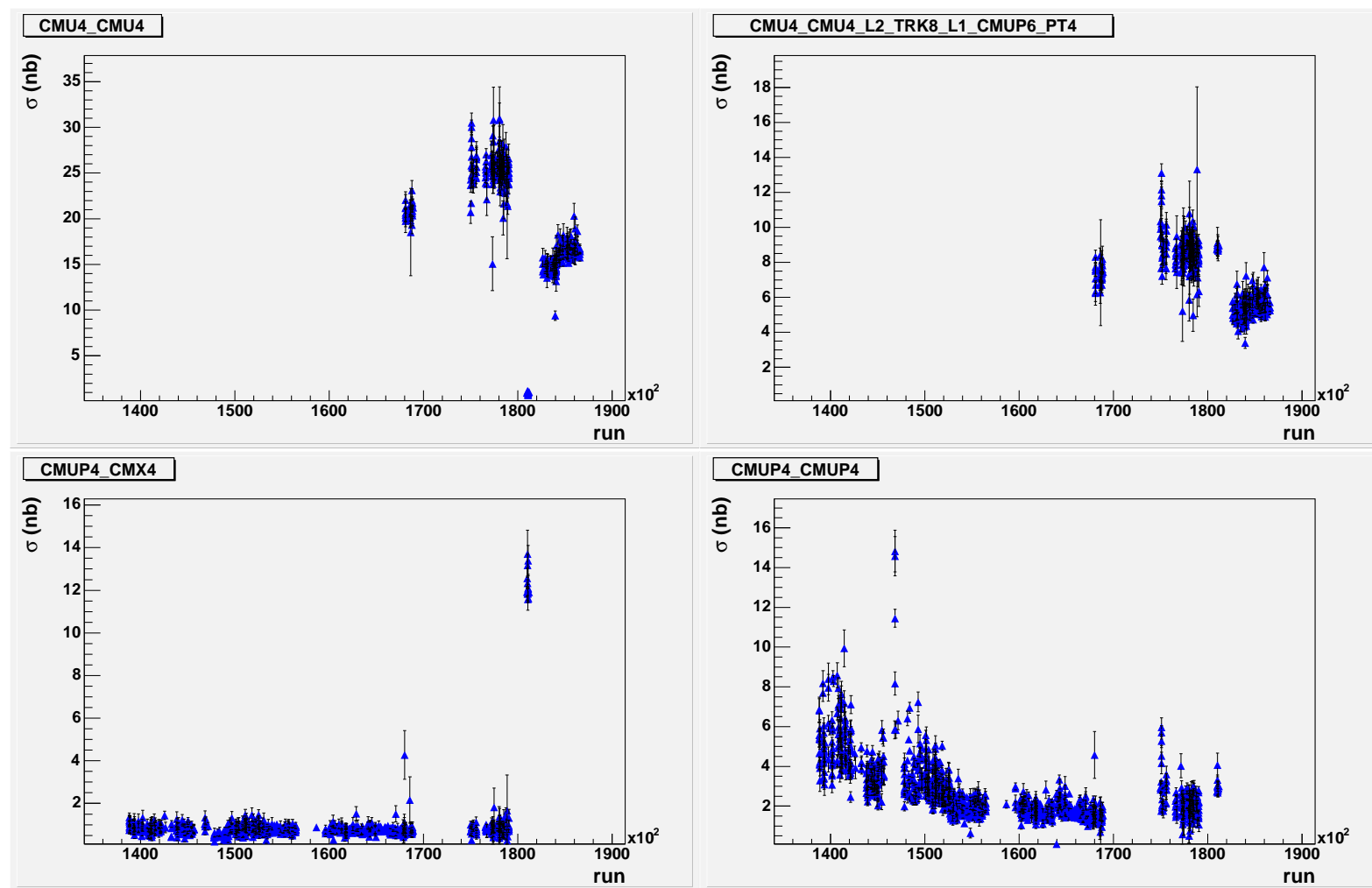

Figure A.12: Cross Section Distributions per trigger path -4-. 



\section{Bibliography}

[1] The Trigger and Datasets Working Group, CDF Run-II Trigger Table and Datasets Plan, CDF internal note 4718, Dec 2001.

[2] M.P.Giordani, S.Lammel, Dilepton Triggers for Exotic Searches at CDF II, CDF internal note 6074, Aug 2002.

[3] Data Quality Monitoring web page, http://www-cdf.fnal.gov/internal/dqm/dqm.html.

[4] For the COT compromised period refer to the web page, http://fcdfwww-cdf.fnal.gov/internal/upgrades/cot/. 



\section{Appendix B}

\section{Electron Trigger Efficiencies}

\section{B.1 Introduction}

As described in Chapter 3.2, the SUSY DILEPTON trigger involves 6 single lepton types, each of which comprises "trigger primtives" defined at specific trigger levels. In this Appendix, Level 1 and Level 2 efficiency calculations for the following single electron trigger primitives are presented:

- central electron of $4 \mathrm{GeV}$ (CEM4)

- L1_CEM4_PT4;

- L2_CEM4_PT4_CES3;

- central electron of $8 \mathrm{GeV}$ (CEM8)

- L1_CEM8_PT8;

- L2_CEM8_PT8_CES3;

- forward electron of $8 \mathrm{GeV}$ (PEM8)

- L1_EM8;

- L2_PEM8.

For this study the SUSY DILEPTON data set has been used and this has the immediate advantage that the detector conditions during data taking are naturally folded into the efficiency calculations. In order to measure the efficiency it is necessary to select a sample of candidates which can be identified as true electrons by association with a well-understood and defined production process. Unbiased conversion electrons satisfying some quality requirements serve the purpose. It is also necessary to have a simulation code which reproduces the trigger response, for this purpose an accurate simulation of the Level 1 and Level 2 trigger decision has been developed. Comparing the simulation code with the real trigger the error rate is $<1 \%$. Errors are limited to the CES related part of the trigger emulation. Considering the fact that the CES board reports a wrong information with a rate of $0.2 \%$ [2][3] and the difficulty in emulating the CES pedestal subtraction, the $1 \%$ error rate is quite acceptable. When one excludes the CES part the emulation code is in agreement with the real trigger over a sample of 20000 events (error rate is $<0.01 \%)$. 
After selecting a sufficiently clean conversion electron sample, the Level 1 efficiency is defined as the probability that an electron fulfills all Level 1 requirements. The Level 2 efficiency is defined in a similar way but with respect to the electron candidates that pass all Level 1 requirements.

\section{B.2 Sample}

The data used for this study correspond to a luminosity of around $320 \mathrm{pb}^{-1}$ (the run range considered is 138425-186598) collected from 2002 to the 2004 shutdown.In order to calculate electron efficiencies a sample which is unbiased with respect to the trigger primitive under study is needed. Two unbiased samples were extracted from the SUSY DILEPTON data set:

- the CEM unbiased sample for central electron primitives (CEM4, CEM8);

- the PEM unbiased sample for the forward electron primitive (PEM8).

The CEM unbiased sample is selected from the SUSY DILEPTON data set by requiring that the events not have been collected exclusively by a trigger path which requires a central electron at any trigger level.

The PEM unbiased sample is built up in a similar way but it also includes events collected exclusively by the CEM4_PEM8_L2_CEM12_PT8 inclusive high $p_{T}$ lepton path which does not require a forward electron primitive at Level 1 and Level 2, so that it is unbiased for our purposes. To enhance the purity of the electron candidates, they are required to fulfill all identification (ID) requirements, which are indicated in Table B.1 and B.2, for CEM and PEM respectively. Electrons must also come from a conversion, i.e. a photon converting into a electron-positron pair. The conversion is identified by means of an algorithm which associates two tracks (hereafter called the track and its sister) on the basis of geometrical conditions (see Chapter 4.4). The conversion preselection requirements are summarized in Table B.3. The track requirement limits the forward $\eta$ region under study to $|\eta|<1.5$. Electron candidates have been required to be fiducial as far as the shower max detector is concerned for both the CEM and the PEM samples. In addition to the requirements listed in the above tables, it is explicitely asked that the conversions happen inside the COT volume, i.e. that the conversion radius ( rad) satisfies $2 \mathrm{~cm} \leq \mathrm{rad} \leq 42 \mathrm{~cm}$ and that the two conversion tracks cross different trigger towers in order to avoid possible overestimation of the efficiency. After all the preselection cuts, the CEM and the PEM samples are left with 1965 and 759 events respectively. In Fig. B.1B.2-B.3 distributions for CEM and PEM conversion samples after preselection show the expected behaviour. 

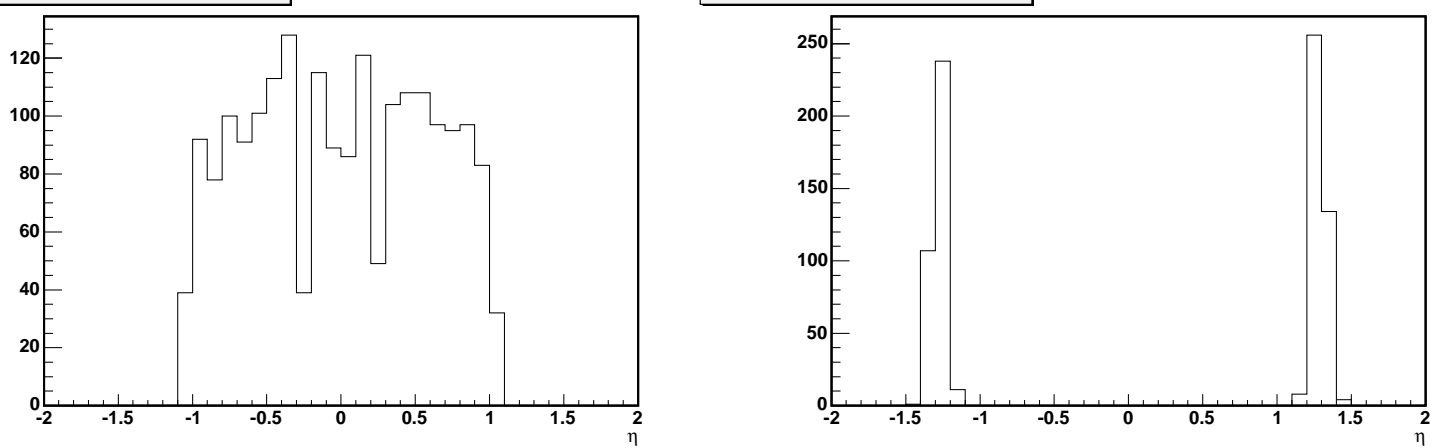

Figure B.1: $\eta$ distributions for CEM and PEM conversion candidates after ID cuts.

\begin{tabular}{c}
\hline \hline CEM ID Cuts \\
\hline \hline$E_{\text {had }} / E_{\text {em }} \leq 5 \%$ \\
$L_{\text {Shr }} \leq 0.2$ \\
COT Axial Segments (with 5 Hits) $\geq 2$ \\
COT Stereo Segments (with 5 Hits) $\geq 2$ \\
$E / p \leq 2$ \\
$-3<Q \times \Delta x<1.5 \mathrm{~cm}$ \\
$|\Delta z| \leq 5 \mathrm{~cm}$ \\
$\chi_{\text {strip }}^{2} \leq 10$ \\
$z_{\text {vertex }} \leq 60 \mathrm{~cm}$ \\
$d_{0}<0.2 \mathrm{~cm}$ \\
$E_{T}^{0.4} \leq 4 \mathrm{GeV}$ \\
\hline \hline
\end{tabular}

Table B.1: Central electron identification cuts. For variable definition see Chapter 3.

\begin{tabular}{|c|}
\hline \hline PEM ID Cuts \\
\hline \hline$E_{\text {had }} / E_{\text {em }}<5 \%$ \\
PES $5 / 9 \geq 0.65$ \\
COT Axial Segments (with 5 Hits) $\geq 2$ \\
COT Stereo Segments (with 5 Hits) $\geq 1$ \\
$E / p \leq 3$ \\
$z_{\text {vertex }} \leq 60 \mathrm{~cm}$ \\
\hline \hline
\end{tabular}

Table B.2: Forward electron identification cuts. For variable definition see Chapter 3.

\section{B.3 Efficiency Calculation and Fits}

The efficiency is defined as the probability for a real electron to pass the trigger requirements. After a sample of conversion electrons is selected, each electron candidate is matched to the corresponding online objects and it is checked if the electron passes all trigger requirements. The efficiency $\epsilon$ is interpolated using a parametrization function of one or more relevant variables (such as the calorimeter transverse energy $E_{T}$ and/or 


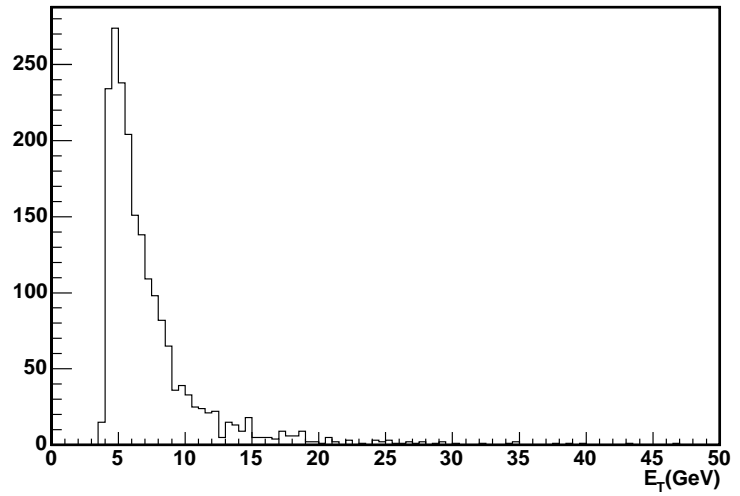

CENTRAL conversion candidates

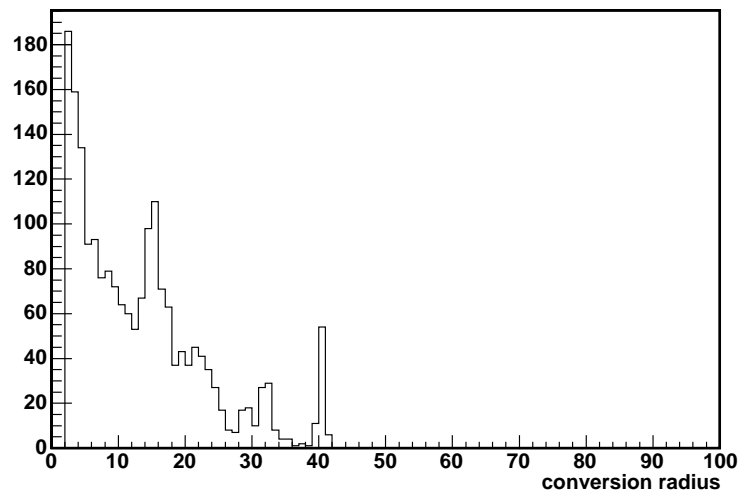

CENTRAL conversion candidates

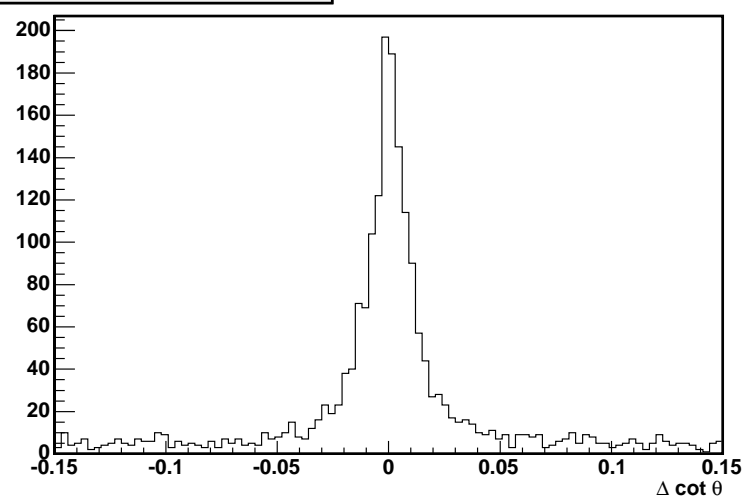

CENTRAL conversion candidates

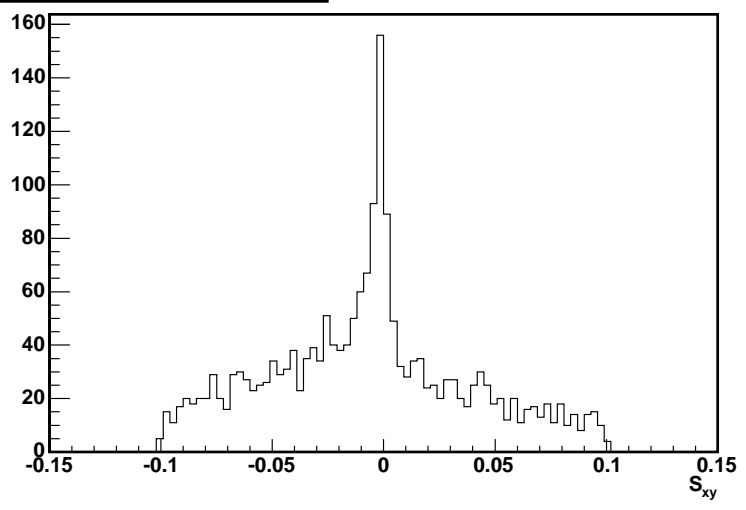

Figure B.2: Distributions for CEM conversion candidates after the whole preselction. 


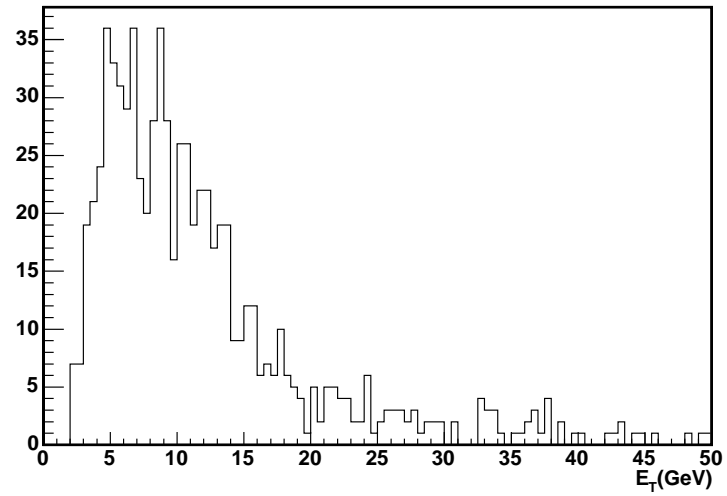

PLUG conversion candidates

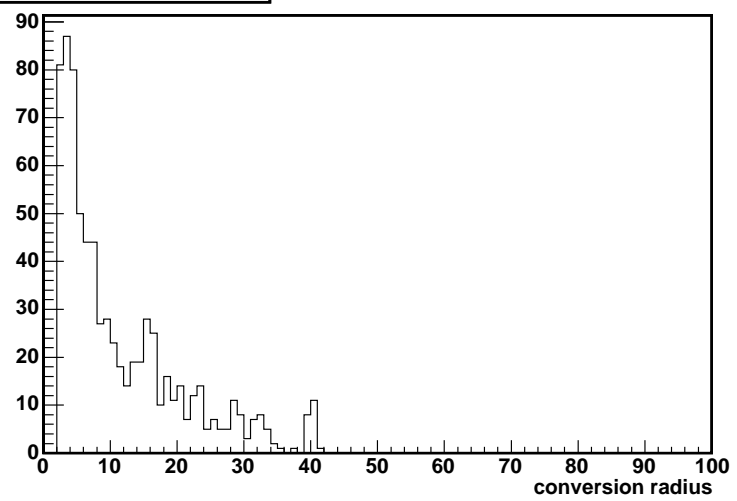

PLUG conversion candidates

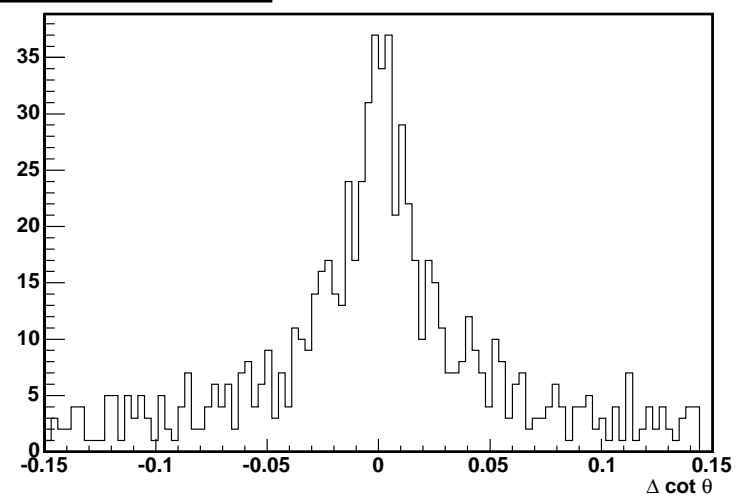

PLUG conversion candidates

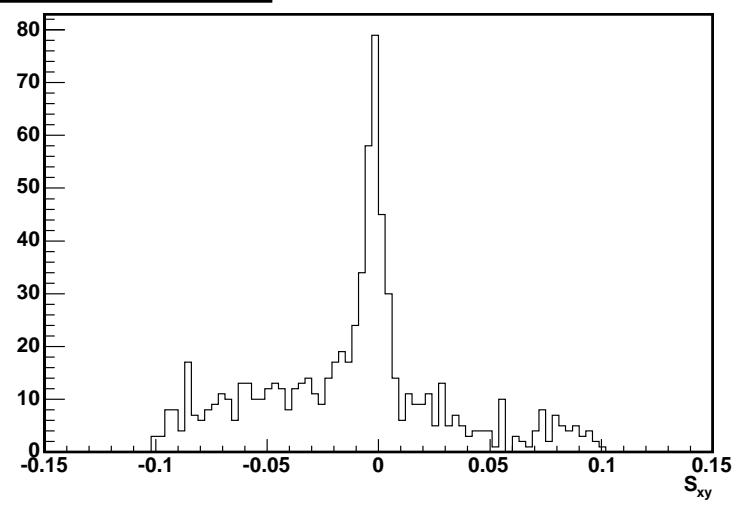

Figure B.3: Distributions for PEM conversion candidates after the whole preselection. 
$|\Delta \cot \theta|<0.15$

$\Delta S<0.1$

electron track and sister track in different trigger towers

Table B.3: Conversion preselection requirements. The variable $|\Delta \cot \theta|$ corresponds to the difference in the cotangent of the polar angle between the conversion tracks while $\Delta S$ indicates their separation in the transverse $r-\phi$ plane.

the track transverse momentrum $p_{T}$ ), in which possible correlations have to be taken into account. The parametrization function is generally defined as $\epsilon=f(\mathbf{V}, \alpha)$, where $\mathbf{V}$ is the vector of the variables chosen for the parametrization and $\alpha$ is the vector of the parameters which determine the shape of the turn-on curve. Each event has a particular value of $\mathbf{V}$. Given also some initial random values of $\alpha$, a minimazation algorithm is used to determine $\alpha$ by means of a step by step procedure. At each step of the minimazation process $\alpha$ changes till the fit converges giving as a result $\alpha_{\text {min }}$. As a consequence the resulting turn-on shape of the efficiency is $\epsilon=f\left(\mathbf{V}, \alpha_{\text {min }}\right)$. As fitting function an unbinned likelihood function, defined as

$$
L=-\prod_{i} \ln \mathrm{p}_{\mathrm{i}}
$$

is built where $p_{i}$ is the probability that a specific event passes the trigger and $\prod_{i}$ is the product of the events considered. In the efficiency calculation it is necessary to correct the result for the effect of fake electrons since real electrons and fakes could present different trigger efficiencies. In order to take this effect into account, after the conversion preselection, the remaining candidates are subdivided in two sub-samples:

- the peak region $(|\Delta \cot \theta|<0.03)$ or mixed sample;

- the sideband regions $(|\Delta \cot \theta|>0.05)$ or background sample.

In the sideband regions it is assumed that the fake contamination is $100 \%$, while in the peak region there is a mixture of real and fake electrons. Then a sideband subtraction is performed, which is basically the comparison of the two defined samples. Knowing the fraction of real $\left(n_{e}\right)$ and fake $\left(n_{\text {fake }}\right)^{1}$ electrons in the mixed sample, the efficiency for real electrons $\left(\epsilon_{e}\right)$ is related to the efficiency of fakes $\left(\epsilon_{\text {fake }}\right)$ and the efficiency of the mixed sample $\left(\epsilon_{m i x}\right)$ according to:

$$
\epsilon_{\text {mix }}=n_{e} \times \epsilon_{e}+n_{\text {fake }} \times \epsilon_{\text {fake }}
$$

Therefore, if a specific event fulfills all trigger requirements, the probability in equation B.1 can be written as $p_{i}=\epsilon_{m i x}\left(\epsilon_{\text {fake }}\right)$ if it belongs to the mixed sample (background sample). If the event does not pass the trigger $p_{i}=1-\epsilon_{\operatorname{mix}}\left(1-\epsilon_{\text {fake }}\right)$ in the mixed sample (background sample). The fake fraction is calculated, in bins of $E_{T}$, as the ratio of events in the sideband regions scaled by the corresponding interval length of $|\Delta \cot \theta|$ and events falling under the peak. The fraction of fakes as a function of transverse energy is shown in Fig. B.4 for both CEM and PEM electrons. To a first approximation, these

\footnotetext{
${ }^{1} n_{e}+n_{\text {fake }}=1$
} 
distributions have been fitted with a constant function and the fit result is given in Table B.4. After estimating the fake fraction in the mixed sample the efficiency for true electrons can be re-obtained from equation B.2:

$$
\epsilon_{e}=\frac{1}{n_{e}}\left(\epsilon_{m i x}-n_{\text {fake }} \times \epsilon_{\text {fake }}\right)
$$

In the following all the efficiency results will be corrected taking into account the effect of fakes, thus using equation B.3.

\section{CENTRAL conversion candidates}

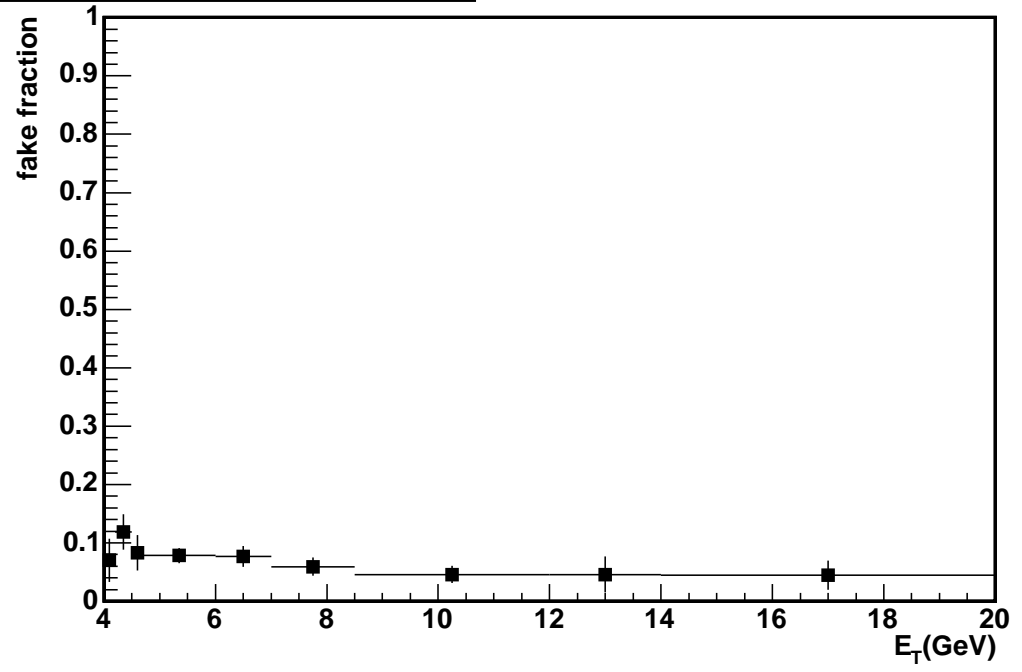

\section{PLUG conversion candidates}

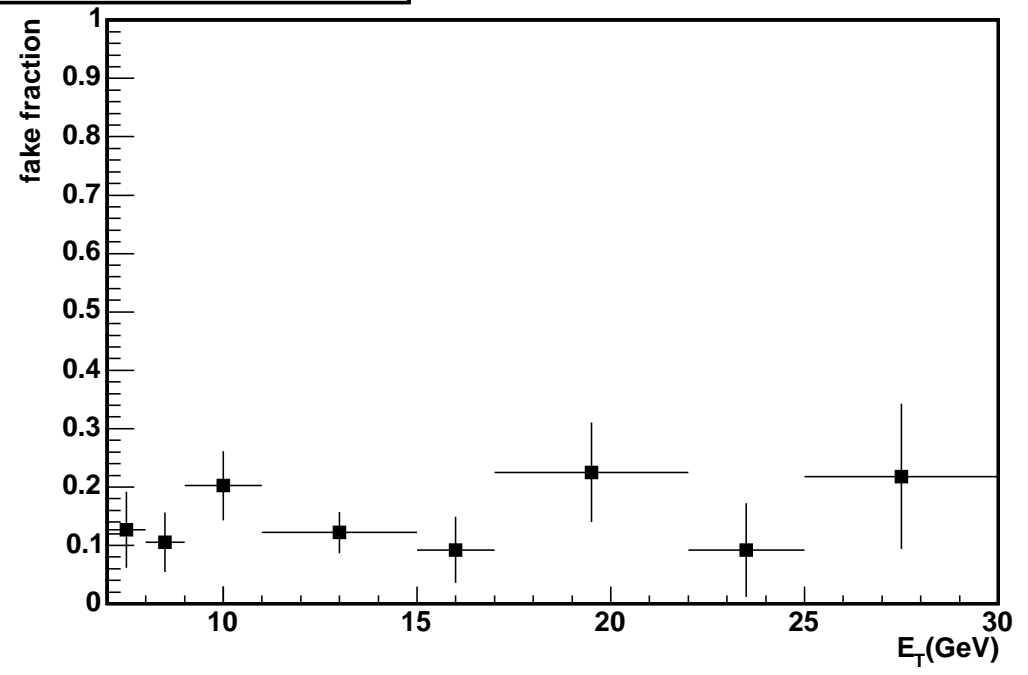

Figure B.4: PEM conversion candidates distribution after ID cuts. 


\begin{tabular}{|c|c|}
\hline \hline & Fit Result \\
CEM & $(6.6 \pm 0.7) \%$ \\
PEM & $(13 \pm 2) \%$ \\
\hline \hline
\end{tabular}

Table B.4: Estimate of the fake fraction in the CEM and PEM samples.

\section{B.4 Matching Offline Electrons to Online Objects}

Once a sufficiently clean sample of conversion candidates has been selected, the offline quantities associated with each electron have to be associated with the corresponding online quantities in order to take the decision if the electron passes the trigger or not. As far as Level 1 and Level 2 are concerned, each offline electron can be associated with:

- a trigger tower;

- an XFT track (only CEM candidates $^{2}$ );

- a Level 2 calorimeter cluster.

\section{B.4.1 Matching Offline EM Cluster to L1 Trigger Tower}

The matching between the offline electron and the Level 1 trigger tower is performed by matching the $\eta-\phi$ indices of the seed tower of the offline EM cluster to the corresponding trigger tower. Then the EM and HAD energies of the tower are used to test the trigger requirements.

\section{B.4.2 Matching Offline Track to XFT Track}

For each electron track we search for the corresponding XFT track by comparing their respective positions at each of the four axial superlayers. The offline and XFT track are considered matched at a particular superlayer if they are separated by less than 10 pixels at that superlayer. For a full match the offline and the XFT track must match at three out of four possible axial superlayers. If more XFT tracks are matched to the same offline electron, the one that fulfills all trigger requirements is chosen.

\section{B.4.3 Matching Offline EM Cluster to L2 Cluster}

The matching between the offline electron and the Level 2 cluster is performed by requiring that the matched Level 1 trigger tower be the seed tower of the Level 2 cluster. At Level 2 two types of EM clustering algorithms can be executed depending on the energy of the EM cluster:

- "high" Level 2 cluster (seed of $8 \mathrm{GeV}$, shoulder of $7.5 \mathrm{GeV}$ );

- "low" Level 2 cluster (seed of $2 \mathrm{GeV}$, shoulder $\infty$ ).

\footnotetext{
${ }^{2}$ No XFT requirements are involved in the PEM trigger primitives.
} 
For $8 \mathrm{GeV}$ trigger primitives (CEM8, PEM8) a match with a "high" Level 2 cluster is searched. For $4 \mathrm{GeV}$ trigger primitives (CEM4) a match with a "low" Level 2 cluster is required.

To demonstrate that the offline-online matching is under control in Fig.B.5 the distributions of the transverse energy of the matched Level 2 Cluster versus the corresponding offline cluster quantity has been plotted for both CEM and PEM candidates. Since only the CEM trigger primitives involve the XFT track requirements, the distribution of the transverse momentum of the XFT track versus the offline track has been plotted only for central electrons.

\section{B.5 Efficiency of Plug Electrons}

\section{B.5.1 L1_EM8 Efficiency}

In the SUSY DILEPTON trigger the efficiency of L1_EM8 trigger primitive, only used as the Level 1 requirement for the L2_PEM8 primitive, has only been studied in the forward region. In accordance with the real trigger the simulation of L1_EM8 requires a trigger tower with:

- $E_{T}^{e m} \geq 8 \mathrm{GeV}$;

- $E^{\text {had }} / E^{e m} \leq 6.25 \%$.

The efficiency of this primitive versus the offline EM transverse energy (Fig. B.6) shows a particularly slow turn-on which could be explained by the non-negligible difference between the offline EM transverse energy associated with the forward electron and the Level 1 EM transverse energy [5] (Fig. B.7). The fitting function (curve suggested in [4] for the dependence on $E_{T}$ ) used is:

$$
\epsilon=\epsilon_{\infty} \times \operatorname{freq}\left(\frac{\sqrt{E_{T}}-\sqrt{E_{T_{0}}}}{2 \sigma_{E_{T}}}\right)
$$

where $\operatorname{freq}(x)=\frac{1}{\sqrt{2 \pi}} \int_{-\infty}^{x} e^{\frac{-t^{2}}{2}} d t$, which is essencially the integral of a Gaussian function. It has been found that L1_EM8 primitive has a tighter requirement on the $E^{\text {had }} / E^{\text {em }}$ $(\sim 6.25 \%)$ in the plug region than the designed one $E^{\text {had }} / E^{e m}<12.5 \%$. To understand the impact of this requirement, the trigger simulation was run with both $E^{\text {had }} / E^{\text {em }}$ cuts: the resuling efficiencies distributions are shown in Fig. B.6. From the shape and fit results (Table B.5-B.6) it can be concluded that the change in the $E^{\text {had }} / E^{\text {em }}$ requirement does not significantly influence the efficiency calculation. 


\section{PLUG conversion candidates}

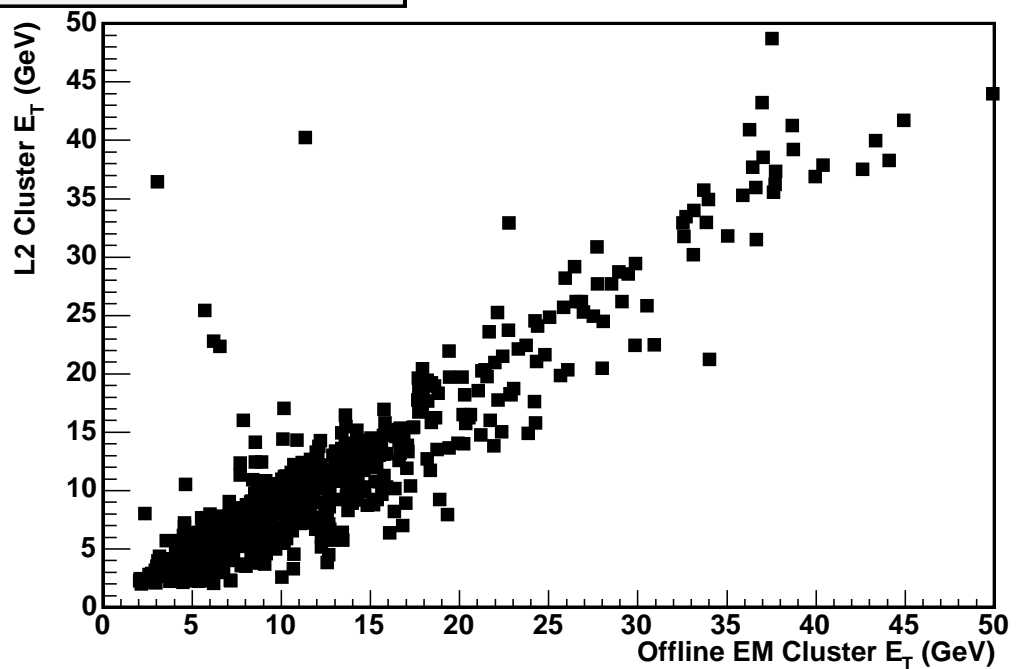

\section{CENTRAL conversion candidates}

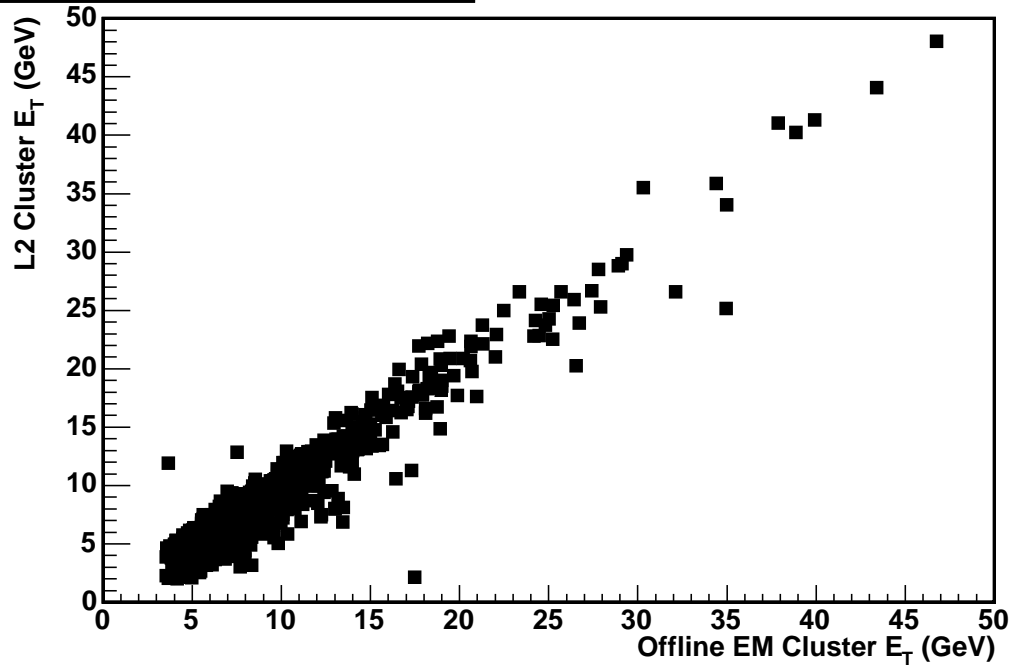

\section{CENTRAL conversion candidates}

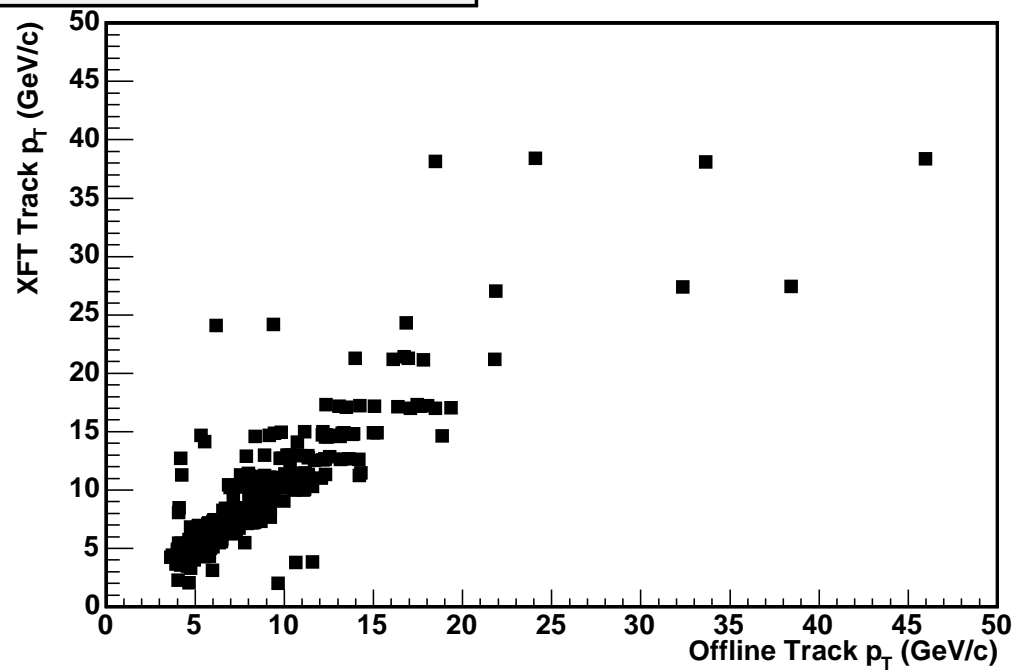

Figure B.5: Distributions of matched online quantities versus the corresponding offline ones. 

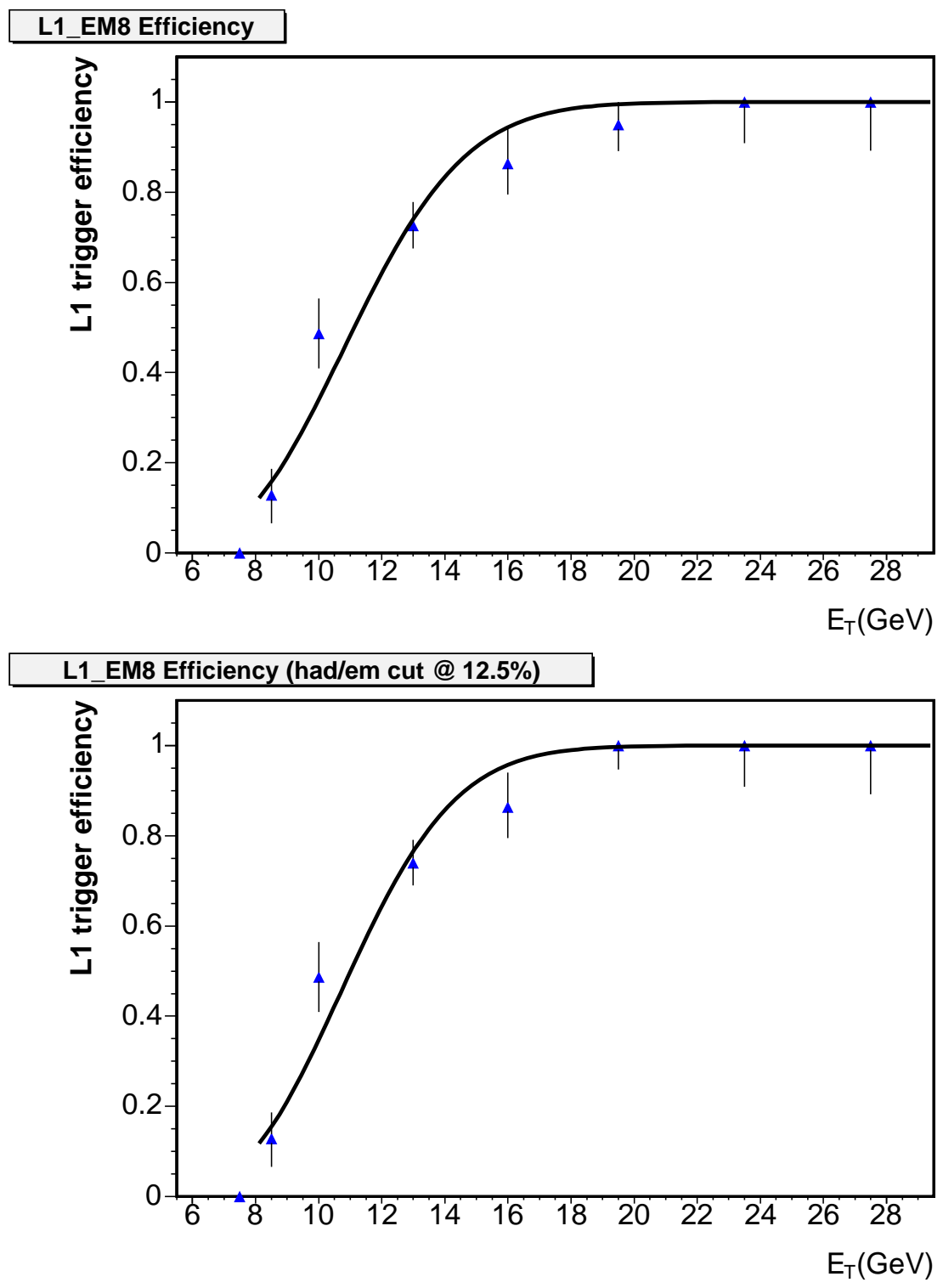

Figure B.6: Distribution of L1_EM8 efficiency for the plug electron part only as a function of the offline electron $E_{T}$. The upper plot represents the efficiency of L1_EM8 as it is currently implemented in the trigger table, while the bottom plot side shows the efficiency of L1_EM8 with the $E^{H A D} / E^{E M}$ cut at $12.5 \%$, as it should be. 


\begin{tabular}{|c|c|}
\hline \hline$\epsilon_{\infty}$ & $0.999 \pm 0.029$ \\
$E_{T_{0}}$ & $10.92 \pm 0.32$ \\
$\sigma_{E_{T}}$ & $0.199 \pm 0.026$ \\
\hline
\end{tabular}

Table B.5: Fit result for L1_EM8 efficiency as a function of $E_{T}$ as it is implemented in the trigger table.

\begin{tabular}{|c|c|}
\hline \hline$\epsilon_{\infty}$ & $1.000 \pm 0.013$ \\
$E_{T_{0}}$ & $10.83 \pm 0.30$ \\
$\sigma_{E_{T}}$ & $0.190 \pm 0.024$ \\
\hline \hline
\end{tabular}

Table B.6: Fit result for L1_EM8 efficiency as a function of $E_{T}$ with the $E^{H A D} / E^{E M}$ cut at $12.5 \%$.

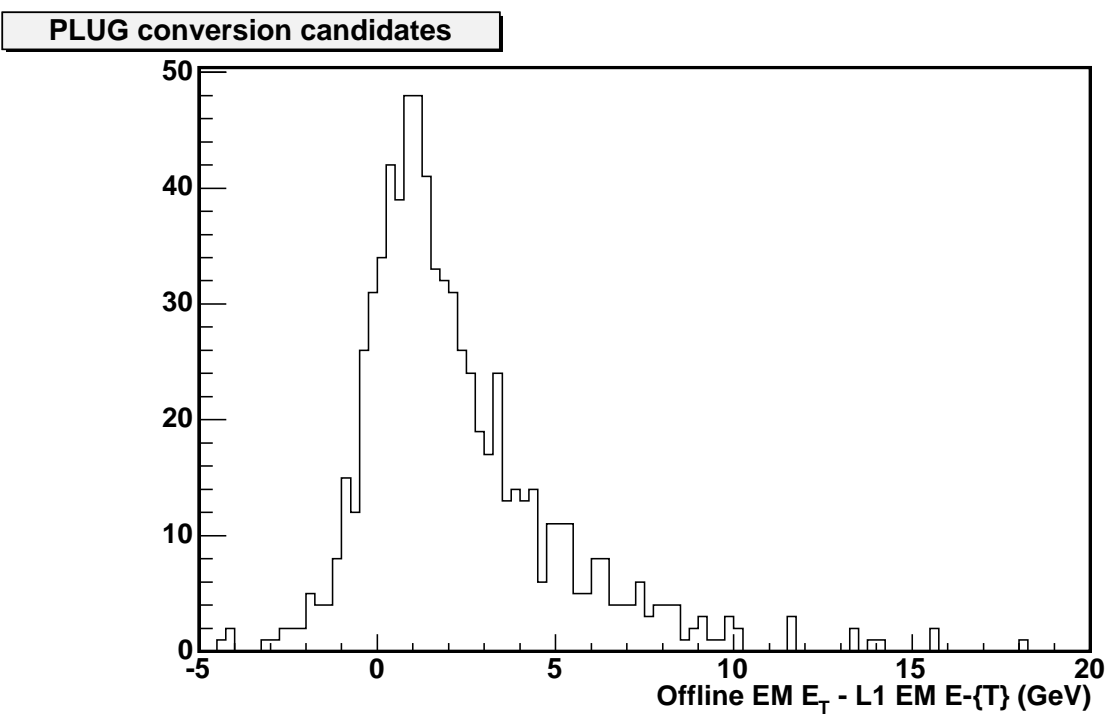

Figure B.7: Distribution of the difference between the offline EM cluster transverse energy and the corresponding L1 trigger quantity.

\section{B.5.2 L2_PEM8 Efficiency}

The L2_PEM8 trigger primitive requires a "high" L2 cluster with:

- $E_{T}^{E M} \geq 8 \mathrm{GeV}$;

- $E^{H A D} / E^{E M} \leq 12.5 \%$.

The efficiency of this primitive (Fig. B.8) has been fitted with a constant function since the distribution shows no turn-on and the fit result is shown in Table B.7. This means that once a trigger tower is found satisfying all Level 1 requirements, it is highly probable that the corresponding Level 2 cluster will satisfy all Level 2 cuts. 


\section{L2_PEM8 Efficiency}

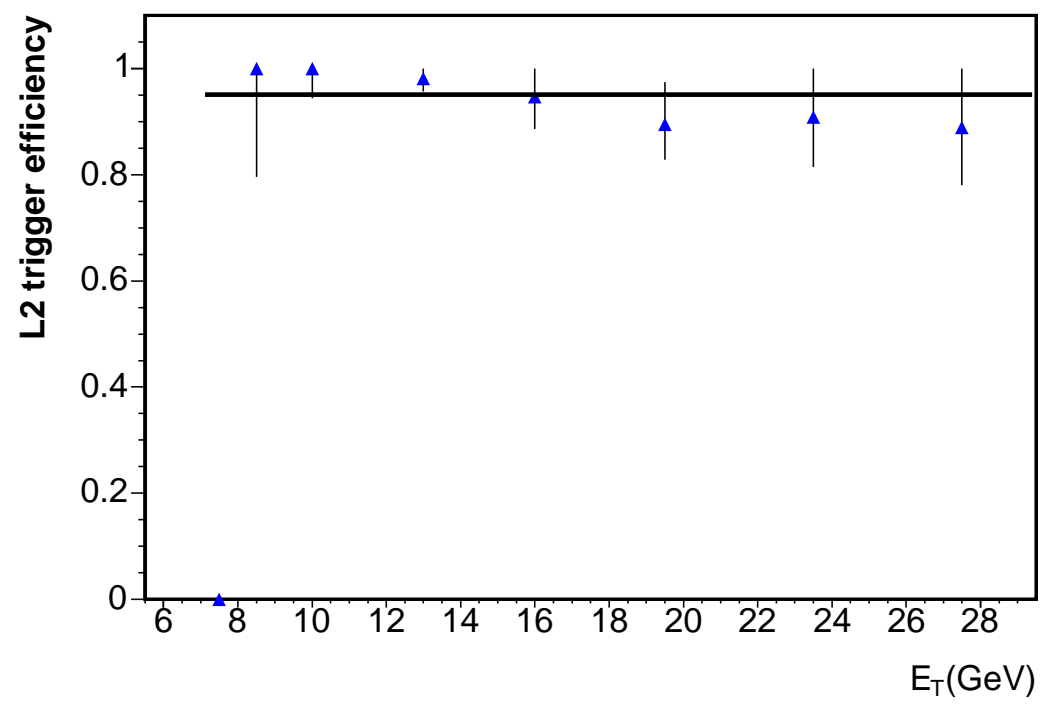

Figure B.8: Distribution of the L2_PEM8 efficiency as a function of the offline electron $E_{T}$ in $\mathrm{GeV}$ fitted with a constant.

$$
\begin{array}{|l|l|}
\hline \hline \epsilon_{\infty} & 0.943 \pm 0.013 \\
\hline \hline
\end{array}
$$

Table B.7: Fit result for L2_PEM8 efficiency as a constant function of $E_{T}$.

\section{B.6 Efficiency of Central Electrons}

\section{B.6.1 L1_CEM4_PT4 Efficiency}

The L1_CEM4_PT4 requires:

- a trigger tower with

- $E_{T}^{E M} \geq 4 \mathrm{GeV}$

$-E^{H A D} / E^{E M} \leq 12.5 \%$.

- an 4-layer XFT track with

$-p_{T} \geq 4.09 \mathrm{GeV} / \mathrm{c}$

The trigger tower and the XFT track should match in $\phi$ as the XFT tracks have only $\phi$ information. In order to disentangle the contributions coming from the calorimeter and XFT trigger requirements, two 1-dimensional fits are performed:

- the efficiency as a function of the electron calorimeter $E_{T}$, when no XFT requirements are applied (Fig. B.9);

- the efficiency of the XFT related portion of the trigger as a function of track $p_{T}$ when no calorimeter requirements are applied (Fig. B.10). 
The function used to fit the efficiency as a function of the $E_{T}$ is:

$$
\epsilon\left(E_{T}\right)=\epsilon_{\infty} \times \operatorname{freq}\left(\frac{\sqrt{E_{T}}-\sqrt{E_{T_{0}}}}{2 \sigma_{E_{T}}}\right)
$$

While for fitting the efficiency as a function of $p_{T}$ a constant function has been chosen (since the distribution does not show any clear evidence of turn-on). Fit results are shown in Table B.8-B.9.

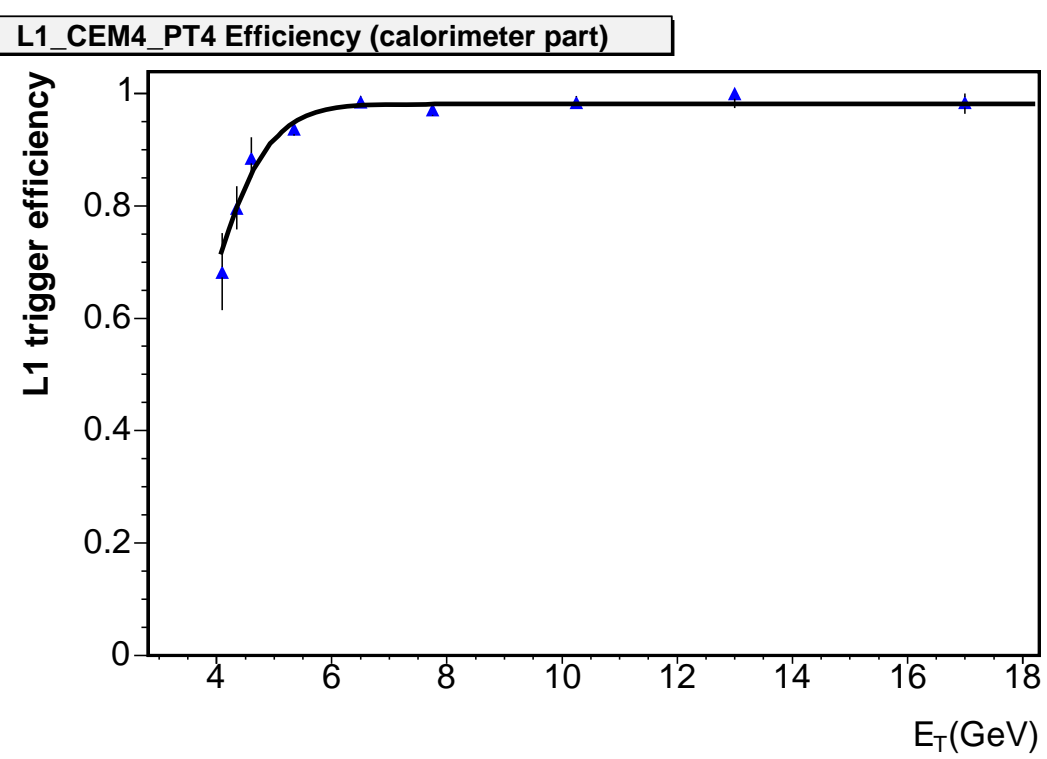

Figure B.9: Distribution of L1_CEM4_PT4 efficiency as a function of the offline electron $E_{T}$.

\begin{tabular}{|c|c|}
\hline \hline$\epsilon_{\infty}$ & $0.985 \pm 0.005$ \\
$E_{T_{0}}$ & $3.45 \pm 0.23$ \\
$\sigma_{E_{T}}$ & $0.120 \pm 0.027$ \\
\hline \hline
\end{tabular}

Table B.8: Fit result for L1_CEM4_PT4 efficiency as a function of $E_{T}$.

$$
\begin{array}{|l|l|}
\hline \hline \epsilon_{\infty} & 0.933 \pm 0.007 \\
\hline \hline
\end{array}
$$

Table B.9: Fit result for L1_CEM4_PT4 efficiency as a constant function of $p_{T}$. 


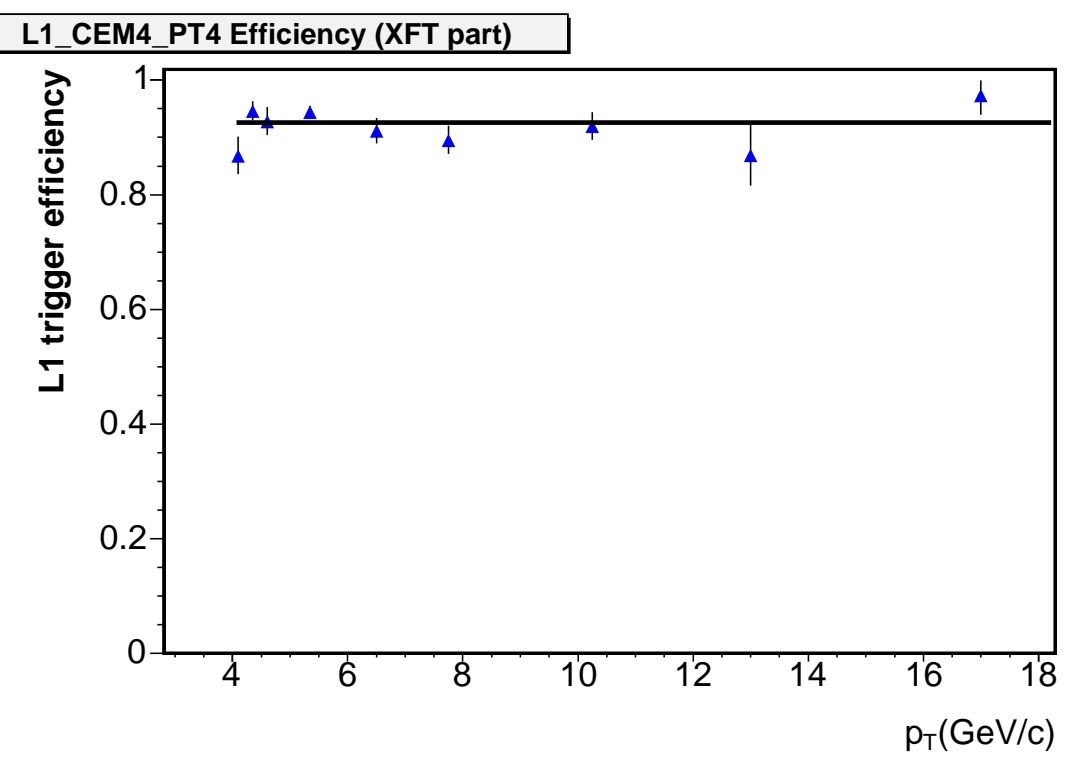

Figure B.10: Distribution of L1_CEM4_PT4 efficiency as a function of the offline electron $p_{T}$.

\section{B.6.2 L1_CEM8_PT8 Efficiency}

The L1_CEM8_PT8 is requires:

- a trigger tower with

- $E_{T}^{E M} \geq 8 \mathrm{GeV}$

$-E^{H A D} / E^{E M} \leq 12.5 \%$.

- an XFT track with

$$
-p_{T} \geq 8.34 \mathrm{GeV} / \mathrm{c}
$$

This trigger primitive has been treated similarly to L1_CEM4_PT4 and the same fitting function has been used to parametrize the efficiency as a function of $E_{T}$ (Fig. B.11). To describe the $p_{T}$ dependence of the efficiency, a function that describes the distribution well is (in Fig. B.12):

$$
\epsilon\left(p_{T}\right)=\epsilon_{\infty} \times f r e q\left(\frac{p_{T}-p_{T_{0}}}{2 \sigma_{p_{T}} p_{T} p_{T_{0}}}\right)
$$

This function differs from Eq. B.5 because it accounts for the fact that, whereas the $E_{T}$ resolution depends on $\sqrt{E_{T}}$, the $p_{T}$ resolution goes as $\frac{1}{p_{T}}([4])$. The fits are shown in Fig. B.11-B.12 and the corresponding parameter values are in Tab. B.10-B.11. 


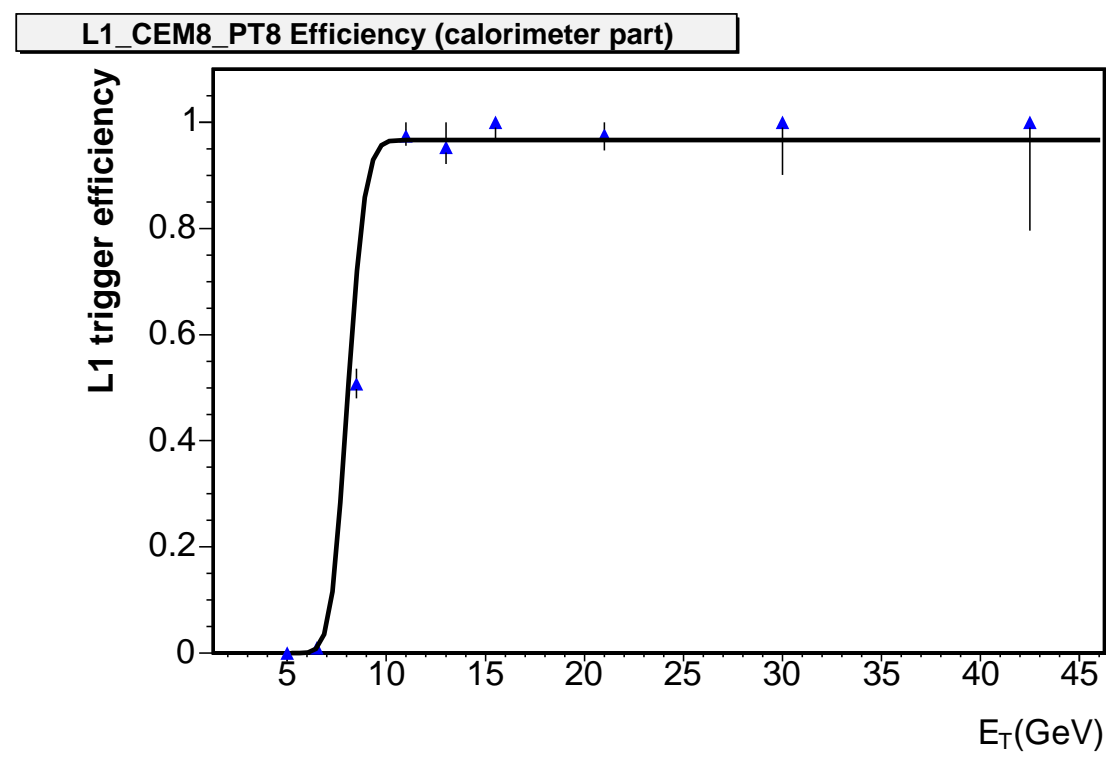

Figure B.11: Distribution of L1_CEM8_PT8 efficiency as a function of the offline electron $E_{T}$.

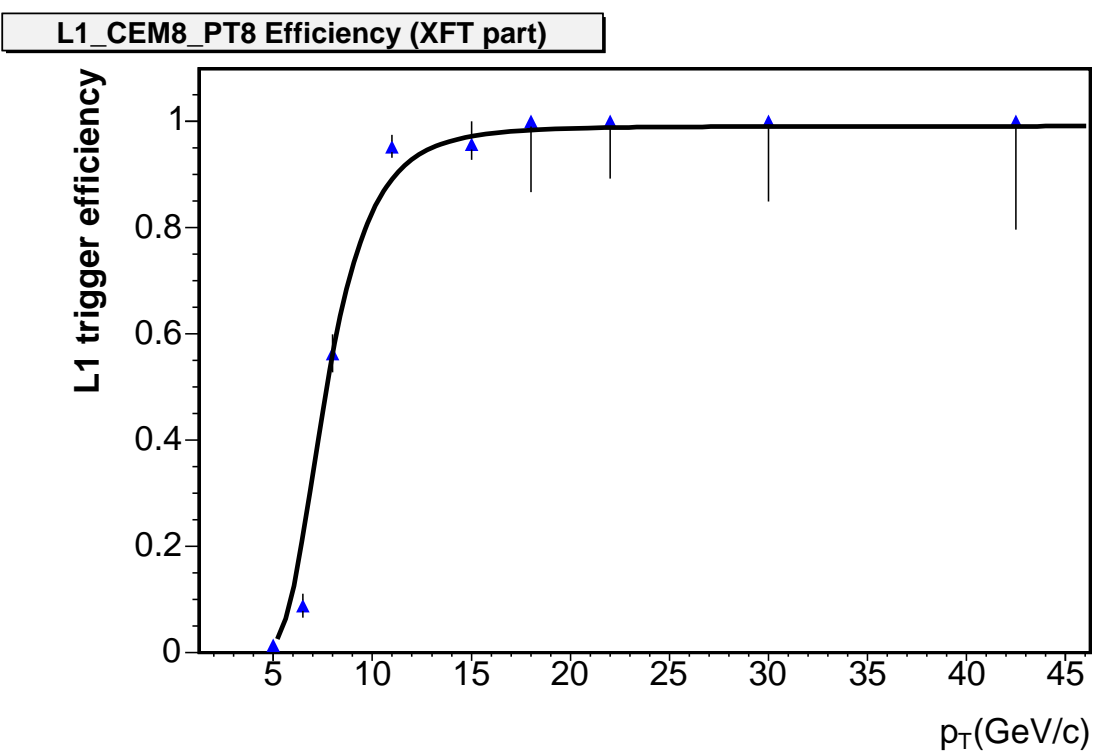

Figure B.12: Distribution of L1_CEM8_PT8 efficiency as a function of the offline electron $p_{T}$. 


\begin{tabular}{|c|c|}
\hline \hline$\epsilon_{\infty}$ & $0.97 \pm 0.01$ \\
$E_{T_{0}}$ & $8.03 \pm 0.07$ \\
$\sigma_{E_{T}}$ & $0.058 \pm 0.007$ \\
\hline \hline
\end{tabular}

Table B.10: Fit result for L1_CEM8_PT8 efficiency as a function of $E_{T}$.

\begin{tabular}{|c|c|}
\hline \hline$\epsilon_{\infty}$ & $0.992 \pm 0.009$ \\
$p_{T_{0}}$ & $7.66 \pm 0.12$ \\
$\sigma_{p_{T}}$ & $0.0151 \pm 0.0009$ \\
\hline \hline
\end{tabular}

Table B.11: Fit result for L1_CEM8_PT8 efficiency as a function of $p_{T}$.

\section{B.6.3 2-Dimensional Fit for L1 Central Electron Primitives}

The L1_CEM4_PT4 and the L1_CEM8_PT8 trigger primitives depend on both calorimetric and XFT requirements. In Section B.6.1 and B.6.2 the efficiencies of these two primitives have been studied separately as a function of $E_{T}$ and of $p_{T}$ so as to disentangle the calorimetric and the XFT dependence. To take the correlations between the transverse energy and the transverse momentum into account a 2-dimensional fit has been perfomed using the following function ([4]):

$$
\epsilon\left(E_{T}, p_{T}\right)=\epsilon_{\infty} \times \operatorname{freq}\left(\frac{\sqrt{E_{T}}-\sqrt{E_{T_{0}}}}{2 \sigma_{E_{T}}}\right) \times \operatorname{freq}\left(\frac{p_{T}-p_{T_{0}}}{2 \sigma_{p_{T}} p_{T} p_{T_{0}}}\right)
$$

which is the convolution of the Eq. B.5 and Eq. B.6.

\begin{tabular}{|c|c|}
\hline \hline$E_{T_{0}}$ & $3.84441 \pm 0.00007$ \\
$\sigma_{E_{T}}$ & $0.063428 \pm 0.000009$ \\
$p_{T_{0}}$ & $3.377 \pm 5.641$ \\
$\sigma_{p_{T}}$ & $0.000462 \pm 0.007836$ \\
$\epsilon_{\infty}$ & $0.89998 \pm 0.00001$ \\
\hline \hline
\end{tabular}

Table B.12: 2-Dimensional fit result for L1_CEM4_PT4.

\begin{tabular}{|c|c|}
\hline \hline$E_{T_{0}}$ & $7.788 \pm 0.189$ \\
$\sigma_{E_{T}}$ & $0.059 \pm 0.024$ \\
$p_{T_{0}}$ & $7.79 \pm 0.14$ \\
$\sigma_{p_{T}}$ & $0.0067 \pm 0.0013$ \\
$\epsilon_{\infty}$ & $0.964 \pm 0.017$ \\
\hline \hline
\end{tabular}

Table B.13: 2-Dimensional fit result for L1_CEM8_PT8. 


\section{B.6.4 L2_CEM4_PT4_CES3 Efficiency}

The L2_CEM4_PT4_CES3 is requires:

- a "low" Level 2 Cluster with

$$
\begin{aligned}
& -E_{T}^{E M} \geq 4 \mathrm{GeV} \\
& -E^{H A D} / E^{E M} \leq 12.5 \% .
\end{aligned}
$$

- an 4-layer XFT track with

$$
\text { - } p_{T} \geq 4.09 \mathrm{GeV} / \mathrm{c}
$$

- a CES 4-wire sum with

$$
\text { - } E_{T} \geq 3 \mathrm{GeV} / \mathrm{c}
$$

The Level 2 differs from Level 1 in the Level 2 clustering, the energy granularity and the CES requirement In Spring 2004 the high CES threshold was raised from 2 to $3 \mathrm{GeV}$.

Distributions for both L2_CEM4_PT4_CES3 and L2_CEM4_PT4_CES2 are presented in Fig. B.13 for comparison. These plots shows that the raise from 2 to $3 \mathrm{GeV}$ in the high CES wire threshold contributes to slow down the turn-on of the distribution. The main difference between L1 and L2 is the addition of the CES requirement, therefore only the $E_{T}$ dependence has been studied. The fitting function used for this trigger primitive is:

$$
\epsilon\left(E_{T}\right)=\epsilon_{\infty}\left(1-\exp \left(-\frac{E_{T}-E_{T_{0}}}{2 \sigma_{E_{T}}}\right)\right.
$$

and the corresponding fit results are shown in Fig. B.13 and Table B.14 and Table B.15.

$$
\begin{array}{|c|c|}
\hline \hline \epsilon_{\infty} & 9.93845 \mathrm{e}-01 \pm 4.62372 \mathrm{e}-03 \\
E_{T_{0}} & 9.99995 \mathrm{e}-01 \pm 8.99782 \mathrm{e}-01 \\
\sigma_{E_{T}} & 7.15896 \mathrm{e}-01 \pm 5.06622 \mathrm{e}-02 \\
\hline \hline
\end{array}
$$

Table B.14: Fit result for L2_CEM4_PT4_CES2.

$$
\begin{array}{|c|c|}
\hline \hline \epsilon_{\infty} & 9.99996 \mathrm{e}-01 \pm 3.05238 \mathrm{e}-02 \\
E_{T_{0}} & 1.00026 \mathrm{e}-01 \pm 8.08115 \mathrm{e}-01 \\
\sigma_{E_{T}} & 1.41905 \mathrm{e}+00 \pm 5.92956 \mathrm{e}-02 \\
\hline \hline
\end{array}
$$

Table B.15: Fit result for L2_CEM4_PT4_CES3. 


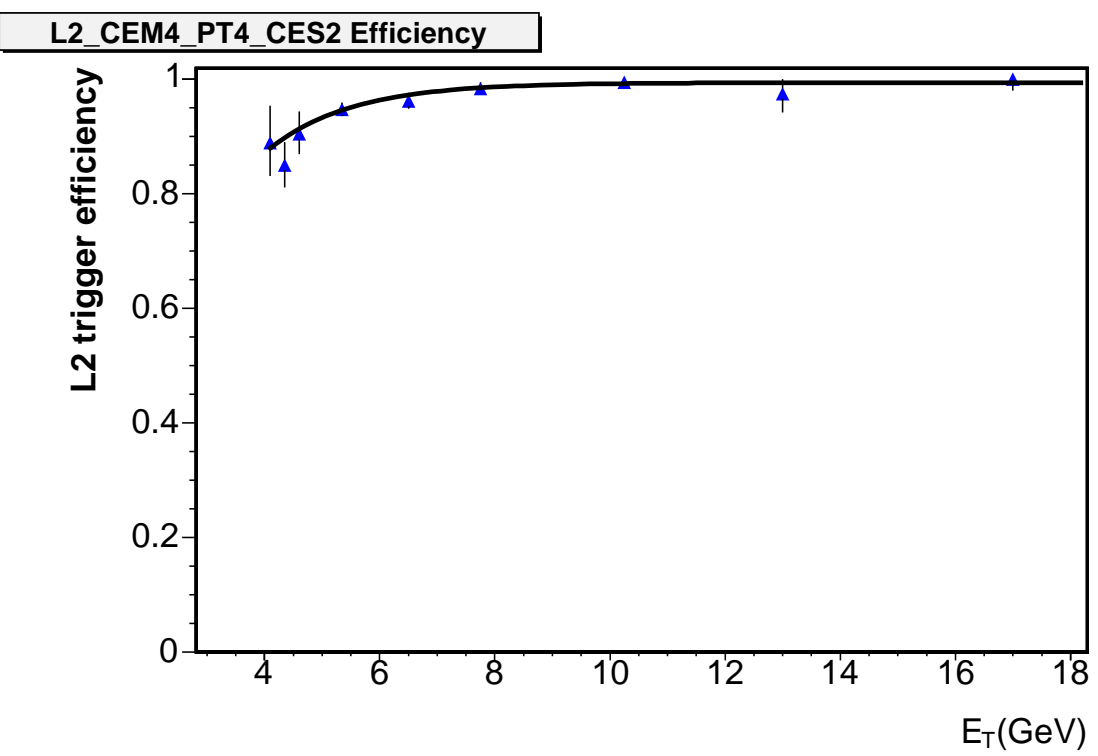

\section{L2_CEM4_PT4_CES3 Efficiency}

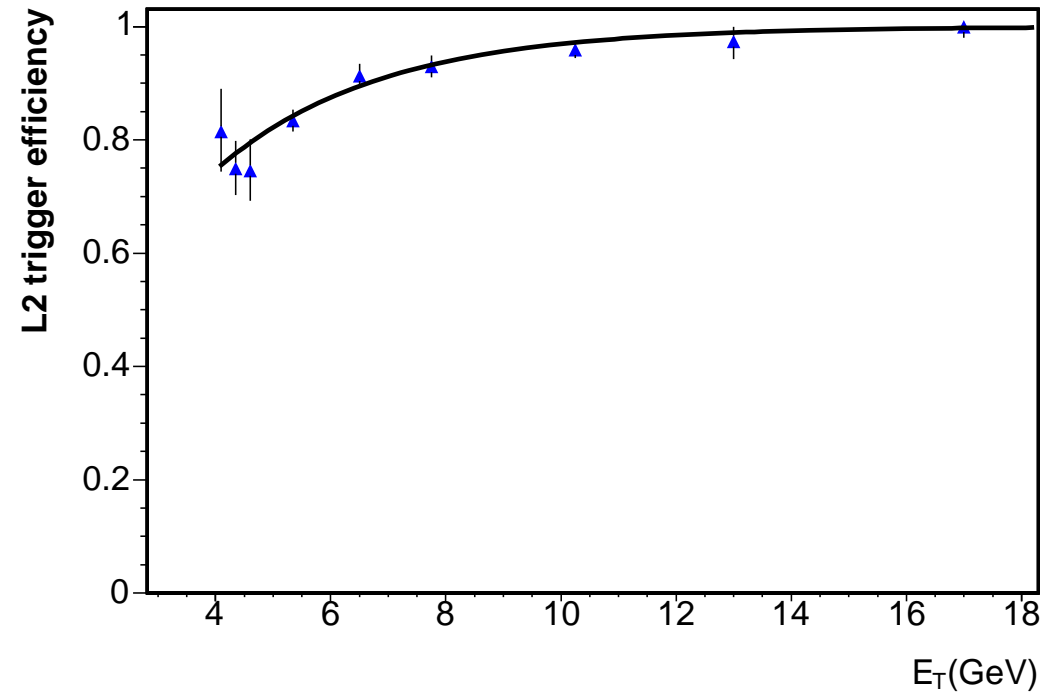

Figure B.13: Distribution of the L2_CEM4_PT4_CES2 and L2_CEM4_PT4_CES3 as a function of $E_{T}$. 


\section{B.6.5 L2_CEM8_PT8_CES3 Efficiency}

The L2_CEM8_PT8_CES3 is requires:

- a "high" Level 2 Cluster with

$$
\begin{aligned}
& -E_{T}^{E M} \geq 8 \mathrm{GeV} ; \\
& -E^{H A D} / E^{E M} \leq 12.5 \% .
\end{aligned}
$$

- an XFT track with

$$
\text { - } p_{T} \geq 8.34 \mathrm{GeV} / \mathrm{c}
$$

- a CES 4-wire sum with

$$
\text { - } E_{T} \geq 3 \mathrm{GeV} / \mathrm{c}
$$

In Fig. B.14 the efficiency distributions as a function of $E_{T}$ are presented with the $2 \mathrm{GeV}$ and the $3 \mathrm{GeV}$ CES wire thresholds. The raise in threshold did not affect significantly the efficiency as it was for the L2_CEM4_PT4_CES3 case. These efficiency distributions show no clear turn-on then they have been fitted with a constant function and the result of the fit is shown in Table B.16 and Table B.17.

$$
\begin{array}{|l|l|}
\hline \hline \epsilon_{\infty} & 9.46662 \mathrm{e}-01 \pm 1.48458 \mathrm{e}-02 \\
\hline \hline
\end{array}
$$

Table B.16: Fit result for L2_CEM8_PT8_CES2.

\begin{tabular}{|l|l|}
\hline \hline$\epsilon_{\infty}$ & $9.42643 \mathrm{e}-01 \pm 1.53231 \mathrm{e}-02$ \\
\hline \hline
\end{tabular}

Table B.17: Fit result for L2_CEM8_PT8_CES3.

\section{B.7 Systematics}

The systematic uncertainties have been calculated with respect to three kind of possible effects:

- the fake fraction used in the sideband subtraction,

- the isolation requirement of the electron,

- the requirement on the impact parameter $d_{0}$ of the track associated with the electron candidate. 

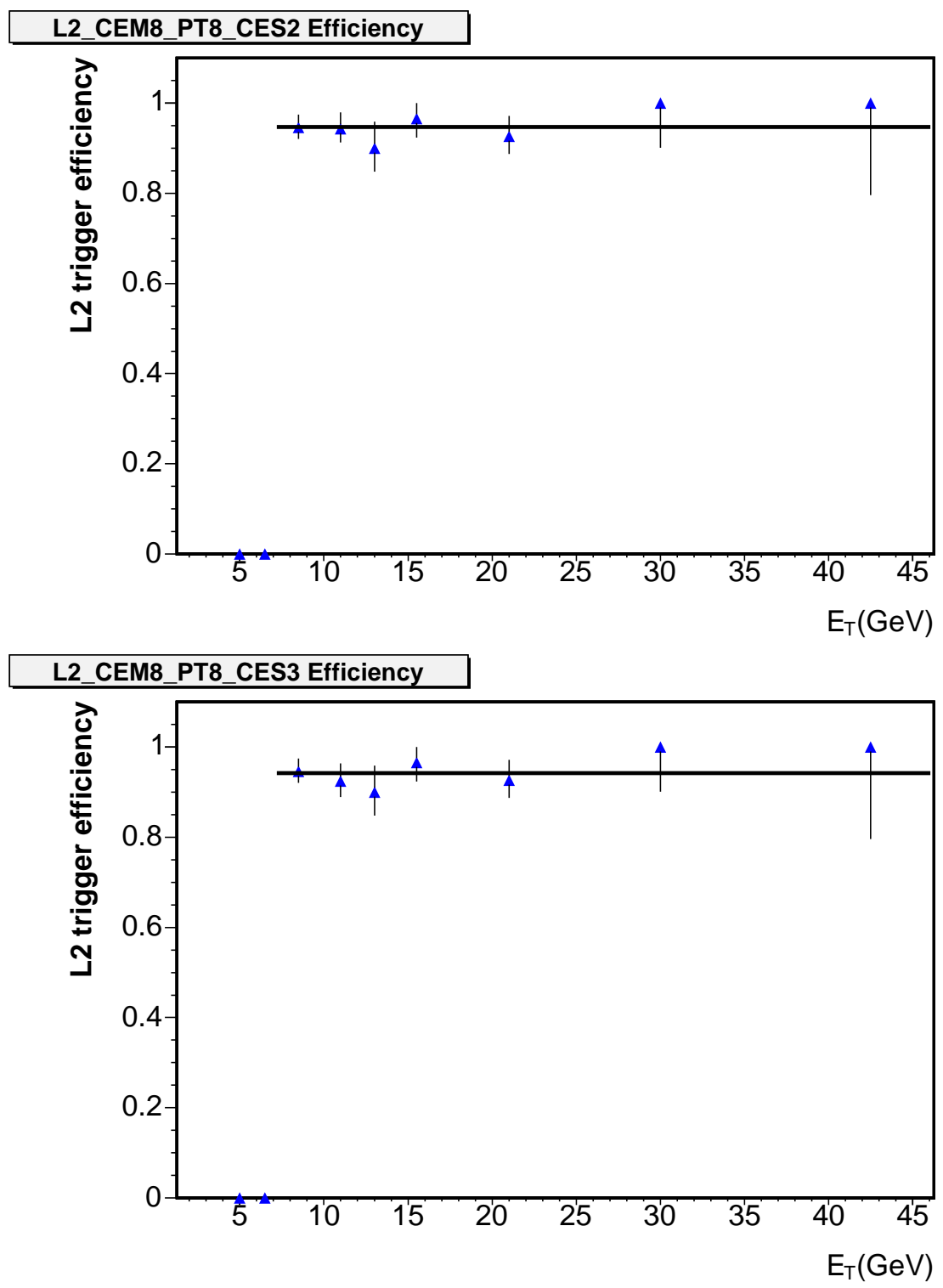

Figure B.14: Distribution of the L2_CEM8_PT8_CES2 and L2_CEM8_PT8_CES3 as a function of $E_{T}$. 
To a first degree of approximation we are considering the fake fraction to be constant as a function of the transverse energy because the corresponding distributions (see Fig.B.4) could be reasonably be fitted with a constant function. In order to consider possible variations of the fake fraction in different $E_{T}$ bins, this effect has been taken into account as a systematic uncertainty.

As far as the isolation requirement is concerned, possible large backgrounds for conversions like non-converting photon pairs from $\pi_{0}$ decay have to be accounted for. For each electron, neighboring trigger towers may acquire some additional energy. If these towers are included in the same Level 2 cluster this could shift the had/em ratio outside the allowed range. This fact may result in a possible dependence of the efficiency calculation on the isolation of the electrons. Therefore a systematic associated with the isolation has been calculated both for the CEM and for the PEM samples.

The last effect considered regards the variation on the $d_{0}$ cut for central electrons which are required to have $d_{0}<0.2$ (this is the usual cut for a no silicon hit track [6]).

For each type of systematic, reported in Table B.18 and Table B.23 the central value of the corresponding variable is the one used for the efficiency calculation, while the XX1 and XX2 indicate the corresponding varied cuts. In Table B.19, B.20, B.21, B.22, B.24, B.25 the systematic variations are reported for each parameter of the fit as the absolute differences between the values obtained using the central value and the varied one. Focusing on the plateau value of the efficiency $\left(\epsilon_{\infty}\right)$ the results in the tables point out that the main source of systematic uncertainty is the variation of the isolation cut, accounting for up to $2 \%$ of the efficiency plateau.

\begin{tabular}{|c|c|c|c|}
\hline \hline & central & XX1 & XX2 \\
\hline fake fraction & 0.05 & 0.010 & 0.1 \\
isolation $(\mathrm{GeV})$ & 4.00 & 2.00 & 8.0 \\
impact parameter $d_{0}(\mathrm{~cm})$ & 0.20 & 1000. & 0.1 \\
\hline \hline
\end{tabular}

Table B.18: CEM4 and CEM8 systematics cuts. The first column indicates the type of systematic, the second one the central value of the parameter which is to be varied and the columns labeled as XX1 and XX2 indicate the two varied values considered for the systematics study.

\begin{tabular}{|c|c|c|c|c|c|c|c|}
\hline \hline & central & fake-XX1 & fake-XX2 & iso-XX1 & iso-XX2 & $d_{0}$-XX1 & $d_{0 \text {-XX2 }}$ \\
\hline$E_{T_{0}}$ & 7.788 & -0.028495 & 0.016002 & -0.290255 & -0.038704 & -0.032709 & 0.015201 \\
$\sigma_{E_{T}}$ & 0.059 & 0.008980 & -0.011651 & 0.023955 & 0.006885 & 0.003223 & 0.010034 \\
$p_{T_{0}}$ & 7.79 & 0.068268 & -0.040489 & 0.147356 & 0.120712 & 0.036918 & 0.006044 \\
$\sigma_{p_{T}}$ & 0.0067 & -0.001886 & 0.000858 & -0.004958 & 0.000147 & 0.000443 & -0.000041 \\
$\epsilon_{\infty}$ & 0.964 & -0.004248 & -0.001191 & -0.013935 & -0.004232 & -0.000219 & 0.0033190 \\
\hline \hline
\end{tabular}

Table B.19: CEM8 systematic results for the 2-Dimensional L1 fit. The first column indicates the fit variable, the second one the central value of the variable and the other columns indicate the absolute difference between the central value and the varied one. 


\begin{tabular}{|c|c|c|c|c|c|c|c|}
\hline \hline & central & fake-XX1 & fake-XX2 & iso-XX1 & iso-XX2 & $d_{0}$-XX1 & $d_{0}$-XX2 \\
\hline$\epsilon_{\infty}$ & $9.42643 \mathrm{e}-01$ & 0.000196 & -0.000367 & 0.001434 & -0.005343 & -0.005918 & -0.000930 \\
\hline \hline
\end{tabular}

Table B.20: CEM8 systematics results for L2.

\begin{tabular}{|c|c|c|c|c|c|c|c|}
\hline \hline & central & fake-XX1 & fake-XX2 & iso-XX1 & iso-XX2 & $d_{0}$-XX1 & $d_{0}$-XX2 \\
\hline$E_{T_{0}}$ & 3.84441 & 0.000959 & -0.004460 & 0.152241 & 0.013399 & -0.003765 & -0.042324 \\
$\sigma_{E_{T}}$ & 0.063428 & -0.000958 & 0.002143 & -0.019884 & 0.003167 & -0.000953 & 0.004392 \\
$p_{T_{0}}$ & 3.377 & 0.394131 & -0.004442 & -0.069554 & 0.689487 & 0.728074 & -0.157077 \\
$\sigma_{p_{T}}$ & 0.000462 & -0.000695 & -0.001023 & -0.001326 & -0.005412 & -0.003105 & 0.000269 \\
$\epsilon_{\infty}$ & 0.89998 & 0.001184 & -0.000745 & -0.000969 & -0.021303 & -0.001309 & 0.002159 \\
\hline \hline
\end{tabular}

Table B.21: CEM4 systematics results for the 2-Dimensional L1 fit.

\begin{tabular}{|c|c|c|c|c|c|c|c|}
\hline \hline & central & fake-XX1 & fake-XX2 & iso-XX1 & iso-XX2 & $d_{0}$-XX1 & $d_{0}$-XX2 \\
\hline$\epsilon_{\infty}$ & $9.99996 \mathrm{e}-01$ & -0.000003 & 0.000022 & 0.022921 & 0.010371 & 0.000132 & -0.000003 \\
$E_{T_{0}}$ & $1.00026 \mathrm{e}-01$ & -0.000150 & -0.000063 & -0.002145 & -0.151722 & -0.000003 & -0.000060 \\
$\sigma_{E_{T}}$ & $1.41905 \mathrm{e}+00$ & -0.020906 & 0.030459 & 0.407568 & 0.135077 & 0.019400 & 0.005782 \\
\hline \hline
\end{tabular}

Table B.22: CEM4 systematics results for L2.

\begin{tabular}{|c|c|c|c|}
\hline \hline & central & XX1 & XX2 \\
\hline fake fraction & 0.15 & 0.05 & 0.25 \\
isolation & 100. & 4.00 & 8.00 \\
\hline \hline
\end{tabular}

Table B.23: PEM8 systematics cuts.

\begin{tabular}{|c|c|c|c|c|c|}
\hline \hline & central & fake-XX1 & fake-XX2 & iso-XX1 & iso-XX2 \\
\hline$E_{T_{0}}$ & 10.92 & 0.076000 & -0.130869 & 0.366624 & 0.019512 \\
$\sigma_{E_{T}}$ & 0.199 & -0.005751 & 0.002197 & 0.068721 & 0.029490 \\
$\epsilon_{\infty}$ & 0.999 & 0.000001 & -0.000000 & 0.004536 & -0.000000 \\
\hline \hline
\end{tabular}

Table B.24: PEM8 systematics results for $L 1$.

\begin{tabular}{|c|c|c|c|c|c|}
\hline \hline & central & fake-XX1 & fake-XX2 & iso-XX1 & iso-XX2 \\
\hline$\epsilon_{\infty}$ & 0.943 & 0.000362 & -0.000432 & -0.014462 & -0.016781 \\
\hline \hline
\end{tabular}

Table B.25: PEM8 systematics results for L2. 



\section{Bibliography}

[1] M.P.Giordani, S.Lammel, Dilepton Triggers for Exotic Searches at CDF II, CDF internal note 6074, Aug 2002.

[2] Refer to the web page

http ://www - cdf.fnal.gov/internal/WebTalks/Archive/

0502/050209_l2_pulsar_readiness_review/

05_050209_l2_pulsar_readiness_review_Chris_1_L2UpgMtg_020905.pdf .

[3] Private communication with Peter Wittich.

[4] S.Baroiant et Al., Measurement of Electron Trigger Efficiencies for Level1 and Level2 $8 \mathrm{GeV}$ Triggers, CDF internal note 6257, April 2003.

[5] Private communication with Carla Pilcher.

[6] S.Dube et Al., Measuring Central Electron Trigger Efficiencies for 4 GeV Triggers, CDF internal note 7095, August 2004. 



\section{List of Figures}

1.1 Chargino and neutralino associated production a p $\bar{p}$ collider. . . . . . . 8

1.2 Chargino and neutralino associated production decaying via sleptons. . . . . 9

1.3 Chargino and neutralino associated production decaying via gauge bosons. . 9

1.4 Chargino Mass Limit versus $m_{\tilde{\nu}}$ set by the LEP2. . . . . . . . . . . . . . . . 11

$1.5 \sigma \times \mathrm{BR}$ versus $\tilde{\chi}_{1}^{ \pm}$: limits set at $\mathrm{CDF}$ compared with $\mathrm{D} 0$ results during Run I. Dashed lines correspond to the theoretical prediction for four different choices of the squark masses: (a) $m_{\tilde{q}}=m_{\tilde{g}}$, (b) $m_{\tilde{q}}=1.2 m_{\tilde{g}}$, (c) $m_{\tilde{q}}=$ $1.5 m_{\tilde{g}}$, (d) $m_{\tilde{q}}=2.0 m_{\tilde{g}}$. . . . . . . . . . . . . . . . 12

1.6 Cross section for associated production of chargino and neutralino as function of $\tan \beta \ldots \ldots \ldots \ldots . \ldots \ldots 14$

$1.7 \sigma \mathrm{BR}$ versus $\tilde{\chi}_{1}^{ \pm}$: limits set at $\mathrm{D} 0$ during Run II. . . . . . . . . . . . . 14

2.1 Instantaneous luminosity peak vs.time. . . . . . . . . . . . . . . 21

2.2 Integrated luminosity vs.time. . . . . . . . . . . . . . . . . 21

2.3 Schema of the Tevatron accelerator complex. . . . . . . . . . . . 22

2.4 The CDF II detector. . . . . . . . . . . . . . . . 24

$2.5 r-\phi$ view of the CDF II detector. . . . . . . . . . . . . . . . . . . . . . . . . . . . . . . . . 25

2.6 Layout of wire planes on a COT endplate. . . . . . . . . . . . . . 26

2.7 Perspective view of IsL. . . . . . . . . . . . . . . . . . . . . . . . . . . . . . . . . . . . . . . .

$2.8 r-\phi$ view of svxiı. . . . . . . . . . . . . . . . . . . . . . . . . . . . . . . . . . . .

$2.9 r-\phi$ view of LAYER $00 . \ldots \ldots \ldots \ldots \ldots \ldots$

$2.10 r-z$ view of silicon detectors. . . . . . . . . . . . . . . . . . . . . . . . . . . . . . . . . . . . . . . . . . . . . . . .

2.11 TOF position. . . . . . . . . . . . . . . . . . . . . . . . . . . . . . . . .

2.12 Perspective view of central calorimeter half-wedge. . . . . . . . . . . 31

$2.13 r-z$ view of plug calorimeter system. . . . . . . . . . . . . . . . . . . . . . . . . . . . 32

2.14 Perspective view of plug shower maximum detector. . . . . . . . . . . . . 32

2.15 Muon system $\eta-\phi$ map. . . . . . . . . . . . . . . . . . . 34

2.16 Buffering and pipelining in the Run II CDF trigger system. . . . . . . . . . 36

3.1 Schematic diagram of CDF trigger architecture. . . . . . . . . . . 40

3.2 Schematic diagram of trigger primitives, paths and datasets. . . . . . . . 41

3.3 Graphical representation of XFT track segment finder (left) and XFT track segment linker (right). . . . . . . . . . . . . . . . . 43

3.4 Cluster finding procedure. . . . . . . . . . . . . . . . . . . . . . . . . . . . . . . . . . . 47

3.5 Tower isolation patterns. . . . . . . . . . . . . . . . . . . . . . . . . . . . . . . . . . . . .

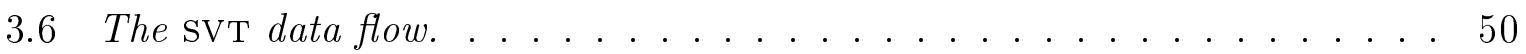

4.1 Sign convention for impact parameter. . . . . . . . . . . . . 58 
4.2 Representation of the track parameters used at CDF. . . . . . . . . 58

5.1 Chargino and neutralino associated production decaying via gauge bosons into trilepton final state. . . . . . . . . . . . . . . . 75

5.2 Production cross sections of supersymmetric processes with a center of mass energy $\sim 2 \mathrm{TeV}$ (C1N2 stands for $\chi_{1}^{ \pm} \chi_{2}^{0}$ and C1C1 C1N1 can be similarly

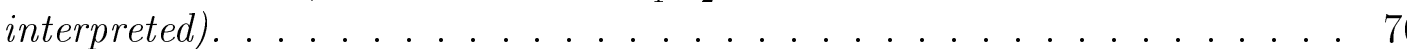

5.3 Effective production cross section of chargino and neutralino associated production in trilepton final states: $A_{0}=0, \tan \beta=5, \mu>0 . \ldots . . . .79$

5.4 Feynman diagram of a Drell-Yan event. . . . . . . . . . . . . . 80

5.5 Feynman diagram of $t \bar{t}$ production. . . . . . . . . . . . . 81

5.6 Feynman diagram of $b \bar{b}$ production. . . . . . . . . . . . . . . 81

6.1 Transverse momentum distribution for $M C$ events in which three leptons coming from the associated chargino-neutralino production are reconstructed. 89

6.2 Opposite sign dielectron distributions: the whole invariant mass spectrum. . 92

6.3 Opposite sign dielectron distributions: the $Z$ mass window. . . . . . . . . . 93

6.4 Opposite sign dimuon distributions: the whole invariant mass spectrum. . . 94

6.5 Opposite sign dimuon distributions: the $Z$ mass window. . . . . . . . . . 95

6.6 Opposite sign e $\mu$ distributions for $M C$ events after cut 1: the invariant mass and the missing energy spectra. . . . . . . . . . . . . . 96

6.7 Opposite sign e $\mu$ distributions for $M C$ events after cut 2: the invariant mass and the missing energy spectra. . . . . . . . . . . . . . . . 97

6.8 Opposite sign e $\mu$ distributions for $M C$ events after cut 3: the invariant mass and the missing energy spectra. . . . . . . . . . . . . . 97

6.9 Grafical representation of control regions. . . . . . . . . . . . . 98

6.10 Opposite sign e $\mu$ distributions: the whole invariant mass spectrum. . . . . . 100

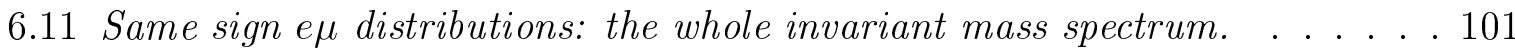

6.12 Opposite sign $\mu$ distributions: the whole invariant mass spectrum. . . . . . 102

6.13 Same sign $\mu$ distributions: the whole invariant mass spectrum. . . . . . . 103

$6.14 \mathrm{e} \mu$ distributions in control region $A+E$ : opposite sign and same sign in the top and bottom side respectively . . . . . . . . . . . . . . . . 104

$6.15 \mu$ distributions in control region $A+E$ : opposite sign and same sign in the top and bottom side respectively . . . . . . . . . . . . . . 105

$6.16 \mathrm{e} \mu$ distributions in control region $G+I$ : opposite sign and same sign in the top and bottom side respectively. . . . . . . . . . . . . . 106

$6.17 \mu$ distributions in control region $G+I$ : opposite sign and same sign in the top and bottom side respectively . . . . . . . . . . . . . . 107

$6.18 \mathrm{e} \mu$ distributions in control region A2 $+F$ : opposite sign and same sign in the top and bottom side respectively . . . . . . . . . . . . . . . 108

6.19 ue distributions in control region A2+F: opposite sign and same sign in the top and bottom side respectively. . . . . . . . . . . . . . . 109

$6.20 \mathrm{e} \mu$ distributions in control region $H+J$ : opposite sign and same sign in the top and bottom side respectively. . . . . . . . . . . . . . . . 110

6.21 ue distributions in control region $H+J$ : opposite sign and same sign in the top and bottom side respectively . . . . . . . . . . . . . . . . 111

A.1 $R$ distribution for CEM4 lepton type for each trigger path involving CEM4. 122 
A.2 $R$ distribution for CEM8 lepton type for each trigger path involving CEM8. 123

A.3 $R$ distribution for PEM8 lepton type for each trigger path involving PEM8. 124

A.4 $R$ distribution for $C M X_{4}$ lepton type for each trigger path involving $C M X_{4} .125$

A.5 $R$ distribution for $C M U_{4}$ lepton type for each trigger path involving $C M U_{4} .125$

A.6 $R$ distribution for CMUP4 lepton type for each trigger path involving CMUP4.126

A.7 Distributions of $R$ weighted average per each lepton type. . . . . . . . . . . 128

A.8 Distributions of $R$ weighted average per each lepton type after subtracting suspect runs. . . . . . . . . . . . . . . . . . . 129

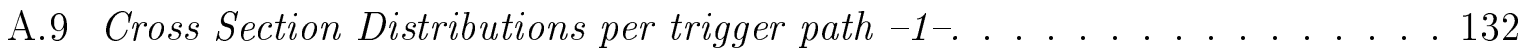

A.10 Cross Section Distributions per trigger path-2-. . . . . . . . . . . . 133

A.11 Cross Section Distributions per trigger path-3-. . . . . . . . . . . . . 134

A.12 Cross Section Distributions per trigger path -4-. . . . . . . . . . 135

B.1 $\eta$ distributions for CEM and PEM conversion candidates after ID cuts. . . 141

B.2 Distributions for CEM conversion candidates after the whole preselction. . 142

B.3 Distributions for PEM conversion candidates after the whole preselection. . 143

B.4 PEM conversion candidates distribution after ID cuts. . . . . . . . . . . 145

B.5 Distributions of matched online quantities versus the corresponding offline ones. . . . . . . . . . . . . . . . . . . 148

B.6 Distribution of L1_EM8 efficiency for the plug electron part only as a function of the offline electron $E_{T}$. The upper plot represents the efficiency of L1_EM8 as it is currently implemented in the trigger table, while the bottom plot side shows the efficiency of L1_EM8 with the $E^{H A D} / E^{E M}$ cut at $12.5 \%$, as it should be. . . . . . . . . . . . . . . . . . 149

B.7 Distribution of the difference between the offline EM cluster transverse energy and the corresponding L1 trigger quantity. . . . . . . . . . . . . . 150

B.8 Distribution of the L2_PEM8 efficiency as a function of the offline electron $E_{T}$ in $\mathrm{GeV}$ fitted with a constant. . . . . . . . . . . . . 151

B.9 Distribution of L1_CEM4_PT4 efficiency as a function of the offline elec-

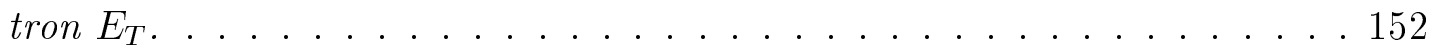

B.10 Distribution of L1_CEM4_PT4 efficiency as a function of the offline electron $p_{T} \ldots \ldots \ldots \ldots \ldots \ldots \ldots$

B.11 Distribution of L1_CEM8_PT8 efficiency as a function of the offline elec-

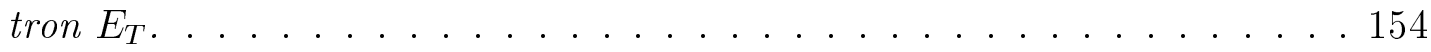

B.12 Distribution of L1_CEM8_PT8 efficiency as a function of the offline electron $p_{T}$. . . . . . . . . . . . . . . . . . . 154

B.13 Distribution of the L2_CEM4_PT4_CES2 and L2_CEM4_PT4_CES3 as a function of $E_{T} \ldots \ldots \ldots \ldots 75$

B.14 Distribution of the L2_CEM8_PT8_CES2 and L2_CEM8_PT8_CES3 as a function of $E_{T} \ldots \ldots \ldots \ldots$. . . . . . . . . . . . . . . . . . . . . . . . . . . . . 



\section{List of Tables}

1.1 The SM particles and their superpartners in the MSSM. . . . . . . . . . . 7

1.2 Standard Model prediction compared with observed data obtained at CDF Run II for the chargino-neutralino search in trileptons. The high- $p_{T} \mu e($ central) analysis requires the electron to be identified in the central region, while in the high-p $p_{T} \mu e($ forward) selection the electron must be detected in the forward calorimeter. . . . . . . . . . . . . . 15

2.1 Calorimeter $\Delta \phi \times \Delta \eta$ segmentations. . . . . . . . . . . . . . . 32

3.1 Run II bandwidths for an instataneous luminosity of $1 \times 10^{32} \mathrm{~cm}^{-2} \mathrm{~s}^{-1}$ : specifications [1] versus achieved values. . . . . . . . . . . . . . . 41

3.2 List of SUSY DILEPTON triggers before August 2003. The name of each trigger path corresponds to the "types" of leptons required. . . . . . . . . 52

3.3 SUSY DILEPTON triggers after August 2003. Since April 2004 triggers marked with $\star$ were removed because of rate restrictions, the CES threshold was raised from 2 to $3 \mathrm{GeV}$ and the L2 muon code was implemented. The last change before the 2004 shutdown took place in June 2004 when L2_CEM12_PT8 was replaced with L2_CEM8_PT8_DPS.

3.4 Standard lepton "type" definitions. The trigger primitives indicated in the middle column correspond to the trigger requirements at Level 1 and 2 for each lepton type.

4.1 Summary of resolutions on various track parameters in Run II. . . . . . . . 59

4.2 Quality assigned to vertices from the ZVertexColl algorithm. . . . . . . . 60

4.3 List of the central electron identification cuts. . . . . . . . . . . . . 68

4.4 List of the plug electron identification cuts. . . . . . . . . . . . . . 68

4.5 List of high $p_{T}$ muon identification cuts. . . . . . . . . . . . . . . . . . . . . . . . . . . . . .

4.6 List of medium $p_{T}$ muon identification cuts. . . . . . . . . . . . . . . 70

4.7 List of stubless muon identification cuts. . . . . . . . . . . . . . . . 71

5.1 Summary of the Monte Carlo samples used in the analysis. . . . . . . . . 79

6.1 Relative identification efficiencies applied to each identified lepton in $M C$ events........................ 88

6.2 Effect of the analysis cuts applied sequentially on background and signal $M C$ when selecting e $\mu$ events. . . . . . . . . . . . . 96

6.3 Number of events in signal, data and background when selecting e $\mu$ events $(O S+S S)$. 
6.4 Number of events in signal, data and background when selecting $\mu$ e events $(O S+S S)$.

6.5 Number of events in signal, data and background when selecting e $\mu+l$ events $(O S+S S) \ldots \ldots \ldots \ldots \ldots \ldots \ldots$

6.6 Number of events in signal, data and background when selecting $\mu e+l$ events $(O S+S S) \ldots \ldots \ldots \ldots \ldots$

A.1 ONLINE and OFFLINE bits required to be good for each run considered in this study. . . . . . . . . . . . . . . . . . . . 119

A.2 Standard lepton "types" are defined. The trigger primitives indicated in the middle column correspond to the trigger requirements at Level 1 and 2 for each lepton type. . . . . . . . . . . . . . . . . 120

A.3 Changes in trigger table related to steps in $R$ distributions. Starting from the left-hand side, the first column shows the trigger table change, the second column indicates which trigger paths are affected and the third column displays the first run in which the change was implemented in the trigger table. The CEM8_PEM8 path code bug was such that only one of CEM8 and PEM8 was required at trigger level. So far as CMX paths are concerned, the step in the $R$ distribution is only clearly visible for the CEM4_CMX4 trigger path. . . . . . . . . . . . . . . 121

A.4 Suspect run luminosity in $\mathrm{pb}^{-1}$ for each lepton type at STEP1 and STEP2 defined in A.3. The COT compromised period is neglected. . . . . . . . . 127

A.5 Suspect run luminosity in $p b^{-1}$ for each trigger path on the basis of cross section stability. The COT compromised period is neglected. . . . . . . . 131

B.1 Central electron identification cuts. For variable definition see Chapter 3. . 141

B.2 Forward electron identification cuts. For variable definition see Chapter 3. 141

B.3 Conversion preselection requirements. The variable $|\Delta \cot \theta| \operatorname{corresponds~to~}$ the difference in the cotangent of the polar angle between the conversion tracks while $\Delta S$ indicates their separation in the transverse $r-\phi$ plane. . 144

B.4 Estimate of the fake fraction in the CEM and PEM samples. . . . . . . . 146

B.5 Fit result for L1_EM8 efficiency as a function of $E_{T}$ as it is implemented in the trigger table. . . . . . . . . . . . . . . . 150

B.6 Fit result for L1_EM8 efficiency as a function of $E_{T}$ with the $E^{H A D} / E^{E M}$ cut at $12.5 \%$. . . . . . . . . . . . . . . 150

B.7 Fit result for L2_PEM8 efficiency as a constant function of $E_{T}$. . . . . . . 151

B.8 Fit result for L1_CEM4_PT4 efficiency as a function of $E_{T}$. . . . . . . . . 152

B.9 Fit result for L1_CEM4_PT4 efficiency as a constant function of $p_{T}$. . . . . 152

B.10 Fit result for L1_CEM8_PT8 efficiency as a function of $E_{T}$. . . . . . . . . 155

B.11 Fit result for L1_CEM8_PT8 efficiency as a function of $p_{T}$. . . . . . . . . 155

B.12 2-Dimensional fit result for L1_CEM4_PT4. . . . . . . . . . . . . . . 155

B.13 2-Dimensional fit result for L1_CEM8_PT8. . . . . . . . . . . . . . 155

B.14 Fit result for L2_CEM4_PT4_CES2. . . . . . . . . . . . . . . . . 156

B.15 Fit result for L2_CEM4_PT4_CES3. . . . . . . . . . . . . . . . . . 156

B.16 Fit result for L2_CEM8_PT8_CES2. . . . . . . . . . . . . . . . 158

B.17 Fit result for L2_CEM8_PT8_CES3. . . . . . . . . . . . . . . . . 158 
B.18 CEM4 and CEM8 systematics cuts. The first column indicates the type of systematic, the second one the central value of the parameter which is to be varied and the columns labeled as XX1 and XX2 indicate the two varied values considered for the systematics study. . . . . . . . . . . . . . . 160

B.19 CEM8 systematic results for the 2-Dimensional L1 fit. The first column indicates the fit variable, the second one the central value of the variable and the other columns indicate the absolute difference between the central value and the varied one. . . . . . . . . . . . . . . . 160

B.20 CEM8 systematics results for L2. . . . . . . . . . . . . . . . 161

B.21 CEM4 systematics results for the 2-Dimensional L1 fit. . . . . . . . . . . . 161

B.22 CEM4 systematics results for L2. . . . . . . . . . . . . . . . . . . 161

B.23 PEM8 systematics cuts. . . . . . . . . . . . . . . . . . . . . . . . . . . . . . . . . .

B.24 PEM8 systematics results for L1. . . . . . . . . . . . . . . . . 161

B.25 PEM8 systematics results for L2. . . . . . . . . . . . . . . . . . . 161 